\title{
Nickel-Catalyzed Reductive Arylalkylation via a Migratory Insertion/Decarboxylative Cross-Coupling Cascade
}

\author{
Youxiang Jin ${ }^{\mathrm{a}}$, Haobo Yang ${ }^{\mathrm{b}}$ and Chuan Wang ${ }^{\mathrm{a}}{ }^{\mathrm{k}}$
}

\begin{abstract}
${ }^{a}$ Hefei National Laboratory for Physical Science at the Microscale, Department of Chemistry, University of Science and Technology of China, Center for Excellence in Molecular Synthesis, Hefei, Anhui, 230026 (China). E-mail: chuanw@ustc.edu.cn

${ }^{b}$ School of Chemistry and Chemical Engineering, Shanghai Jiao Tong University, 800 Dongchuan Road, Shanghai, 200240 (P. R. China).
\end{abstract}

\section{Table of Contents}

General Methods and Materials. 2

Procedures for the Synthesis of Alkenes with Tethered Aryl Iodides ....................................................................... 3

General Procedure for the Ni-Catalyzed Reductive Arylalkylation of Unactivated Olefins ................................................ 9

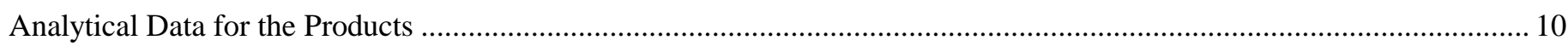

Control Experiments for the Ni-catalyzed Reductive Arylalkylation Involving Decarboxylative Coupling ........................ 19

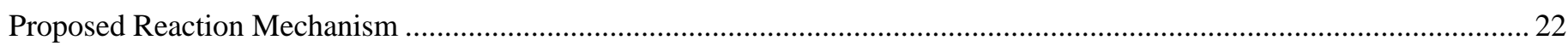

Asymmetric Ni-catalyzed Reductive Arylalkylation involving Decarboxylative Coupling ............................................. 23

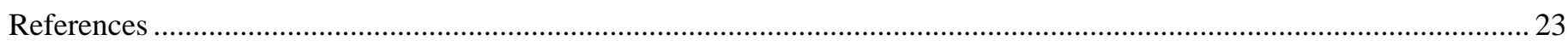

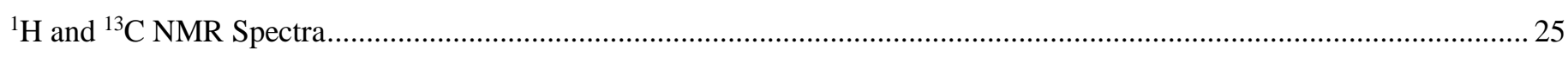

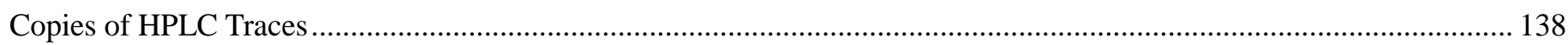




\section{General Methods and Materials}

${ }^{1} \mathrm{H}$ NMR and ${ }^{13} \mathrm{C}$ NMR spectra were recorded on a Bruker Advance 400M NMR spectrometers at ambient temperature in $\mathrm{CDCl}_{3}$ at 400 and $101 \mathrm{MHz}$. The chemical shifts are given in ppm relative to tetramethylsilane $\left[{ }^{1} \mathrm{H}: \delta=\left(\mathrm{SiMe}_{4}\right)=0.00 \mathrm{ppm}\right]$ as an internal standard or relative to the resonance of the solvent $\left[{ }^{1} \mathrm{H}: \delta=\left(\mathrm{CDCl}_{3}\right)=7.26,{ }^{13} \mathrm{C}: \delta=\left(\mathrm{CDCl}_{3}\right)=77.16 \mathrm{ppm}\right]$. Multiplicities were given as: s (singlet); $\mathrm{d}$ (doublet); $\mathrm{t}$ (triplet); $\mathrm{q}$ (quartet); dd (doublet of doublets); dt (doublet of triplets); m (multiplets), etc. Coupling constants are reported as $J$ values in Hz. High resolution mass spectral analysis (HRMS) was performed on Waters XEVO G2 Q-TOF. HPLC was performed on Thermo UltiMate 3000. Flash chromatography was performed using 200-300 mesh silica gel with the indicated solvent system.

Alkenes 1a, ${ }^{1} \mathbf{1 k},{ }^{1} \mathbf{1},{ }^{1} \mathbf{1 m},{ }^{2} \mathbf{1 n},{ }^{1} \mathbf{1} \mathbf{p},{ }^{3} \mathbf{1 q},{ }^{4} \mathbf{1} \mathbf{r},{ }^{4} \mathbf{1} \mathbf{u},{ }^{1} \mathbf{1} \mathbf{v}^{1}, \mathbf{1} \mathbf{x}^{5}, \mathbf{1} \mathbf{y}^{6}$, NHP Esters $\mathbf{2},{ }^{7}$ and Ligands $\mathbf{L} \mathbf{1}^{8}, \mathbf{L 3}^{8}$ and $\mathbf{L 5}^{8}$ were prepared according to the procedures reported in the literature. All other reagents and starting materials, unless otherwise noted, were purchased from commercial vendors and used without further purification. 
Procedures for the Synthesis of Alkenes with Tethered Aryl Iodides<smiles>C=C(C)CCc1ccccc1I</smiles>

$1 \mathbf{a}$<smiles>C=C(CC)CCc1ccccc1I</smiles>

$1 \mathrm{~b}$<smiles>C=C(CCc1ccccc1I)NPCC</smiles>

$1 \mathrm{c}$

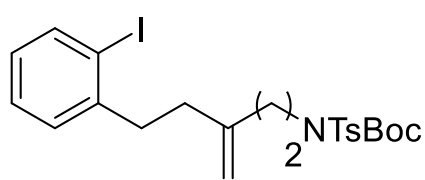

$1 d$<smiles>C=C(CCc1ccccc1I)NC(C)=O</smiles>

$1 e$

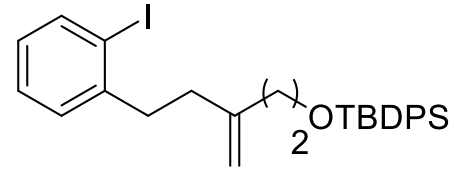

$1 \mathrm{~h}$<smiles>C=C(C)CCc1cc2c(cc1I)OCO2</smiles>

11<smiles>C=C(C)COCc1ccccc1I</smiles>

$1 p$<smiles>C=C(C)COc1ccccc1I</smiles>

$1 \mathrm{~m}$<smiles>C=C(C)CN(C)c1ccccc1I</smiles>

$1 q$<smiles>C=C(C)COc1ccc(C(C)(C)C)cc1I</smiles>

1n<smiles>C=C(C)CN(CC(=C)C)c1ccccc1I</smiles><smiles>C=C(C)CCCCOc1ccccc1I</smiles>
$1 v$<smiles>CC(=CC1CC1)CCc1ccccc1I</smiles><smiles>C=C(C)COc1ccc(C#CCCC)cc1I</smiles>

10<smiles>C=C([M]O)CN(Cc1ccccc1I)Cc1ccccc1I</smiles>
$1 \mathrm{~s}$ $1 k$<smiles>C=C(C)CN([As])Cc1ccccc1I</smiles>

$1 t$<smiles>C/C=C\CCc1ccccc1I</smiles>

$1 x$<smiles>C=C(C)CCCOc1ccccc1I</smiles>

$1 \mathrm{u}$<smiles>C=CCCc1ccccc1I</smiles>

$1 y$ 

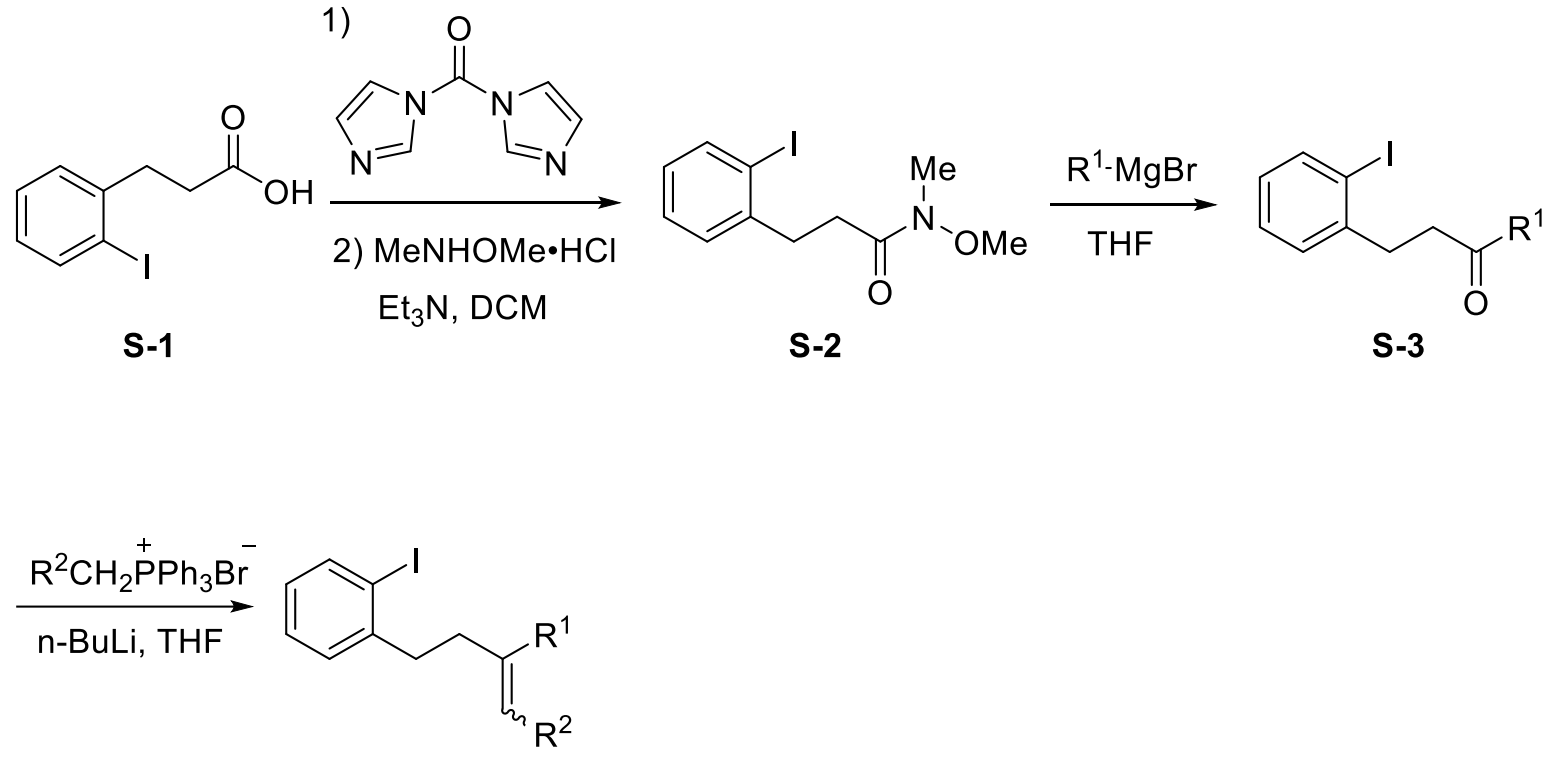

$1 \mathrm{~b}, 1 \mathrm{c}, 1 \mathrm{i}, 1 \mathrm{j}$ and $1 \mathrm{w}$

Step 1: To a $250 \mathrm{~mL}$ round-bottomed flask equipped with a stir bar was added 3-(2-iodophenyl)propanoic acid $\mathbf{S}-\mathbf{1}^{9}$ (2.22 g, $8.1 \mathrm{mmol}, 1.0$ equiv) and $\mathrm{CH}_{2} \mathrm{Cl}_{2}(100 \mathrm{~mL})$. To this stirred solution was added 1,1'- carbonyldiimidazole $(1.57 \mathrm{~g}, 9.7 \mathrm{mmol}$, 1.2 equiv) in one portion, turning the solution a clear pale-yellow and resulting in the evolution of $\mathrm{CO}_{2}$ gas. The reaction mixture was allowed to stir for $1 \mathrm{~h}$ at r.t. After this time, $\mathrm{N}$-O-dimethylhydroxylamine hydrochloride $(0.95 \mathrm{~g}, 9.7 \mathrm{~mol}, 1.2$ equiv) and $\mathrm{Et}_{3} \mathrm{~N}(1.79 \mathrm{~g}, 2.50 \mathrm{~mL}, 17.8 \mathrm{mmol}, 2.5$ equiv) were added all at once, and the reaction mixture was stirred overnight, before it was then quenched with $50 \mathrm{~mL}$ of $1 \mathrm{M}$ aq. $\mathrm{HCl}$ and stirred vigorously for $10 \mathrm{~min}$. Next, the solution was transferred to a separatory funnel, and the layers were separated. The aq. layer was extracted with $\mathrm{CH}_{2} \mathrm{Cl}_{2}(3 \times 30 \mathrm{~mL})$. The combined organic layers were washed with $1 \mathrm{M}$ aq. $\mathrm{HCl}(50 \mathrm{~mL})$, deionized $\mathrm{H}_{2} \mathrm{O}(50 \mathrm{~mL})$, saturated aq. $\mathrm{NaHCO}_{3}(2 \times 50 \mathrm{~mL})$, and brine $(50 \mathrm{~mL})$, dried over anhydrous $\mathrm{Na}_{2} \mathrm{SO}_{4}$, filtered and removed in vacuo via rotary evaporation. The residue was purified through column chromatography on silica gel (petroleum ether:EtOAc=10:1), affording the pure product 3-(2-iodophenyl)$N$-methoxy-N-methylpropanamide S-2 (2.43 g, $94 \%)$ as a clear, light yellow oil.

${ }^{1} \mathrm{H}$ NMR (400 MHz, $\left.\mathrm{CDCl}_{3}\right) \delta=7.84-7.78(\mathrm{~m}, 1 \mathrm{H}), 7.31-7.24(\mathrm{~m}, 2 \mathrm{H}), 6.93-6.86(\mathrm{~m}, 1 \mathrm{H}), 3.63(\mathrm{~s}, 3 \mathrm{H}), 3.18(\mathrm{~s}, 3 \mathrm{H}), 3.09-$ $3.03(\mathrm{~m}, 2 \mathrm{H}), 2.74(\mathrm{t}, J=8.0 \mathrm{~Hz}, 2 \mathrm{H}) \mathrm{ppm} ;{ }^{13} \mathrm{C} \mathrm{NMR}\left(101 \mathrm{MHz}, \mathrm{CDCl}_{3}\right) \delta=143.9,139.5(2 \mathrm{C}), 129.9,128.5,128.1,100.3$, $61.3,35.7(2 \mathrm{C}), 32.3 \mathrm{ppm}$.

Step 2: A solution of $\mathrm{RMgBr}(7 \mathrm{mmol}, 2.0$ equiv) in dry tetrahydrofuran $(30 \mathrm{~mL})$ was added dropwise to a mixture of 3-(2iodophenyl)- $N$-methoxy- $N$-methylpropanamide $\mathbf{S - 2}\left(1.12 \mathrm{~g}, 3.5 \mathrm{mmol}, 1.0\right.$ equiv) under nitrogen at $0{ }^{\circ} \mathrm{C}$. The mixture was stirred at room temperature for $2 \mathrm{~h}$, which was then quenched with water and the aqueous phase was extracted with ethyl acetate. The combined organic layers were washed with brine, dried over anhydrous sodium sulfate, filtered and concentrated to give the crude ketones $\mathbf{S - 3}$, which was used in the next step without further purification.

Step 3: To a dry tetrahydrofuran $(15 \mathrm{~mL})$ solution of alkyltriphenylphosphonium bromide ( $1.57 \mathrm{~g}, 4.4 \mathrm{mmol}, 1.25$ equiv) was added $n$-butyl lithium $\left(2.5 \mathrm{M}\right.$ in hexane, $1.5 \mathrm{~mL}, 3.85 \mathrm{mmol}, 1.1$ equiv) at $0{ }^{\circ} \mathrm{C}$. After the mixture was stirred at room temperature for $30 \mathrm{~min}$, a tetrahydrofuran solution of the crude ketones $\mathbf{S - 3}\left(3.5 \mathrm{mmol}, 1.0\right.$ equiv) was added dropwise at $0^{\circ} \mathrm{C}$. After stirring at room temperature for $14 \mathrm{~h}$, the mixture was quenched with water and the aqueous layer was extracted with ethyl acetate. The combined organic phases were washed with brine, dried over anhydrous $\mathrm{Na}_{2} \mathrm{SO}_{4}$, filtered and concentrated in vacuo. The residue was purified by column chromatography on silica gel with petroleum ether to give the alkenes $\mathbf{1 b}, \mathbf{1 c}$, 
<smiles>C=C(CC)CCc1ccccc1I</smiles><smiles>C=C(CCc1ccccc1I)NCC</smiles><smiles>CC=C(C)CCc1ccccc1I</smiles>
$1 i$

1-Iodo-2-(3-methylenepentyl)benzene (1b) was isolated as a colorless oil (0.80 g, $2.80 \mathrm{mmol}, 80 \%$ yield). ${ }^{1} \mathrm{H} \mathrm{NMR}\left(400 \mathrm{MHz}, \mathrm{CDCl}_{3}\right) \delta=7.81(\mathrm{dd}, J=7.9,1.2 \mathrm{~Hz}, 1 \mathrm{H}), 7.28-7.19(\mathrm{~m}, 2 \mathrm{H}), 6.87(\mathrm{td}, J=$ $7.5,1.9 \mathrm{~Hz}, 1 \mathrm{H}), 4.82-4.75(\mathrm{~m}, 2 \mathrm{H}), 2.88-2.79(\mathrm{~m}, 2 \mathrm{H}), 2.33-2.24(\mathrm{~m}, 2 \mathrm{H}), 2.12(\mathrm{q}, J=7.5 \mathrm{~Hz}, 2 \mathrm{H})$, $1.07(\mathrm{t}, J=7.4 \mathrm{~Hz}, 3 \mathrm{H}) \mathrm{ppm} ;{ }^{13} \mathrm{C} \mathrm{NMR}\left(101 \mathrm{MHz}, \mathrm{CDCl}_{3}\right) \delta=150.6,144.8,139.5,129.3,128.4,127.7$, $108.3,100.5,39.7,36.8,29.0,12.5 \mathrm{ppm}$.

1-Iodo-2-(3-methylenehexyl)benzene (1c) was isolated as a colorless oil (0.79 g, $2.63 \mathrm{mmol}, 75 \%$ yield). ${ }^{1} \mathrm{H}$ NMR $\left(400 \mathrm{MHz}, \mathrm{CDCl}_{3}\right) \delta=7.81(\mathrm{dd}, J=7.9,1.2 \mathrm{~Hz}, 1 \mathrm{H}), 7.29-7.18(\mathrm{~m}, 2 \mathrm{H}), 6.87(\mathrm{td}, J$ $=7.5,1.9 \mathrm{~Hz}, 1 \mathrm{H}), 4.85-4.72(\mathrm{~m}, 2 \mathrm{H}), 2.91-2.77(\mathrm{~m}, 2 \mathrm{H}), 2.32-2.21(\mathrm{~m}, 2 \mathrm{H}), 2.07(\mathrm{t}, J=7.6 \mathrm{~Hz}, 3 \mathrm{H})$, $1.57-1.43(\mathrm{~m}, 2 \mathrm{H}), 0.92(\mathrm{t}, J=7.3 \mathrm{~Hz}, 3 \mathrm{H}) \mathrm{ppm} ;{ }^{13} \mathrm{C} \mathrm{NMR}\left(101 \mathrm{MHz}, \mathrm{CDCl}_{3}\right) \delta=148.9,144.8,139.5$, $129.3,128.3,127.7,109.5,100.5,39.7,38.4,36.5,21.0,13.9$ ppm.

1-Iodo-2-(3-methylpent-3-en-1-yl)benzene (1i) was isolated as a colorless oil (0.66 g, $2.31 \mathrm{mmol}, 66 \%$ yield). ${ }^{1} \mathrm{H}$ NMR $\left(400 \mathrm{MHz}, \mathrm{CDCl}_{3}\right) \delta=7.84-7.76(\mathrm{~m}, 1 \mathrm{H}), 7.29-7.23(\mathrm{~m}, 1 \mathrm{H}), 7.22-7.17(\mathrm{~m}, 1 \mathrm{H}), 6.90-$ $6.83(\mathrm{~m}, 1 \mathrm{H}), 5.33-5.20(\mathrm{~m}, 1 \mathrm{H}), 2.86-2.71(\mathrm{~m}, 2 \mathrm{H}), 2.33-2.26(\mathrm{~m}, 1 \mathrm{H})[2.25-2.19(\mathrm{~m}, 1 \mathrm{H})], 1.79-1.74$ $(\mathrm{m}, 1.5 \mathrm{H})[1.72-1.68(\mathrm{~m}, 1.5 \mathrm{H})], 1.59(\mathrm{~d}, J=6.7 \mathrm{~Hz}, 1.5 \mathrm{H})[1.51(\mathrm{~d}, J=6.7 \mathrm{~Hz}, 1.5 \mathrm{H})] \mathrm{ppm} ;{ }^{13} \mathrm{C} \mathrm{NMR}$ $\left(101 \mathrm{MHz}, \mathrm{CDCl}_{3}\right) \delta=145.0$ (144.8), 139.42, 134.9 (134.7), 129.5 (129.4), 128.3, 127.7 (127.6), 120.2 (119.2), 100.5 (100.4), 40.3 (39.9), 39.1 (32.28), 23.5 (15.87), 13.4 (13.3) ppm. Inside the brackets are the chemical shifts of another diastereomer.<smiles>CC=CC(C)=CCc1ccccc1I</smiles>

1j

1-Iodo-2-(3-methylhex-3-en-1-yl)benzene $(\mathbf{1 j})$ was isolated as a colorless oil $(0.56 \mathrm{~g}, 1.86 \mathrm{mmol}, 53 \%$ yield). ${ }^{1} \mathrm{H}$ NMR $\left(400 \mathrm{MHz}, \mathrm{CDCl}_{3}\right) \delta=7.85-7.75(\mathrm{~m}, 1 \mathrm{H}), 7.30-7.15(\mathrm{~m}, 2 \mathrm{H}), 6.92-6.82(\mathrm{~m}, 1 \mathrm{H}), 5.22-$ $5.12(\mathrm{~m}, 1 \mathrm{H}), 2.84-2.72(\mathrm{~m}, 2 \mathrm{H}), 2.32-2.23(\mathrm{~m}, 1 \mathrm{H})$ [2.26-2.17 (m, 1H)], 2.05-1.97 (m, 1H) [1.97-1.90 $(\mathrm{m}, 1 \mathrm{H})], 1.80-1.74(\mathrm{~m}, 1.5 \mathrm{H})[1.72-1.65(\mathrm{~m}, 1.5 \mathrm{H})], 0.93(\mathrm{t}, J=7.5 \mathrm{~Hz}, 1.5 \mathrm{H})[0.87(\mathrm{t}, J=7.5 \mathrm{~Hz}$, 1.5H)] ppm; ${ }^{13} \mathrm{C}$ NMR (101 MHz, $\left.\mathrm{CDCl}_{3}\right) \delta=139.7$ (139.6), 134.22 (134.18), 128.2 (127.9), 124.3 (124.2), 123.2 (123.1), 123.0 (122.5), 122.4 (122.1), 95.3 (95.1), 35.0 (34.7), 34.3 (27.4), 18.2 (16.0), 15.9 (10.8), 9.4 (9.1) ppm. Inside the brackets are the chemical shifts of another diastereomer.

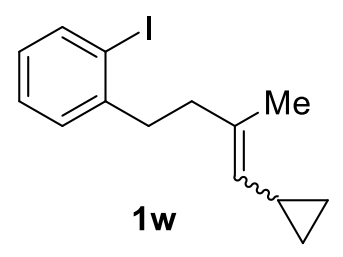

1-(4-cyclopropyl-3-methylbut-3-en-1-yl)-2-iodobenzene (1w) was isolated as a colorless oil $(0.44 \mathrm{~g}$, $1.40 \mathrm{mmol}, 40 \%$ yield). ${ }^{1} \mathrm{H}$ NMR $\left(400 \mathrm{MHz}, \mathrm{CDCl}_{3}\right) \delta=7.84-7.76(\mathrm{~m}, 1 \mathrm{H}), 7.29-7.15(\mathrm{~m}, 2 \mathrm{H}), 6.91-$ $6.82(\mathrm{~m}, 1 \mathrm{H}), 4.62-4.54(\mathrm{~m}, 1 \mathrm{H}), 2.88-2.75(\mathrm{~m}, 2 \mathrm{H}), 2.44-2.38(\mathrm{~m}, 1 \mathrm{H})[2.25-2.16(\mathrm{~m}, 1 \mathrm{H})], 1.85-$ $1.82(\mathrm{~m}, 1.5 \mathrm{H}), 1.77-1.74(\mathrm{~m}, 1.5 \mathrm{H}), 1.51-1.39(\mathrm{~m}, 1 \mathrm{H}), 0.73-0.66(\mathrm{~m}, 1 \mathrm{H})[0.65-0.58(\mathrm{~m}, 1 \mathrm{H})], 0.30-$ $0.24(\mathrm{~m}, 1 \mathrm{H})[0.24-0.18(\mathrm{~m}, 1 \mathrm{H})] \mathrm{ppm} ;{ }^{13} \mathrm{C} \mathrm{NMR}\left(101 \mathrm{MHz}, \mathrm{CDCl}_{3}\right) \delta=144.9$ (144.8), 139.4, 133.1 (132.9), 130.3 (129.6), 129.4 (129.3), 128.28 (128.26), 127.7 (127.6), 100.5 (100.4), 40.1, 39.9 (39.4), 33.1, 23.4 (16.6), 10.02 (9.96), 6.65 (6.62) ppm. Inside the brackets are the chemical shifts of another diastereomer. 
Procedure for Synthesis of 5-(2-Iodophenyl)-3-methylenepentan-1-ol (S-4) ${ }^{10}$<smiles>C=C(C)CCO</smiles>

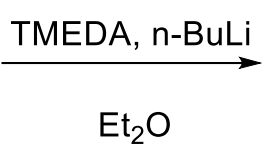<smiles>C=C(CCO)CCc1ccccc1I</smiles>

S-4

To an ice-cooled solution of tetramethylethylenediamine ( $8 \mathrm{~mL}, 53 \mathrm{mmol}, 5.3$ equiv) in $\mathrm{Et}_{2} \mathrm{O}$ ( $30 \mathrm{~mL}$ ) was added $n$ - $\mathrm{BuLi}(2.5$ $\mathrm{M}$ in hexanes, $16 \mathrm{~mL}, 40 \mathrm{mmol}, 4$ equiv). After stirring at room temperature for $1 \mathrm{~h}$, the solution was then cooled to $0{ }^{\circ} \mathrm{C}$, and 3-methyl-3-buten-1-ol ( $2 \mathrm{~mL}, 20 \mathrm{mmol}, 2$ equiv) and 1-(bromomethyl)-2-iodobenzene (2.96 g, $10 \mathrm{mmol}, 1$ equiv) in $\mathrm{Et}_{2} \mathrm{O}$ (5 $\mathrm{mL}$ ) were added dropwise, successively. The reaction mixture was allowed to warm up to room temperature and stirred for 6 $h$, before it was quenched with saturated aq. $\mathrm{NH}_{4} \mathrm{Cl}$ and diluted with $\mathrm{H}_{2} \mathrm{O}$. The aqueous phase was extracted three times by $\mathrm{Et}_{2} \mathrm{O}$ and the combined organic layers was washed successively with $1 \mathrm{M}$ aq. $\mathrm{HCl}$ and brine, dried over anhydrous $\mathrm{Na}_{2} \mathrm{SO}_{4}$, filtered and concentrated in vacuo. Purification by column chromatography (petroleum ether/ethyl acetate $=4: 1$ to $2: 1$ ) on silica gel gave the pure product $\mathbf{S}-4$ as a yellow oil $\left(1.51 \mathrm{~g}, 5.0 \mathrm{mmol}, 50 \%\right.$ yield). ${ }^{1} \mathrm{H} \mathrm{NMR}\left(400 \mathrm{MHz}, \mathrm{CDCl}_{3}\right) \delta=7.81$ (dd, $J=7.9,1.3 \mathrm{~Hz}, 1 \mathrm{H}), 7.27(\mathrm{td}, J=7.4,1.3 \mathrm{~Hz}, 1 \mathrm{H}), 7.22(\mathrm{dd}, J=7.6,1.9 \mathrm{~Hz}, 1 \mathrm{H}), 6.89(\mathrm{td}, J=7.5,1.9 \mathrm{~Hz}, 1 \mathrm{H}), 5.01-4.87(\mathrm{~m}$, $2 \mathrm{H}), 3.77(\mathrm{t}, J=6.3 \mathrm{~Hz}, 2 \mathrm{H}), 2.90-2.81(\mathrm{~m}, 2 \mathrm{H}), 2.38(\mathrm{t}, J=6.3 \mathrm{~Hz}, 2 \mathrm{H}), 2.35-2.26(\mathrm{~m}, 2 \mathrm{H}) \mathrm{ppm} ;{ }^{13} \mathrm{C} \mathrm{NMR}\left(101 \mathrm{MHz}, \mathrm{CDCl}_{3}\right)$ $\delta=145.1,144.3,139.5,129.4,128.4,127.9,112.4,100.4,60.4,39.5,39.3,36.3 \mathrm{ppm}$.

\section{Procedure for Synthesis of $N$-(5-(2-Bromophenyl)-3-methylenepentyl)-4-methylbenzenesulfonamide (1d)}<smiles>C=C(CCO)CCc1ccccc1I</smiles>

S-4

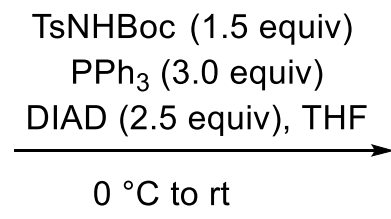

$0^{\circ} \mathrm{C}$ to $\mathrm{rt}$

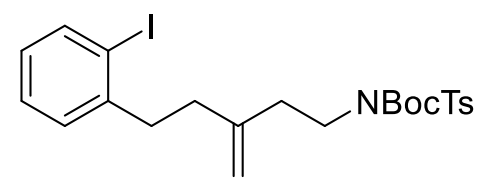

$1 d$

To a stirred solution of tert-butyl tosylcarbamate $(2.03 \mathrm{~g}, 7.5 \mathrm{mmol}, 1.5$ equiv), the homoallylic alcohol $\mathbf{S}-4(1.51 \mathrm{~g}, 5.0 \mathrm{mmol}$, 1.0 equiv), and triphenylphosphine ( $3.93 \mathrm{~g}, 15.0 \mathrm{mmol}, 3$ equiv) in THF ( $25 \mathrm{~mL})$, DIAD ( $2.53 \mathrm{~g}, 12.5 \mathrm{mmol}, 2.5$ equiv) was added at $0{ }^{\circ} \mathrm{C}$. After stirring at room temperature overnight, the mixture was quenched with water and the aqueous phase was extracted with ethyl acetate. The combined organic layers were washed with brine, dried over anhydrous $\mathrm{Na}_{2} \mathrm{SO}_{4}$, filtered and concentrated. The residue was purified by column chromatography (petroleum ether/ethyl acetate $=5: 1$ ) on silica gel to give 1d as a yellow oil (2.08 g, $3.75 \mathrm{mmol}, 75 \%$ yield). ${ }^{1} \mathrm{H}$ NMR (400 MHz, $\left.\mathrm{CDCl}_{3}\right) \delta=7.83-7.75(\mathrm{~m}, 2 \mathrm{H}), 7.34-7.21(\mathrm{~m}, 5 \mathrm{H})$, 6.93-6.84 (m, 1H), 4.97-4.83 (m, 2H), 4.02-3.88 (m, 2H), 2.92-2.83 (m, 2H), 2.59-2.50 (m, 2H), $2.44(\mathrm{~s}, 3 \mathrm{H}), 2.38-2.31(\mathrm{~m}$, 2H), 1.34 (s, 9H) ppm; ${ }^{13} \mathrm{C}$ NMR (101 MHz, $\left.\mathrm{CDCl}_{3}\right) \delta=150.9,145.2,144.4,144.1,139.4,137.5,129.5,129.3$ (2C), 128.4, 127.9 (2C), 127.8, 112.3, 100.5, 84.2, 46.2, 39.5, 36.7, 36.5, 27.9 (3C), $21.7 \mathrm{ppm}$.

\section{Procedure for Synthesis of Alkenes 1e and if}<smiles>C=C(CCO)CCc1ccccc1I</smiles>

S-4<smiles>[R]C(=O)OCCC(=C)CCc1ccccc1I</smiles>

1e, $1 f$

To a stirred solution of $\mathbf{S}-4\left(1.51 \mathrm{~g}, 5.0 \mathrm{mmol}, 1.0\right.$ equiv) and $\mathrm{Et}_{3} \mathrm{~N}\left(0.80 \mathrm{~mL}, 5.5 \mathrm{mmol}, 1.1\right.$ equiv) in $\mathrm{Et}_{2} \mathrm{O}(10 \mathrm{~mL})$ were 
added acyl chlorides $\left(5.0 \mathrm{mmol}, 1.0\right.$ equiv) at $0{ }^{\circ} \mathrm{C}$. After stirring at this temperature for $1 \mathrm{~h}$ and then at room temperature for $8.5 \mathrm{~h}$, the reaction mixture was quenched with water and the aqueous phase was extracted twice with EtOAc. The combined organic layers were dried over anhydrous $\mathrm{Na}_{2} \mathrm{SO}_{4}$, filtered, and evaporated to dryness. The crude residue was purified by column chromatography (petroleum ether/ethyl acetate $=20: 1$ ) on silica gel to give the products $\mathbf{1 e}$ and $\mathbf{1 f}$.

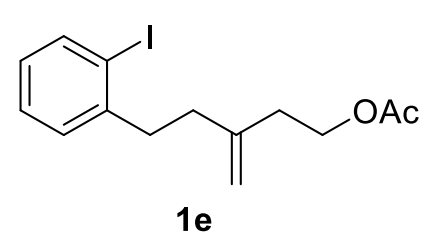

5-(2-Iodophenyl)-3-methylenepentyl acetate (1e) was isolated as a yellow oil $(1.32 \mathrm{~g}, 3.85$ mmol, $77 \%$ yield). ${ }^{1} \mathrm{H}$ NMR $\left(400 \mathrm{MHz}, \mathrm{CDCl}_{3}\right) \delta=7.81$ (dd, $\left.J=7.9,1.3 \mathrm{~Hz}, 1 \mathrm{H}\right), 7.27(\mathrm{td}, J$ $=7.4,1.3 \mathrm{~Hz}, 1 \mathrm{H}), 7.21(\mathrm{dd}, J=7.6,1.9 \mathrm{~Hz}, 1 \mathrm{H}), 6.89(\mathrm{td}, J=7.5,1.9 \mathrm{~Hz}, 1 \mathrm{H}), 4.95-4.82(\mathrm{~m}$, $2 \mathrm{H}), 4.23(\mathrm{t}, J=7.0 \mathrm{~Hz}, 2 \mathrm{H}), 2.90-2.78(\mathrm{~m}, 2 \mathrm{H}), 2.43(\mathrm{t}, J=6.9 \mathrm{~Hz}, 3 \mathrm{H}), 2.35-2.26(\mathrm{~m}, 2 \mathrm{H})$, 2.06 (s, 3H) ppm; ${ }^{13} \mathrm{C} \mathrm{NMR}\left(101 \mathrm{MHz}, \mathrm{CDCl}_{3}\right) \delta=171.1,144.6,144.3,139.5,129.4,128.4$,

$127.9,111.9,100.4,62.9,39.5,36.7,35.1,21.1 \mathrm{ppm}$.

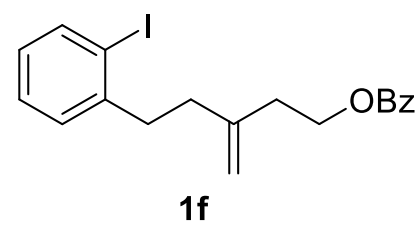

5-(2-Iodophenyl)-3-methylenepentyl benzoate (1f) was isolated as a yellow oil (1.68 g, 4.15 mmol, $83 \%$ yield). ${ }^{1} \mathrm{H}$ NMR (400 MHz, $\left.\mathrm{CDCl}_{3}\right) \delta=8.09-8.00(\mathrm{~m}, 2 \mathrm{H}), 7.81$ (dd, $J=7.9,1.2$ $\mathrm{Hz}, 1 \mathrm{H}), 7.60-7.51(\mathrm{~m}, 1 \mathrm{H}), 7.47-7.38(\mathrm{~m}, 2 \mathrm{H}), 7.30-7.17(\mathrm{~m}, 2 \mathrm{H}), 6.93-6.83(\mathrm{~m}, 1 \mathrm{H}), 4.99-$ $4.90(\mathrm{~m}, 2 \mathrm{H}), 4.48(\mathrm{t}, J=6.8 \mathrm{~Hz}, 2 \mathrm{H}), 2.93-2.82(\mathrm{~m}, 2 \mathrm{H}), 2.57(\mathrm{t}, J=6.8 \mathrm{~Hz}, 2 \mathrm{H}), 2.41-2.31$ $(\mathrm{m}, 2 \mathrm{H}) \mathrm{ppm} ;{ }^{13} \mathrm{C} \mathrm{NMR}\left(101 \mathrm{MHz}, \mathrm{CDCl}_{3}\right) \delta=166.6,144.6,144.3,139.5,132.9,130.35,129.6$ (2C), 129.4, 128.40, 128.36 (2C), 127.9, 112.2, 100.5, 63.4, 39.5, 36.6, $35.2 \mathrm{ppm}$.

\section{Procedure for Synthesis of 5-(2-Bromophenyl)-3-methylenepentyl furan-2-carboxylate (1g)}
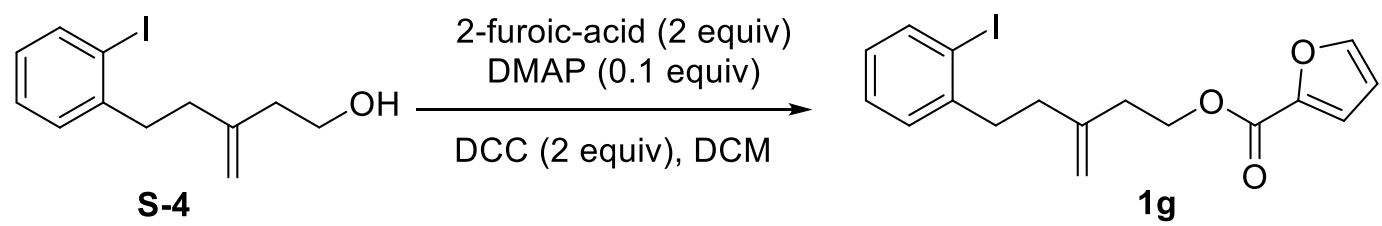

To a solution of the homoallylic alcohol S-4 (1.51 g, $5.0 \mathrm{mmol}, 1.0$ equiv), 2-furoic acid (1.1 g, $10.0 \mathrm{mmol}, 2.0$ equiv) and DMAP (61 mg, $0.5 \mathrm{mmol}, 10 \mathrm{~mol} \%)$ in $\mathrm{CH}_{2} \mathrm{Cl}_{2}\left(35 \mathrm{~mL}\right.$ ) was added a solution of DCC (2.0 g, $10.0 \mathrm{mmol}, 2.0$ equiv) in $\mathrm{CH}_{2} \mathrm{Cl}_{2}$ $(15 \mathrm{~mL})$. After stirring at room temperature for $12 \mathrm{~h}$, the reaction mixture was filtered through a pad of silica gel. The filtrate was concentrated, and the crude residue was purified by column chromatography (petroleum ether/ethyl acetate $=10: 1$ ) on silica gel to give $1 \mathrm{~g}$ as a yellow oil $\left(1.50 \mathrm{~g}, 3.80 \mathrm{mmol}, 76 \%\right.$ yield). ${ }^{1} \mathrm{H}$ NMR $\left(400 \mathrm{MHz}, \mathrm{CDCl}_{3}\right) \delta=7.81(\mathrm{dd}, J=7.9,1.2$ $\mathrm{Hz}, 1 \mathrm{H}), 7.57(\mathrm{dd}, J=1.8,0.8 \mathrm{~Hz}, 1 \mathrm{H}), 7.30-7.18(\mathrm{~m}, 2 \mathrm{H}), 7.17(\mathrm{dd}, J=3.5,0.8 \mathrm{~Hz}, 1 \mathrm{H}), 6.93-6.83(\mathrm{~m}, 1 \mathrm{H}), 6.50(\mathrm{dd}, J=$ $3.5,1.7 \mathrm{~Hz}, 1 \mathrm{H}), 4.98-4.87(\mathrm{~m}, 2 \mathrm{H}), 4.46(\mathrm{t}, J=7.0 \mathrm{~Hz}, 2 \mathrm{H}), 2.91-2.81(\mathrm{~m}, 2 \mathrm{H}), 2.55(\mathrm{t}, J=6.9 \mathrm{~Hz}, 2 \mathrm{H}), 2.39-2.29(\mathrm{~m}, 2 \mathrm{H})$ ppm; ${ }^{13} \mathrm{C}$ NMR $\left(101 \mathrm{MHz}, \mathrm{CDCl}_{3}\right) \delta=158.7,146.3,144.8,144.4,144.3,139.5,129.4,128.4,127.8,117.9,112.3,111.8,100.4$, $63.4,39.5,36.7,35.2 \mathrm{ppm}$.

\section{Procedure for Synthesis of tert-Butyl((5-(2-iodophenyl)-3-methylenepentyl)oxy)diphenylsilane (1h)}<smiles>C=C(CCO)CCc1ccccc1I</smiles>

S-4

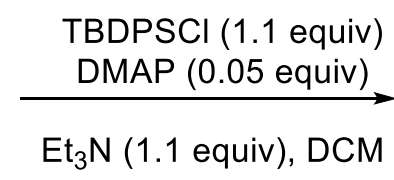

$\mathrm{Et}_{3} \mathrm{~N}$ (1.1 equiv), DCM

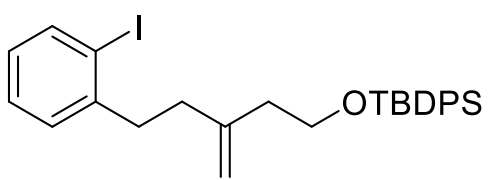

$1 \mathrm{~h}$

To a stirred solution of the homoallylic alcohol S-4 ( $8.0 \mathrm{mmol}, 1$ equiv), $\mathrm{Et}_{3} \mathrm{~N}(1.3 \mathrm{~mL}, 8.8 \mathrm{mmol}, 1.1$ equiv) and DMAP (50 $\mathrm{mg}, 0.4 \mathrm{mmol}, 5 \mathrm{~mol} \%)$ in $\mathrm{CH}_{2} \mathrm{Cl}_{2}(25 \mathrm{~mL})$, TBDPSCl $(1.32 \mathrm{~g}, 8.8 \mathrm{mmol}, 1.1$ equiv) was added at room temperature. After stirring for $12 \mathrm{~h}$, water was added $(30 \mathrm{~mL})$, and the aqueous layer was extracted twice with $\mathrm{CH}_{2} \mathrm{Cl}_{2}$. The combine organic 
layers were washed with brine, dried over anhydrous $\mathrm{Na}_{2} \mathrm{SO}_{4}$, filtered and evaporated to dryness. The crude residue was purified by column chromatography (petroleum ether/ethyl acetate $=20: 1$ ) on silica gel to give the product $\mathbf{1 h}$ as a colorless oil (2.16 g, $4.0 \mathrm{mmol}, 50 \%$ yield). ${ }^{1} \mathrm{H}$ NMR $\left(400 \mathrm{MHz}, \mathrm{CDCl}_{3}\right) \delta=7.79(\mathrm{dd}, J=7.9,1.3 \mathrm{~Hz}, 1 \mathrm{H}), 7.73-7.64(\mathrm{~m}, 4 \mathrm{H}), 7.46-$ $7.32(\mathrm{~m}, 6 \mathrm{H}), 7.28-7.19(\mathrm{~m}, 1 \mathrm{H}), 7.13(\mathrm{dd}, J=7.6,1.8 \mathrm{~Hz}, 1 \mathrm{H}), 6.86(\mathrm{td}, J=7.6,1.8 \mathrm{~Hz}, 1 \mathrm{H}), 4.88-4.73(\mathrm{~m}, 2 \mathrm{H}), 3.80(\mathrm{t}, J=$ $7.0 \mathrm{~Hz}, 2 \mathrm{H}), 2.83-2.71(\mathrm{~m}, 2 \mathrm{H}), 2.36(\mathrm{t}, J=6.9 \mathrm{~Hz}, 2 \mathrm{H}), 2.26-2.17(\mathrm{~m}, 2 \mathrm{H}), 1.05(\mathrm{~s}, 9 \mathrm{H}) \mathrm{ppm} ;{ }^{13} \mathrm{C} \mathrm{NMR}\left(101 \mathrm{MHz}, \mathrm{CDCl}_{3}\right)$ $\delta=145.8,144.6,139.4$ (2C), 135.6 (4C), 134.0, 129.6 (2C), 129.4, 128.3, 127.71, 127.66 (4C), 111.5, 100.5, 63.0, 39.6, 39.3, $36.8,26.9(3 \mathrm{C}), 19.3 \mathrm{ppm}$.

(3) Procedure for Synthesis of $N$-Benzyl-N-(2-iodobenzyl)-2-methylprop-2-en-1-amine (10)<smiles>N#Cc1ccc(I)c(O)c1</smiles>

S-5

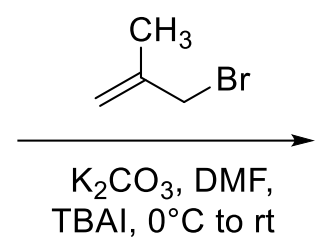

$\mathrm{K}_{2} \mathrm{CO}_{3}, \mathrm{DMF}$
TBAI, $0^{\circ} \mathrm{C}$ to rt

To a solution of 1-(3-hydroxy-4-iodophenyl)ethan-1-one $\mathbf{S - 5}{ }^{11}$ (2.36 g, 9 mmol, 1.0 equiv) and potassium carbonate (2.48 g, $18.0 \mathrm{mmol}, 2.0$ equiv) in $\mathrm{DMF}(30 \mathrm{~mL})$ at $0{ }^{\circ} \mathrm{C}$ was added dropwise 3-bromo-2-methylprop-1-ene $(1.34 \mathrm{~g}, 1.0 \mathrm{~mL}, 9.9 \mathrm{mmol}$, 1.1 equiv). After stirring at room temperature overnight, the mixture was quenched with water and extracted with ethyl acetate. The extract was washed with brine, dried over anhydrous $\mathrm{Na}_{2} \mathrm{SO}_{4}$, and concentrated. Purification by column chromatography (petroleum ether/ethyl acetate $=20: 1$ to $10: 1)$ on silica gel gave pure product 10 as a yellow oil (2.73 g, $8.64 \mathrm{mmol}, 96 \%$ yield) . ${ }^{1} \mathrm{H}$ NMR $\left(400 \mathrm{MHz} \mathrm{CDCl}_{3}\right) \delta=8.40(\mathrm{~d}, \mathrm{~J}=2.1 \mathrm{~Hz}, 1 \mathrm{H}), 7.92(\mathrm{dd}, \mathrm{J}=8.6,2.2 \mathrm{~Hz}, 1 \mathrm{H}), 6.82(\mathrm{~d}, \mathrm{~J}=8.6 \mathrm{~Hz}, 1 \mathrm{H}), 5.22-5.18$ $(\mathrm{m}, 1 \mathrm{H}), 5.07-5.04(\mathrm{~m}, 1 \mathrm{H}), 4.56(\mathrm{~s}, 2 \mathrm{H}), 2.55(\mathrm{~s}, 3 \mathrm{H}), 1.88(\mathrm{~s}, 3 \mathrm{H}) \mathrm{ppm} ;{ }^{13} \mathrm{C} \mathrm{NMR}\left(101 \mathrm{MHz}, \mathrm{CDCl}_{3}\right) \delta=195.5,160.8$, $140.2,139.5,131.9,130.4,113.5,111.2,86.4,72.8,26.3,19.4$ ppm.

(4) Procedure for Synthesis of $N$-Benzyl-N-(2-iodobenzyl)-2-methylprop-2-en-1-amine (1s)

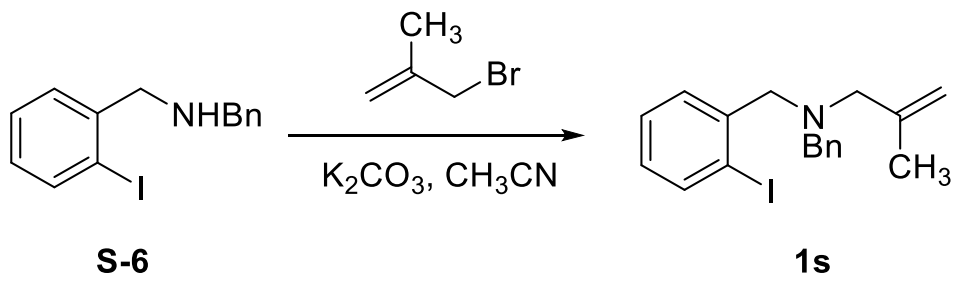

To a solution of $\mathrm{N}$-benzyl-1-(2-iodophenyl)methanamine $\mathbf{S - 5} \mathbf{5}^{12}$ (2.15 g, $6.7 \mathrm{mmol}, 1.0$ equiv) and potassium carbonate (1.38 $\mathrm{g}, 10.0 \mathrm{mmol}, 1.5$ equiv) in acetonitrile $(30 \mathrm{~mL})$ was added dropwise 3-bromo-2-methylprop-1-ene $(0.9 \mathrm{~g}, 0.7 \mathrm{~mL}, 6.7 \mathrm{mmol}$, 1.0 equiv). After stirring at room temperature overnight, the mixture was quenched with water and extracted with ethyl acetate. The extract was washed with brine, dried over anhydrous $\mathrm{Na}_{2} \mathrm{SO}_{4}$, and concentrated. Purification by column chromatography (petroleum ether/ethyl acetate $=10: 1$ to $2: 1)$ on silica gel gave pure product $1 \mathrm{~s}$ as a colorless oil $(1.26 \mathrm{~g}, 3.35 \mathrm{mmol}, 50 \%$ yield). ${ }^{1} \mathrm{H} \mathrm{NMR}\left(400 \mathrm{MHz}, \mathrm{CDCl}_{3}\right) \delta=7.78(\mathrm{dd}, J=7.9,1.2 \mathrm{~Hz}, 1 \mathrm{H}), 7.65(\mathrm{dd}, J=7.7,1.7 \mathrm{~Hz}, 1 \mathrm{H}), 7.42-7.35(\mathrm{~m}, 2 \mathrm{H}), 7.35-$ $7.26(\mathrm{~m}, 3 \mathrm{H}), 7.27-7.18(\mathrm{~m}, 1 \mathrm{H}), 6.91(\mathrm{td}, J=7.6,1.7 \mathrm{~Hz}, 1 \mathrm{H}), 5.04-4.80(\mathrm{~m}, 2 \mathrm{H}), 3.58(\mathrm{~s}, 2 \mathrm{H}), 3.56(\mathrm{~s}, 2 \mathrm{H}), 2.97(\mathrm{~s}, 2 \mathrm{H})$, 1.77 (s, 3H) ppm; ${ }^{13} \mathrm{C}$ NMR (101 $\left.\mathrm{MHz}, \mathrm{CDCl}_{3}\right) \delta=143.5,141.8,139.4,139.2,129.8,128.7$ (2C), 128.5, 128.3 (2C), 128.2, $126.9,113.1,100.1,62.4,60.8,58.2,21.0 \mathrm{ppm}$. 


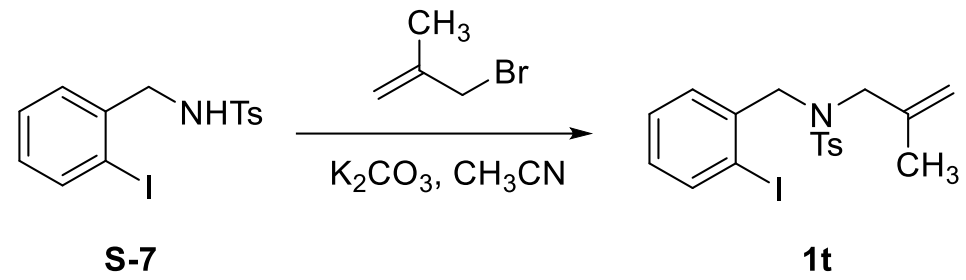

To a solution of $\mathbf{S - 7}^{13}$ (1.26 g, $3.25 \mathrm{mmol}, 1.0$ equiv) and potassium carbonate ( $0.69 \mathrm{~g}, 5.0 \mathrm{mmol}, 1.54$ equiv) in acetonitrile $(30 \mathrm{~mL})$ was added dropwise 3-bromo-2-methylprop-1-ene $(0.54 \mathrm{~g}, 0.4 \mathrm{~mL}, 4.0 \mathrm{mmol}, 1.23$ equiv). After stirring at room temperature overnight, the mixture was quenched with water and extracted with ethyl acetate. The extract was washed with brine, dried over anhydrous $\mathrm{Na}_{2} \mathrm{SO}_{4}$, and concentrated. Purification by column chromatography (petroleum ether/ethyl acetate $=10: 1$ to 2:1) on silica gel gave pure product $1 \mathrm{t}$ as a colorless oil $(0.85 \mathrm{~g}, 1.92 \mathrm{mmol}, 59 \%$ yield $) .{ }^{1} \mathrm{H} \mathrm{NMR}(400 \mathrm{MHz}$, $\left.\mathrm{CDCl}_{3}\right) \delta=7.79-7.70(\mathrm{~m}, 3 \mathrm{H}), 7.46(\mathrm{dd}, J=7.8,1.6 \mathrm{~Hz}, 1 \mathrm{H}), 7.36-7.25(\mathrm{~m}, 3 \mathrm{H}), 6.93(\mathrm{td}, J=7.6,1.7 \mathrm{~Hz}, 1 \mathrm{H}), 4.77-4.61(\mathrm{~m}$, 2H), 4.36 (s, 2H), 3.74 (s, 2H), 2.44 (s, 3H), 1.53 (s, 3H) ppm; $\left.{ }^{13} \mathrm{C} \mathrm{NMR} \mathrm{(101} \mathrm{MHz,} \mathrm{CDCl}_{3}\right) \delta=143.5,139.5,139.3,138.3$, 136.8, 129.7 (2C), 129.2, 129.1, 128.4, 127.3 (2C), 115.2, 98.4, 56.2, 54.9, 21.6, $20.0 \mathrm{ppm}$.

(6) Procedure for Synthesis 1-iodo-2-((5-methylhex-5-en-1-yl)oxy)benzene (1v)

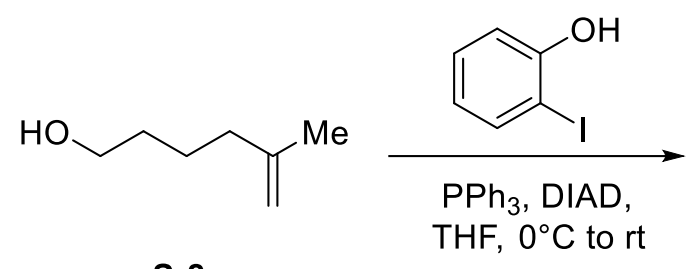

S-8

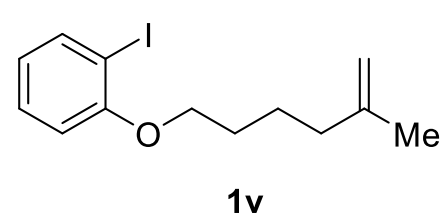

To a stirred solution of alcohol S-8 ${ }^{14}$ (1.92 g, $16.8 \mathrm{mmol}, 1.0$ equiv), 2-iodophenol (3.69 g, $16.8 \mathrm{mmol}, 1.0$ equiv), and $\mathrm{PPh}_{3}$ (4.40 g, $16.8 \mathrm{mmol}, 1.0$ equiv) in THF $(50 \mathrm{~mL})$ was added DIAD $\left(3.3 \mathrm{ml}, 16.8 \mathrm{mmol}, 1.0\right.$ equiv) dropwise at $0{ }^{\circ} \mathrm{C}$ under $\mathrm{N}_{2}$. After stirring at room temperature overnight, the mixture was quenched with water and extracted with ethyl acetate. The extract was washed with brine, dried over anhydrous $\mathrm{Na}_{2} \mathrm{SO}_{4}$, and concentrated. Purification through column chromatography (petroleum ether/ethyl acetate $=20: 1)$ on silica gel gave the pure product $1 \mathbf{v}$ as a colorless oil $(3.72 \mathrm{~g}, 11.76 \mathrm{mmol}, 70 \%$ yield). ${ }^{1} \mathrm{H}$ NMR $\left(400 \mathrm{MHz}, \mathrm{CDCl}_{3}\right) \delta=7.76(\mathrm{dd}, \mathrm{J}=7.8,1.7 \mathrm{~Hz}, 1 \mathrm{H}), 7.34-7.20(\mathrm{~m}, 1 \mathrm{H}), 6.79(\mathrm{dd}, \mathrm{J}=8.2,1.3 \mathrm{~Hz}, 1 \mathrm{H}), 6.68(\mathrm{td}, \mathrm{J}$ $=7.6,1.4 \mathrm{~Hz}, 1 \mathrm{H}), 4.81-4.65(\mathrm{~m}, 2 \mathrm{H}), 4.01(\mathrm{t}, \mathrm{J}=6.1 \mathrm{~Hz}, 2 \mathrm{H}), 2.11(\mathrm{t}, \mathrm{J}=7.4 \mathrm{~Hz}, 2 \mathrm{H}), 1.90-1.61(\mathrm{~m}, 7 \mathrm{H}) \mathrm{ppm} ;{ }^{13} \mathrm{C} \mathrm{NMR}$ $\left(101 \mathrm{MHz}, \mathrm{CDCl}_{3}\right) \delta=157.6,145.6,139.4,129.4,122.3,112.0,110.2,86.7,69.0,37.4,28.7,24.0,22.4 \mathrm{ppm}$.

\section{General Procedure for the Ni-Catalyzed Reductive Arylalkylation of Unactivated Olefins}<smiles>[R]C=C([R])C[Y]=[V]c1cccc([R3])c1I</smiles>

1<smiles>[R]C([R])C(=O)ON1C(=O)c2ccccc2C1=O</smiles>

2
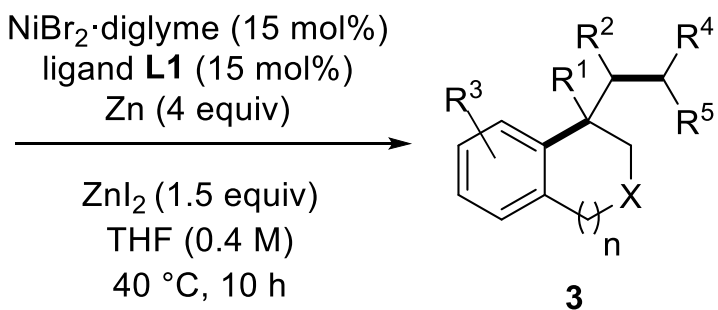

3<smiles>N#CN=C(N)c1ccccn1</smiles>

L1

A sealed test tube charged with $\mathrm{NiBr}_{2} \bullet$ diglyme (10.6 mg, $\left.0.03 \mathrm{mmol}, 15 \mathrm{~mol} \%\right)$, ligand $\mathbf{L 1}$ (4.5 mg, $\left.0.03 \mathrm{mmol}, 15 \mathrm{~mol} \%\right)$, $\mathrm{ZnI}_{2}$ (96 mg, $0.3 \mathrm{mmol}, 1.5$ equiv), Zn-powder (52 mg, $0.8 \mathrm{mmol}, 4.0$ equiv) and a stir bar was evacuated and filled with nitrogen (three cycles). Next, alkenes 1 ( $0.2 \mathrm{mmol}, 1.0$ equiv), NHP Esters 2 ( $0.4 \mathrm{mmol}, 2.0$ equiv) and THF $(0.5 \mathrm{~mL})$ were added and the resulting mixture was stirred at $40{ }^{\circ} \mathrm{C}$ for 10 hours. The mixture was then filtered through a pad of Celite and concentrated under reduced pressure. The residue was purified by column chromatography (petroleum ether/ethyl acetate) on 
silica gel to afford the corresponding products.

\section{Analytical Data for the Products}

Compounds 3a, 3d and $\mathbf{3 r}$ are known compounds and their NMR-data are consistent with these reported in the literature. ${ }^{15}$

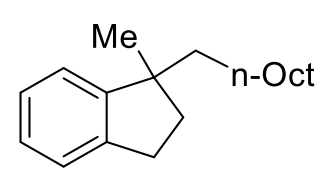

$3 a$

1-(1-Methyl-2,3-dihydro-1H-inden-1-yl)nonan-2-one (3a) was isolated through column chromatography on silica gel with petroleum ether as a colorless oil (37 mg, $68 \%)$. ${ }^{1} \mathrm{H}$ NMR: (400 MHz, Chloroform- $d$ ) $\delta=7.22-7.06(\mathrm{~m}, 4 \mathrm{H}), 2.91-2.77(\mathrm{~m}, 2 \mathrm{H}), 2.10-1.94(\mathrm{~m}, 1 \mathrm{H}), 1.87-1.75(\mathrm{~m}, 1 \mathrm{H})$, 1.62-1.42 (m, 2H), 1.38-1.12 (m, 13H), 1.22 (s, 3H), 0.93-0.77 (m, 4H) ppm. ${ }^{13} \mathrm{C}$ NMR $(101 \mathrm{MHz}$, Chloroform- $d$ ): $\delta=151.8,143.2,126.13,126.10,124.5,122.7,47.4,41.5,38.6,31.9,30.5,30.3,29.7$

(2C), 29.4, 26.8, 24.9, 22.7, $14.2 \mathrm{ppm}$.

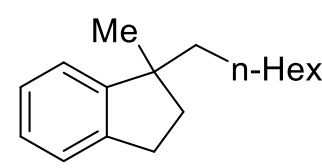

$3 b$

1-(1-Methyl-2,3-dihydro-1H-inden-1-yl)octan-3-one (3b) was isolated through column chromatography on silica gel with petroleum ether as a colorless oil (29 mg, $62 \%)$. ${ }^{1} \mathrm{H}$ NMR: (400 MHz, Chloroform- $d$ ) $\delta=7.22-7.07(\mathrm{~m}, 4 \mathrm{H}), 2.92-2.82(\mathrm{~m}, 2 \mathrm{H}), 2.07-1.96(\mathrm{~m}, 1 \mathrm{H}), 1.87-1.75(\mathrm{~m}, 1 \mathrm{H})$, 1.63-1.44 (m, 2H), 1.35-1.12 (m, 10H), $1.22(\mathrm{~s}, 3 \mathrm{H}), 0.86(\mathrm{t}, J=6.8 \mathrm{~Hz}, 3 \mathrm{H}) \mathrm{ppm} .{ }^{13} \mathrm{C} \mathrm{NMR}(101$

MHz, Chloroform- $d$ ): $\delta=151.8,143.2,126.11,126.08,124.5,122.7,47.3,41.5,38.6,34.0,30.4,30.3$, 29.4, 26.8, 25.0, 22.7, 14.2 ppm. HRMS (EI): calcd. for $\mathrm{C}_{17} \mathrm{H}_{26}{ }^{+}\left[\mathrm{M}^{+}\right]$: 230.2029, found: 230.2027.

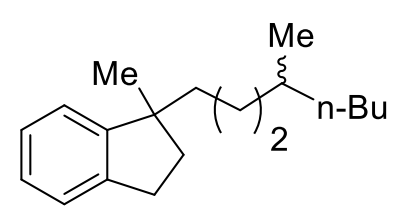

$3 c$

1-Methyl-1-(4-methyloctyl)-2,3-dihydro-1H-indene (3c) was isolated through column chromatography on silica gel with petroleum ether as a colorless oil $(34 \mathrm{mg}, 65 \%, d r=1: 1) .{ }^{1} \mathrm{H}$ NMR: (400 MHz, Chloroform- $d$ ) $\delta=7.23-7.05$ (m, 4H), 2.92-2.83 (m, 2H), 2.07-1.95 (m, 1H), 1.87-1.76 (m, 1H), 1.63-1.41 (m, 2H), 1.38-1.00 (m, 11H), $0.87(\mathrm{t}, J=6.8 \mathrm{~Hz}, 3 \mathrm{H}), 0.82(\mathrm{~d}, J=$ $2.1 \mathrm{~Hz}, 1.5 \mathrm{H})[0.80(\mathrm{~d}, J=2.0 \mathrm{~Hz}, 1.5 \mathrm{H})] \mathrm{ppm}$. Inside the brackets is the chemical shift of another diastereomer. ${ }^{13} \mathrm{C}$ NMR (101 MHz, Chloroform-d): $\delta$ (mixture of two diastereomers) $=151.9$, 143.2 , 126.12, 126.10, 124.5, 122.6, 47.4, 41.7, 38.6, 37.9, 36.9, 36.8, 32.64, 32.62, 30.3, 29.4, 26.78, 26.75, 23.1, 22.4, 19.8, 19.7, 14.2 ppm. HRMS (EI): calcd. for $\mathrm{C}_{19} \mathrm{H}_{30}{ }^{+}\left[\mathrm{M}^{+}\right]$: 258.2342, found: 258.2342 .

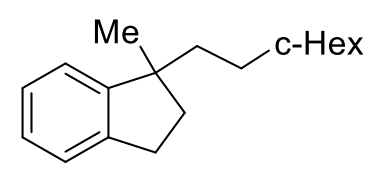

$3 d$

1-(2-Cyclohexylethyl)-1-methyl-2,3-dihydro-1H-indene (3d) was isolated through column chromatography on silica gel with petroleum ether as a colorless oil (31 mg, $63 \%) .{ }^{1} \mathrm{H}$ NMR: (400 $\mathrm{MHz}$, Chloroform- $d$ ) $\delta=7.22-7.03(\mathrm{~m}, 4 \mathrm{H}), 2.93-2.79(\mathrm{~m}, 2 \mathrm{H}), 2.05-1.93(\mathrm{~m}, 1 \mathrm{H}), 1.85-1.74(\mathrm{~m}$, $1 \mathrm{H}), 1.72-1.47(\mathrm{~m}, 7 \mathrm{H}), 1.28-1.02(\mathrm{~m}, 6 \mathrm{H}), 1.22(\mathrm{~s}, 3 \mathrm{H}), 0.93-0.76(\mathrm{~m}, 2 \mathrm{H}) \mathrm{ppm} .{ }^{13} \mathrm{C}$ NMR $(101$ MHz, Chloroform- $d): \delta=151.9,143.2,126.1$ (2C), 124.5, 122.6, 47.3, 38.6, 38.5, 38.4, 33.53, 33.47, 32.5, 30.3, 26.82, 26.77, 26.48, 26.47 ppm. HRMS (EI): calcd. for $\mathrm{C}_{18} \mathrm{H}_{26}{ }^{+}\left[\mathrm{M}^{+}\right]$: 242.2035, found: 242.2036 .

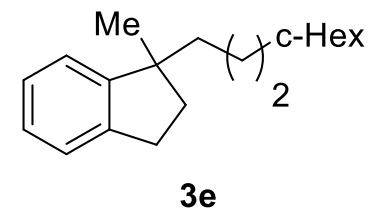

38.6, 38.3, 37.6, 33.6, 33.4, 30.3, 26.80, 26.79, 26.5 (2C), 22.1 ppm. HRMS (EI): calcd. for $\mathrm{C}_{19} \mathrm{H}_{28}{ }^{+}\left[\mathrm{M}^{+}\right]: 256.2186$, found: 256.2187.
1-(3-Cyclohexylpropyl)-1-methyl-2,3-dihydro-1H-indene (3e) was isolated through column chromatography on silica gel with petroleum ether as a colorless oil (38 mg, $74 \%)$. ${ }^{1} \mathrm{H}$ NMR: (400 $1 \mathrm{H}), 1.70-1.57(\mathrm{~m}, 5 \mathrm{H}), 1.57-1.39(\mathrm{~m}, 2 \mathrm{H}), 1.36-1.05(\mathrm{~m}, 8 \mathrm{H}), 1.22(\mathrm{~s}, 3 \mathrm{H}), 0.91-0.74(\mathrm{~m}, 2 \mathrm{H}) \mathrm{ppm}$.

${ }^{13} \mathrm{C}$ NMR (101 MHz, Chloroform- $\left.d\right): \delta=151.9,143.2,126.12,126.10,124.5,122.6,47.4,41.7$, $\mathrm{MHz}$, Chloroform- $d$ ) $\delta=7.21-7.06(\mathrm{~m}, 4 \mathrm{H}), 2.93-2.80(\mathrm{~m}, 2 \mathrm{H}), 2.05-1.95(\mathrm{~m}, 1 \mathrm{H}), 1.86-1.75(\mathrm{~m}$, 


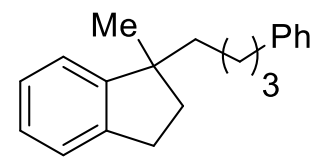

$3 f$

1-Methyl-1-(4-phenylbutyl)-2,3-dihydro-1H-indene (3f) was isolated through column chromatography on silica gel with petroleum ether as a colorless oil (39 mg, $73 \%$ ). ${ }^{1} \mathrm{H}$ NMR: (400 MHz, Chloroformd) $\delta=7.29-7.22(\mathrm{~m}, 2 \mathrm{H}), 7.20-7.03(\mathrm{~m}, 7 \mathrm{H}), 2.91-2.83(\mathrm{~m}, 2 \mathrm{H}), 2.62-2.52(\mathrm{~m}, 2 \mathrm{H}), 2.04-1.95(\mathrm{~m}, 1 \mathrm{H})$, 1.86-1.77 (m, 1H), 1.66-1.49 (m, 4H), 1.42-1.20 (m, 2H), $1.22(\mathrm{~s}, 3 \mathrm{H}) \mathrm{ppm} .{ }^{13} \mathrm{C}$ NMR $(101 \mathrm{MHz}$, Chloroform- $d$ ): $\delta=151.7,143.2,142.8,128.4(2 \mathrm{C}), 128.3(2 \mathrm{C}), 126.2,126.1,125.6,124.5,122.7,47.3$, 41.2, 38.6, 36.0, 32.3, 30.3, 26.8, 24.7 ppm. HRMS (EI): calcd. for $\mathrm{C}_{20} \mathrm{H}_{24}{ }^{+}\left[\mathrm{M}^{+}\right]$: 264.1873, found: 264.1875 .

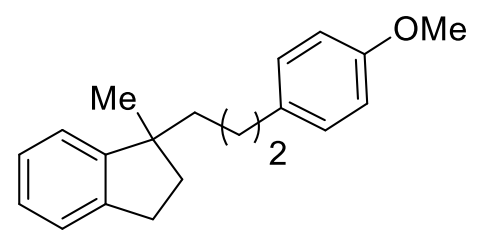

$3 g$

1-(3-(4-Methoxyphenyl)propyl)-1-methyl-2,3-dihydro-1H-indene (3g) was isolated through column chromatography on with petroleum ether to the mixture eluent of petroleum ether and ethyl acetate (20:1) as a colorless oil (32 mg, $58 \%) .{ }^{1} \mathrm{H}$ NMR: (400 MHz, Chloroform-d) $\delta=7.26-7.07$ (m, 6H), 6.90-6.81 (m, 2H), $3.83(\mathrm{~s}, 3 \mathrm{H}), 2.98-2.85(\mathrm{~m}, 2 \mathrm{H})$, 2.61-2.47 (m, 2H), 2.13-1.99 (m, 1H), 1.93-1.79 (m, 1H), 1.74-1.49 (m, 4H), $1.27(\mathrm{~s}, 3 \mathrm{H})$ ppm. ${ }^{13} \mathrm{C}$ NMR (101 MHz, Chloroform- $d$ ): $\delta=157.7,151.5,143.1,134.7,129.2(2 \mathrm{C}), 126.2$, 126.1, 124.5, 122.6, 113.7 (2C), 55.3, 47.3, 41.0, 38.6, 35.7, 30.3, 27.1, 26.8 ppm. HRMS (EI): calcd. for $\mathrm{C}_{20} \mathrm{H}_{24} \mathrm{O}^{+}\left[\mathrm{M}^{+}\right]$: 280.1822 , found: 280.1823 .

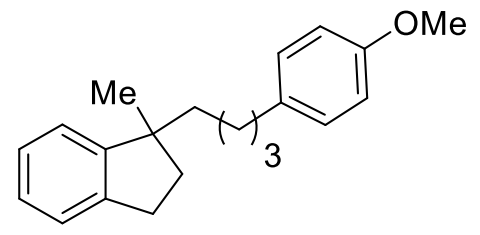

$3 h$

1-(4-(4-Methoxyphenyl)butyl)-1-methyl-2,3-dihydro-1H-indene (3h) was isolated through column chromatography on with petroleum ether to the mixture eluent of petroleum ether and ethyl acetate (20:1) as a colorless oil $(41 \mathrm{mg}, 70 \%) .{ }^{1} \mathrm{H}$ NMR: $(400 \mathrm{MHz}$, Chloroform-d) $\delta=7.21-7.06(\mathrm{~m}, 4 \mathrm{H}), 7.05(\mathrm{~d}, J=8.3 \mathrm{~Hz}, 2 \mathrm{H}), 6.80(\mathrm{~d}, J=8.3 \mathrm{~Hz}, 2 \mathrm{H})$, $3.77(\mathrm{~s}, 3 \mathrm{H}), 2.93-2.81(\mathrm{~m}, 2 \mathrm{H}), 2.58-2.44(\mathrm{~m}, 2 \mathrm{H}), 2.07-1.94(\mathrm{~m}, 1 \mathrm{H}), 1.87-1.75(\mathrm{~m}, 1 \mathrm{H})$, 1.67-1.45 (m, 4H), 1.42-1.27 (m, 2H), $1.22(\mathrm{~s}, 3 \mathrm{H})$ ppm. ${ }^{13} \mathrm{C}$ NMR (101 MHz, Chloroformd): $\delta=157.6,151.7,143.2,135.0,129.2$ (2C), 126.2, 126.1, 124.5, 122.7, 113.7 (2C), 55.3, 47.3, 41.2, 38.6, 35.0, 32.6, 30.3, 26.8, 24.7 ppm. HRMS (EI): calcd. for $\mathrm{C}_{21} \mathrm{H}_{26} \mathrm{O}$ [M]: 294.1978, found: 294.1975 .

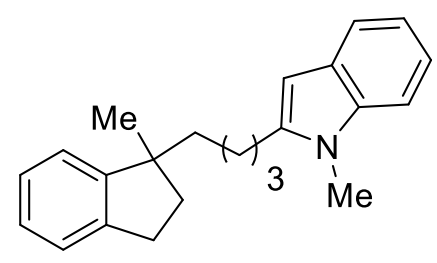

$3 \mathbf{i}$

1-Methyl-2-(4-(1-methyl-2,3-dihydro-1H-inden-1-yl)butyl)-1H-indole (3i) was isolated through column chromatography on silica gel with petroleum ether to the mixture eluent of petroleum ether and ethyl acetate (20:1) as a colorless oil (37 mg, $58 \%) .{ }^{1} \mathrm{H}$ NMR: (400 MHz, Chloroform- $d$ ) $\delta=7.60-7.52(\mathrm{~m}, 1 \mathrm{H}), 7.30-7.03(\mathrm{~m}, 7 \mathrm{H}), 6.74(\mathrm{~s}, 1 \mathrm{H}), 3.71(\mathrm{~s}, 3 \mathrm{H})$, 2.92-2.83 (m, 2H), 2.75-2.65 (m, 2H), 2.08-1.96 (m, 1H), 1.88-1.76 (m, 1H), 1.72-1.58 (m, $3 \mathrm{H}), 1.59-1.49(\mathrm{~m}, 1 \mathrm{H}), 1.48-1.37(\mathrm{~m}, 1 \mathrm{H}), 1.36-1.18(\mathrm{~m}, 1 \mathrm{H}), 1.23(\mathrm{~s}, 3 \mathrm{H}) \mathrm{ppm} .{ }^{13} \mathrm{C}$ NMR (101 MHz, Chloroform- $d$ ): $\delta=151.8,143.3,137.0,127.9,126.2,126.1,125.9,124.5,122.7$, 121.4, 119.1, 118.4, 115.5, 109.1, 47.4, 41.3, 38.7, 32.6, 31.2, 30.3, 26.8, 25.04, 25.00 ppm. HRMS (ESI): calcd. for $\mathrm{C}_{23} \mathrm{H}_{28} \mathrm{~N}^{+}$ $[\mathrm{M}+\mathrm{H}]^{+}:$318.2216, found: 318.2217 .

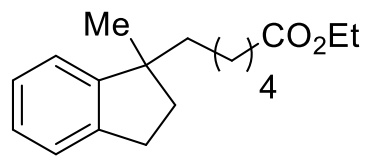

3j

Ethyl 6-(1-methyl-2,3-dihydro-1H-inden-1-yl)hexanoate (3j) was isolated through column chromatography on silica gel with petroleum ether to the mixture eluent of petroleum ether and ethyl acetate (20:1) as a colorless oil (36 mg, $66 \%)$. ${ }^{1} \mathrm{H}$ NMR: (400 MHz, Chloroform-d) $\delta=$ 7.23-7.05 (m, 4H), $4.11(\mathrm{q}, J=7.1 \mathrm{~Hz}, 2 \mathrm{H}), 2.93-2.81(\mathrm{~m}, 2 \mathrm{H}), 2.25(\mathrm{t}, J=7.5 \mathrm{~Hz}, 2 \mathrm{H}), 2.06-$ $1.94(\mathrm{~m}, 1 \mathrm{H}), 1.87-1.75(\mathrm{~m}, 1 \mathrm{H}), 1.66-1.54(\mathrm{~m}, 3 \mathrm{H}), 1.52-1.43(\mathrm{~m}, 1 \mathrm{H}), 1.35-1.14(\mathrm{~m}, 4 \mathrm{H}), 1.24$ (t, $J=7.2 \mathrm{~Hz}, 3 \mathrm{H}), 1.22$ (s, 3H) ppm. ${ }^{13} \mathrm{C}$ NMR (101 MHz, Chloroform-d): $\delta=173.9,151.6,143.2,126.2,126.1,124.5,122.6$, 60.2, 47.3, 41.2, 38.6, 34.4, 30.3, 29.9, 26.8, 25.0, 24.6, 14.3 ppm. HRMS (ESI): calcd. for $\mathrm{C}_{18} \mathrm{H}_{27} \mathrm{O}_{2}{ }^{+}[\mathrm{M}+\mathrm{H}]^{+}: 275.2006$, found: 275.2003 . 


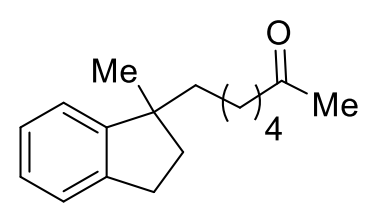

3k

7-(1-Methyl-2,3-dihydro-1H-inden-1-yl)heptan-2-one (3k) was isolated through column chromatography on silica gel with petroleum ether to the mixture eluent of petroleum ether and ethyl acetate (20:1) as a colorless oil (32 mg, $65 \%)$. ${ }^{1} \mathrm{H}$ NMR: (400 MHz, Chloroform- $d$ ) $\delta=$ 7.21-7.05 (m, 4H), 2.90-2.83 (m, 2H), 2.42-2.33 (m, 2H), $2.11(\mathrm{~s}, 3 \mathrm{H}), 2.04-1.95(\mathrm{~m}, 1 \mathrm{H}), 1.86-$ $1.76(\mathrm{~m}, 1 \mathrm{H}), 1.63-1.43(\mathrm{~m}, 4 \mathrm{H}), 1.32-1.14(\mathrm{~m}, 4 \mathrm{H}), 1.22(\mathrm{~s}, 3 \mathrm{H}) \mathrm{ppm} .{ }^{13} \mathrm{C}$ NMR $(101 \mathrm{MHz}$, Chloroform- $d$ ): $\delta=209.3,151.5,143.2,126.2,126.1,124.5,122.6,47.3,43.8,41.2,38.6,30.3$, 29.92, 29.88, 26.8, 24.7, 23.8 ppm. HRMS (ESI): calcd. for $\mathrm{C}_{17} \mathrm{H}_{25} \mathrm{O}^{+}[\mathrm{M}+\mathrm{H}]^{+}: 245.1900$, found: 245.1901 .

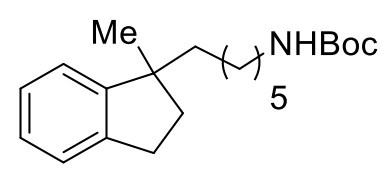

31

tert-Butyl (5-(1-methyl-2,3-dihydro-1H-inden-1-yl)pentyl)carbamate (3I) was isolated through column chromatography on silica gel with petroleum ether to the mixture eluent of petroleum ether and ethyl acetate $\quad(20: 1)$ as a colorless oil (44 mg, 69\%). ${ }^{1} \mathrm{H}$ NMR: (400 MHz, Chloroformd) $\delta=7.22-7.05(\mathrm{~m}, 4 \mathrm{H}), 4.49(\mathrm{~s}, 1 \mathrm{H}), 3.13-2.99(\mathrm{~m}, 2 \mathrm{H}), 2.91-2.80(\mathrm{~m}, 2 \mathrm{H}), 2.07-1.93(\mathrm{~m}, 1 \mathrm{H})$, $1.88-1.74(\mathrm{~m}, 1 \mathrm{H}), 1.63-1.53(\mathrm{~m}, 1 \mathrm{H}), 1.51-1.38(\mathrm{~m}, 2 \mathrm{H}), 1.43(\mathrm{~s}, 9 \mathrm{H}), 1.36-1.13(\mathrm{~m}, 5 \mathrm{H}), 1.22(\mathrm{~s}$, $3 \mathrm{H})$ ppm. ${ }^{13} \mathrm{C}$ NMR (101 MHz, Chloroform- $d$ ): $\delta=156.0,151.6,143.2,126.2,126.1,124.5,122.6,79.0,47.3,41.4,40.6,38.6$, 30.3, 30.1, 28.5 (3C), 27.6, 26.8, 24.7 ppm. HRMS (ESI): calcd. for $\mathrm{C}_{20} \mathrm{H}_{32} \mathrm{NO}_{2}{ }^{+}[\mathrm{M}+\mathrm{H}]^{+}:$318.2428, found: 318.2426 .

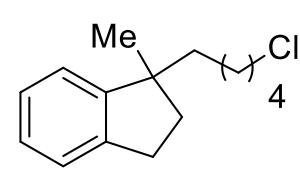

$3 m$

1-(5-Chloropentyl)-1-methyl-2,3-dihydro-1H-indene (3m) was isolated through column chromatography on silica gel with petroleum ether as a colorless oil (34 mg, $71 \%) .{ }^{1} \mathrm{H}$ NMR: (400 MHz, Chloroform- $d$ ) $\delta=7.21-7.07(\mathrm{~m}, 4 \mathrm{H}), 3.52-3.45(\mathrm{~m}, 2 \mathrm{H}), 2.91-2.84(\mathrm{~m}, 2 \mathrm{H}), 2.05-1.96(\mathrm{~m}, 1 \mathrm{H})$, $1.87-1.70(\mathrm{~m}, 3 \mathrm{H}), 1.64-1.46(\mathrm{~m}, 2 \mathrm{H}), 1.44-1.29(\mathrm{~m}, 3 \mathrm{H}), 1.28-1.18(\mathrm{~m}, 1 \mathrm{H}), 1.23(\mathrm{~s}, 3 \mathrm{H}) \mathrm{ppm} .{ }^{13} \mathrm{C}$ NMR (101 MHz, Chloroform- $d$ ): $\delta=151.5,143.2,126.24,126.15,124.5,122.6,47.3,45.2,41.3,38.6$, 32.6, 30.3, 27.7, 26.9, 24.3 ppm. HRMS (EI): calcd. for $\mathrm{C}_{15} \mathrm{H}_{21} \mathrm{Cl}^{+}\left[\mathrm{M}^{+}\right]$: 236.1326, found: 236.1327.

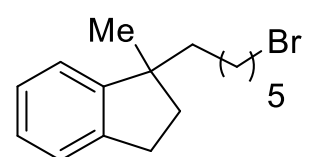

$3 n$

1-(6-Bromohexyl)-1-methyl-2,3-dihydro-1H-indene (3n) was isolated through column chromatography on silica gel with petroleum ether as a colorless oil (39 mg, $67 \%)$. ${ }^{1} \mathrm{H}$ NMR: (400 MHz, Chloroformd) $\delta=7.22-7.07(\mathrm{~m}, 4 \mathrm{H}), 3.42-3.33(\mathrm{~m}, 2 \mathrm{H}), 2.91-2.82(\mathrm{~m}, 2 \mathrm{H}), 2.05-1.95(\mathrm{~m}, 1 \mathrm{H}), 1.88-1.76(\mathrm{~m}, 3 \mathrm{H})$, 1.63-1.38 (m, 4H), 1.34-1.13 (m, 4H), $1.23(\mathrm{~s}, 3 \mathrm{H}) \mathrm{ppm} .{ }^{13} \mathrm{C}$ NMR (101 MHz, Chloroform- $d$ ): $\delta=151.6$, 143.2, 126.2, 126.1, 124.5, 122.6, 47.3, 41.3, 38.6, 34.1, 32.8, 30.3, 29.6, 28.2, 26.8, 24.8 ppm. HRMS (EI): calcd. for $\mathrm{C}_{16} \mathrm{H}_{23} \mathrm{Br}^{+}\left[\mathrm{M}^{+}\right]$: 294.0978, found: 294.0978 .

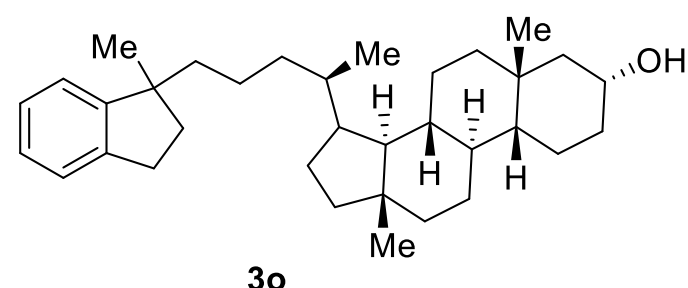

(3R, 5R, 8R,9S, 10R, 13S, 14S)-5,13-Dimethyl-15-((2R)-5-(1-methyl-2,3dihydro-1H-inden-1-yl)pentan-2-yl)hexadecahydro- $1 H$ cyclopenta[a]phenanthren-3-ol (3o) was isolated through column chromatography on silica gel with petroleum ether to the mixture eluent of petroleum ether and ethyl acetate $\quad(10: 1)$ as a yellow oil $(57 \mathrm{mg}, 57 \%) .{ }^{1} \mathrm{H}$ NMR: (400 MHz, Chloroform- $d$ ) $\delta=7.21-7.06(\mathrm{~m}, 4 \mathrm{H}), 3.69-3.54(\mathrm{~m}, 1 \mathrm{H})$, 2.91-2.82 (m, 2H), 2.04-1.93 (m, 2H), 1.87-1.72 (m, 6H), 1.58-1.46 (m, 4H), 1.43-1.31 (m, 10H), 1.28-1.10 (m, 6H), $1.22(\mathrm{~s}$, $3 \mathrm{H}), 0.98-0.78(\mathrm{~m}, 7 \mathrm{H}), 0.91(\mathrm{~s}, 3 \mathrm{H}), 0.66-0.59(\mathrm{~m}, 1 \mathrm{H}), 0.62(\mathrm{~s}, 3 \mathrm{H}) \mathrm{ppm} .{ }^{13} \mathrm{C}$ NMR $(101 \mathrm{MHz}, \mathrm{Chloroform}-d): \delta=151.9$, 143.1, 126.1 (2C), 124.4, 122.6, 71.9, 56.5, 55.8, 47.4, 42.7, 42.1, 41.8, 40.5, 40.2, 38.5, 36.5, 35.9, 35.4, 34.6, 30.6, 30.3, 28.3, 27.2, 26.8, 26.5, 24.3, 23.4, 21.5, 20.9, 18.6, 18.1, 12.1, 10.4 ppm. HRMS (ESI): calcd. for $\mathrm{C}_{34} \mathrm{H}_{53} \mathrm{O}^{+}[\mathrm{M}+\mathrm{H}]^{+}: 477.4091$, found: 477.4095 .

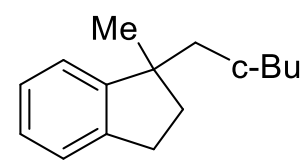

$3 p$

1-(Cyclobutylmethyl)-1-methyl-2,3-dihydro-1H-indene (3p) was isolated through column chromatography on silica gel with petroleum ether to the mixture eluent of petroleum ether and ethyl acetate (20:1) as a colorless oil (21 mg, $53 \%) .{ }^{1} \mathrm{H}$ NMR: (400 MHz, Chloroform- $d$ ) $\delta=7.20-7.06$ (m, $4 \mathrm{H}), 2.90-2.81(\mathrm{~m}, 2 \mathrm{H}), 2.42-2.27(\mathrm{~m}, 1 \mathrm{H}), 2.04-1.95(\mathrm{~m}, 2 \mathrm{H}), 1.93-1.84(\mathrm{~m}, 1 \mathrm{H}), 1.84-1.52(\mathrm{~m}, 7 \mathrm{H})$, 1.19 (s, 3H) ppm. ${ }^{13} \mathrm{C}$ NMR $(101 \mathrm{MHz}$, Chloroform- $d$ ): $\delta=151.9,143.1,126.1,126.0,124.4,122.6$, 


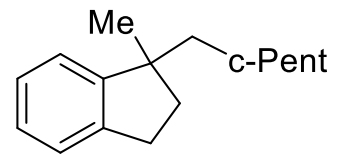

$3 q$

1-(Cyclopentylmethyl)-1-methyl-2,3-dihydro-1H-indene (3q) was isolated through column chromatography on silica gel with petroleum ether to the mixture eluent of petroleum ether and ethyl acetate (20:1) as a colorless oil (22 mg, $51 \%) .{ }^{1} \mathrm{H}$ NMR: (400 MHz, Chloroform- $d$ ) $\delta=7.22-7.07$ $(\mathrm{m}, 4 \mathrm{H}), 2.88(\mathrm{t}, J=7.3 \mathrm{~Hz}, 2 \mathrm{H}), 2.07(\mathrm{dt}, J=12.5,7.1 \mathrm{~Hz}, 1 \mathrm{H}), 1.90-1.70(\mathrm{~m}, 4 \mathrm{H}), 1.66-1.51(\mathrm{~m}$, $4 \mathrm{H}), 1.50-1.37$ (m, 2H), 1.24 (s, 3H), 1.14-0.85 (m, 2H) ppm. ${ }^{13} \mathrm{C}$ NMR (101 MHz, Chloroform- $d$ ): $\delta=152.4,143.1,126.12,126.07,124.4,122.7,47.8,47.7,39.0,37.4,35.1,34.5,30.4,27.5,25.3,24.9$ ppm. HRMS (EI): calcd. for $\mathrm{C}_{16} \mathrm{H}_{22}{ }^{+}\left[\mathrm{M}^{+}\right]:$214.1716, found: 214.1716.

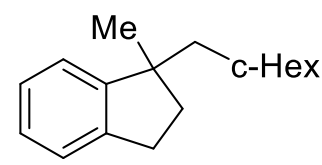

$3 r$

1-(Cyclohexylmethyl)-1-methyl-2,3-dihydro-1H-indene (3r) was isolated through column chromatography on silica gel with petroleum ether as a colorless oil (21 mg, $46 \%) .{ }^{1} \mathrm{H}$ NMR: (400 MHz, Chloroform- $d$ ) $\delta=7.21-7.06(\mathrm{~m}, 4 \mathrm{H}), 2.91-2.83(\mathrm{~m}, 2 \mathrm{H}), 2.09-2.00(\mathrm{~m}, 1 \mathrm{H}), 1.87-1.79(\mathrm{~m}, 1 \mathrm{H})$, 1.69-1.48 (m, 6H), 1.45-1.32 (m, 2H), 1.26-1.05 (m, 3H), $1.22(\mathrm{~s}, 3 \mathrm{H}), 1.01-0.82(\mathrm{~m}, 2 \mathrm{H}) \mathrm{ppm} .{ }^{13} \mathrm{C}$ NMR (101 MHz, Chloroform- $d$ ): $\delta=152.5,142.9,126.1$ (2C), 124.5, 122.5, 48.6, 47.7, 38.8, 36.0, $35.3,34.8,30.3,27.4,26.6(2 \mathrm{C}), 26.4 \mathrm{ppm}$.<smiles>CC(C)CC(C)CC1(C)CCc2ccccc21</smiles>

3s

1-Methyl-1-(2-methylhexyl)-2,3-dihydro-1H-indene (3s) was isolated through column chromatography on silica gel with petroleum ether as a colorless oil (20 mg, 43\%, dr=1:1). ${ }^{1} \mathrm{H}$ NMR: (400 MHz, Chloroform- $d$ ) $\delta=7.21-7.08(\mathrm{~m}, 4 \mathrm{H}), 2.94-2.80(\mathrm{~m}, 2 \mathrm{H}), 2.12-1.98(\mathrm{~m}, 1 \mathrm{H}), 1.89-1.78$ $(\mathrm{m}, 1 \mathrm{H}), 1.72-1.41(\mathrm{~m}, 3 \mathrm{H}), 1.35-1.01(\mathrm{~m}, 7 \mathrm{H}), 1.23(\mathrm{~s}, 3 \mathrm{H}), 0.88-0.76(\mathrm{~m}, 5 \mathrm{H}) \mathrm{ppm} .{ }^{13} \mathrm{C}$ NMR $(101$ MHz, Chloroform- $d$ ): $\delta=152.42$ (152.37), 142.97 (142.93), 126.13 (126.11), 124.5, 122.64 (122.59), 48.4 (48.3), 47.8 (47.7), 39.3 (39.0), 38.9 (38.7), 30.4 (30.3), 29.9 (29.8), 29.4 (29.3), 27.4 (27.3), 23.02 (22.95), 22.6, 21.8, 14.20 (14.17) ppm. Inside the brackets are the chemical shifts of another diastereomer. HRMS (EI): calcd. for $\mathrm{C}_{17} \mathrm{H}_{26}{ }^{+}\left[\mathrm{M}^{+}\right]$: 230.2029, found: 230.2030 .

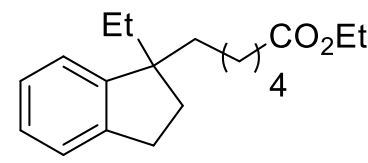

$3 t$

Ethyl 6-(1-ethyl-2,3-dihydro-1H-inden-1-yl)hexanoate (3t) was isolated through column chromatography on silica gel with petroleum ether to the mixture eluent of petroleum ether and ethyl acetate $(20: 1)$ as a colorless oil $(39 \mathrm{mg}, 67 \%) .{ }^{1} \mathrm{H}$ NMR: $(400 \mathrm{MHz}$, Chloroform- $d$ ) $\delta=$ 7.21-7.09 (m, 3H), 7.07-7.01 (m, 1H), $4.10(\mathrm{q}, J=7.1 \mathrm{~Hz}, 2 \mathrm{H}), 2.91-2.80(\mathrm{~m}, 2 \mathrm{H}), 2.24(\mathrm{t}, J=7.5$ $\mathrm{Hz}, 2 \mathrm{H}), 1.96-1.86(\mathrm{~m}, 2 \mathrm{H}), 1.72-1.44(\mathrm{~m}, 6 \mathrm{H}), 1.32-1.08(\mathrm{~m}, 4 \mathrm{H}), 1.24(\mathrm{t}, J=7.1 \mathrm{~Hz}, 3 \mathrm{H}), 0.77$ $(\mathrm{t}, J=7.4 \mathrm{~Hz}, 3 \mathrm{H}) \mathrm{ppm} .{ }^{13} \mathrm{C}$ NMR $(101 \mathrm{MHz}$, Chloroform- $d): \delta=173.9,149.7,143.9,126.2,125.8,124.4,123.5,60.2,51.1$, 38.9, 35.9, 34.4, 31.8, 30.6, 30.0, 25.0, 24.1, 14.3, 8.9 ppm. HRMS (ESI): calcd. for $\mathrm{C}_{19} \mathrm{H}_{29} \mathrm{O}_{2}{ }^{+}[\mathrm{M}+\mathrm{H}]^{+}: 289.2162$, found: 289.2160.

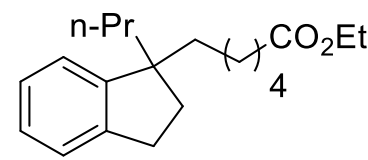

$3 \mathbf{u}$

Ethyl 6-(1-propyl-2,3-dihydro-1H-inden-1-yl)hexanoate (3u) was isolated through column chromatography on silica gel with petroleum ether to the mixture eluent of petroleum ether and ethyl acetate $(20: 1)$ as a colorless oil $(42 \mathrm{mg}, 70 \%) .{ }^{1} \mathrm{H}$ NMR: $(400 \mathrm{MHz}$, Chloroform- $d) \delta=$ 7.21-7.01 (m, 4H), $4.10(\mathrm{q}, J=7.2 \mathrm{~Hz}, 2 \mathrm{H}), 2.91-2.81(\mathrm{~m}, 2 \mathrm{H}), 2.24(\mathrm{t}, J=7.5 \mathrm{~Hz}, 2 \mathrm{H}), 1.96-1.87$ $(\mathrm{m}, 2 \mathrm{H}), 1.68-1.40(\mathrm{~m}, 6 \mathrm{H}), 1.31-1.07(\mathrm{~m}, 6 \mathrm{H}), 1.24(\mathrm{t}, J=7.2 \mathrm{~Hz}, 3 \mathrm{H}), 0.84(\mathrm{t}, J=7.3 \mathrm{~Hz}, 3 \mathrm{H})$ ppm. ${ }^{13} \mathrm{C}$ NMR (101 MHz, Chloroform- $d$ ): $\delta=173.9,150.1,143.8,126.2,125.8,124.4,123.4,60.2,50.9,42.1,39.4,36.4$, 34.4, 30.6, 30.0, 25.0, 24.1, 17.7, 14.9, 14.3 ppm. HRMS (ESI): calcd. for $\mathrm{C}_{20} \mathrm{H}_{30} \mathrm{NaO}_{2}{ }^{+}[\mathrm{M}+\mathrm{Na}]^{+}:$325.2138, found: 325.2139 . 


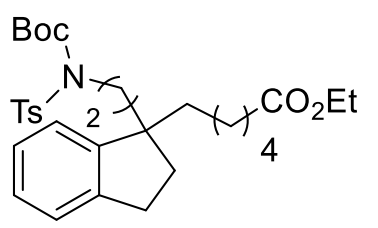

$3 \mathbf{v}$

Ethyl 6-(1-(2-((N-(tert-butoxycarbonyl)-4-methylphenyl)sulfonamido)ethyl)-2,3-dihydro-1Hinden-1-yl)hexanoate (3v) was isolated through column chromatography on silica gel with petroleum ether to the mixture eluent of petroleum ether and ethyl acetate (10:1) as a yellow oil (62 mg, $56 \%$ ). ${ }^{1} \mathrm{H}$ NMR: (400 MHz, Chloroform- $d$ ) $\delta=7.76-7.70$ (m, 2H), 7.30-7.24 (m, 2H), 7.21-7.12 (m, 4H), $4.10(\mathrm{q}, J=7.1 \mathrm{~Hz}, 2 \mathrm{H}), 3.82-3.58(\mathrm{~m}, 2 \mathrm{H}), 2.96-2.87(\mathrm{~m}, 2 \mathrm{H}), 2.42(\mathrm{~s}, 3 \mathrm{H})$, 2.29-2.21 (m, 2H), 2.17-1.95 (m, 4H), 1.69-1.49 (m, 4H), 1.35-1.19 (m, 4H), $1.30(\mathrm{~s}, 9 \mathrm{H}), 1.23(\mathrm{t}$, $J=7.2 \mathrm{~Hz}, 3 \mathrm{H}) \mathrm{ppm} .{ }^{13} \mathrm{C}$ NMR (101 MHz, Chloroform- $d$ ): $\delta=173.8,150.8,148.4,144.0,143.5,137.5,129.2$ (2C), 127.8 (2C), 126.6, 126.2, 124.6, 123.5, 83.9, 60.2, 49.9, 44.2, 40.0, 39.2, 35.6, 34.4, 30.6, 29.8, 27.9 (3C), 24.9, 24.0, 21.6, 14.3 ppm. HRMS (ESI): calcd. for $\mathrm{C}_{31} \mathrm{H}_{44} \mathrm{NO}_{6} \mathrm{~S}^{+}[\mathrm{M}+\mathrm{H}]^{+}$: 558.2884, found: 558.2887.

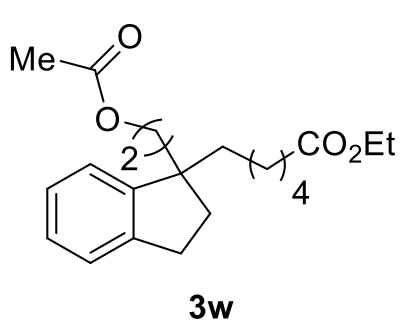

Ethyl 6-(1-(2-acetoxyethyl)-2,3-dihydro-1H-inden-1-yl)hexanoate (3w) was isolated through column chromatography on silica gel with petroleum ether to the mixture eluent of petroleum ether and ethyl acetate (20:1) as a yellow oil (43 mg, $62 \%) .{ }^{1} \mathrm{H}$ NMR: (400 MHz, Chloroformd) $\delta=7.21-7.11(\mathrm{~m}, 3 \mathrm{H}), 7.10-7.04(\mathrm{~m}, 1 \mathrm{H}), 4.17-3.93(\mathrm{~m}, 4 \mathrm{H}), 2.93-2.85(\mathrm{~m}, 2 \mathrm{H}), 2.30-2.19$ (m, 2H), 2.06-1.85 (m, 4H), $1.98(\mathrm{~s}, 3 \mathrm{H}), 1.69-1.44(\mathrm{~m}, 4 \mathrm{H}), 1.33-1.11(\mathrm{~m}, 4 \mathrm{H}), 1.24(\mathrm{t}, J=7.1$ $\mathrm{Hz}, 3 \mathrm{H}) \mathrm{ppm} .{ }^{13} \mathrm{C}$ NMR (101 MHz, Chloroform- $d$ ): $\delta=173.8,171.1,148.6,143.5,126.6,126.1$, $124.7,123.3,62.0,60.2,49.5,39.7,37.4,36.4,34.3,30.5,29.8,24.9,24.1,21.0,14.3$ ppm. HRMS (ESI): calcd. for $\mathrm{C}_{21} \mathrm{H}_{31} \mathrm{O}_{4}{ }^{+}[\mathrm{M}+\mathrm{H}]^{+}: 347.2217$, found: 347.2219 .

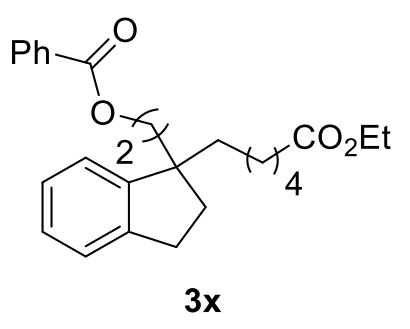

2-(1-(6-Ethoxy-6-oxohexyl)-2,3-dihydro-1H-inden-1-yl)ethyl benzoate $\mathbf{( 3 \mathbf { x } )}$ was isolated through column chromatography on silica gel with petroleum ether to the mixture eluent of petroleum ether and ethyl acetate (20:1) as a yellow oil (52 mg, $64 \%) .{ }^{1} \mathrm{H}$ NMR: (400 MHz, Chloroform- $d$ ) $\delta=8.00-7.92(\mathrm{~m}, 2 \mathrm{H}), 7.57-7.51(\mathrm{~m}, 1 \mathrm{H}), 7.45-7.37(\mathrm{~m}, 2 \mathrm{H}), 7.22-7.09(\mathrm{~m}, 4 \mathrm{H})$, 4.37-4.18 (m, 2H), 4.10 (q, J=7.2 Hz, 2H), 3.00-2.83 (m, 2H), 2.34-2.20 (m, 3H), 2.17-1.98 (m, $3 \mathrm{H}), 1.77-1.51(\mathrm{~m}, 4 \mathrm{H}), 1.39-1.12(\mathrm{~m}, 4 \mathrm{H}), 1.23$ (t, $J=7.3 \mathrm{~Hz}, 3 \mathrm{H}) \mathrm{ppm} .{ }^{13} \mathrm{C}$ NMR (101 MHz, Chloroform- $d$ ): $\delta=173.8,166.6,148.7,143.5,132.8,130.4,129.5$ (2C), 128.3, 126.7, 126.2, 124.7, 123.3, 62.6, 60.2, 49.7, 39.8, 37.5, 36.6, 34.3, 30.6, 29.8, 29.1, 24.9, 24.1, 14.3 ppm. HRMS (ESI): calcd. for $\mathrm{C}_{26} \mathrm{H}_{33} \mathrm{O}_{4}{ }^{+}$ $[\mathrm{M}+\mathrm{H}]^{+}:$409.2373, found: 409.2373 .

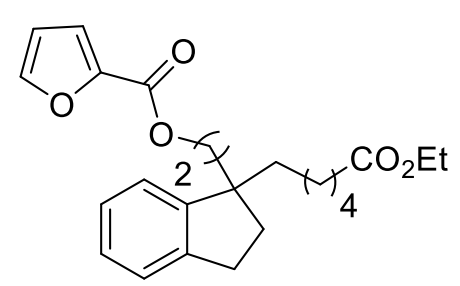

3y

2-(1-(6-Ethoxy-6-oxohexyl)-2,3-dihydro-1H-inden-1-yl)ethyl furan-2-carboxylate (3y) was isolated through column chromatography on silica gel (petroleum ether: EtOAc $=10: 1$ ) as a colorless oil (54 mg, $68 \%) .{ }^{1} \mathrm{H}$ NMR: $(400 \mathrm{MHz}$, Chloroform- $d$ ) $\delta=7.59-7.52(\mathrm{~m}, 1 \mathrm{H}), 7.23-$ $7.06(\mathrm{~m}, 5 \mathrm{H}), 6.52-6.45(\mathrm{~m}, 1 \mathrm{H}), 4.34-4.17(\mathrm{~m}, 2 \mathrm{H}), 4.10(\mathrm{q}, J=7.1 \mathrm{~Hz}, 2 \mathrm{H}), 2.95-2.86(\mathrm{~m}$, $2 \mathrm{H}), 2.28-2.19$ (m, 2H), 2.17-1.96 (m, 4H), 1.75-1.50 (m, 4H), 1.35-1.15 (m, 4H), 1.23 (t, $J$ $=7.2 \mathrm{~Hz}, 3 \mathrm{H}) \mathrm{ppm} .{ }^{13} \mathrm{C}$ NMR $(101 \mathrm{MHz}$, Chloroform-d): $\delta=173.7,158.7,148.5,146.2,144.8$, 143.5, 126.7, 126.2, 124.7, 123.3, 117.7, 111.8, 62.6, 60.2, 49.6, 39.8, 37.4, 36.5, 34.3, 30.5, 29.8, 24.9, 24.1, 14.3 ppm. HRMS (ESI): calcd. for $\mathrm{C}_{24} \mathrm{H}_{31} \mathrm{O}_{5}^{+}[\mathrm{M}+\mathrm{H}]^{+}:$399.2166, found: 399.2167.

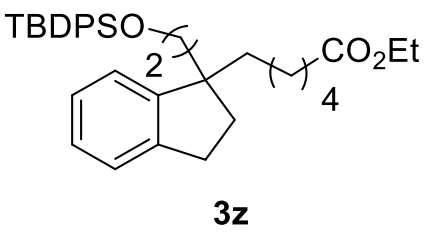

Ethyl 6-(1-(2-((tert-butyldiphenylsilyl)oxy)ethyl)-2,3-dihydro-1H-inden-1-yl)hexanoate (3z) was isolated through column chromatography on silica gel with petroleum ether as a colorless oil (64 mg, $59 \%)$. ${ }^{1} \mathrm{H}$ NMR: (400 MHz, Chloroform- $d$ ) $\delta=7.67-7.57$ (m, 4H), 7.45-7.31 (m, $6 \mathrm{H}), 7.17-7.04(\mathrm{~m}, 3 \mathrm{H}), 6.95-6.88(\mathrm{~m}, 1 \mathrm{H}), 4.10(\mathrm{q}, J=7.1 \mathrm{~Hz}, 2 \mathrm{H}), 3.71-3.53(\mathrm{~m}, 2 \mathrm{H}), 2.86-$ $2.73(\mathrm{~m}, 2 \mathrm{H}), 2.27-2.15(\mathrm{~m}, 2 \mathrm{H}), 2.00-1.76(\mathrm{~m}, 4 \mathrm{H}), 1.59-1.36(\mathrm{~m}, 4 \mathrm{H}), 1.27-1.06(\mathrm{~m}, 4 \mathrm{H})$, $1.23(\mathrm{t}, J=7.1 \mathrm{~Hz}, 3 \mathrm{H}), 1.01(\mathrm{~s}, 9 \mathrm{H}) \mathrm{ppm} .{ }^{13} \mathrm{C}$ NMR (101 MHz, Chloroform- $d$ ): $\delta=173.8,149.3,143.5,135.61(2 \mathrm{C}), 135.57$ (2C), 134.0, 133.9, 129.6, 129.5, 127.6 (4C), 126.3, 125.9, 124.5, 123.4, 61.2, 60.2, 49.6 41.4, 39.6, 36.6, 34.4, 30.5, 29.9, 26.9 (3C), 25.0, 24.1, 19.1, 14.3 ppm. HRMS (ESI): calcd. for $\mathrm{C}_{35} \mathrm{H}_{47} \mathrm{O}_{3} \mathrm{Si}^{+}[\mathrm{M}+\mathrm{H}]^{+}:$543.3289, found: 543.3292 . 


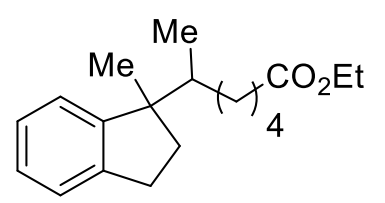

3aa

Ethyl 6-(1-methyl-2,3-dihydro-1H-inden-1-yl)heptanoate (3aa) was isolated through column chromatography on silica gel with petroleum ether to the mixture eluent of petroleum ether and ethyl acetate (20:1) as a colorless oil (32 mg, $55 \%)$. ${ }^{1} \mathrm{H}$ NMR: (400 MHz, Chloroform-d) $\delta=$ 7.20-7.05 (m, 4H), $4.11(\mathrm{q}, J=7.1 \mathrm{~Hz}, 2 \mathrm{H}), 2.91-2.82(\mathrm{~m}, 2 \mathrm{H}), 2.30-2.21(\mathrm{~m}, 2 \mathrm{H}), 2.05-1.95(\mathrm{~m}$, $1 \mathrm{H}), 1.85-1.75(\mathrm{~m}, 1 \mathrm{H}), 1.69-1.39(\mathrm{~m}, 4 \mathrm{H}), 1.37-1.08(\mathrm{~m}, 6 \mathrm{H}), 1.24(\mathrm{t}, J=7.1 \mathrm{~Hz}, 3 \mathrm{H}), 1.22(\mathrm{~s}$, $3 \mathrm{H}) \mathrm{ppm} .{ }^{13} \mathrm{C}$ NMR $(101 \mathrm{MHz}$, Chloroform-d): $\delta=173.9,151.7,143.2,126.2,126.1,124.5,122.6$, $60.2,47.3,41.4,38.6,34.4,30.3,30.1,29.2,26.8,25.0,24.8,14.3$ ppm. HRMS (ESI): calcd. for $\mathrm{C}_{19} \mathrm{H}_{29} \mathrm{O}_{2}{ }^{+}[\mathrm{M}+\mathrm{H}]^{+}: 289.2162$, found: 289.2164 .

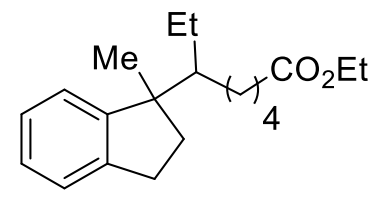

$3 a b$

Ethyl 6-(1-methyl-2,3-dihydro-1H-inden-1-yl)octanoate (3ab) was isolated through column chromatography on silica gel to the mixture eluent of petroleum ether and ethyl acetate $\quad(50: 1)$ as a colorless oil (31 mg, $51 \%)$. ${ }^{1} \mathrm{H}$ NMR: (400 MHz, Chloroform- $d$ ) $\delta=7.21-7.07$ (m, 4H), 4.11 (q, $J=7.1 \mathrm{~Hz}, 2 \mathrm{H}), 2.92-2.82(\mathrm{~m}, 2 \mathrm{H}), 2.31-2.22(\mathrm{~m}, 2 \mathrm{H}), 2.05-1.94(\mathrm{~m}, 1 \mathrm{H}), 1.86-1.74(\mathrm{~m}, 1 \mathrm{H}), 1.65-$ $1.53(\mathrm{~m}, 4 \mathrm{H}), 1.31-1.18(\mathrm{~m}, 8 \mathrm{H}), 1.24(\mathrm{t}, J=7.1 \mathrm{~Hz}, 3 \mathrm{H}), 1.22(\mathrm{~s}, 3 \mathrm{H}) \mathrm{ppm} .{ }^{13} \mathrm{C}$ NMR $(101 \mathrm{MHz}$, Chloroform- $d$ ): $\delta=173.9,151.7,143.2,126.13,126.09,124.5,122.6,60.2,47.3,41.4,38.6,34.4$, 30.3, 30.2, 29.3, 29.2, 26.8, 25.0, 24.9, 14.3 ppm. HRMS (ESI): calcd. for $\mathrm{C}_{20} \mathrm{H}_{31} \mathrm{O}_{2}{ }^{+}[\mathrm{M}+\mathrm{H}]^{+}:$303.2319, found: 303.2323 .

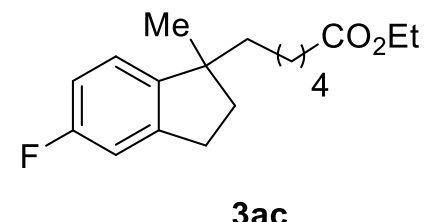

3ac

Ethyl 6-(5-fluoro-1-methyl-2,3-dihydro-1H-inden-1-yl)hexanoate (3ac) was isolated through column chromatography on silica gel with petroleum ether to the mixture eluent of petroleum ether and ethyl acetate (20:1) as a colorless oil (37 mg, $64 \%) .{ }^{1} \mathrm{H}$ NMR: (400 MHz, Chloroform-d) $\delta=7.02-6.97(\mathrm{~m}, 1 \mathrm{H}), 6.88-6.79(\mathrm{~m}, 2 \mathrm{H}), 4.11(\mathrm{q}, J=7.1 \mathrm{~Hz}, 2 \mathrm{H}), 2.88-2.79$ $(\mathrm{m}, 2 \mathrm{H}), 2.29-2.21(\mathrm{~m}, 2 \mathrm{H}), 2.07-1.96(\mathrm{~m}, 1 \mathrm{H}), 1.88-1.78(\mathrm{~m}, 1 \mathrm{H}), 1.65-1.40(\mathrm{~m}, 4 \mathrm{H}), 1.32-$ $1.12(\mathrm{~m}, 4 \mathrm{H}), 1.24(\mathrm{t}, J=7.1 \mathrm{~Hz}, 3 \mathrm{H}), 1.20(\mathrm{~s}, 3 \mathrm{H}) \mathrm{ppm} .{ }^{13} \mathrm{C}$ NMR $(101 \mathrm{MHz}$, Chloroform-d): $\delta=173.8,161.9$ (d, $J=242.3$ $\mathrm{Hz}), 147.0(\mathrm{~d}, J=2.4 \mathrm{~Hz}), 145.2(\mathrm{~d}, J=7.9 \mathrm{~Hz}), 123.4(\mathrm{~d}, J=8.8 \mathrm{~Hz}), 112.9(\mathrm{~d}, J=22.3 \mathrm{~Hz}), 111.3(\mathrm{~d}, J=21.6 \mathrm{~Hz}), 60.2$, $46.7,41.3,38.9,34.4,30.3,29.9,27.0,24.9,24.6,14.3$ ppm. ${ }^{19} \mathrm{~F}$ NMR $\left(376 \mathrm{MHz}, \mathrm{CDCl}_{3}\right) \delta=-117.96--118.13(\mathrm{~m}, 1 \mathrm{~F})$ ppm. HRMS (ESI): calcd. for $\mathrm{C}_{18} \mathrm{H}_{26} \mathrm{FO}_{2}{ }^{+}[\mathrm{M}+\mathrm{H}]^{+}: 293.1911$, found: 293.1914 .

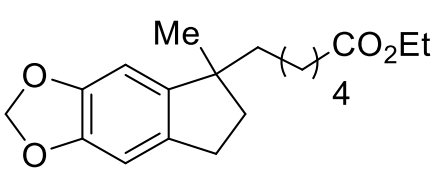

3ad

Ethyl 6-(5-methyl-6,7-dihydro-5H-indeno[5,6-d][1,3]dioxol-5-yl)hexanoate (3ad) was isolated through column chromatography on silica gel with petroleum ether to the mixture eluent of petroleum ether and ethyl acetate $\quad(20: 1)$ as a colorless oil (55 mg, $60 \%) .{ }^{1} \mathrm{H}$ NMR: $(400 \mathrm{MHz}$, Chloroform- $d$ ) $\delta=6.64(\mathrm{~s}, 1 \mathrm{H}), 6.55(\mathrm{~s}, 1 \mathrm{H}), 5.92-5.88(\mathrm{~m}, 2 \mathrm{H}), 4.11(\mathrm{q}, J=7.1$ $\mathrm{Hz}, 2 \mathrm{H}), 2.79-2.71(\mathrm{~m}, 2 \mathrm{H}), 2.30-2.21(\mathrm{~m}, 2 \mathrm{H}), 2.05-1.95(\mathrm{~m}, 1 \mathrm{H}), 1.86-1.76(\mathrm{~m}, 1 \mathrm{H}), 1.64-$ $1.55(\mathrm{~m}, 2 \mathrm{H}), 1.54-1.39(\mathrm{~m}, 2 \mathrm{H}), 1.35-1.20(\mathrm{~m}, 4 \mathrm{H}), 1.24(\mathrm{t}, J=7.1 \mathrm{~Hz}, 3 \mathrm{H}), 1.17(\mathrm{~s}, 3 \mathrm{H}) \mathrm{ppm} .{ }^{13} \mathrm{C}$ NMR $(101 \mathrm{MHz}$, Chloroform- $d$ ): $\delta=173.9,146.3,146.2,144.5,135.6,105.0,103.3,100.8,60.2,47.1,41.4,39.0,34.4,30.2,29.9,27.1,25.0$, 24.6, 14.3 ppm. HRMS (ESI): calcd. for $\mathrm{C}_{19} \mathrm{H}_{27} \mathrm{O}_{4}{ }^{+}[\mathrm{M}+\mathrm{H}]^{+}$: $\quad 319.1904$, found: 319.1901 .

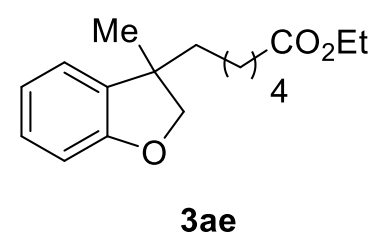

Ethyl 6-(3-methyl-2,3-dihydrobenzofuran-3-yl)hexanoate (3ae) was isolated through column chromatography on silica gel with petroleum ether to the mixture eluent of petroleum ether and ethyl acetate (20:1) as a yellow oil (26 mg, $47 \%)$. ${ }^{1} \mathrm{H}$ NMR: $(400 \mathrm{MHz}$, Chloroform- $d) \delta=7.15$ 7.09 (m, 1H), 7.08-7.03 (m, 1H), 6.91-6.83 (m, 1H), 6.81-6.75 (m, 1H), 4.34 (d, J = 8.6 Hz, 1H), $4.15(\mathrm{~d}, J=8.6 \mathrm{~Hz}, 1 \mathrm{H}), 4.11(\mathrm{q}, J=7.1 \mathrm{~Hz}, 2 \mathrm{H}), 2.30-2.19(\mathrm{~m}, 2 \mathrm{H}), 1.64-1.54(\mathrm{~m}, 4 \mathrm{H}), 1.33(\mathrm{~s}$, $3 \mathrm{H}), 1.30-1.05(\mathrm{~m}, 4 \mathrm{H}), 1.24(\mathrm{t}, J=7.1 \mathrm{~Hz}, 3 \mathrm{H}) \mathrm{ppm} .{ }^{13} \mathrm{C}$ NMR $(101 \mathrm{MHz}$, Chloroform- $d): \delta=173.7,159.5,135.2,128.0$, 122.8, 120.4, 109.5, 82.5, 60.2, 45.2, 40.8, 34.3, 29.6, 25.6, 24.8, 24.3, 14.3 ppm. HRMS (ESI): calcd. for $\mathrm{C}_{17} \mathrm{H}_{25} \mathrm{O}_{3}{ }^{+}\left[\mathrm{M}+\mathrm{H}^{+}\right.$: 277.1798, found: 277.1797. 


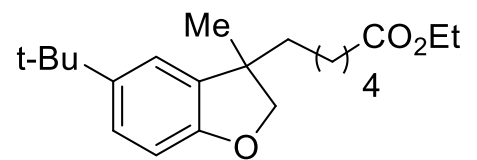

3af

Ethyl 6-(5-(tert-butyl)-3-methyl-2,3-dihydrobenzofuran-3-yl)hexanoate (3af) was isolated through column chromatography on silica gel with petroleum ether to the mixture eluent of petroleum ether and ethyl acetate (20:1) as a yellow oil (27 mg, $41 \%) .{ }^{1} \mathrm{H}$ NMR: (400 MHz, Chloroform- $d$ ) $\delta=7.17-7.11(\mathrm{~m}, 1 \mathrm{H}), 7.09-7.04(\mathrm{~m}, 1 \mathrm{H}), 6.74-6.66(\mathrm{~m}, 1 \mathrm{H}), 4.37-$ $4.26(\mathrm{~m}, 1 \mathrm{H}), 4.19-4.05(\mathrm{~m}, 3 \mathrm{H}), 2.29-2.20(\mathrm{~m}, 2 \mathrm{H}), 1.65-1.55(\mathrm{~m}, 4 \mathrm{H}), 1.35-1.28(\mathrm{~m}$, $3 \mathrm{H}), 1.32(\mathrm{~s}, 3 \mathrm{H}), 1.30(\mathrm{~s}, 9 \mathrm{H}), 1.24(\mathrm{t}, \mathrm{J}=7.1 \mathrm{~Hz}, 3 \mathrm{H}), 1.18-1.08(\mathrm{~m}, 1 \mathrm{H}) \mathrm{ppm} .{ }^{13} \mathrm{C}$ NMR $(101 \mathrm{MHz}$, Chloroform- $d$ ): $\delta=$ 173.8, 157.3, 143.5, 134.9, 124.7, 119.6, 108.6, 82.8, 60.2, 45.4, 40.7, 34.4, 34.3, 31.8(3C), 29.6, 25.3, 24.8, 24.3, 14.3 ppm. HRMS (ESI): calcd. for $\mathrm{C}_{21} \mathrm{H}_{32} \mathrm{NaO}_{3}{ }^{+}[\mathrm{M}+\mathrm{Na}]^{+}: 355.2244$, found: 355.2247 .

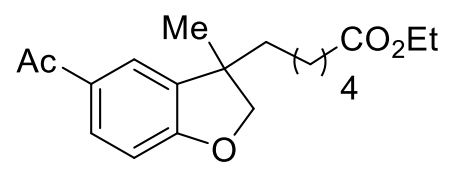

3ag

Ethyl 6-(5-acetyl-3-methyl-2,3-dihydrobenzofuran-3-yl)hexanoate (3ag) was isolated through column chromatography on silica gel with petroleum ether to the mixture eluent of petroleum ether and ethyl acetate (10:1) as a yellow oil (27 mg, $42 \%) .{ }^{1} \mathrm{H}$ NMR: (400 MHz, Chloroform- $d$ ) $\delta=7.80(\mathrm{dd}, \mathrm{J}=8.4,1.9 \mathrm{~Hz}, 1 \mathrm{H}), 7.73(\mathrm{~d}, \mathrm{~J}=1.9 \mathrm{~Hz}, 1 \mathrm{H}), 6.79(\mathrm{~d}, \mathrm{~J}=8.3 \mathrm{~Hz}$, $1 \mathrm{H}), 4.44(\mathrm{~d}, \mathrm{~J}=8.9 \mathrm{~Hz}, 1 \mathrm{H}), 4.24(\mathrm{~d}, \mathrm{~J}=8.8 \mathrm{~Hz}, 1 \mathrm{H}), 4.11(\mathrm{q}, \mathrm{J}=7.1 \mathrm{~Hz}, 2 \mathrm{H}), 2.56(\mathrm{~s}, 3 \mathrm{H})$, $2.28-2.21(\mathrm{~m}, 2 \mathrm{H}), 1.68-1.53(\mathrm{~m}, 4 \mathrm{H}), 1.36(\mathrm{~s}, 3 \mathrm{H}), 1.32-1.19(\mathrm{~m}, 7 \mathrm{H})$ ppm. ${ }^{13} \mathrm{C}$ NMR $(101 \mathrm{MHz}$, Chloroform- $d): \delta=$ 196.8, 173.7, 164.0, 136.1, 130.8, 130.6, 123.4, 109.1, 83.5, 60.2, 44.8, 40.8, 34.2, 29.5 26.5, 25.8, 24.8, 24.2, 14.3 ppm. HRMS (ESI): calcd. for $\mathrm{C}_{19} \mathrm{H}_{26} \mathrm{NaO}_{4}{ }^{+}[\mathrm{M}+\mathrm{Na}]^{+}: 341.1723$, found: 341.1724 .

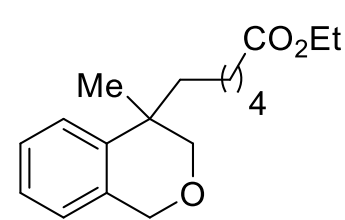

$3 a h$

Ethyl 6-(4-methylisochroman-4-yl)hexanoate (3ah) was isolated through column chromatography on silica gel with petroleum ether to the mixture eluent of petroleum ether and ethyl acetate (20:1) as a yellow oil (41 mg, $70 \%) .{ }^{1} \mathrm{H}$ NMR: (400 MHz, Chloroform- $d$ ) $\delta=7.28-7.25(\mathrm{~m}, 1 \mathrm{H}), 7.23-7.17$ $(\mathrm{m}, 1 \mathrm{H}), 7.17-7.10(\mathrm{~m}, 1 \mathrm{H}), 6.98-6.92(\mathrm{~m}, 1 \mathrm{H}), 4.77(\mathrm{~s}, 2 \mathrm{H}), 4.10(\mathrm{q}, J=7.2 \mathrm{~Hz}, 2 \mathrm{H}), 3.79(\mathrm{~d}, J=$ $11.3 \mathrm{~Hz}, 1 \mathrm{H}), 3.54(\mathrm{~d}, J=11.3 \mathrm{~Hz}, 1 \mathrm{H}), 2.32-2.19(\mathrm{~m}, 2 \mathrm{H}), 1.65-1.54(\mathrm{~m}, 4 \mathrm{H}), 1.34-1.16(\mathrm{~m}, 4 \mathrm{H})$, $1.24(\mathrm{t}, J=7.1 \mathrm{~Hz}, 3 \mathrm{H}), 1.21(\mathrm{~s}, 3 \mathrm{H}) \mathrm{ppm} .{ }^{13} \mathrm{C}$ NMR $(101 \mathrm{MHz}$, Chloroform- $d$ ): $\delta=173.8,142.0$, 133.9, 126.6, 126.0, 125.7, 124.0, 74.7, 68.9, 60.2, 40.7, 36.2, 34.4, 29.9, 29.1, 24.9, 24.1, 14.3 ppm. HRMS (ESI): calcd. for $\mathrm{C}_{18} \mathrm{H}_{27} \mathrm{O}_{3}{ }^{+}[\mathrm{M}+\mathrm{H}]^{+}:$291.1955, found: 291.1956 .

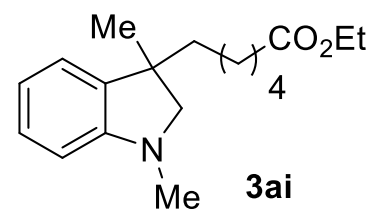

Ethyl 6-(1,3-dimethylindolin-3-yl)hexanoate (3ai) was isolated through column chromatography on silica gel with petroleum ether to the mixture eluent of petroleum ether and ethyl acetate $\quad(20: 1)$ as a yellow oil (45 mg, $78 \%$ ). ${ }^{1} \mathrm{H}$ NMR: (400 MHz, Chloroform- $d$ ) $\delta=7.12-7.05(\mathrm{~m}, 1 \mathrm{H}), 6.98$ $6.93(\mathrm{~m}, 1 \mathrm{H}), 6.72-6.65(\mathrm{~m}, 1 \mathrm{H}), 6.50-6.43(\mathrm{~m}, 1 \mathrm{H}), 4.11(\mathrm{q}, J=7.1 \mathrm{~Hz}, 2 \mathrm{H}), 3.17(\mathrm{~d}, J=8.6 \mathrm{~Hz}$, $1 \mathrm{H}), 2.97(\mathrm{~d}, J=8.6 \mathrm{~Hz}, 1 \mathrm{H}), 2.73(\mathrm{~s}, 3 \mathrm{H}), 2.31-2.21(\mathrm{~m}, 2 \mathrm{H}), 1.66-1.52(\mathrm{~m}, 3 \mathrm{H}), 1.37-1.15(\mathrm{~m}$, $5 \mathrm{H}), 1.24(\mathrm{t}, J=7.1 \mathrm{~Hz}, 3 \mathrm{H}), 1.22(\mathrm{~s}, 3 \mathrm{H}) \mathrm{ppm} .{ }^{13} \mathrm{C}$ NMR $(101 \mathrm{MHz}$, Chloroform- $d): \delta=173.8,152.3,138.2,127.5,122.2$, 117.6, 107.2, 68.3, 60.2, 43.6, 40.2, 36.0, 34.4, 29.8, 25.4, 24.9, 24.5, 14.3 ppm. HRMS (ESI): calcd. for $\mathrm{C}_{18} \mathrm{H}_{28} \mathrm{NO}_{2}{ }^{+}[\mathrm{M}+\mathrm{H}]^{+}$: 290.2115, found: 290.2113 .

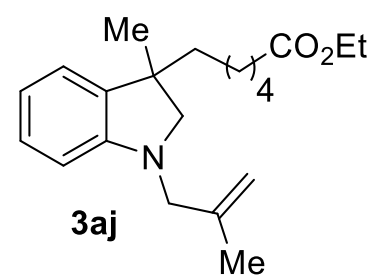

Ethyl 6-(1-methyl-3-(2-methylallyl)indolin-3-yl)hexanoate (3aj) was isolated through column chromatography on silica gel with petroleum ether to the mixture eluent of petroleum ether and ethyl acetate (20:1) as a yellow oil (49 mg, $75 \%)$. ${ }^{1} \mathrm{H}$ NMR: (400 MHz, Chloroform- $d$ ) $\delta=7.05$ $(\mathrm{td}, J=7.7,1.3 \mathrm{~Hz}, 1 \mathrm{H}), 6.95(\mathrm{dd}, J=7.2,1.3 \mathrm{~Hz}, 1 \mathrm{H}), 6.65(\mathrm{td}, J=7.4,1.0 \mathrm{~Hz}, 1 \mathrm{H}), 6.44(\mathrm{~d}, J=$ $7.8 \mathrm{~Hz}, 1 \mathrm{H}), 4.97-4.82(\mathrm{~m}, 2 \mathrm{H}), 4.11(\mathrm{q}, J=7.1 \mathrm{~Hz}, 2 \mathrm{H}), 3.65-3.46(\mathrm{~m}, 2 \mathrm{H}), 3.18(\mathrm{~d}, J=8.8 \mathrm{~Hz}$, $1 \mathrm{H}), 2.99(\mathrm{~d}, J=8.8 \mathrm{~Hz}, 1 \mathrm{H}), 2.31-2.19(\mathrm{~m}, 2 \mathrm{H}), 1.76(\mathrm{~s}, 3 \mathrm{H}), 1.66-1.50(\mathrm{~m}, 4 \mathrm{H}), 1.43-1.13(\mathrm{~m}$, 4H), $1.24(\mathrm{t}, J=7.1 \mathrm{~Hz}, 3 \mathrm{H}), 1.27$ (s, 3H) ppm. ${ }^{13} \mathrm{C}$ NMR (101 MHz, Chloroform- $d$ ): $\delta=173.8,151.6,142.4,137.6,127.4$, 122.3, 117.1, 112.0, 106.6, 65.7, 60.2, 55.4, 43.4, 40.6, 34.4, 29.8, 26.0, 24.9, 24.5, 20.4, 14.3 ppm. HRMS (ESI): calcd. for $\mathrm{C}_{21} \mathrm{H}_{32} \mathrm{NO}_{2}{ }^{+}[\mathrm{M}+\mathrm{H}]^{+}: 330.2428$, found: 330.2425 . 
<smiles>CCOC(=O)CC1(C)CN(Cc2ccccc2)Cc2ccccc21</smiles>

3ak

Ethyl 6-(2-benzyl-4-methyl-1,2,3,4-tetrahydroisoquinolin-4-yl)hexanoate (3ak) was isolated through column chromatography on silica gel (petroleum ether: EtOAc= 20:1) as a yellow oil (46 mg, $61 \%$ ). ${ }^{1} \mathrm{H}$ NMR: (400 MHz, Chloroform- $d$ ) $\delta=7.39(\mathrm{~d}, J=7.5 \mathrm{~Hz}, 2 \mathrm{H}), 7.32(\mathrm{t}, J=7.4 \mathrm{~Hz}, 2 \mathrm{H})$, 7.30-7.20 (m, 2H), 7.16 (t, $J=7.5 \mathrm{~Hz}, 1 \mathrm{H}), 7.08(\mathrm{t}, J=7.4 \mathrm{~Hz}, 1 \mathrm{H}), 6.97$ (d, $J=7.6 \mathrm{~Hz}, 1 \mathrm{H}), 4.11$ (q, $J=7.1 \mathrm{~Hz}, 2 \mathrm{H}), 3.71-3.52(\mathrm{~m}, 4 \mathrm{H}), 2.58(\mathrm{~d}, J=11.3 \mathrm{~Hz}, 1 \mathrm{H}), 2.27(\mathrm{~d}, J=11.3 \mathrm{~Hz}, 1 \mathrm{H}), 2.23(\mathrm{t}$, $J=7.6 \mathrm{~Hz}, 2 \mathrm{H}), 1.82-1.70(\mathrm{~m}, 1 \mathrm{H}), 1.67-1.44(\mathrm{~m}, 3 \mathrm{H}), 1.35-1.13(\mathrm{~m}, 4 \mathrm{H}), 1.24(\mathrm{t}, J=7.1 \mathrm{~Hz}, 3 \mathrm{H})$, $1.21(\mathrm{~s}, 3 \mathrm{H}) \mathrm{ppm} .{ }^{13} \mathrm{C}$ NMR (101 MHz, Chloroform- $d$ ): $\delta=173.9,143.6,138.9,134.5,129.0(2 \mathrm{C}), 128.2(2 \mathrm{C}), 127.0,126.31$, 126.27, 126.2, 125.3, 63.0, 61.3, 60.2, 57.9, 41.9, 38.2, 34.4, 29.9, 27.2, 24.9, 24.0, 14.3 ppm. HRMS (ESI): calcd. for $\mathrm{C}_{25} \mathrm{H}_{34} \mathrm{NO}_{2}{ }^{+}[\mathrm{M}+\mathrm{H}]^{+}: 380.2584$, found: 380.2585 .

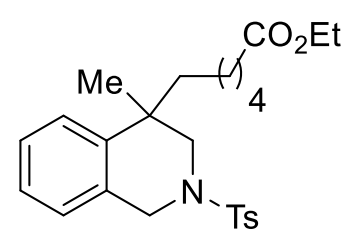

3al

Ethyl 6-(4-methyl-2-tosyl-1,2,3,4-tetrahydroisoquinolin-4-yl)hexanoate (3al) were isolated through column chromatography on silica gel with petroleum ether to the mixture eluent of petroleum ether and ethyl acetate (20:1) as a yellow oil (57 mg, $64 \%) .{ }^{1} \mathrm{H}$ NMR: $(400 \mathrm{MHz}$, Chloroform- $d$ ) $\delta=7.74$ $(\mathrm{d}, J=8.3 \mathrm{~Hz}, 2 \mathrm{H}), 7.35(\mathrm{~d}, J=8.1 \mathrm{~Hz}, 2 \mathrm{H}), 7.26-7.07(\mathrm{~m}, 3 \mathrm{H}), 6.99(\mathrm{dd}, J=7.6,1.4 \mathrm{~Hz}, 1 \mathrm{H}), 4.30$ (d, $J=14.7 \mathrm{~Hz}, 1 \mathrm{H}), 4.16-4.02(\mathrm{~m}, 3 \mathrm{H}), 3.29(\mathrm{~d}, J=11.5 \mathrm{~Hz}, 1 \mathrm{H}), 2.77$ (d, $J=11.6 \mathrm{~Hz}, 1 \mathrm{H}), 2.43$ (s, 3H), $2.25(\mathrm{t}, J=7.5 \mathrm{~Hz}, 2 \mathrm{H}), 1.82-1.68(\mathrm{~m}, 1 \mathrm{H}), 1.65-1.52(\mathrm{~m}, 3 \mathrm{H}), 1.32-1.17(\mathrm{~m}, 4 \mathrm{H}), 1.24(\mathrm{t}, J$ $=7.1 \mathrm{~Hz}, 3 \mathrm{H}), 1.22(\mathrm{~s}, 3 \mathrm{H}) \mathrm{ppm} .{ }^{13} \mathrm{C}$ NMR $(101 \mathrm{MHz}$, Chloroform- $d$ ): $\delta=173.8,143.7,142.0,132.9,130.7,129.8(2 \mathrm{C})$, 127.8 (2C), 126.9, 126.3, 126.12, 126.07, 60.2, 53.3, 48.4, 40.9, 38.1, 34.3, 29.7, 25.8, 24.9, 23.8, 21.6, 14.3 ppm. HRMS (ESI): calcd. for $\mathrm{C}_{25} \mathrm{H}_{34} \mathrm{NO}_{4} \mathrm{~S}^{+}[\mathrm{M}+\mathrm{H}]^{+}$: 444.2203 , found: 444.2202 .

\section{Procedure for the Ni-Catalyzed Reductive Arylalkylation of 1a with the NHP Ester 2 j on 1-mmol}

\section{Scale}

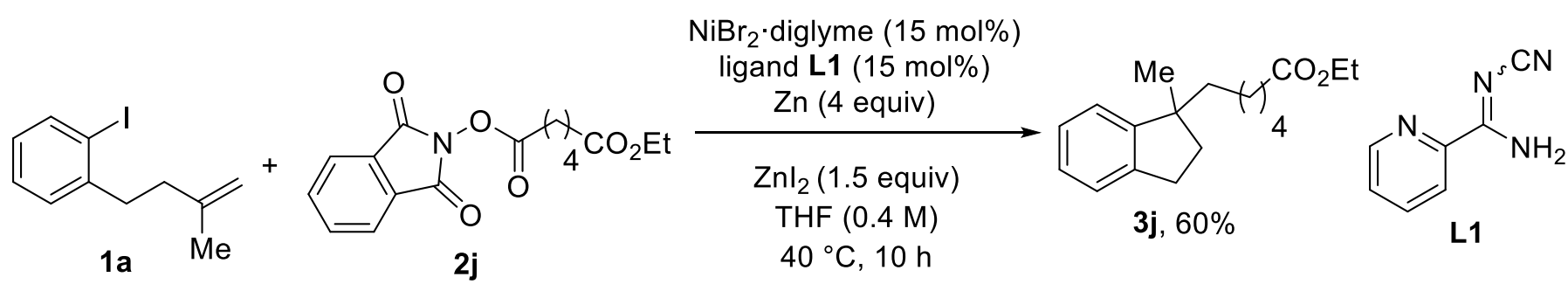

A sealed test tube charged with $\mathrm{NiBr}_{2} \bullet$ diglyme ( $\left.53 \mathrm{mg}, 0.15 \mathrm{mmol}, 15 \mathrm{~mol} \%\right)$, ligand $\mathbf{L 1}(22.5 \mathrm{mg}, 0.15 \mathrm{mmol}, 15 \mathrm{~mol} \%)$, $\mathrm{ZnI}_{2}(480 \mathrm{mg}, 1.5 \mathrm{mmol}, 1.5$ equiv), Zn-powder (260 mg, $1.5 \mathrm{mmol}, 4.0$ equiv) and a stir bar was evacuated and filled with nitrogen (three cycles). Next, alkene $1 \mathbf{a}(272 \mathrm{mg}, 1.0 \mathrm{mmol}, 1.0$ equiv), NHP Ester $\mathbf{2 j}$ ( $554 \mathrm{mg}, 2.0 \mathrm{mmol}$, 2.0 equiv) and THF $(2.5 \mathrm{~mL})$ were added and the resulting mixture was stirred at $40{ }^{\circ} \mathrm{C}$ for 10 hours. The mixture was then filtered through a pad of Celite and concentrated under reduced pressure. The residue was purified through column chromatography on silica gel with petroleum ether to the mixture eluent of petroleum ether and ethyl acetate (20:1), affording the product $\mathbf{3} \mathbf{j}$ as a colorless oil (164 mg, $60 \%$ ). 


\section{Unsuccessful Substrates:}<smiles>C=C(C)CCCOc1ccccc1I</smiles>

$1 \mathrm{u}$<smiles>C=C(C)CCCCOc1ccccc1I</smiles>
1v<smiles>C/C=C\CCc1ccccc1I</smiles>

$1 x$<smiles>COc1ccc(CC(=O)ON2C(=O)c3ccccc3C2=O)cc1</smiles><smiles>COc1ccc2c(c1)c(CC(=O)ON1C(=O)c3ccccc3C1=O)c(C)n2C(=O)c1ccc(Cl)cc1</smiles><smiles>CC1(C(=O)ON2C(=O)c3ccccc3C2=O)CCCCC1</smiles><smiles>CC(C)(Oc1ccc(Cl)cc1)C(=O)ON1C(=O)c2ccccc2C1=O</smiles> 


\section{Control Experiments for the Ni-catalyzed Reductive Arylalkylation Involving Decarboxylative}

\section{Coupling}

(a) Stoichiometric Reaction of Iodide 1a with Zn-Powder

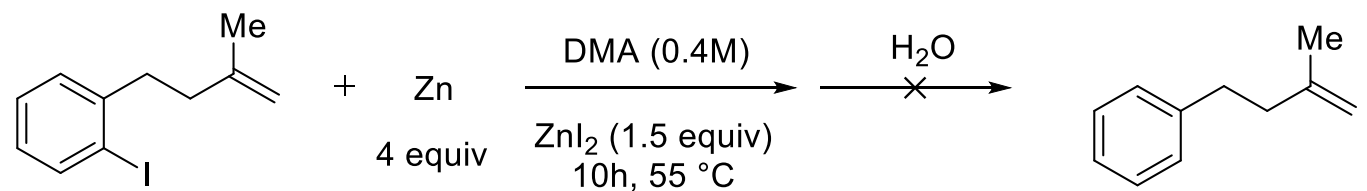

$1 \mathrm{a}$

To a sealed test tube containing $\mathrm{Zn}\left(52 \mathrm{mg}, 0.8 \mathrm{mmol}\right.$, 4.0 equiv) and $\mathrm{ZnI}_{2}$ (96 mg, 1.5 equiv) were added 1-iodo-2-(3methylbut-3-en-1-yl)benzene (1a) (54 mg, $0.2 \mathrm{mmol}, 1.0$ equiv) and THF ( $0.5 \mathrm{~mL})$, sequentially. The mixture was stirred at $40{ }^{\circ} \mathrm{C}$ for $10 \mathrm{~h}$ and then quenched with $\mathrm{H}_{2} \mathrm{O}$ and diluted with EtOAc $(5 \mathrm{~mL})$. The organic layers was then subjected to GC analysis.

(b) Stoichiometric Reactions of the Aryl Iodides 1a with Ni-Precatalysts Followed by Quenching with Water

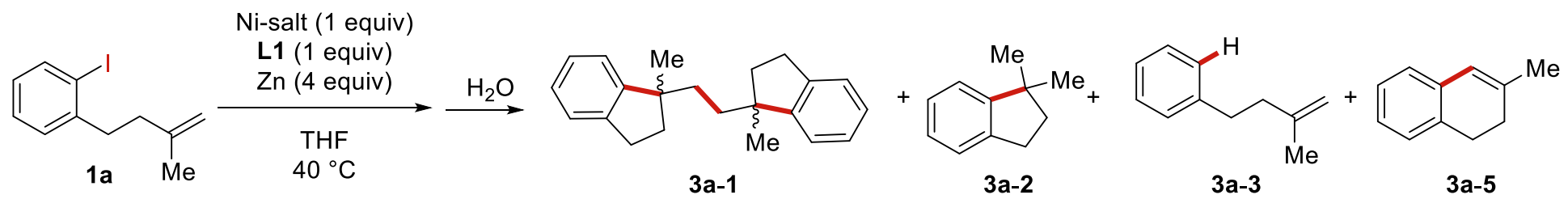

\begin{tabular}{|c|c|c|c|c|c|c|c|c|}
\hline Entry & Ni-precatalyst & additive & $\mathrm{t}(\mathrm{h})$ & yield $\mathbf{1 a}(\%)$ & yield 3a-1 (\%) & yield 3a-2 (\%) & yield $\mathbf{3 a} \mathbf{- 3}(\%)$ & yield 3a-5 (\%) \\
\hline $1^{\mathrm{a}}$ & $\mathrm{Ni}(\mathrm{COD})_{2}$ & - & 1 & 25 & 33 & 19 & 7 & 16 \\
\hline 2 & $\mathrm{Ni}(\mathrm{COD})_{2}$ & - & 1 & 21 & 49 & 13 & 9 & 8 \\
\hline 3 & $\mathrm{Ni}(\mathrm{COD})_{2}$ & $\mathrm{ZnI}_{2}$ & 1 & 99 & trace & trace & trace & 0 \\
\hline 4 & $\mathrm{Ni}(\mathrm{COD})_{2}$ & $\mathrm{ZnI}_{2}$ & 10 & 39 & 6 & 47 & 7 & 1 \\
\hline 5 & $\mathrm{NiBr}_{2} \bullet$ diglyme & - & 1 & 93 & 0 & 0 & 7 & 0 \\
\hline 6 & $\mathrm{NiBr}_{2} \bullet$ diglyme & - & 10 & 0 & 49 & 31 & 11 & 9 \\
\hline 7 & $\mathrm{NiBr}_{2} \bullet$ diglyme & $\mathrm{ZnI}_{2}$ & 3 & 0 & 16 & 67 & 11 & 6 \\
\hline
\end{tabular}

${ }^{a}$ Reaction was performed without $\mathrm{Zn}$.

To a sealed test tube containing Ni-salt ( $0.2 \mathrm{mmol}, 1.0$ equiv), $\mathbf{L 1}\left(29 \mathrm{mg}, 0.2 \mathrm{mmol}, 1.0\right.$ equiv), $\mathrm{ZnI}_{2}$ ( 0 or 1.5 equiv) and $\mathrm{Zn}$ (52 mg, $0.8 \mathrm{mmol}, 4$ equiv) were added 1-iodo-2-(3-methylbut-3-en-1-yl)benzene (1a) (54 mg, $0.2 \mathrm{mmol}, 1.0$ equiv) and THF $(0.5 \mathrm{~mL})$, sequentially. The mixture was stirred at $40{ }^{\circ} \mathrm{C}$ for $\mathrm{x} \mathrm{h}\left(\mathrm{x}=1,3\right.$ or 10) and then quenched with $\mathrm{H}_{2} \mathrm{O}$ and diluted with EtOAc $(5 \mathrm{~mL})$. The organic layers was then subjected to GC analysis. 


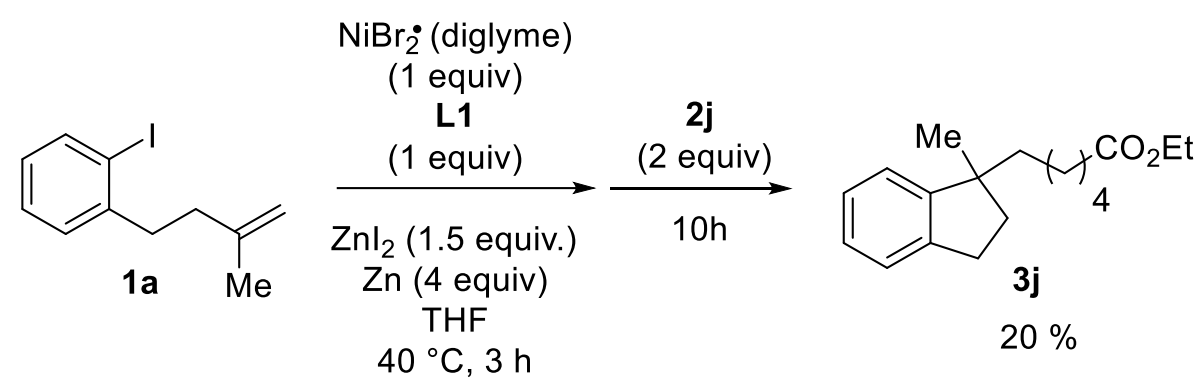

To a sealed test tube containing $\mathrm{NiBr}_{2}$. diglyme ( $75.4 \mathrm{mg}, 0.2 \mathrm{mmol}, 1.0$ equiv), $\mathbf{L 1}$ (29 mg, $0.2 \mathrm{mmol}, 1.0$ equiv), ZnI 2 (96 mg, 1.5 equiv) and Zn (52 mg, $0.8 \mathrm{mmol}, 4.0$ equiv) were added 1-iodo-2-(3-methylbut-3-en-1-yl)benzene (1a) (54 mg, 0.2 mmol, 1.0 equiv) and THF $(0.5 \mathrm{~mL})$, sequentially. The mixture was stirred at $40{ }^{\circ} \mathrm{C}$ for $3 \mathrm{~h}$ before 1,3-dioxoisoindolin-2-yl ethyl adipate (2j) $\left(127.6 \mathrm{mg}, 0.4 \mathrm{mmol}, 2.0\right.$ equiv) was added. The mixture was stirred at $40{ }^{\circ} \mathrm{C}$ for $10 \mathrm{~h}$ and then quenched with $\mathrm{H}_{2} \mathrm{O}$ and diluted with EtOAc $(5 \mathrm{~mL})$. The organic layers was then subjected to $\mathrm{GC}$ analysis using $n$-dodecane as an internal standard.

\section{(d) Reaction between NHP Ester 2h and (3-Methylbut-3-en-1-yl)benzene}

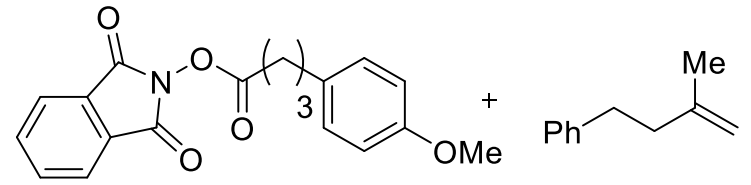

$2 \mathrm{~h}$

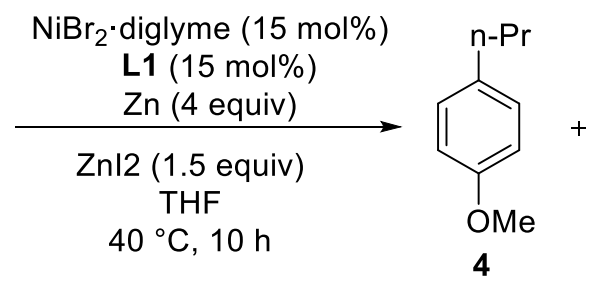

$31 \%$<smiles>COc1ccc(CC(C)C(C)C(C)(C)c2ccccc2)cc1</smiles>

$0 \%$

To a sealed test tube charged with $\mathrm{NiBr}_{2} \cdot \operatorname{diglyme}(10.6 \mathrm{mg}, 0.06 \mathrm{mmol}, 15 \mathrm{~mol} \%)$, ligand $\mathbf{L 1}$ (4.5 mg, $\left.0.06 \mathrm{mmol}, 15 \mathrm{~mol} \%\right)$, $\mathrm{ZnI}_{2}$ (96 mg, 1.5 equiv), Zn-powder (52mg, $0.8 \mathrm{mmol}, 4.0$ equiv), 1,3-dioxoisoindolin-2-yl 4-(4-methoxyphenyl)butanoate (135.6 mg, $0.4 \mathrm{mmol}, 2.0$ equiv) and (3-methylbut-3-en-1-yl)benzene ( $29.2 \mathrm{mg}, 0.2 \mathrm{mmol}, 1.0$ equiv) was added THF (0.5 $\mathrm{ml}$ ), and the resulting mixture was stirred at $40{ }^{\circ} \mathrm{C}$ for $10 \mathrm{~h}$, before it was quenched with $\mathrm{H}_{2} \mathrm{O}$ and diluted with EtOAc $(5$ $\mathrm{mL})$. The organic layer was then subjected to $\mathrm{GC}$ analysis using $n$-dodecane as an internal standard. ${ }^{1} \mathrm{H} \mathrm{NMR}(400 \mathrm{MHz}$, Chloroform-d) $\delta=7.18-7.08(\mathrm{~m}, 2 \mathrm{H}), 6.92-6.81(\mathrm{~m}, 2 \mathrm{H}), 3.82(\mathrm{~s}, 3 \mathrm{H}), 2.56(\mathrm{t}, J=7.6 \mathrm{~Hz}, 2 \mathrm{H}), 1.73-1.55(\mathrm{~m}, 2 \mathrm{H}), 0.96(\mathrm{t}, J$ $=7.3 \mathrm{~Hz}, 3 \mathrm{H}) \mathrm{ppm} .{ }^{13} \mathrm{C} \mathrm{NMR}\left(101 \mathrm{MHz}, \mathrm{CDCl}_{3}\right) \delta=157.6,134.8,129.3$ (2C), $113.6(2 \mathrm{C}), 55.2,37.2,24.8,13.8 \mathrm{ppm}$. 


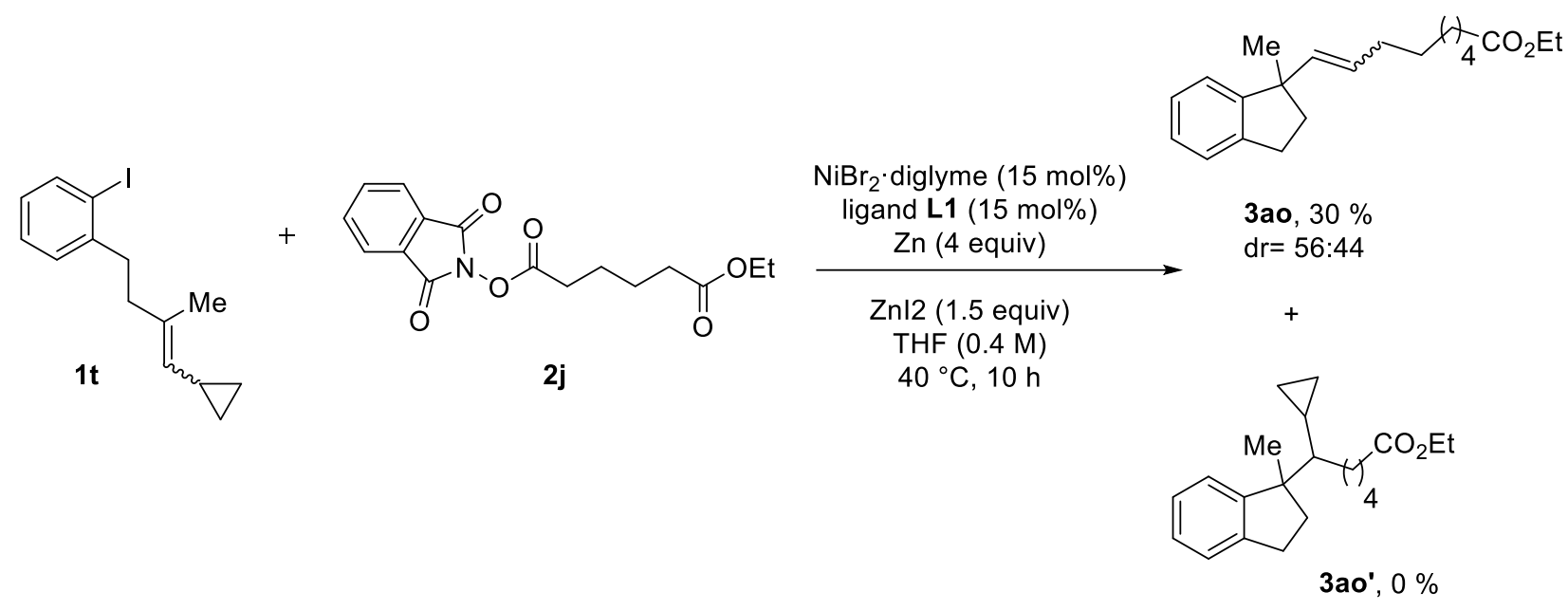

To a sealed test tube containing $\mathrm{NiBr}_{2} \cdot$ diglyme (10.6 mg, $\left.0.06 \mathrm{mmol}, 15 \mathrm{~mol} \%\right), \mathbf{L 1}$ (4.5 mg, $\left.0.06 \mathrm{mmol}, 15 \mathrm{~mol} \%\right), \mathrm{ZnI}_{2}(96$ $\mathrm{mg}, 1.5$ equiv) and $\mathrm{Zn}(52 \mathrm{mg}, 0.8 \mathrm{mmol}, 4.0$ equiv) were added 1-(4-cyclopropyl-3-methylbut-3-en-1-yl)-2-iodobenzene (1t) (62 mg, $0.2 \mathrm{mmol}, 1.0$ equiv), 1,3-dioxoisoindolin-2-yl ethyl adipate (2j) (127.6 mg, $0.4 \mathrm{mmol}, 2.0$ equiv) and THF ( $0.5 \mathrm{~mL}$ ), sequentially. The mixture was stirred at $40{ }^{\circ} \mathrm{C}$ for $10 \mathrm{~h}$ and then quenched with $\mathrm{H}_{2} \mathrm{O}$. The aqueous phase was then extracted with EtOAc ( $3 \times 10 \mathrm{~mL})$. The combined organic layers were washed with $\mathrm{H}_{2} \mathrm{O}(3 \times 5 \mathrm{~mL})$, dried over $\mathrm{Na}_{2} \mathrm{SO}_{4}$, filtered and concentrated in vacuum. The residue was purified by silica gel column chromatography with eluent of petroleum ether to the mixture of petroleum ether and ethyl acetate (20:1) to afford the product $3 \mathbf{a o}$ (19 $\mathrm{mg}, 30 \%$ yield) as a yellow oil.

${ }^{1} \mathrm{H}$ NMR: (400 MHz, Chloroform- $d$ ) $\delta=7.24-7.10(\mathrm{~m}, 3 \mathrm{H}), 7.11-7.02(\mathrm{~m}, 1 \mathrm{H}), 5.62-5.49(\mathrm{~m}, 1 \mathrm{H}), 5.34-5.03(\mathrm{~m}, 1 \mathrm{H}), 4.12(\mathrm{q}$, $J=7.1 \mathrm{~Hz}, 2 \mathrm{H}), 2.93-2.80(\mathrm{~m}, 2 \mathrm{H}), 2.32-2.23(\mathrm{~m}, 2 \mathrm{H}), 2.13-1.86(\mathrm{~m}, 3 \mathrm{H}), 1.67-1.51(\mathrm{~m}, 2 \mathrm{H}), 1.37-1.18(\mathrm{~m}, 6 \mathrm{H}), 1.24(\mathrm{t}, J=$ $7.1 \mathrm{~Hz}, 3 \mathrm{H}), 1.22(\mathrm{~s}, 3 \mathrm{H}), 0.94(\mathrm{~d}, J=6.7 \mathrm{~Hz}, 1 \mathrm{H}) \mathrm{ppm} .{ }^{13} \mathrm{C}$ NMR $(101 \mathrm{MHz}$, Chloroform- $d$ ): $\delta$ (major isomer) $=173.9,150.5$, 143.2, 138.1, 132.9, 127.0, 126.2, 124.5, 123.3, 60.2, 49.5, 40.9, 36.7, 34.4, 32.5, 30.1, 29.00, 25.9, 25.00, 20.9, 14.3 ppm; $\delta$ (minor isomer $)=173.9,150.5,143.2,138.1,132.9,127.0,126.3,124.5,123.3,60.2,49.4,41.00,36.6,34.4,32.5,29.4,28.7,26.9,25.1$, 21.1, 14.3 ppm. HRMS (ESI): calcd. for $\mathrm{C}_{21} \mathrm{H}_{31} \mathrm{O}_{2}{ }^{+}[\mathrm{M}+\mathrm{H}]^{+}$: 315.2319, found: 315.2316. 


\section{Proposed Reaction Mechanism}

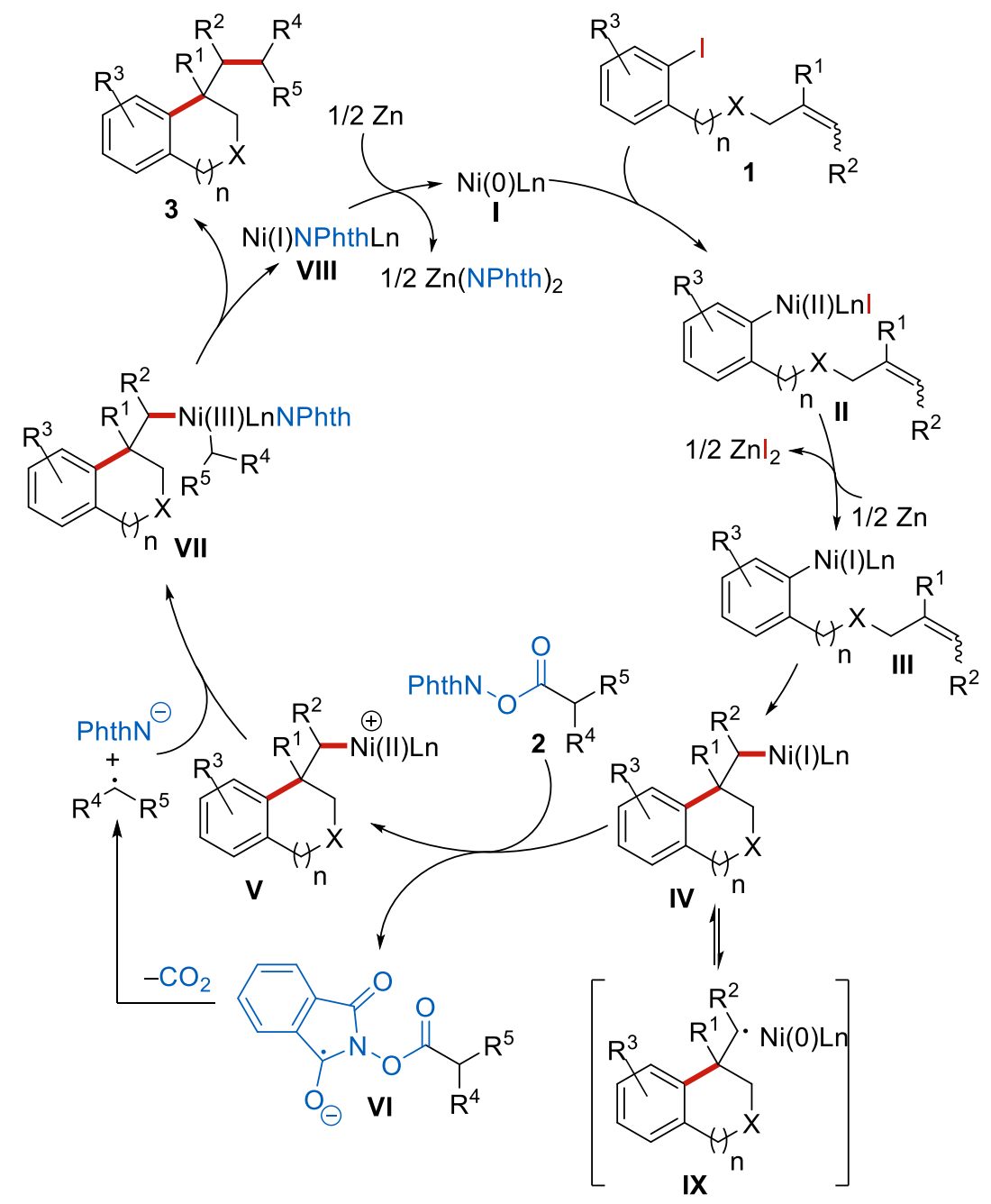

On the basis of control experiments, we proposed the following plausible mechanism for this Ni-catalyzed reaction (Scheme 5). Initially, a $\mathrm{Ni}(0)$ species I is generated under reductive reaction conditions, which undergoes the oxidative addition with aryl iodides $\mathbf{1}$. In the next step, the $\mathrm{Zn}$-mediated reduction of the generated $\mathrm{Ni}$ (II) complex II gives rise to the formation of a $\mathrm{Ni}(\mathrm{I})$ intermediate III. ${ }^{16,17}$ Subsequently, the 5-exo cyclization is accomplished through a $\mathrm{Ni}(\mathrm{I})$-mediated migratory insertion. Next, the Ni(I)-alkyl species IV reduces the redox active NHP esters $\mathbf{2}$ through a sing electron transfer to afford a cationic $\mathrm{Ni}(\mathrm{II})$ complex $\mathbf{V}$ and a radical anion VII. ${ }^{18}$ Fragmentation of $\mathbf{V I}$ with the extrusion of $\mathrm{CO}_{2}$ provides a phthalimide and an alkyl radical for combination with the complex $\mathbf{V}$. Reductive elimination from the resultant $\mathrm{Ni}$ (III) intermediate VII provides the product 3 and a $\mathrm{Ni}(\mathrm{I})$ species VIII. Finally, the $\mathrm{Ni}(0)$ species I was regenerated through $\mathrm{Zn}$-mediated reduction. In addition, we assume that the $\mathrm{Ni}(\mathrm{I})$ complex IV stays in equilibrium with a cage IX, which leads to the high diastereoselectivities obtained for the products starting from trisubstituted olefins with nearly 1:1 E/Z ratios. The result of a radical clock reaction also supports this hypothesis. 


\section{Asymmetric Ni-catalyzed Reductive Arylalkylation involving Decarboxylative Coupling}

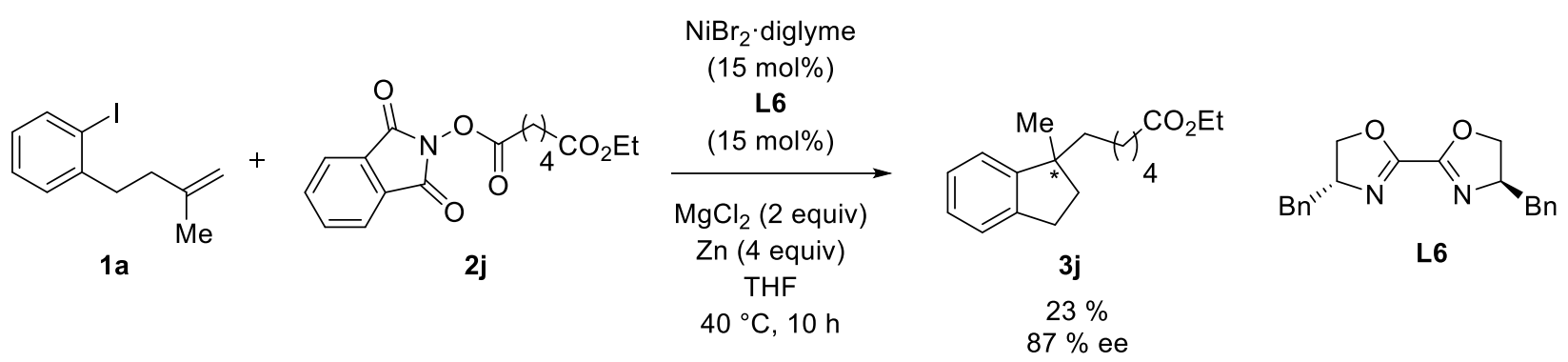

A sealed test tube charged with $\mathrm{NiBr}_{2} \cdot$ diglyme $(10.6 \mathrm{mg}, 0.03 \mathrm{mmol}, 15 \mathrm{~mol} \%)$, ligand $\mathbf{L 6}$ (9.6 $\left.\mathrm{mg}, 0.03 \mathrm{mmol}, 15 \mathrm{~mol} \%\right)$, $\mathrm{MgCl}_{2}$ (28.5 mg, $0.3 \mathrm{mmol}, 1.5$ equiv), $\mathrm{Zn}$-powder ( $52 \mathrm{mg}, 0.8 \mathrm{mmol}, 4.0$ equiv) and a stir bar was evacuated and filled with nitrogen (three cycles) before adding THF $(0.5 \mathrm{~mL})$. Next, 1,3-dioxoisoindolin-2-yl ethyl adipate (2j) $(127.6 \mathrm{mg}, 0.4 \mathrm{mmol}$, 2.0 equiv) and alkenes $1 \mathrm{a}\left(54.4 \mathrm{mg}, 0.2 \mathrm{mmol}, 1.0\right.$ equiv) were added and the resulting mixture was stirred at $40{ }^{\circ} \mathrm{C}$ for $10 \mathrm{~h}$. The mixture was then filtered through a pad of Celite and concentrated under reduced pressure. The residue was purified by column chromatography (petroleum ether/ethyl acetate $=20: 1)$ on silica gel to afford $\mathbf{3 j}$ as a colorless oil $(12.6 \mathrm{mg}, 23 \%$, $87 \% e e$ ). The $e e$ was determined by HPLC analysis (Chiralcel OJ-H column, $\lambda=254 \mathrm{~nm}$, hexane/isopropanol $=98 / 2$, flow rate $=0.5 \mathrm{~mL} / \mathrm{min}): \mathrm{t}_{\mathrm{R}}($ major $)=10.8 \mathrm{~min}, \mathrm{t}_{\mathrm{R}}($ minor $)=11.3 \mathrm{~min}$.

\section{References}

1. Tian, Z.-X.; Qiao, J.-B.; Xu, G.-L.; Pang, X.; Qi, L.; Ma, W.-Y.; Zhao, Z.-Z.; Duan, J.; Du, Y.-F.; Su, P.; Liu, X.-Y.; Shu, X.-Z. Highly enantioselective cross-Electrophile aryl-Alkenylation of unactivated Alkenes. J. Am. Chem. Soc. 2019, 141, 7637-7643.

2. Hu, M.; Gao, Y.; Wu, W.; Li, J.; Li, C.; Zhang, H.; Jiang, H. Efficient assembly of Ynones via Palladium-Catalyzed Sequential Carbonylation/Alkynylation. Org. Biomol. Chem. 2018, 16, 7383-7392.

3. Sickert, M.; Weinstab, H.; Peters, B.; Hou, X.; Lautens, M. Intermolecular Domino Reaction of Two Aryl Iodides Involving Two C-H Functionalizations. Angew. Chem. Int. Ed. 2014, 53, 5147-5151.

4. Ramesh, K.; Basuli, S.; Satyanarayana, G. Microwave-Assisted Domino Palladium Catalysis in Water: A Diverse Synthesis of 3,3'-Disubstituted Heterocyclic Compounds. Eur. J. Org. Chem. 2018, 2171-2177.

5. Negishi, E.-i.; Copéret, C.; Ma, S.; Mita, T.; Sugihara, T.; Tour, J. M. Palladium-Catalyzed Carbonylative Cyclization of 1-Iodo-2-alkenylbenzenes. J. Am. Chem. Soc. 1996, 118, 5904-5918.

6. Zheng, H.-X.; Shan, X.-H.; Qu, J.-P.; Kang, Y.-B. Transition-Metal-Free Hydrogenation of Aryl Halides: From Alcohol to Aldehyde. Org. Lett. 2017, 19, 5114-5117.

7. (a) Lu, X..; Xiao, B.; Liu, L.; Fu, Y. Formation of $\mathrm{C}\left(\mathrm{sp}^{3}\right)-\mathrm{C}\left(\mathrm{sp}^{3}\right)$ Bonds through Nickel-Catalyzed Decarboxylative Olefin Hydroalkylation Reactions Chem. Eur. J. 2016, 22, 11161-11164. (b) Lu, X.; Wang, X.-Xu; Gong, T.-J.; Pi, J.-J.; He, S.-J.; Fu, Y. Nickel-Catalyzed Allylic Defluorinative Alkylation of Trifluoromethyl Alkenes with Reductive Decarboxylation of Redox-Active Esters. Chem. Sci., 2019, 10, 809-814.

8. Hansen, E. C.; Pedro, D. J.; Wotal, A. C.; Gower, N. J.; Nelson, J. D.; Caron, S.; Weix, D. J. New Ligands for Nickel Catalysis from Diverse Pharmaceutical Heterocycle Libraries. Nature Chem. 2016, 8, 1126-1130.

9. Whyte, A.; Olson, M. E.; Lautens, M. Palladium-Catalyzed, Norbornene-Mediated, ortho-Amination ipso-Amidation: Sequential C-N Bond Formation. Org. Lett. 2018, 20, 345-348.

10. Yong, K. H.; Lotoski, J. A.; Chong, J. M. Studies on the Alkylation of 3-Methyl-3-buten-1-ol Dianion: an Efficient Synthesis of 3-Methylene-1-alkanols Including a San Jose Scale Sex Pheromone. J. Org. Chem. 2001, 66, 8248-8251.

11. Bergström, M.; Suresh, G.; Naidu, V. R.; Unelius, C. R. N-iodosuccinimide (NIS) in Direct Aromatic Iodination. Eur. J. Org. Chem. 2017, 3234-3239.

12. Konishi, H.; Tanaka, H.; Manabe, K. Pd-catalyzed Selective Synthesis of Cyclic Sulfonamides and Sulfinamides using 
$\mathrm{K}_{2} \mathrm{~S}_{2} \mathrm{O}_{5}$ as a Sulfur Dioxide Surrogate. Org. Lett. 2017, 19, 1578-1581.

13. Márquez, I. R.; Miguel, D.; Millán, A.; Marcos, M. L.; de Cienfuegos, L. Á.; Campaña, A. G.; Cuerva, J. M. Ti/Nimediated Inter- and intramolecular Conjugate Addition of Aryl and Alkenyl Halides and Triflates. J. Org. Chem. 2014, 79, 1529-1541.

14. Ghosh, A. K.; Nicponski, D. R., Cu(II)-catalyzed Olefin Migration and Prins Cyclization: Highly Diastereoselective Synthesis of Substituted Tetrahydropyrans. Org. Lett. 2011, 13, 4328-4331.

15. (a) Jin, Y.; Wang, C. Ni-catalysed Reductive Arylalkylation of Unactivated Alkenes. Chem. Sci. 2019, 10, 1780-1785. (b) Jin. Y.; Wang, C. Nickel-catalyzed Asymmetric Reductive Arylalkylation of Unactivated Alkenes. Angew. Chem. Int. Ed. 2019, $58,6722-6726$.

16. Colon, I.; Kelsey, D. R. Mechanism of Oxidative Coupling of Aryl Chlorides by Nickel and Reducing Metals. J. Org. Chem. 1986, 51, 2627-2637.

17. Liu, J.; Ren, Q.; Zhang, X.; Gong, H. Preparation of Vinyl Arenes by Nickel-Catalyzed Reductive Coupling of Aryl Halides with Vinyl Bromides. Angew. Chem. Int. Ed. 2016, 55, 15544-15548.

18. Cornella, J.; Edwards, J. T.; Qin, T.; Kawamura, S.; Wang, J.; Pan, C.-M.; Gianatassio, R.; Schmidt, M.; Eastgate, M. D.; Baran, P. S. Practical Ni-Catalyzed Aryl-Alkyl Cross-Coupling of Secondary Redox-Active Esters. J. Am. Chem. Soc. 2016, $138,2174-2177$. 
${ }^{1} \mathrm{H}$ and ${ }^{13} \mathrm{C}$ NMR Spectra

×

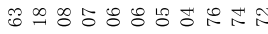

rinin

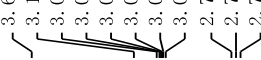

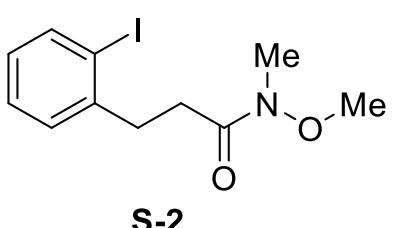

S-2

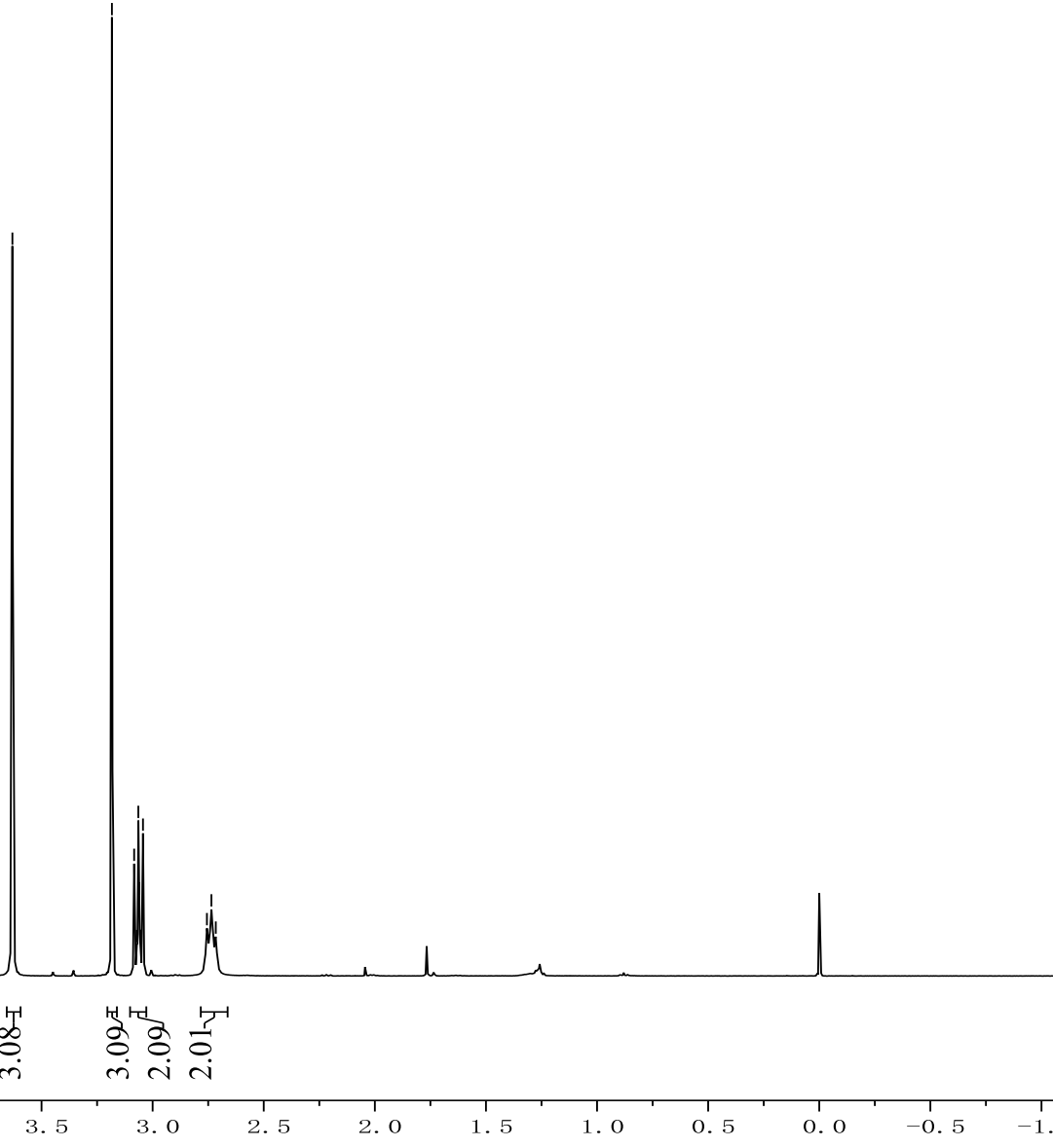



$\infty \infty$

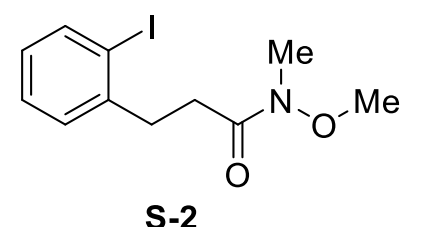

S-2
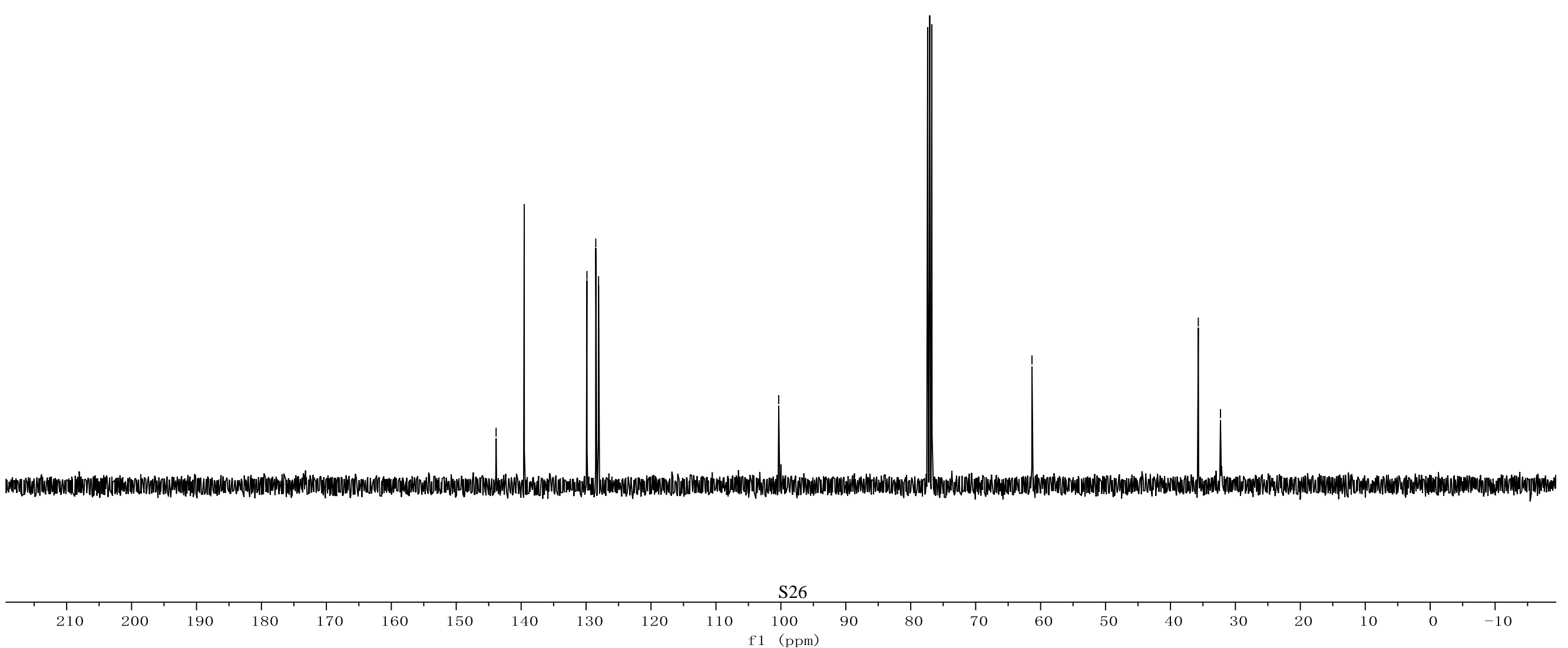


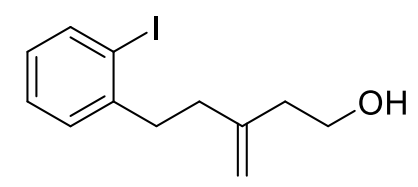

S-4

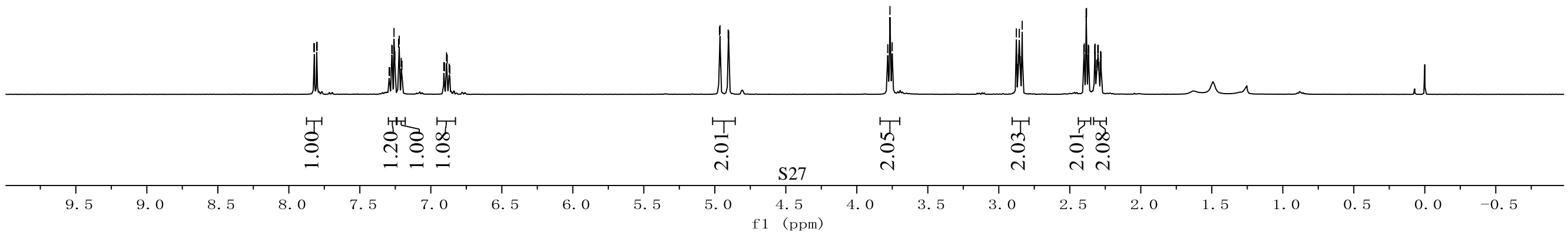




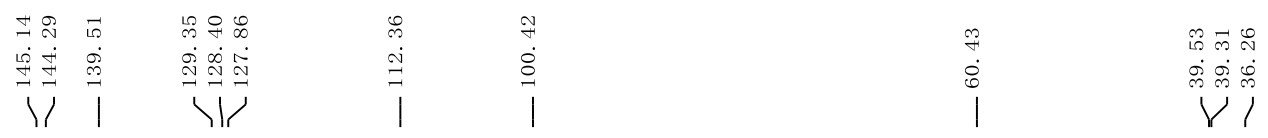

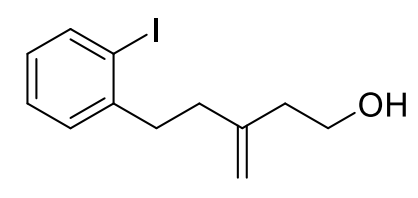

S-4
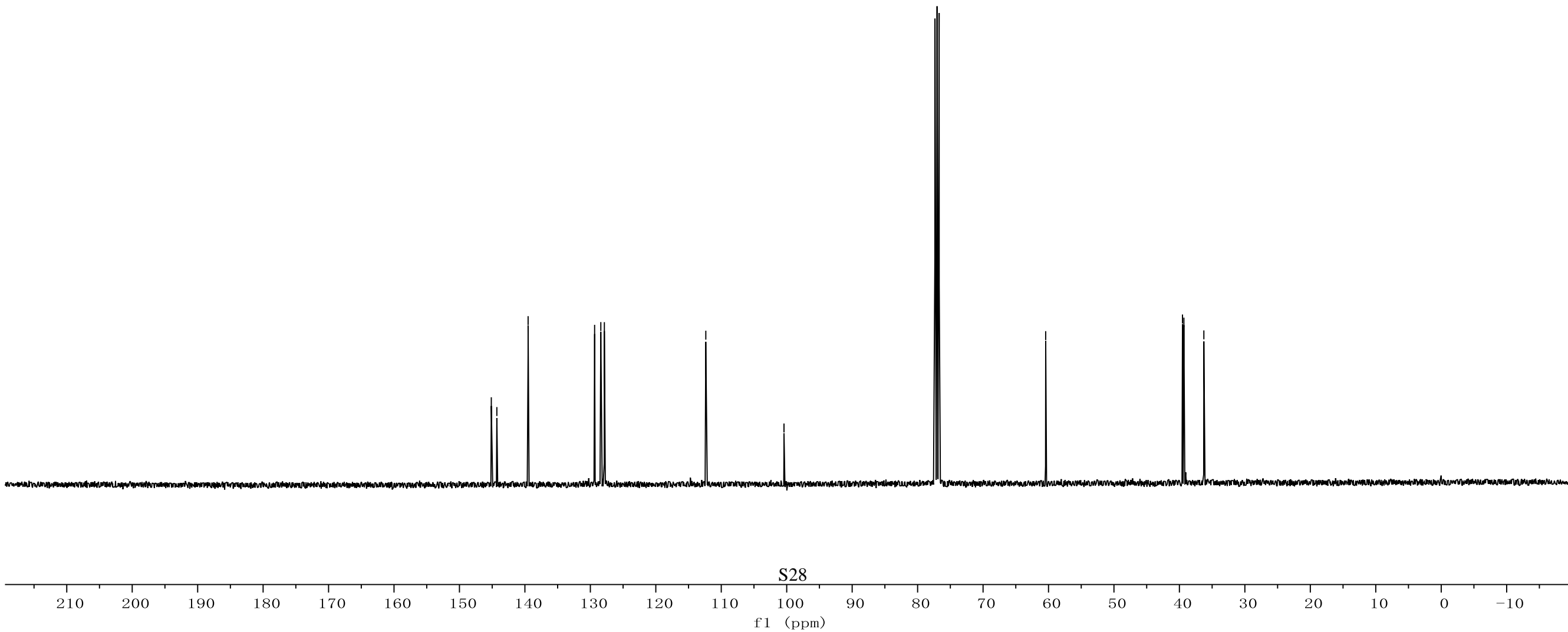


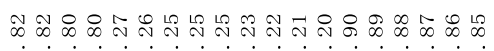

$\underbrace{-10}$
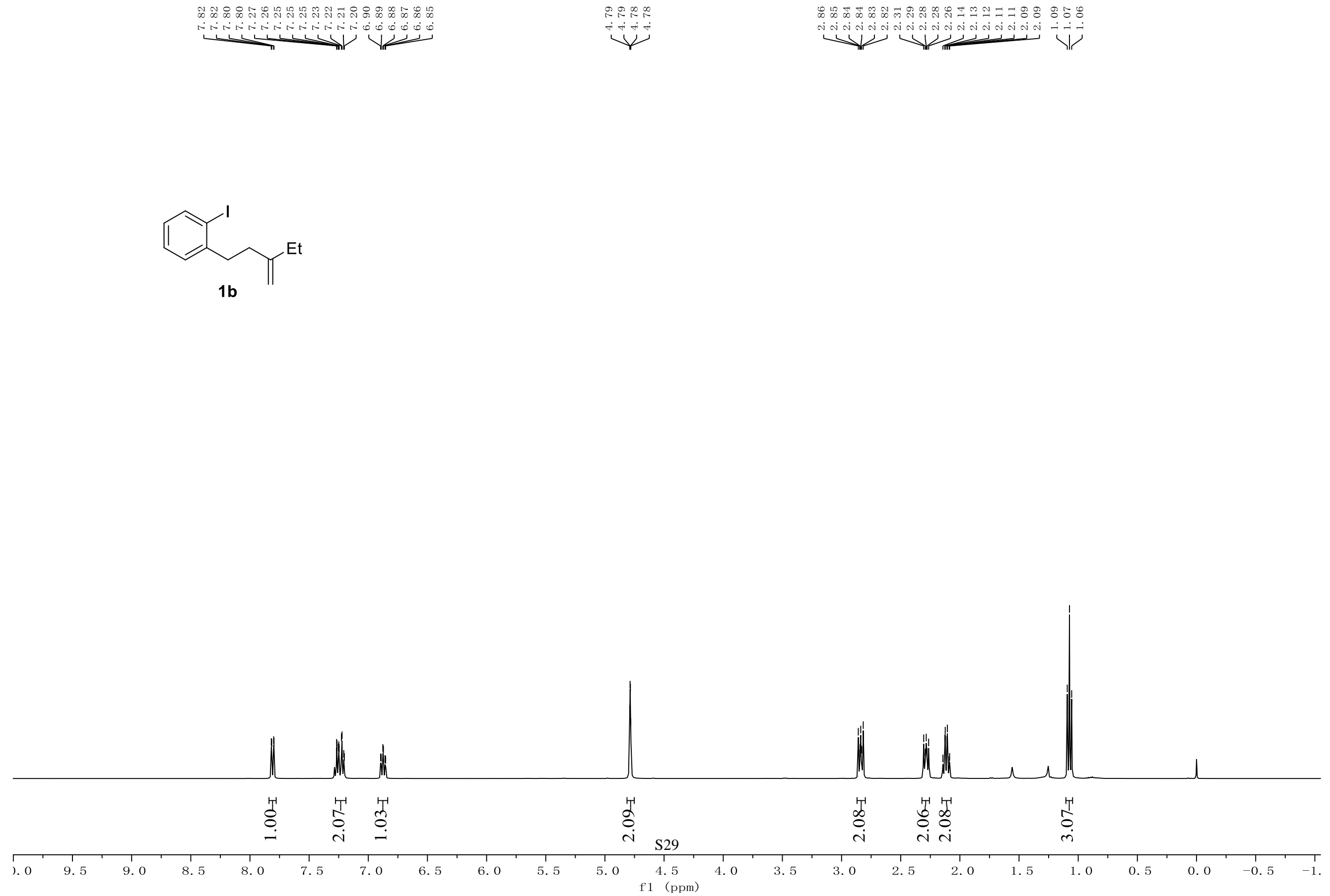


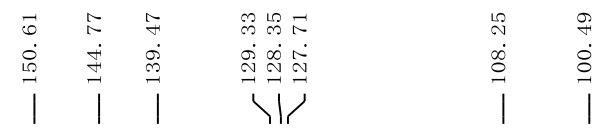

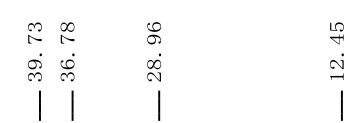

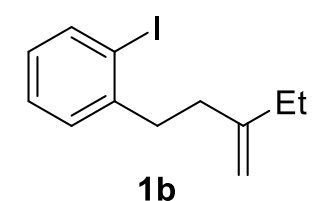

1b
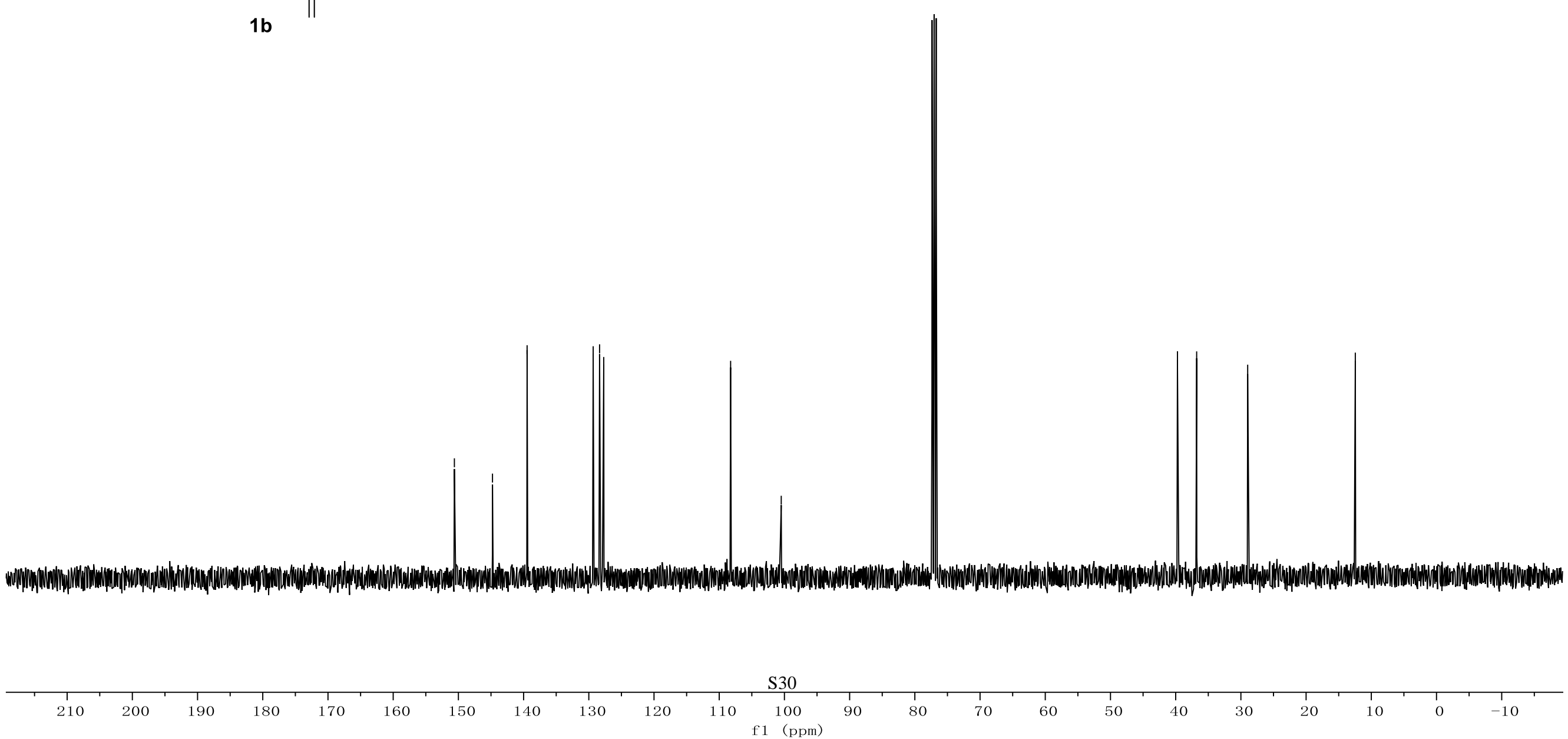

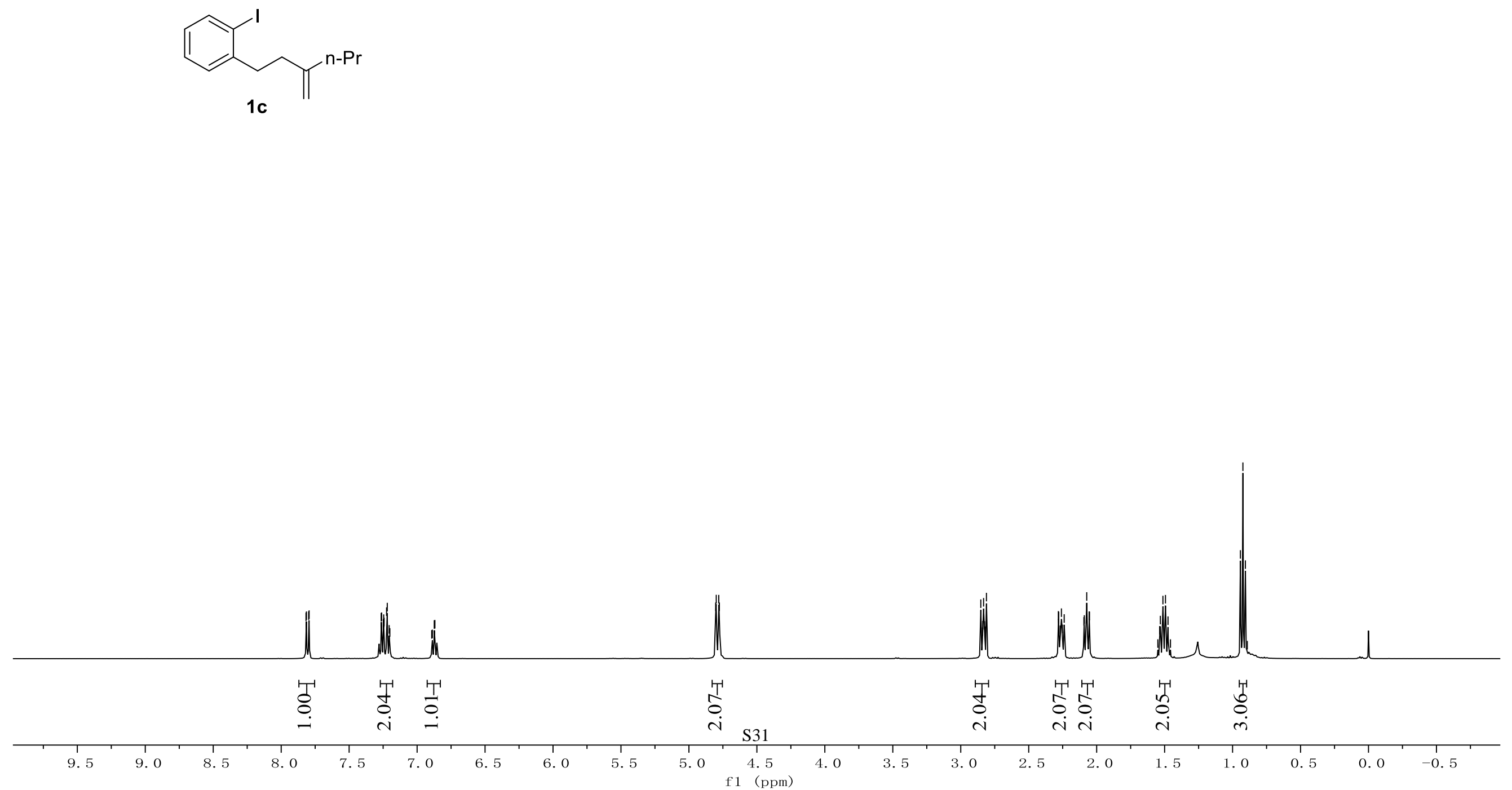


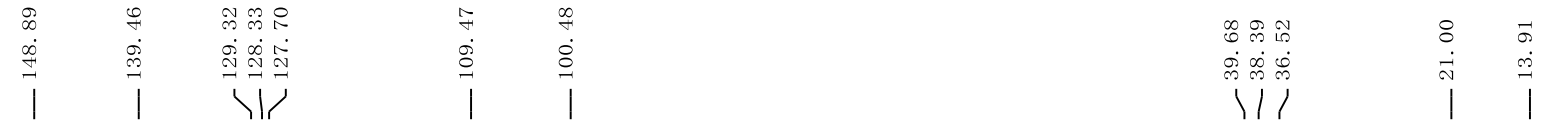

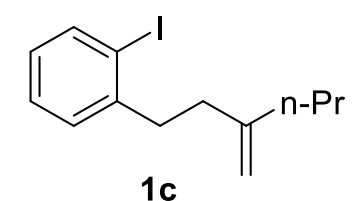

1c

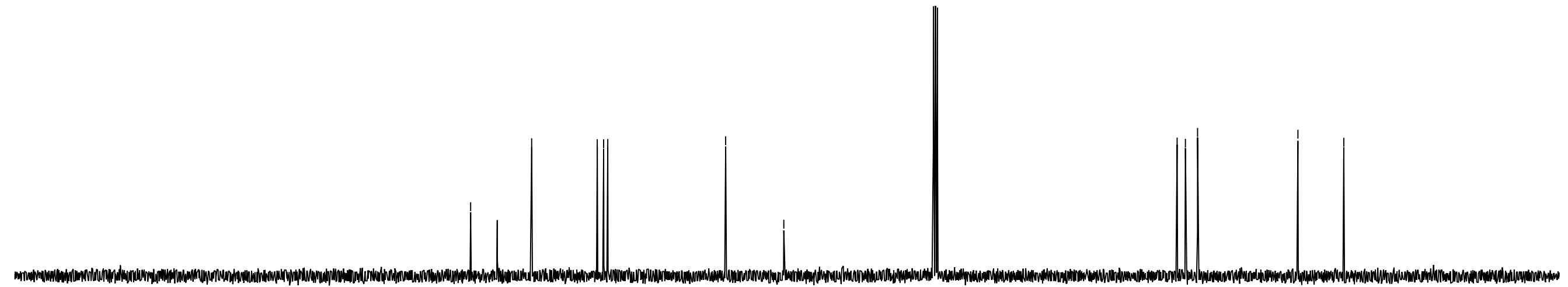

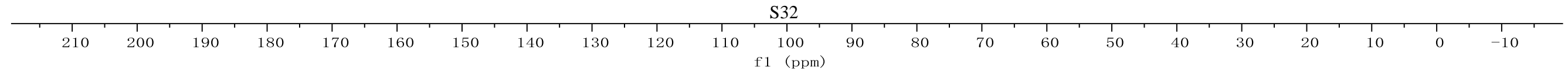



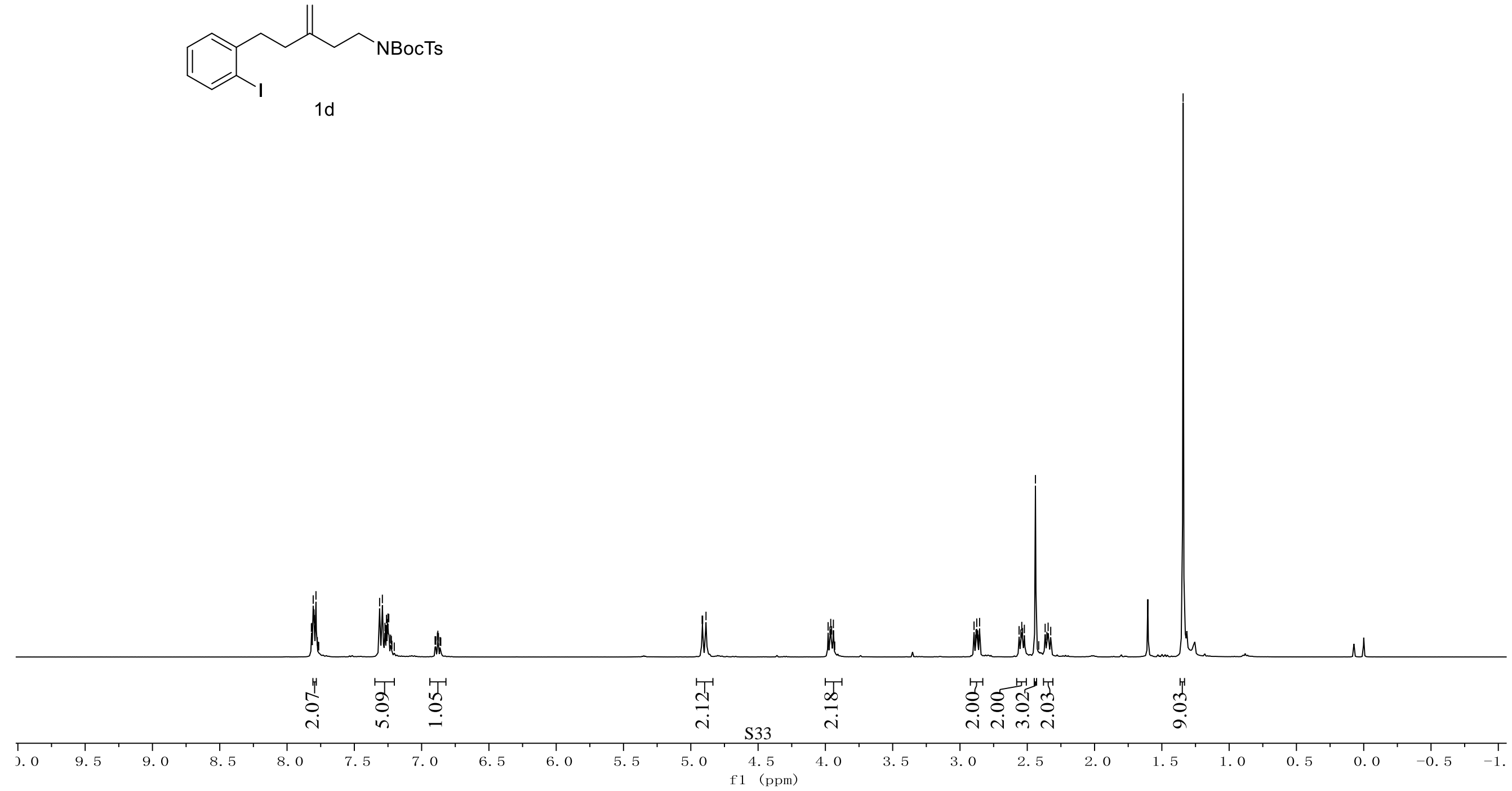


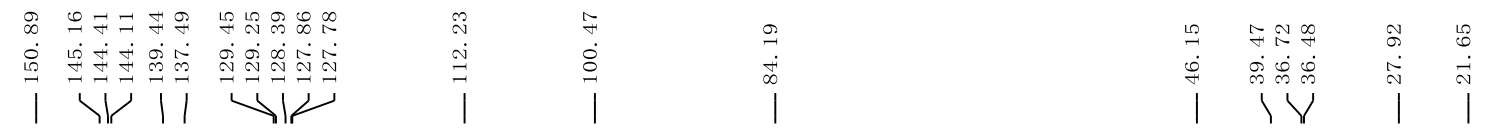
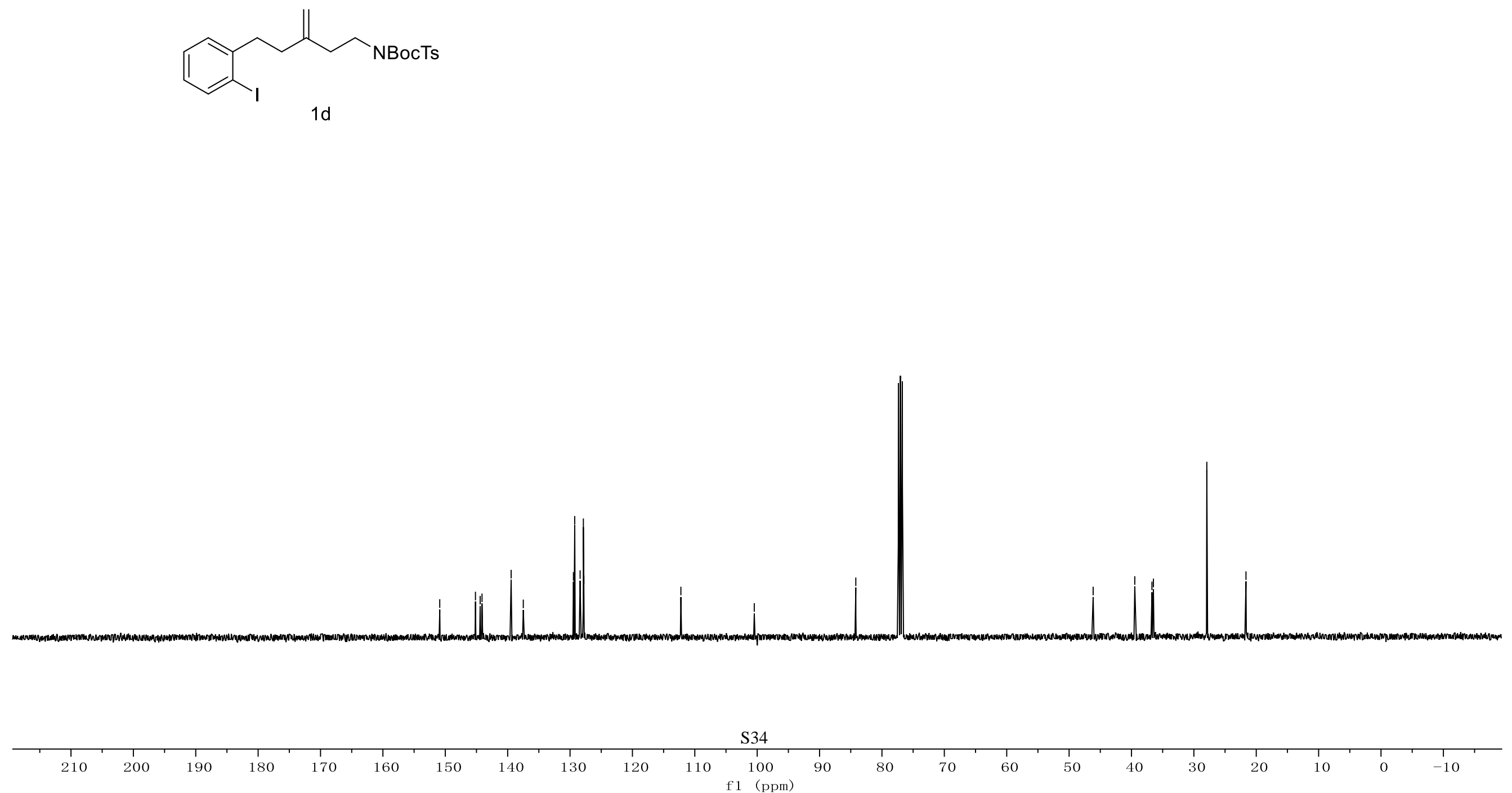

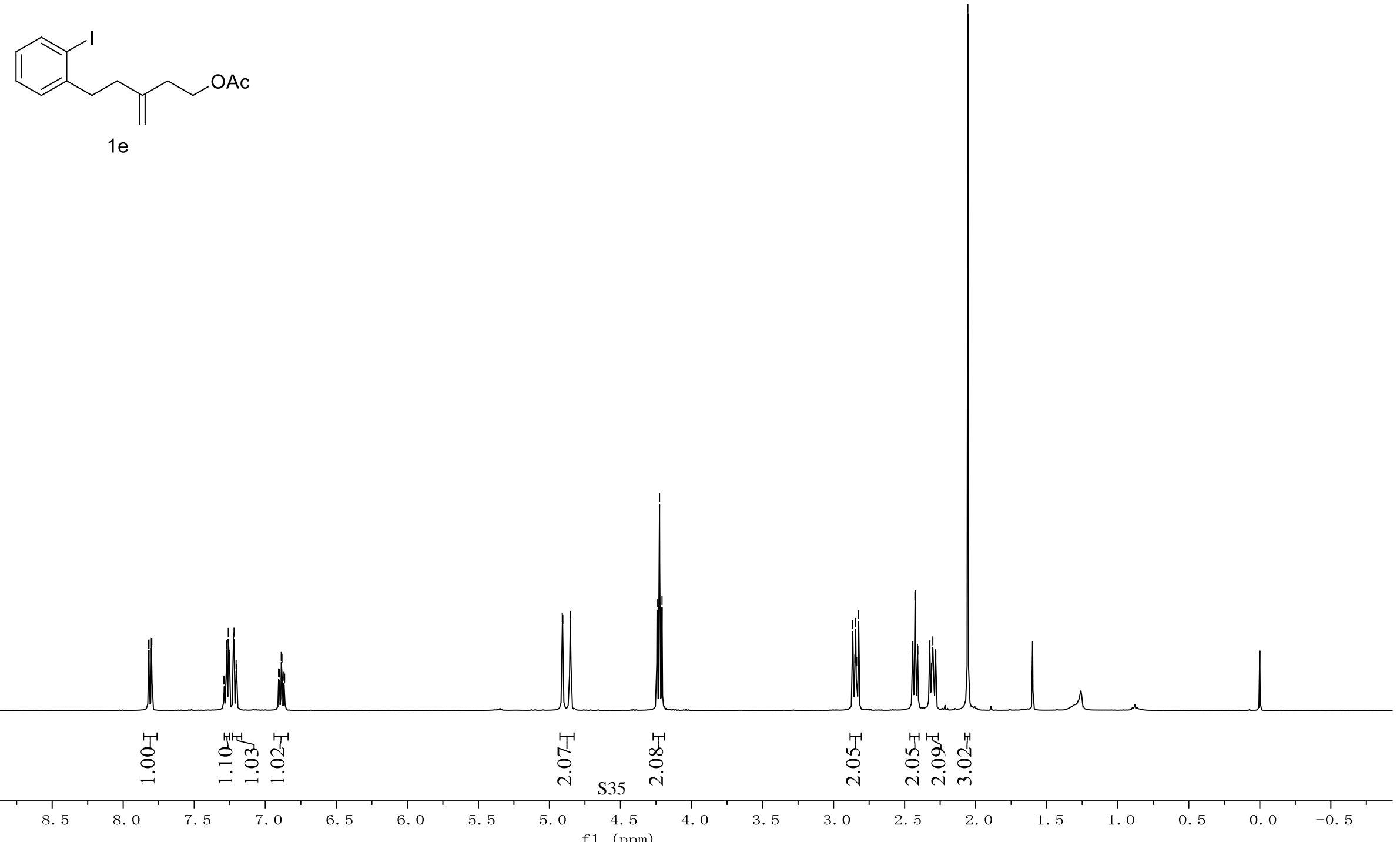


$$
\text { يل. }
$$



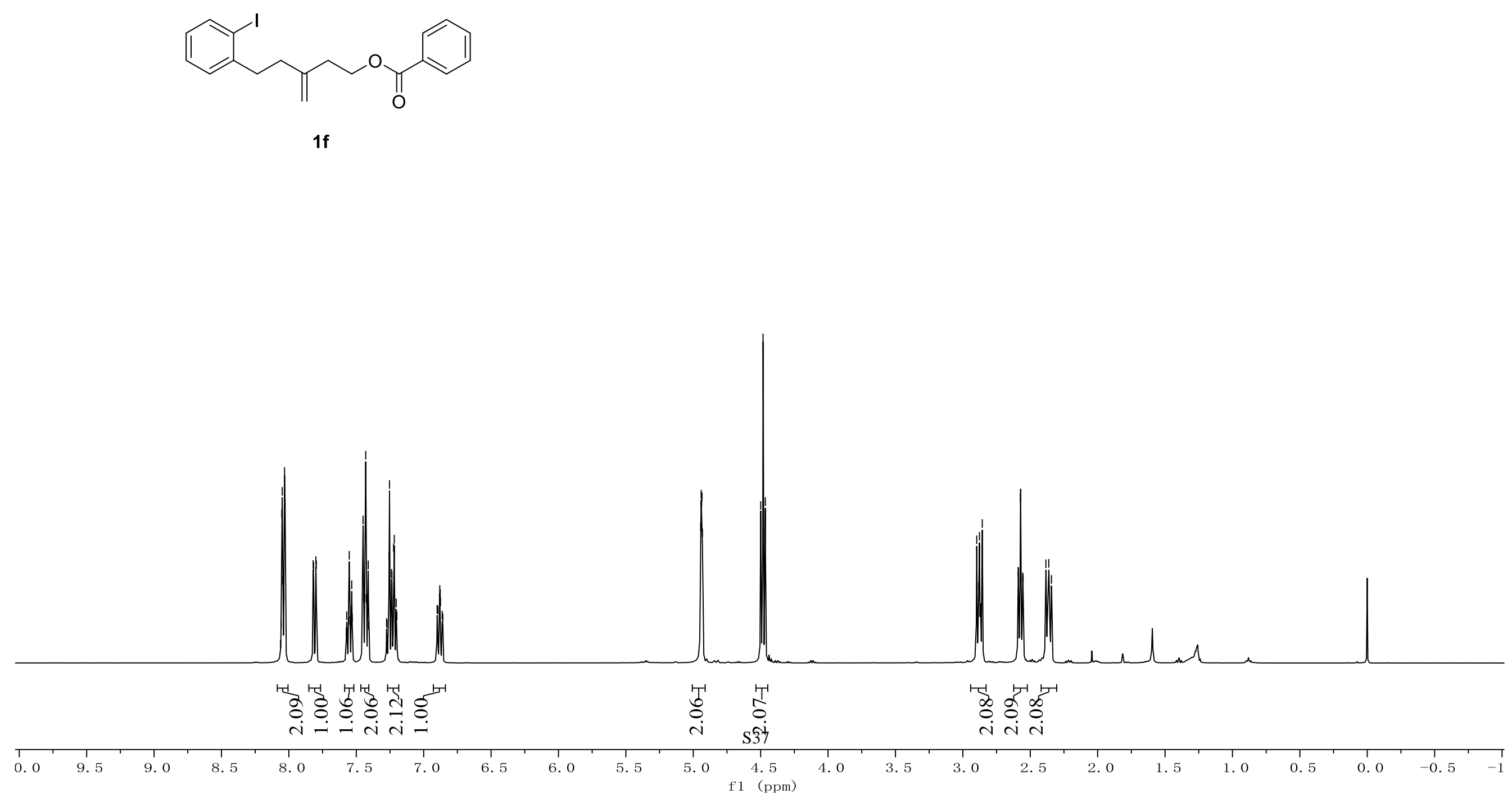


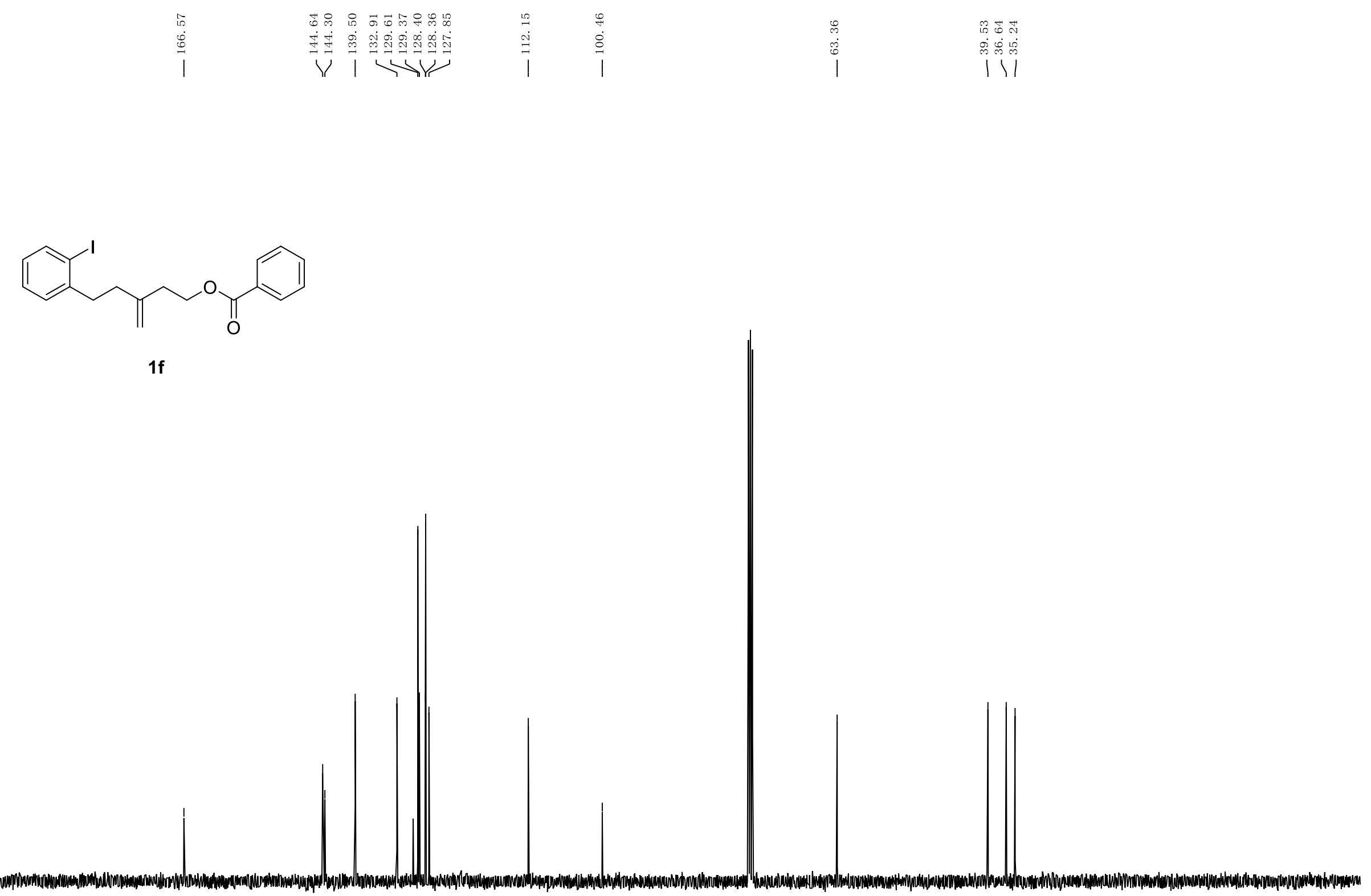

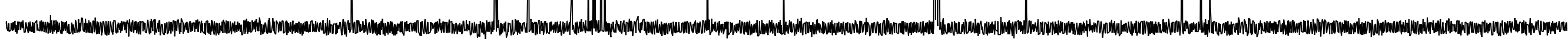

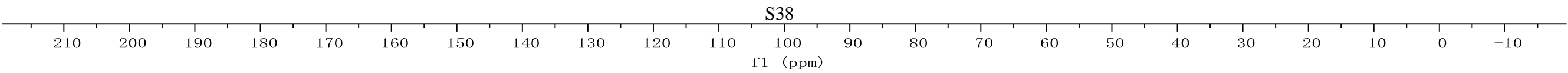




$$
\text { arpopes }
$$

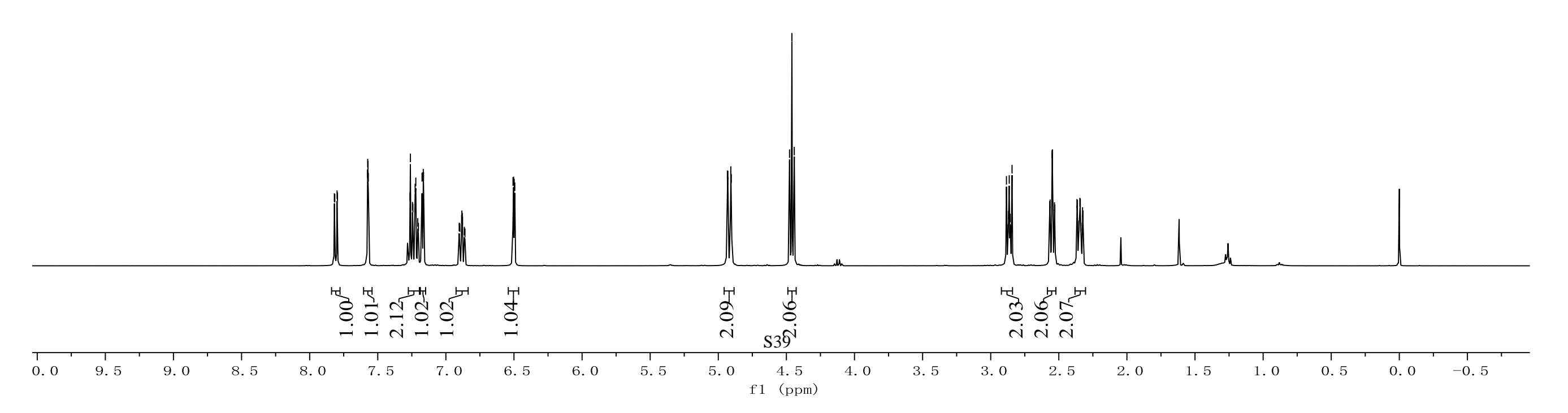




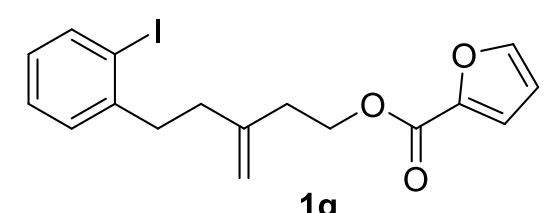

19

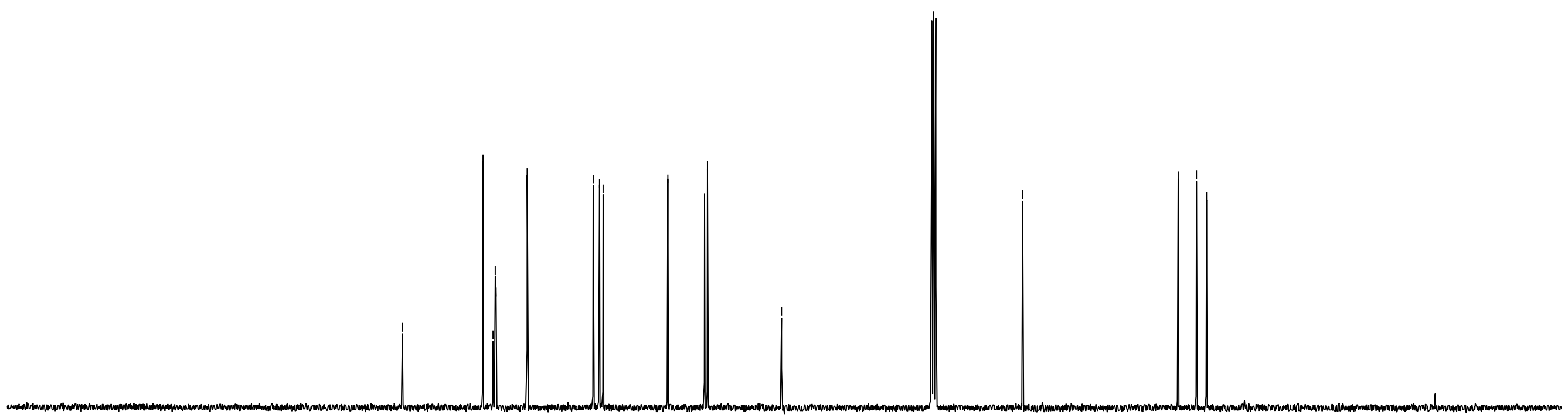

S40

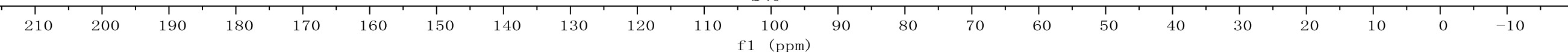




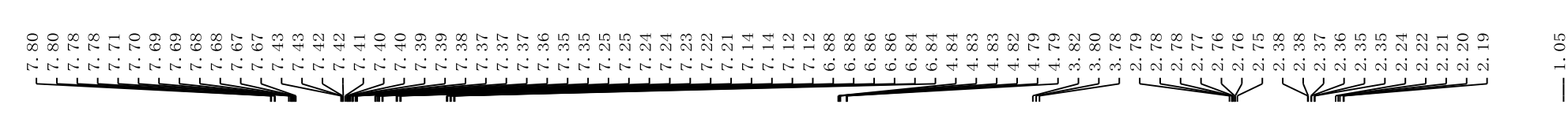

O'pomen

Whis

114

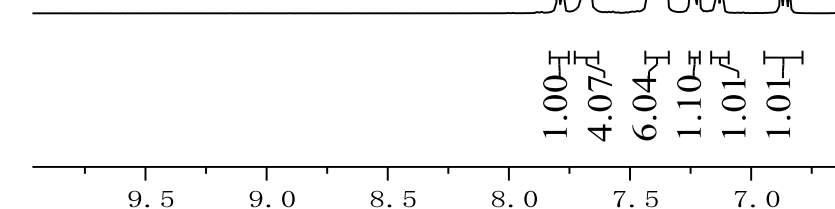

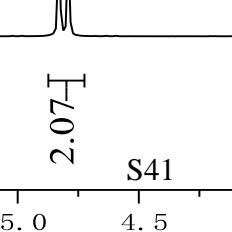




$$
\text { لنلبلت }
$$



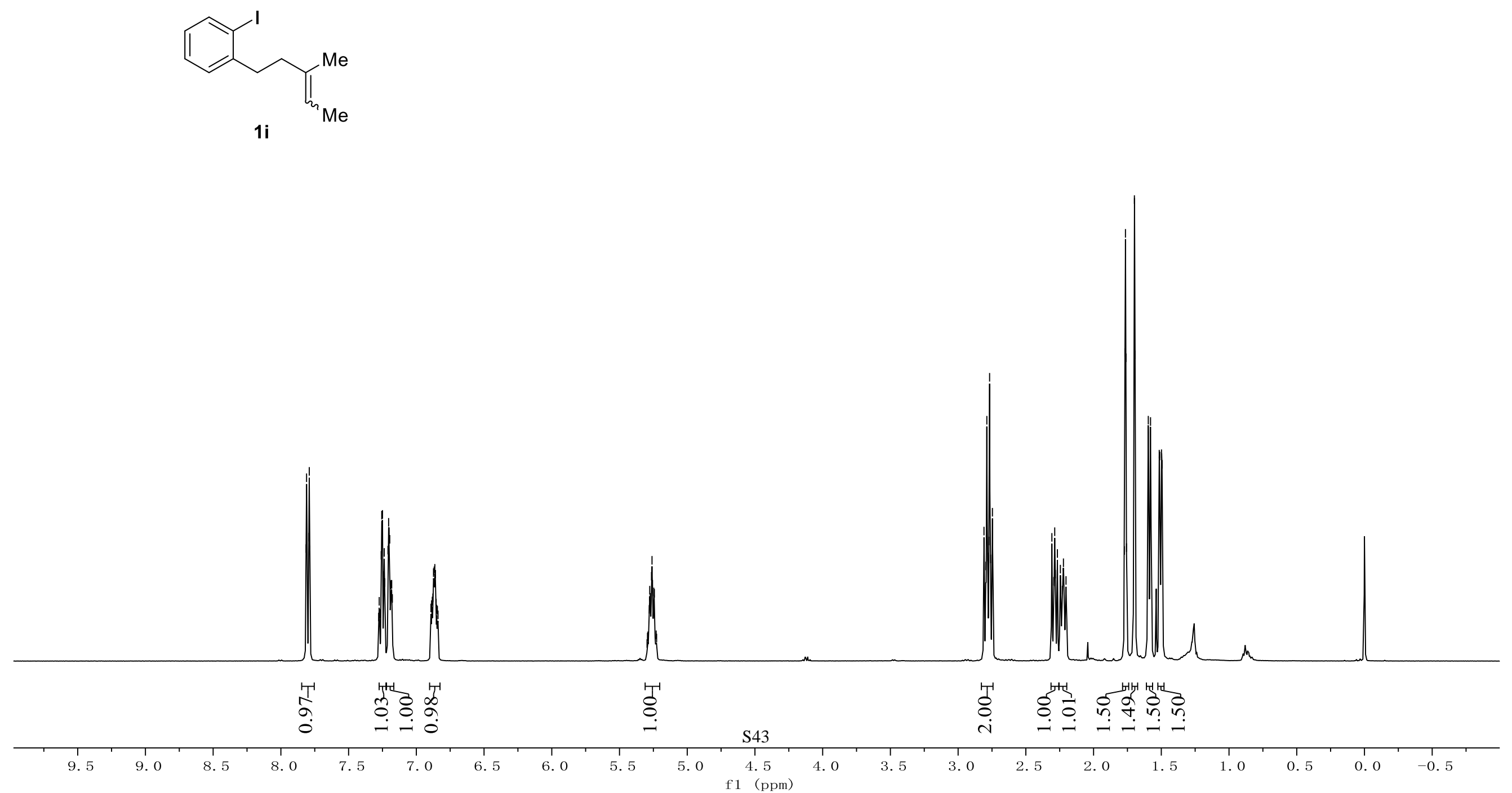


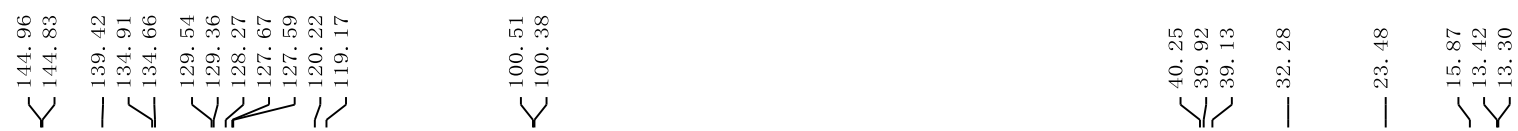
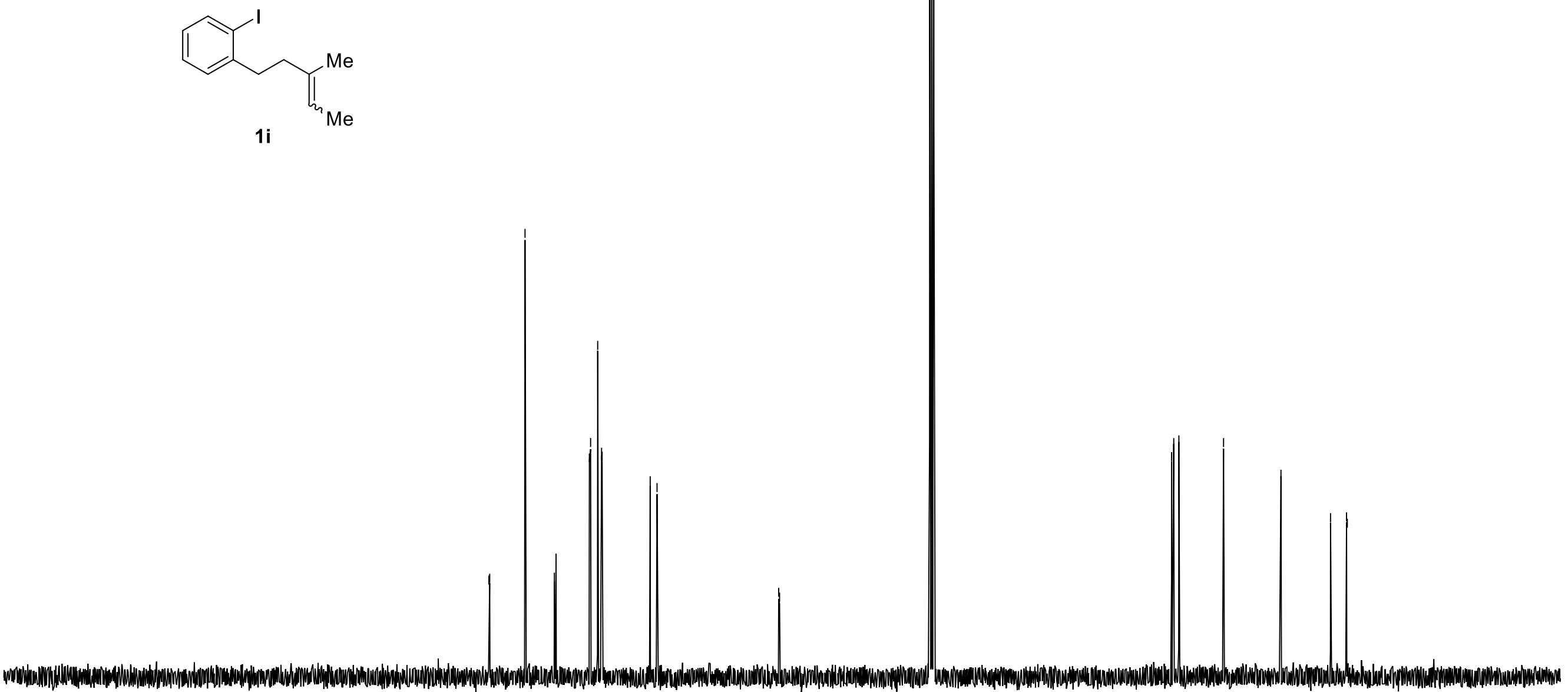

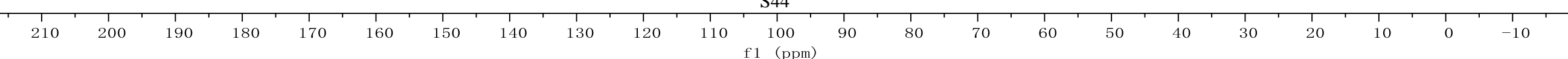




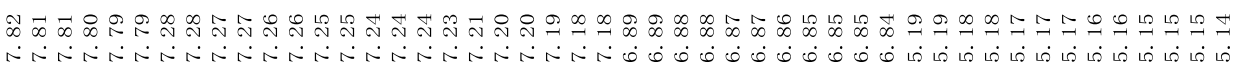

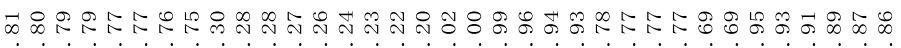

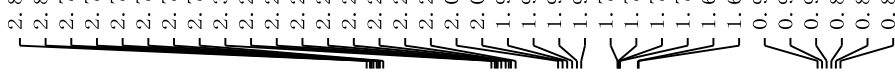<smiles>CCC=C(C)CCc1ccccc1I</smiles>

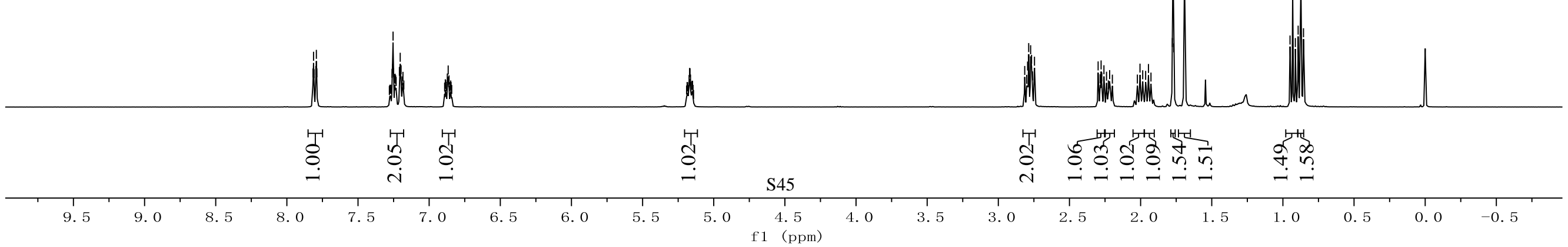




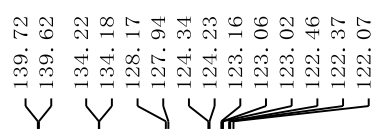<smiles>CCC=C(C)CCc1ccccc1I</smiles>

1j

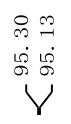

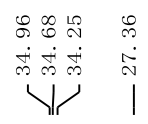

సু $\stackrel{\infty}{\sim}$
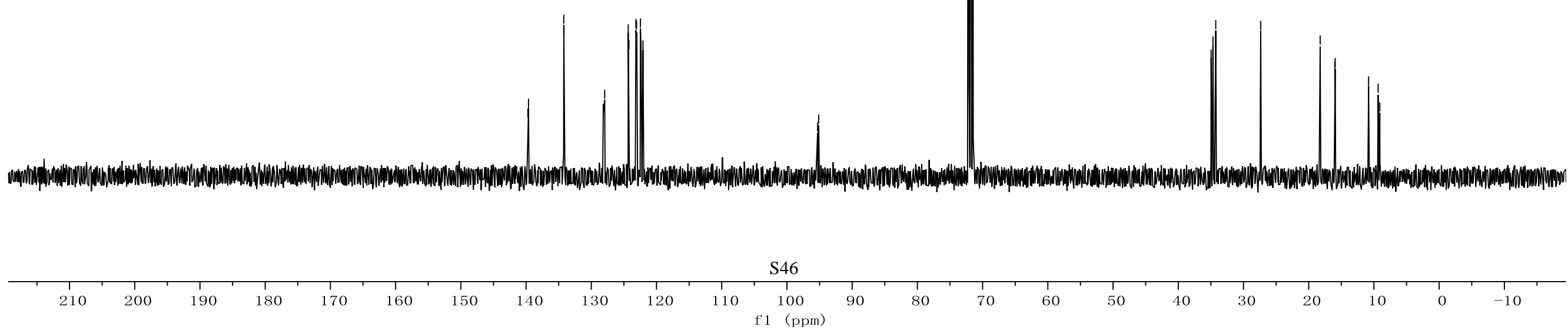


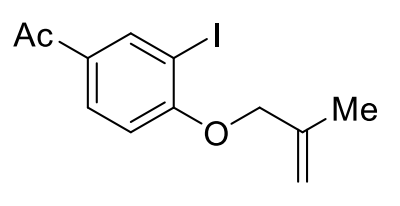

10

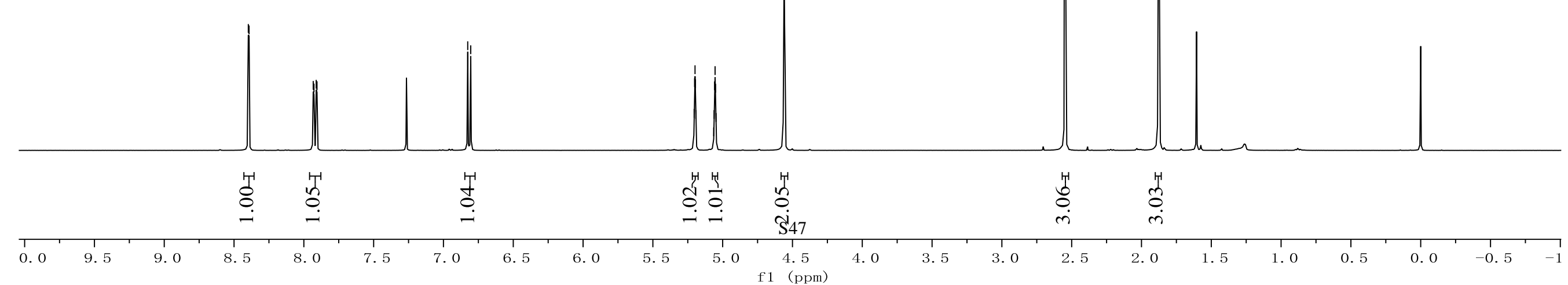




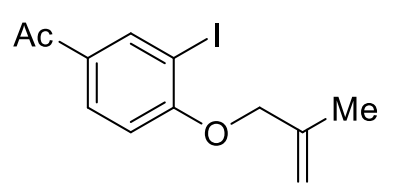

10

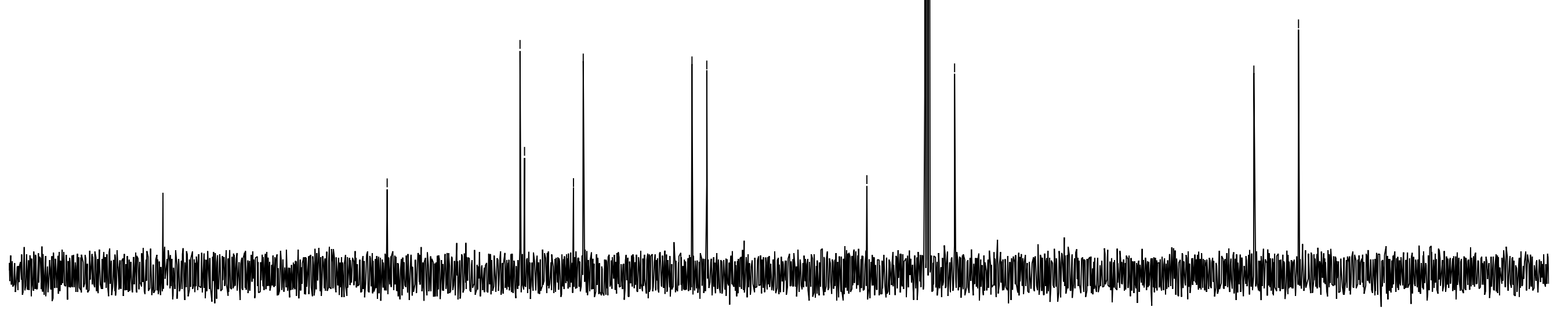

S48
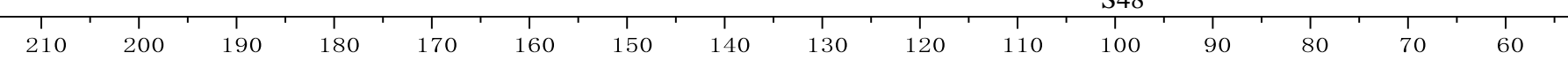

50

30

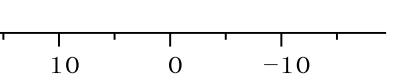



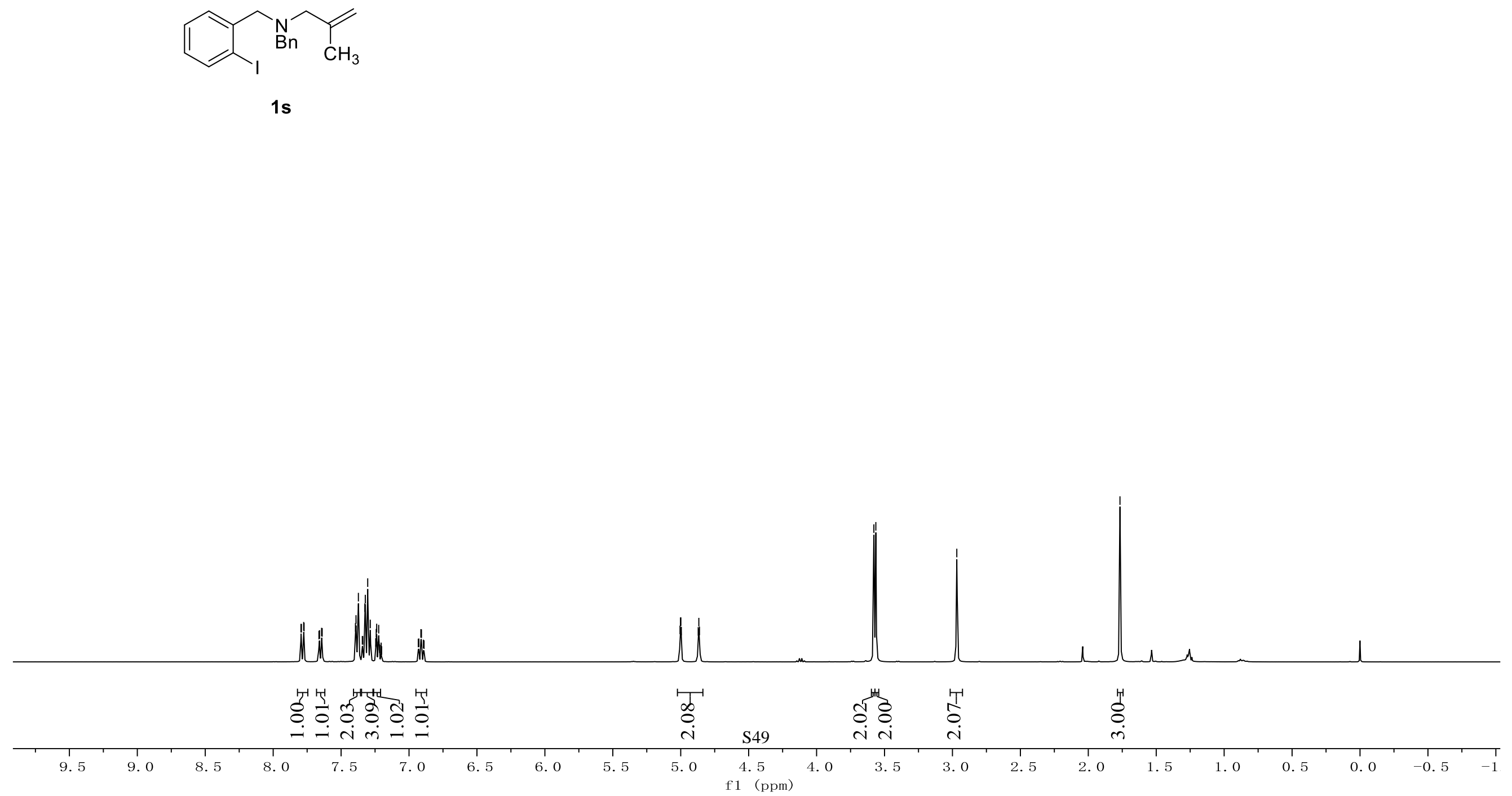


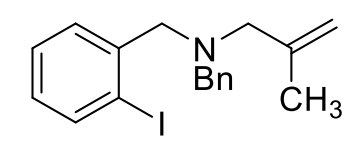

$1 \mathrm{~s}$
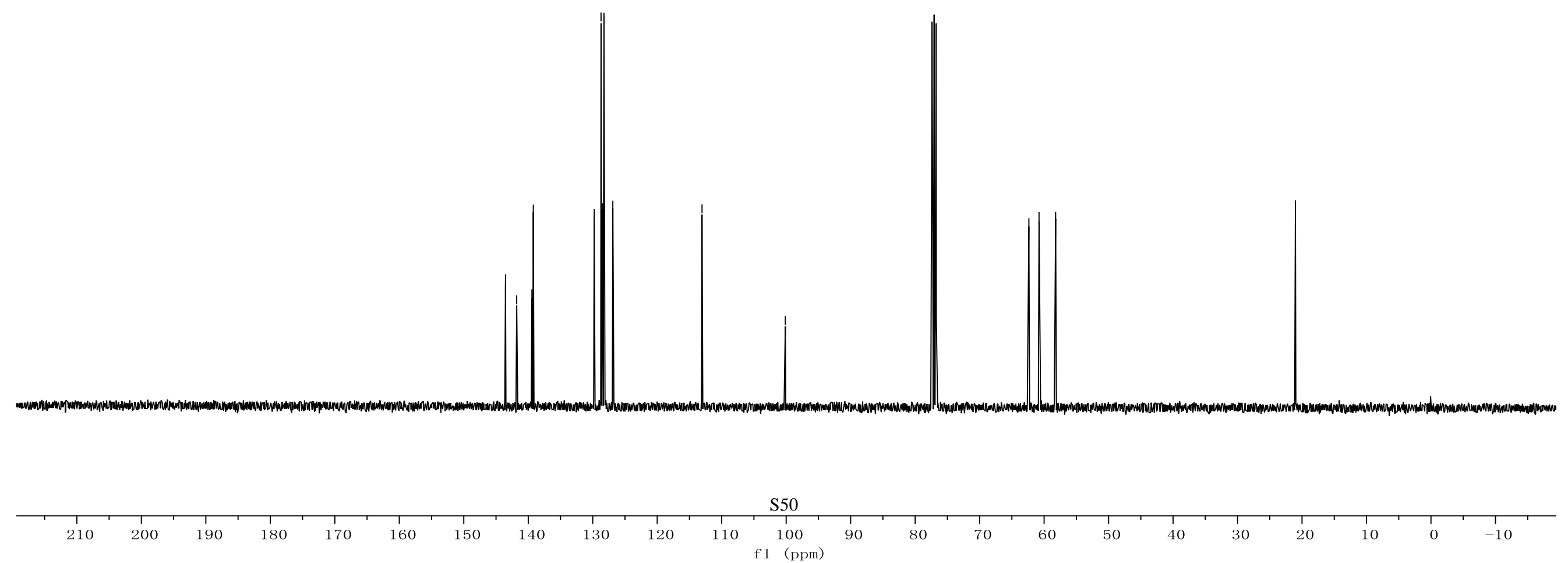

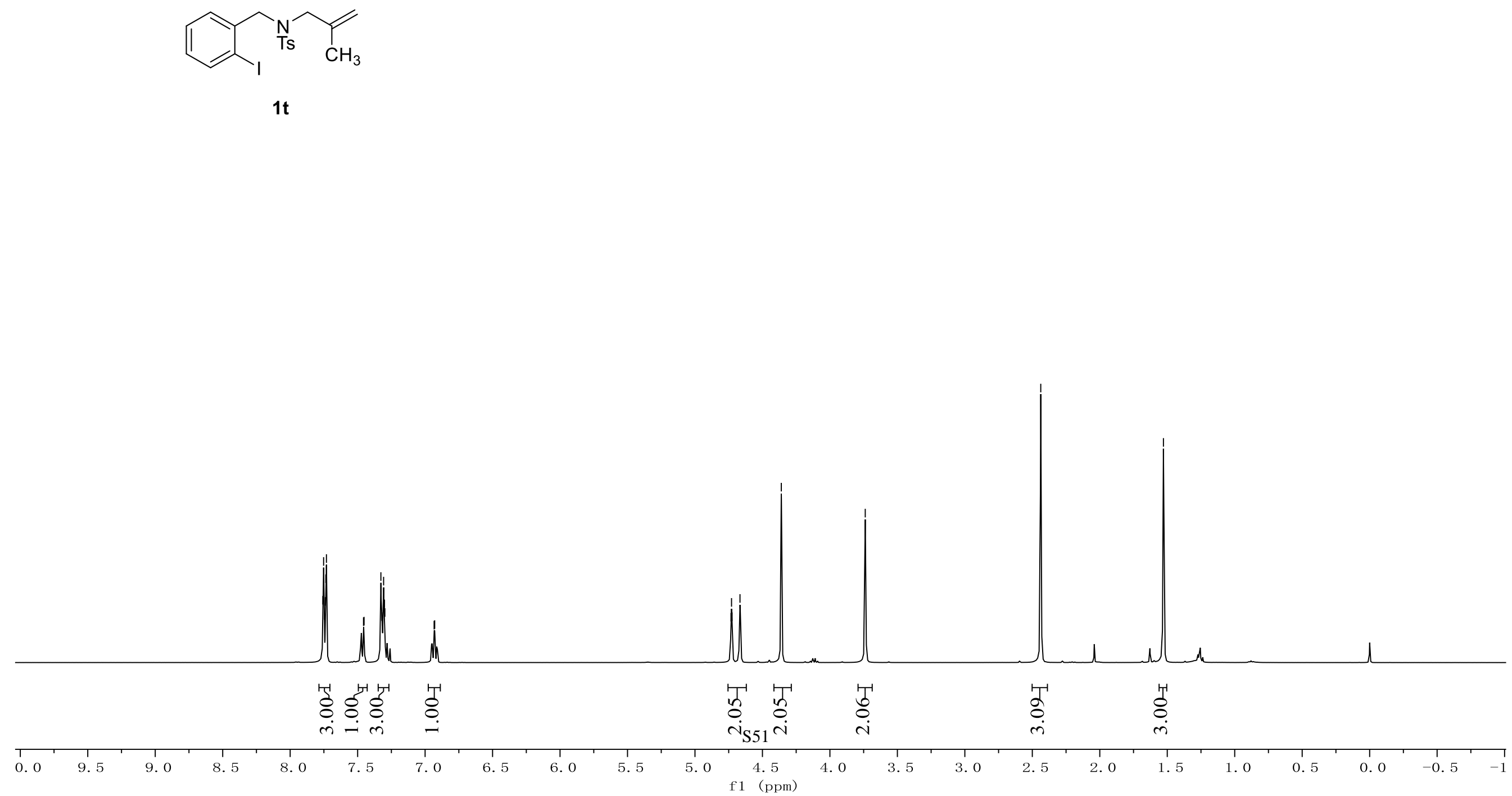

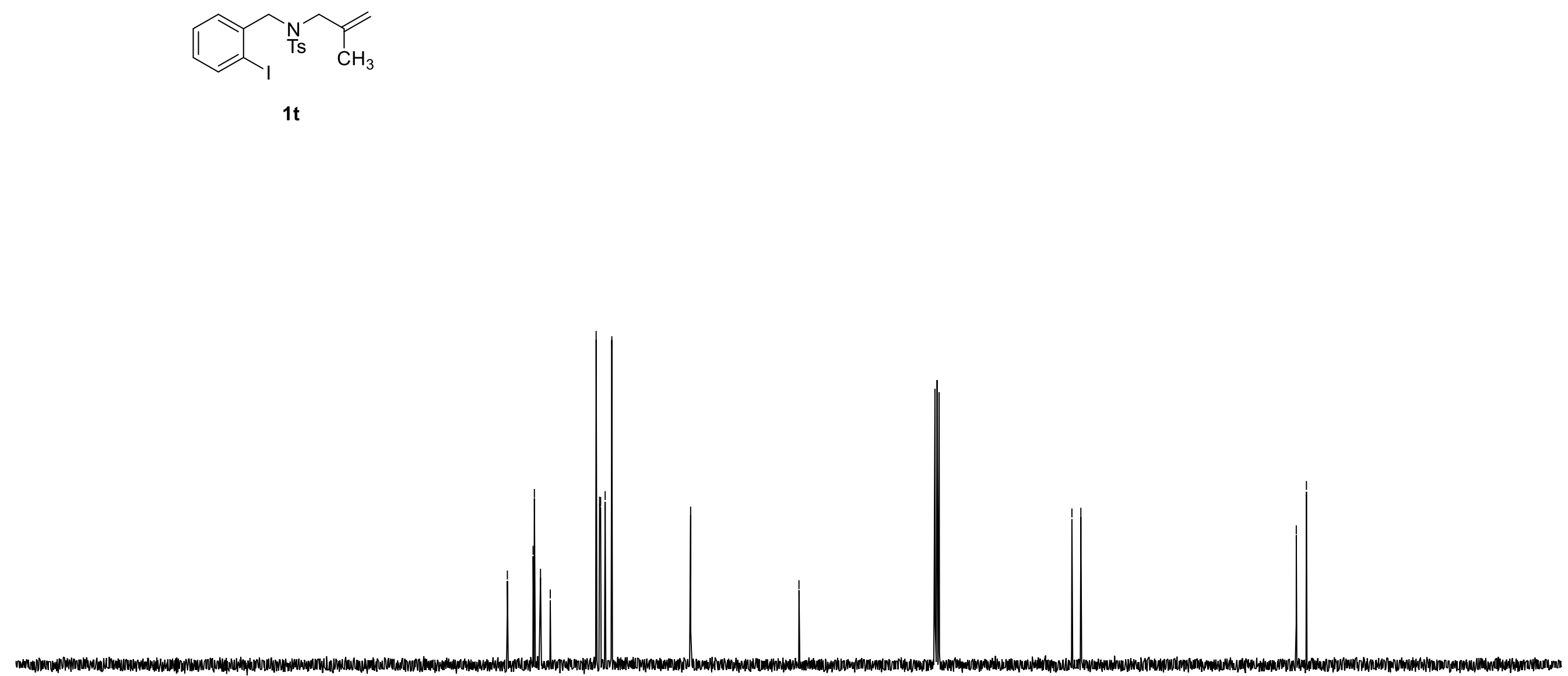

S52

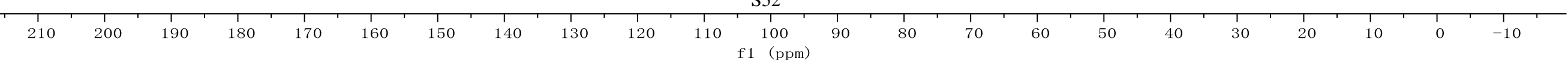




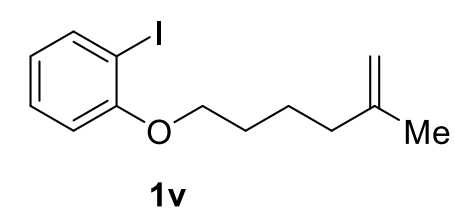

$1 v$ 


$$
\text { w }
$$



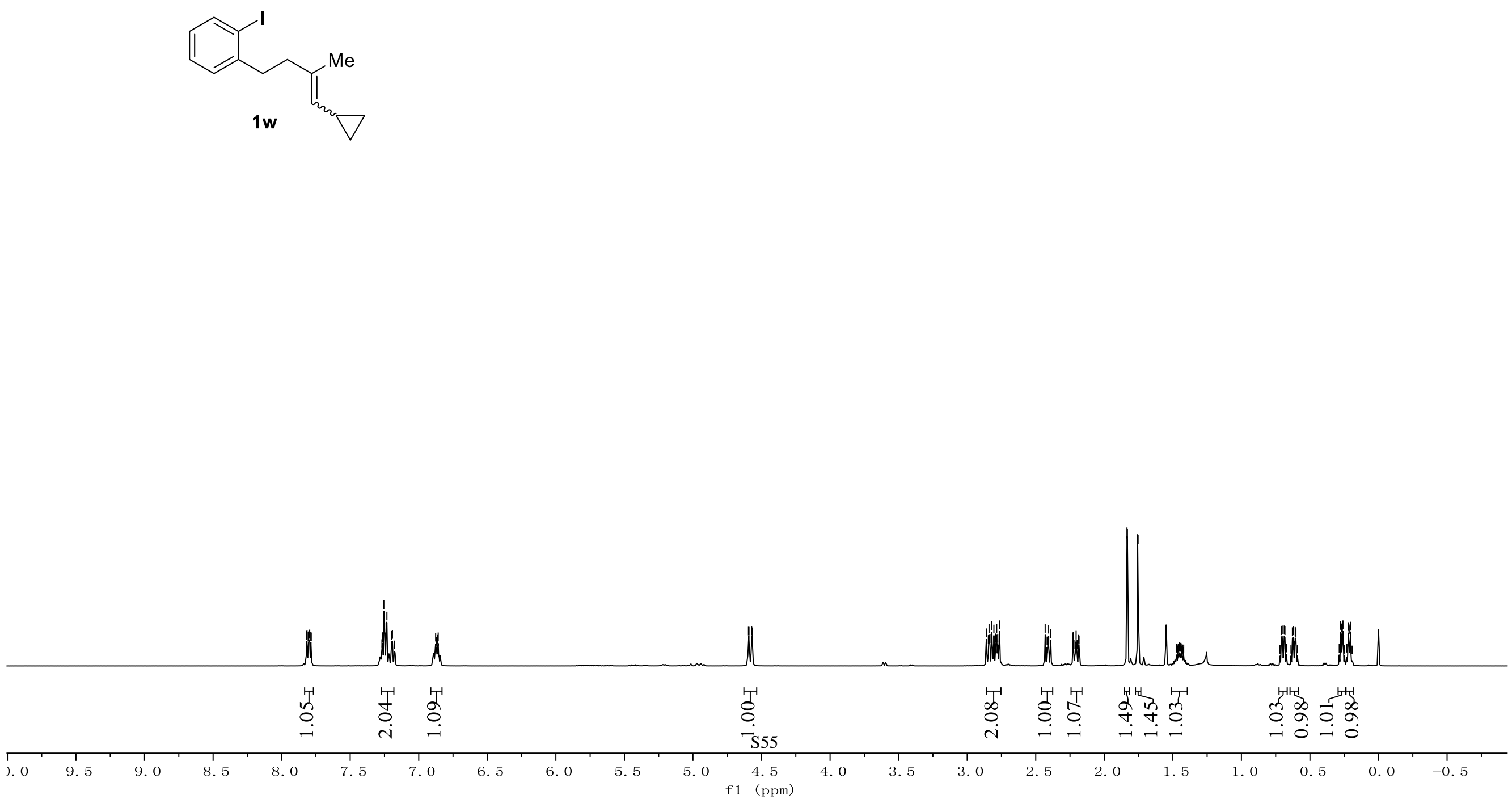

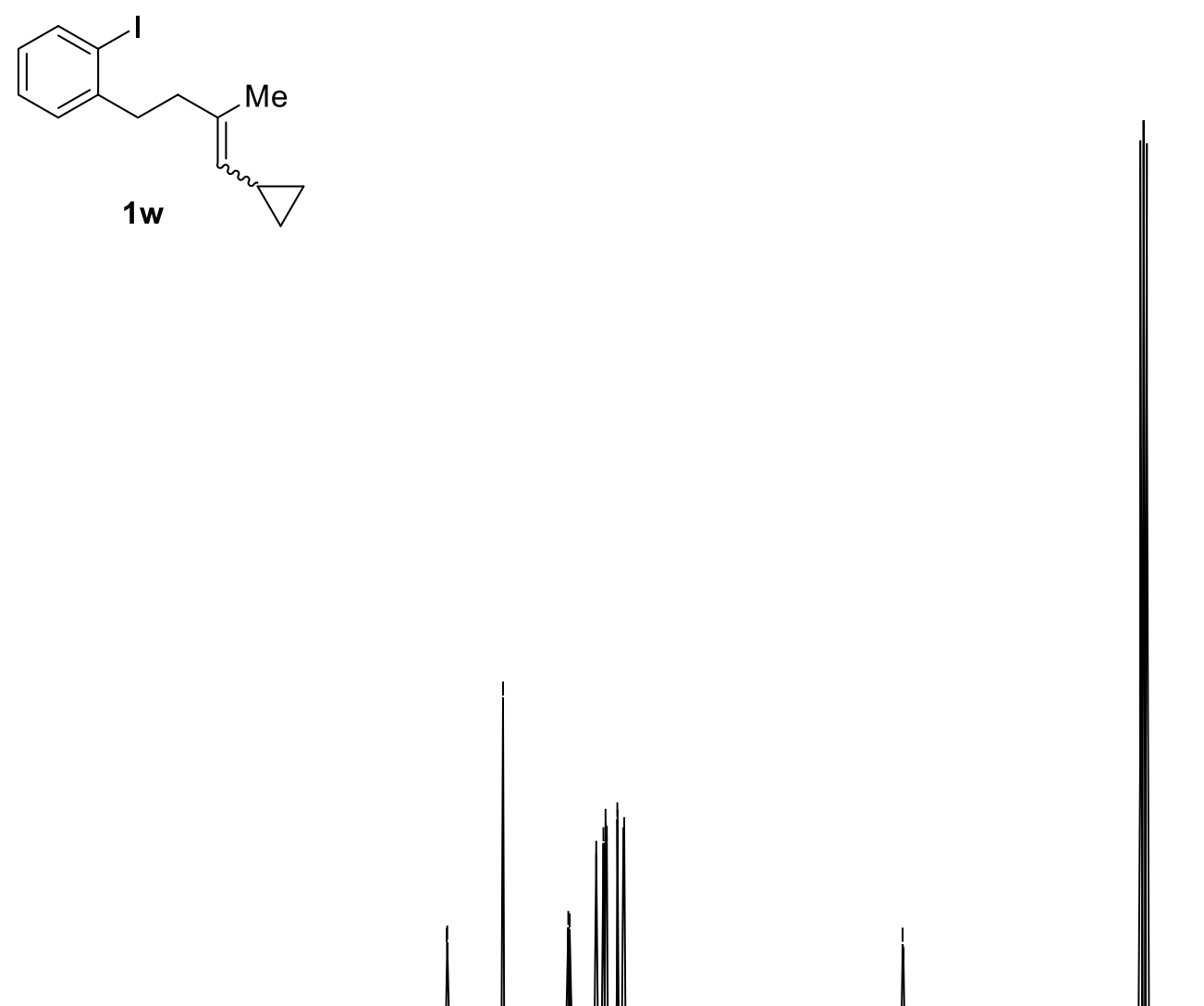

S56

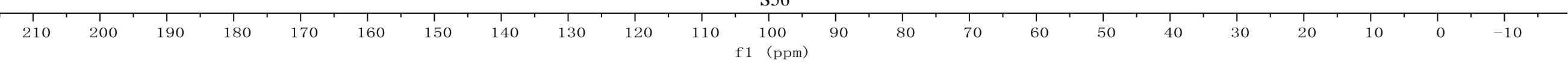




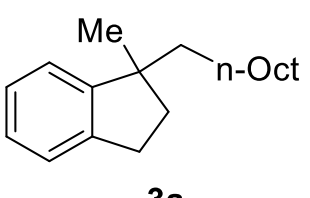

3a

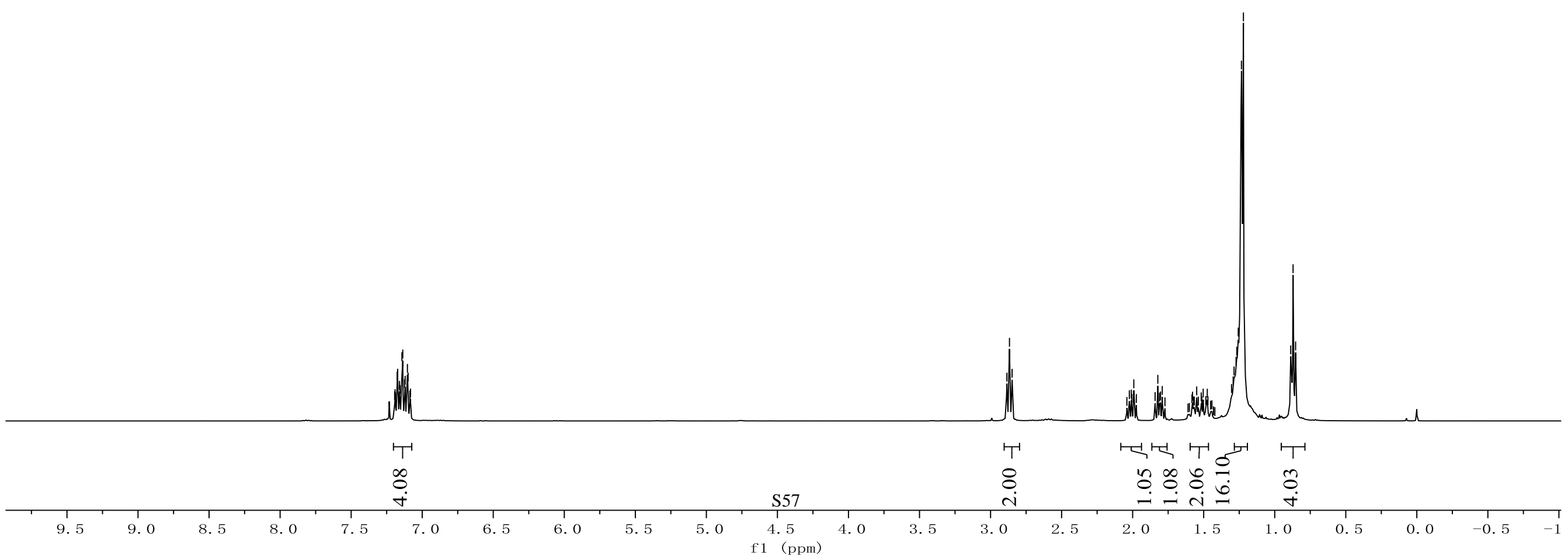




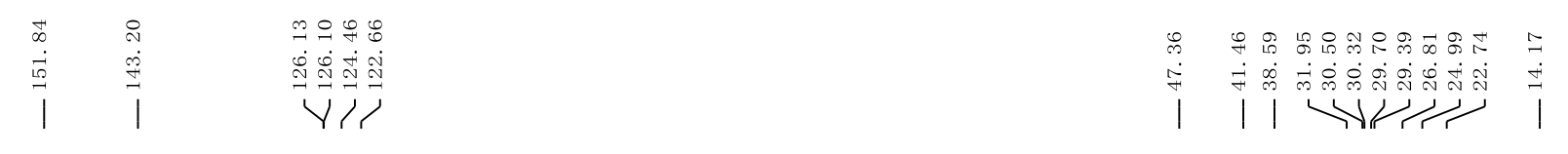

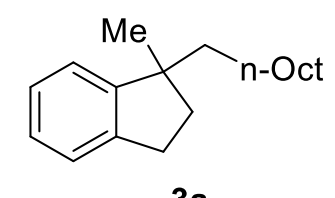

$3 a$
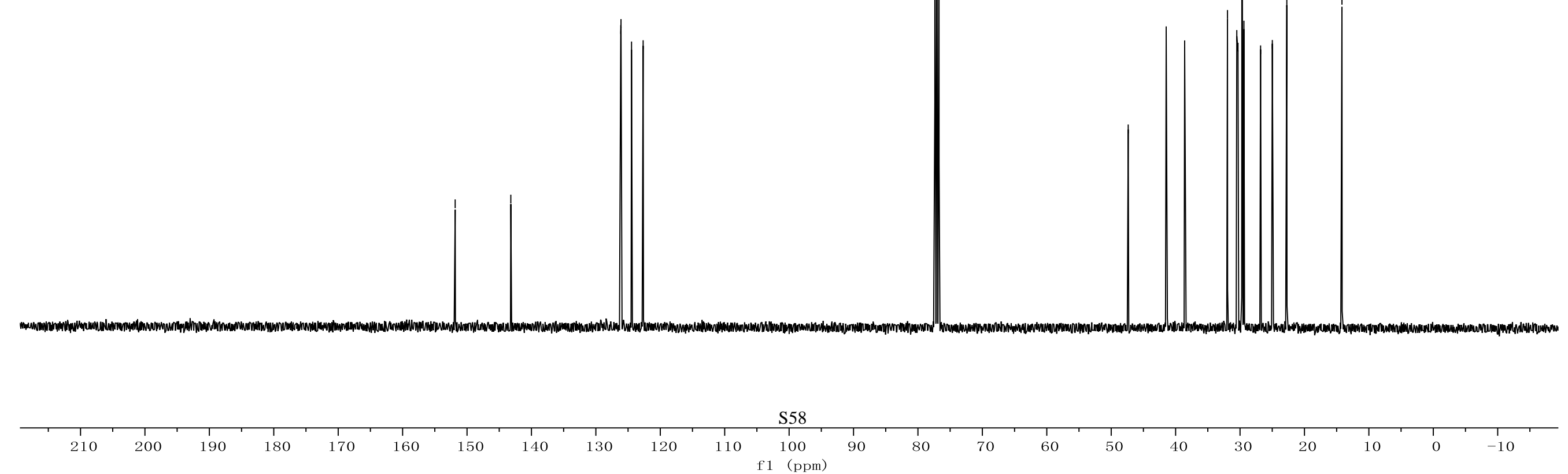


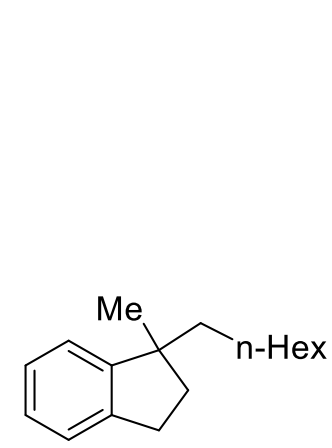

3b

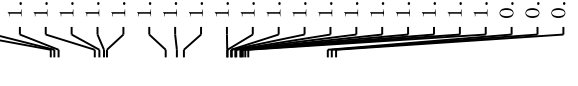




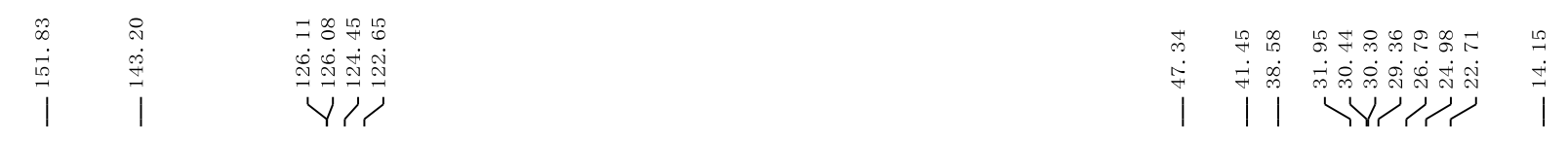

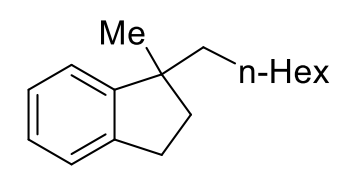

3b
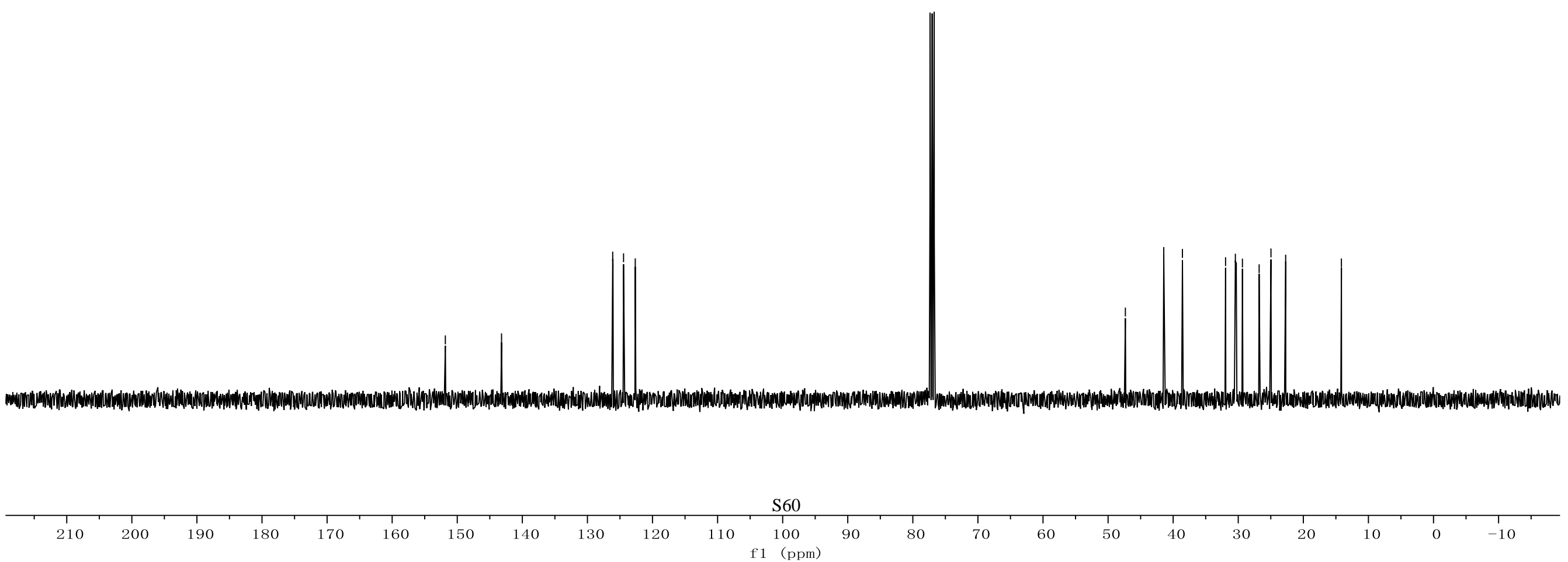


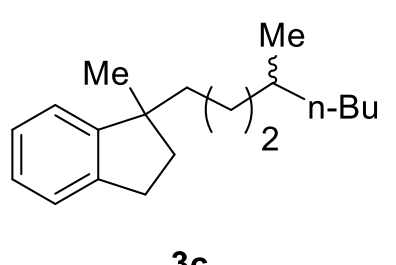

$3 c$

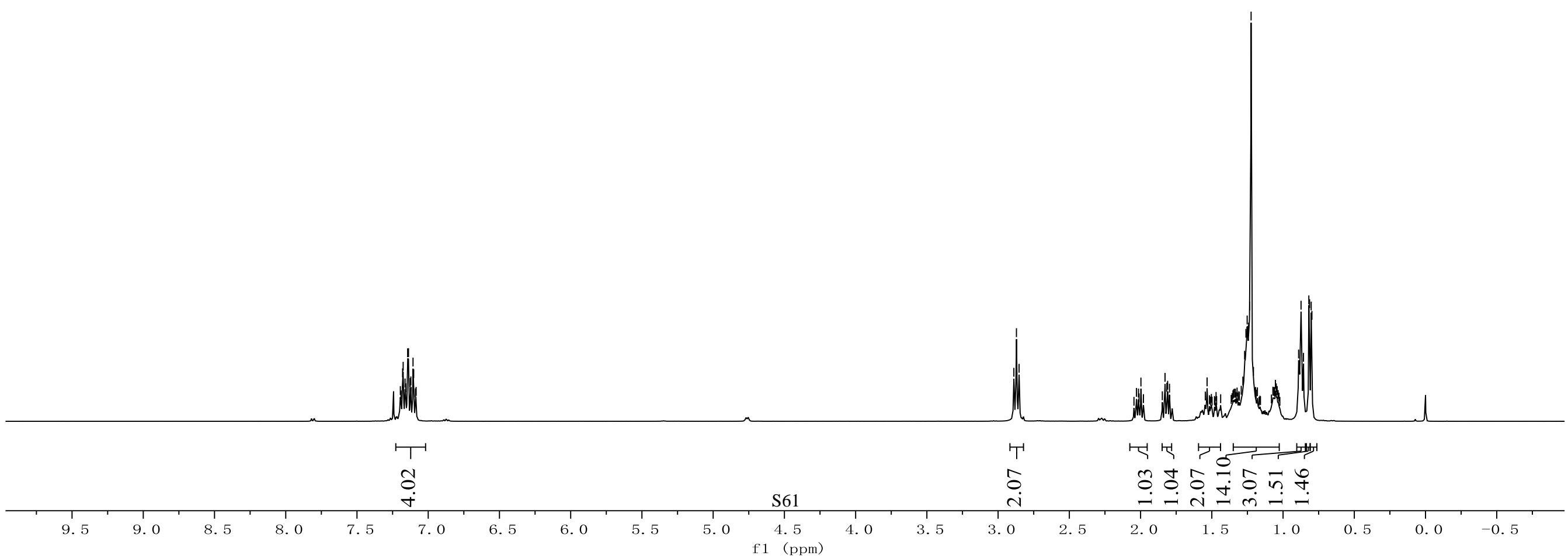




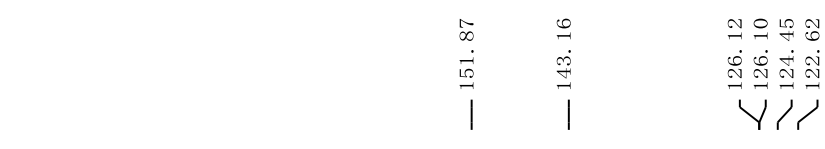

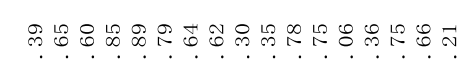

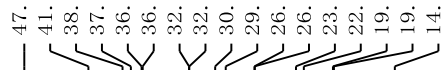

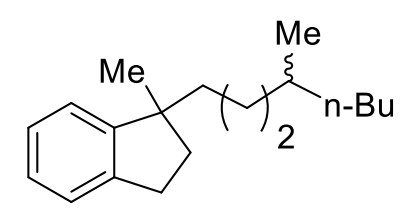

$3 c$

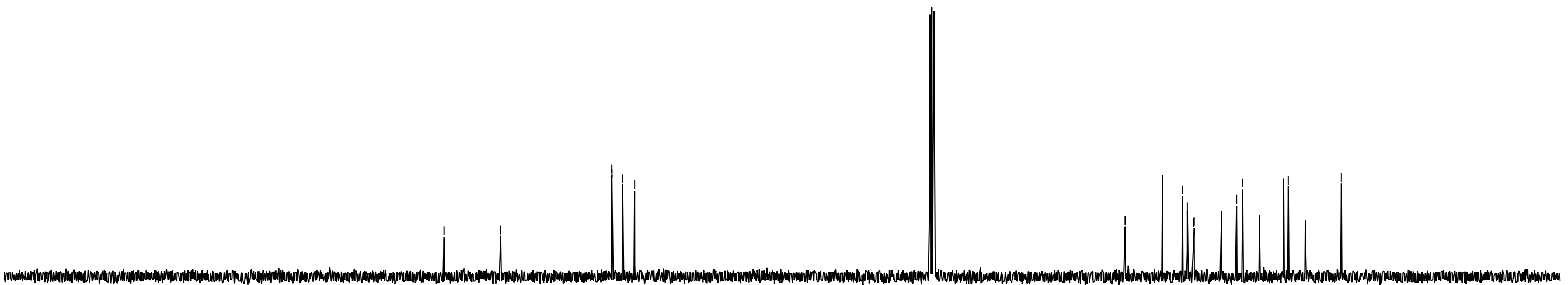

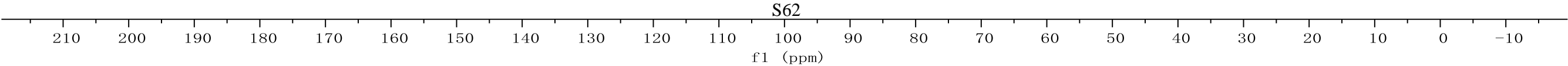




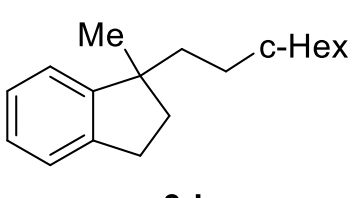

3d

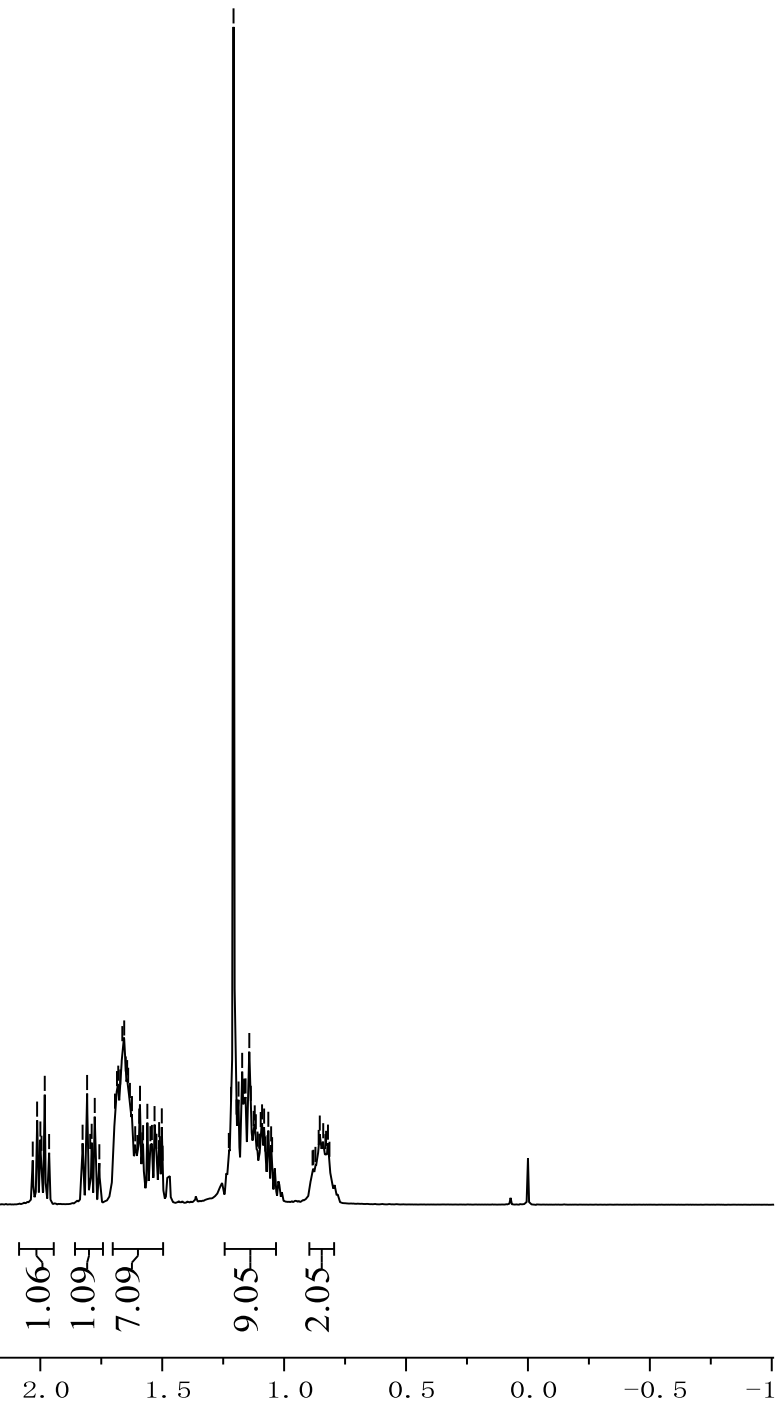




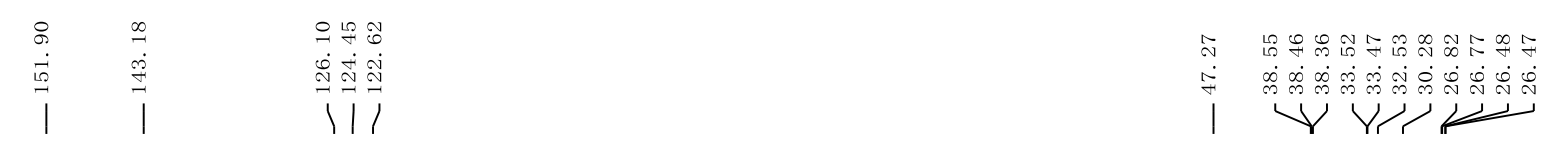
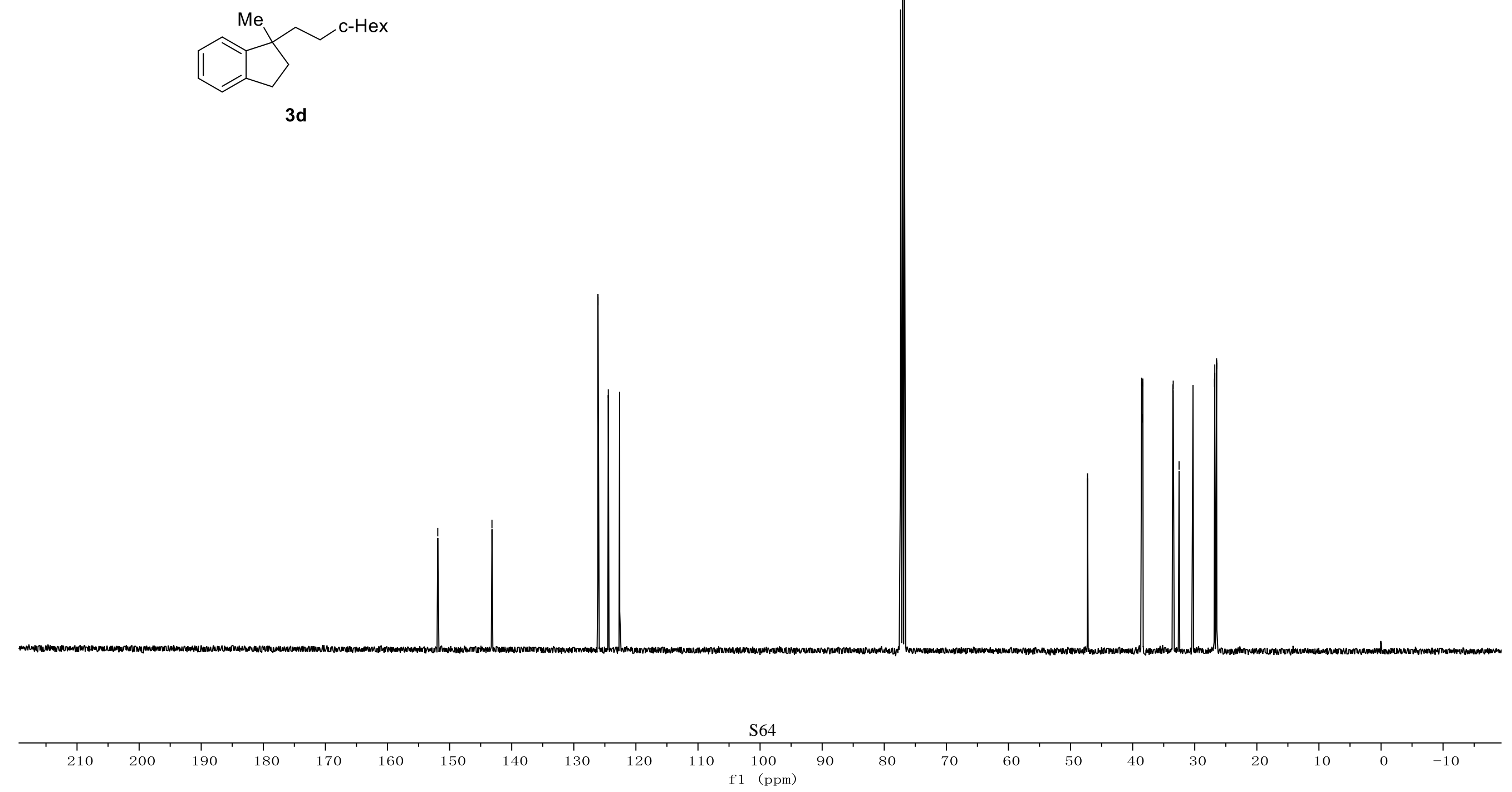


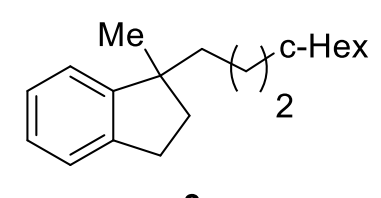

$3 e$

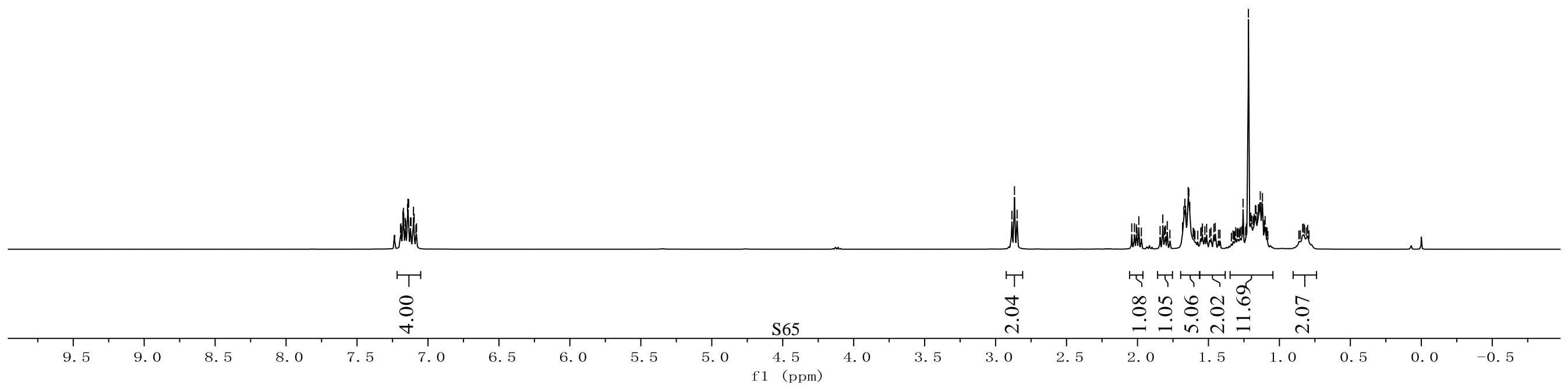



$\infty \quad \infty \quad \cong ㅇ ㅠ$

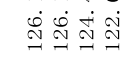

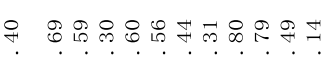
苛

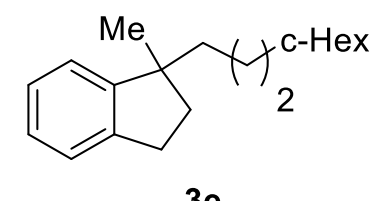

$3 e$
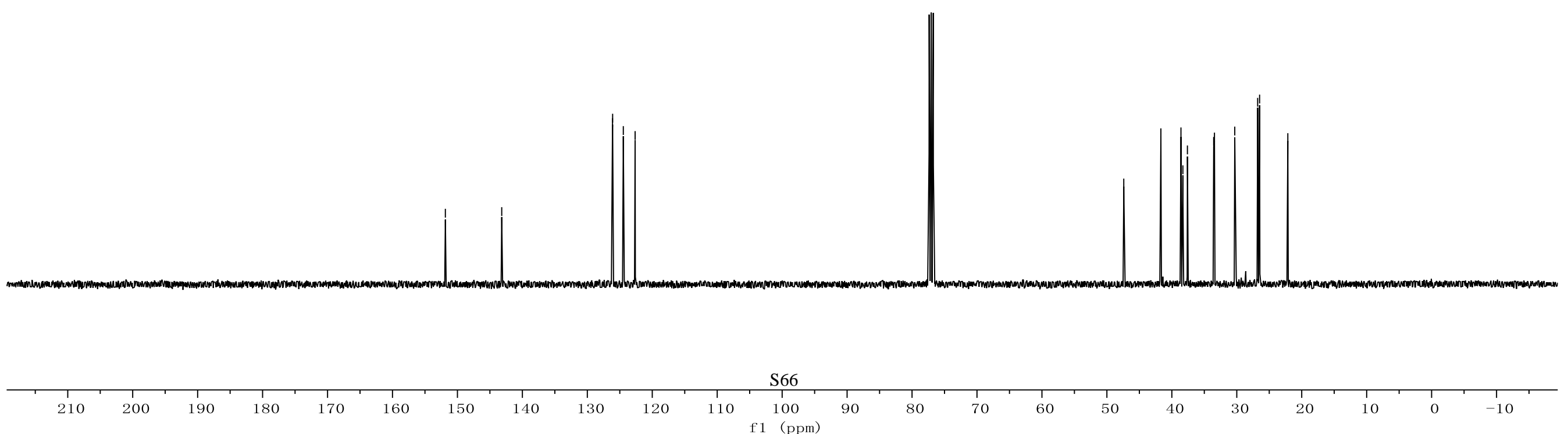

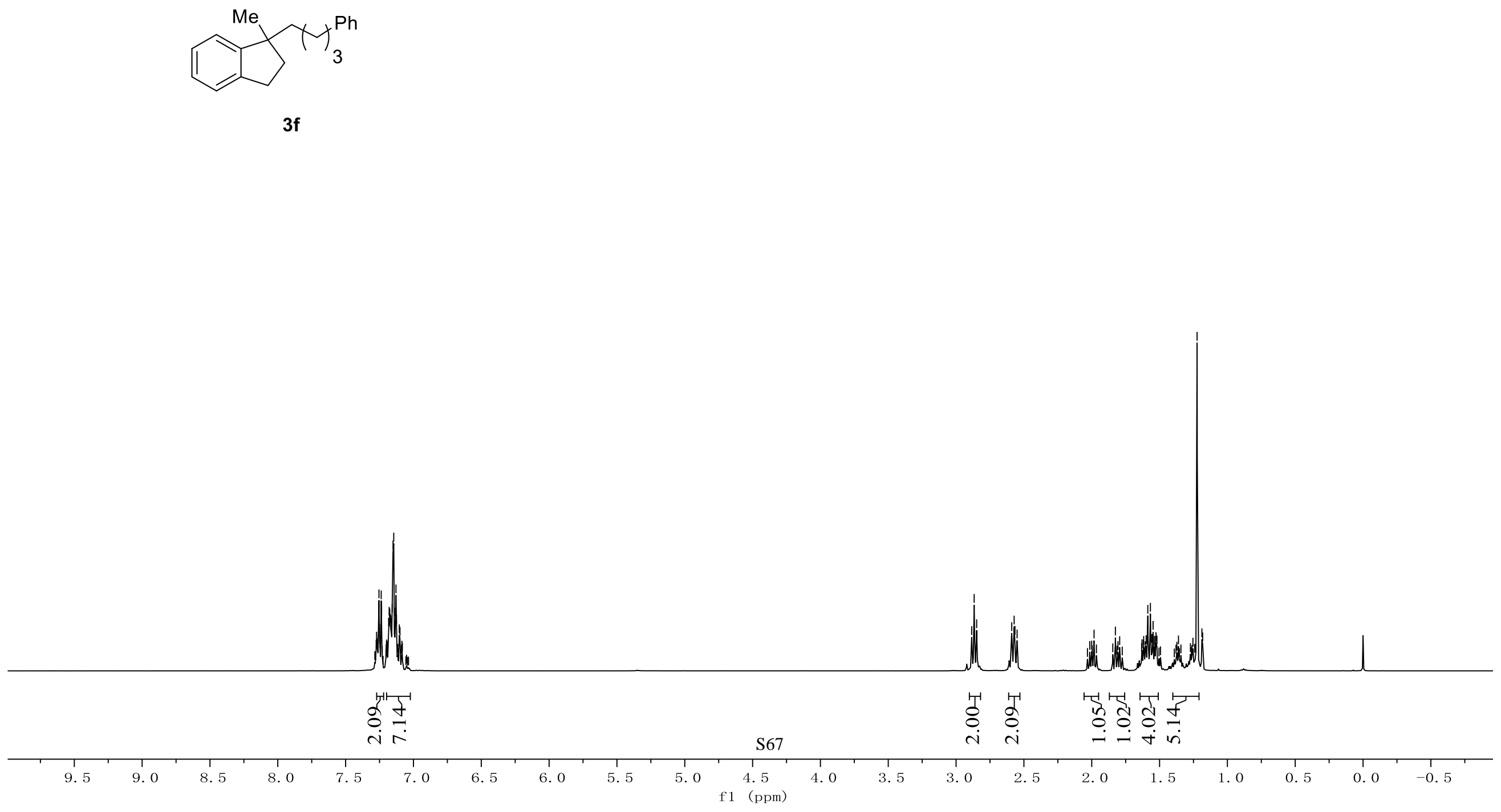


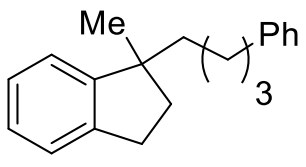

$3 f$

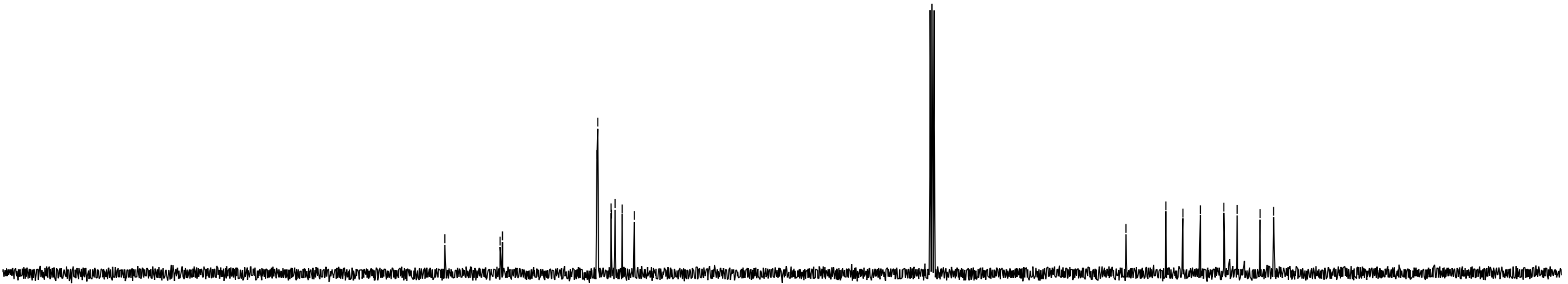

S68

\begin{tabular}{|c|c|c|c|c|c|c|c|c|c|c|}
\hline 1 & 1 & 190 & 180 & 170 & 1 & 1 & 1 & 1 & 1 & 110 \\
\hline
\end{tabular}
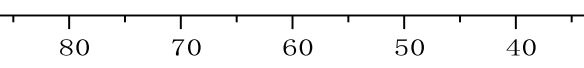

30

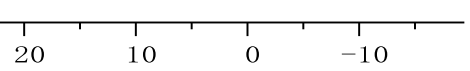




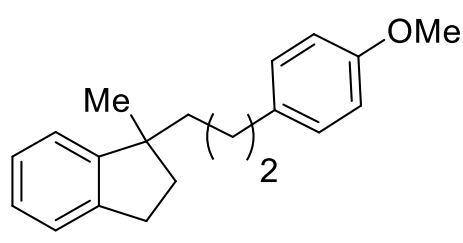

$3 g$

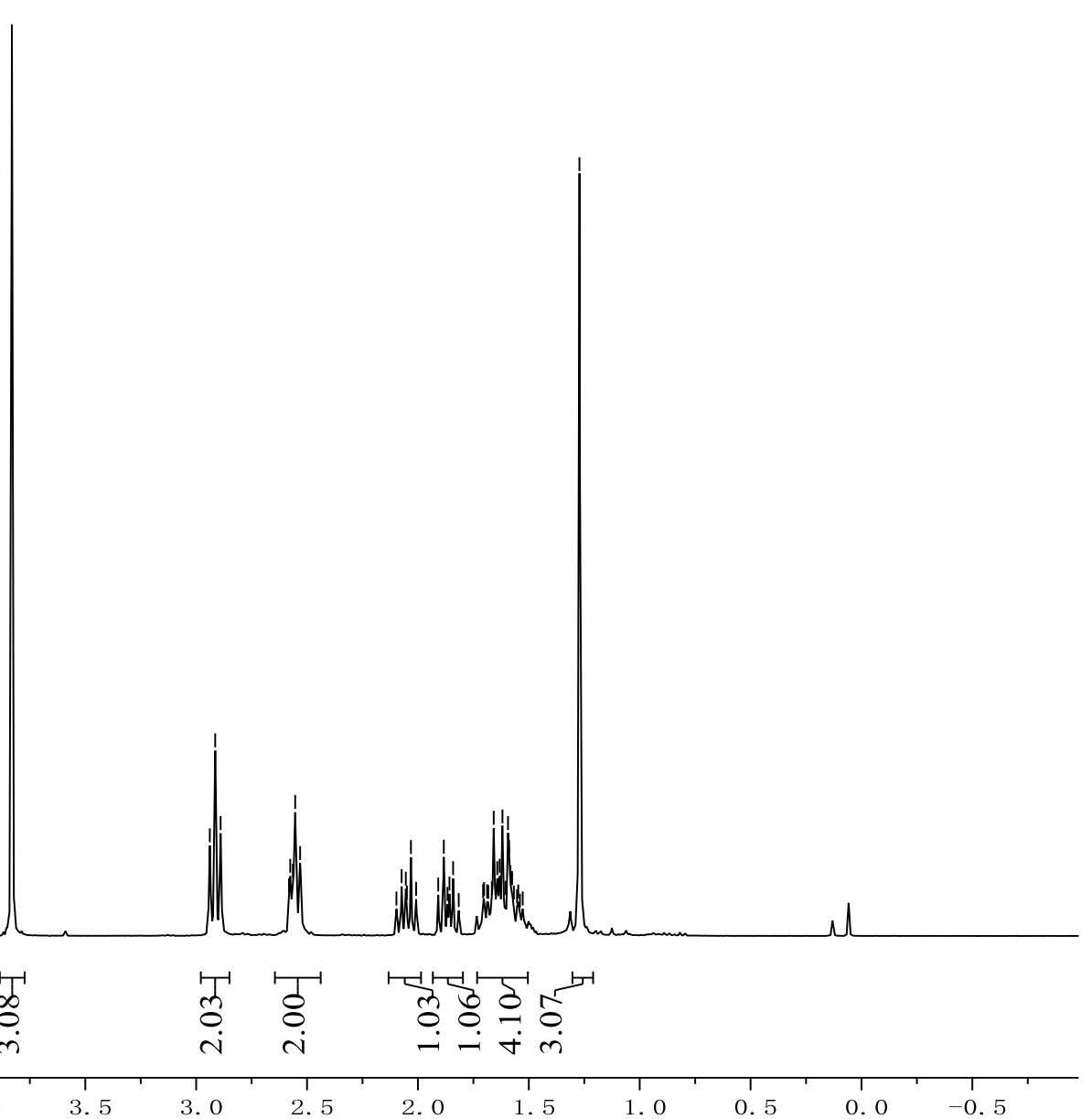




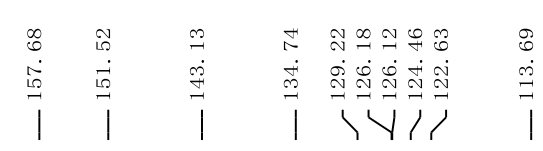

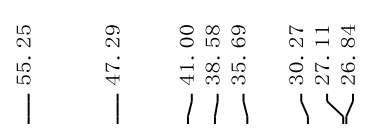

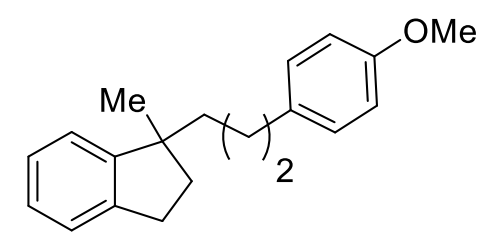

$3 g$
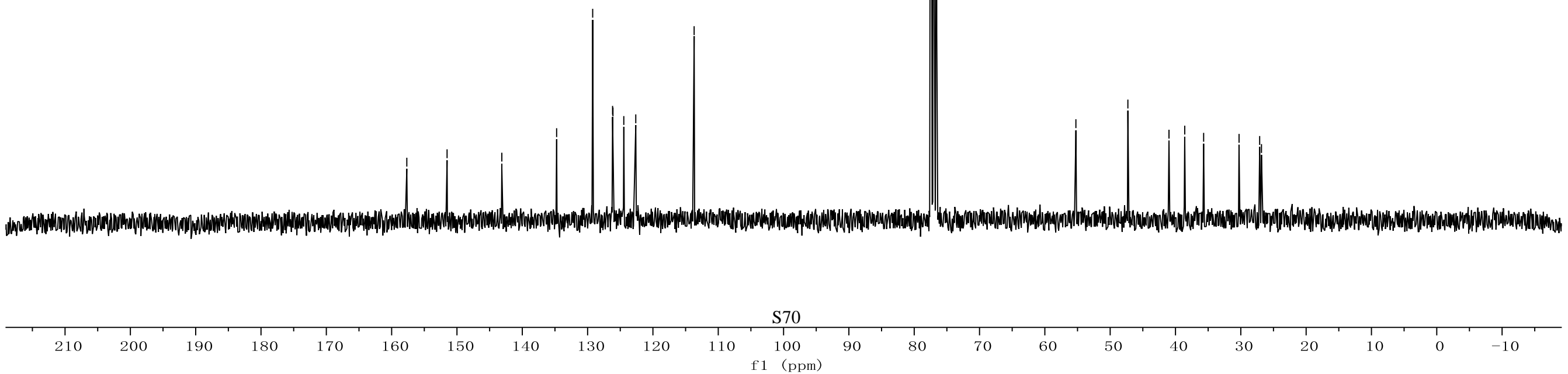


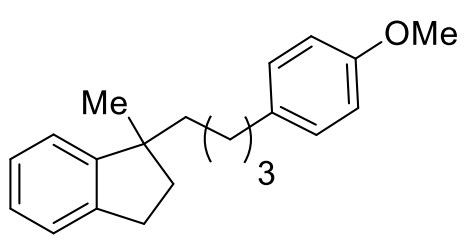

$3 \mathrm{~h}$

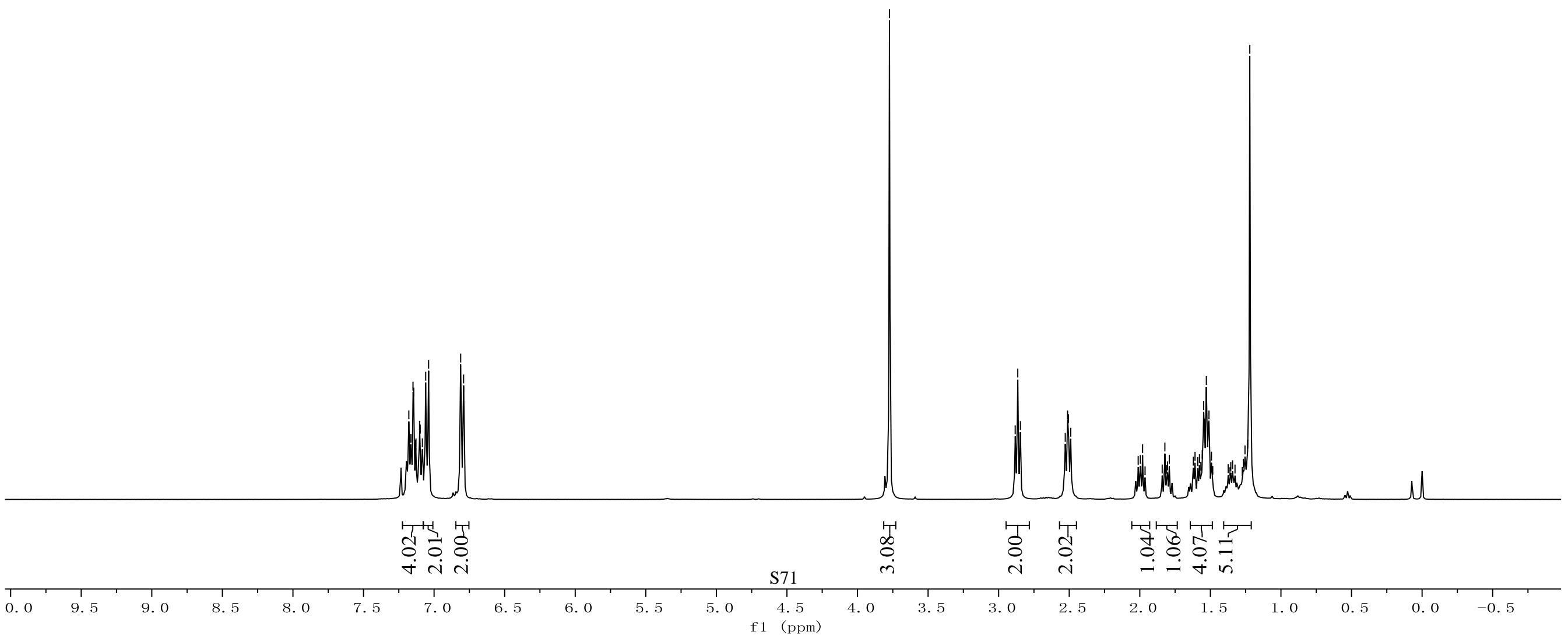




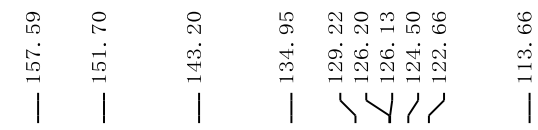

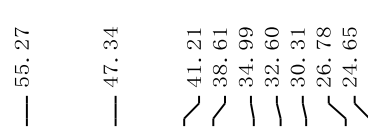

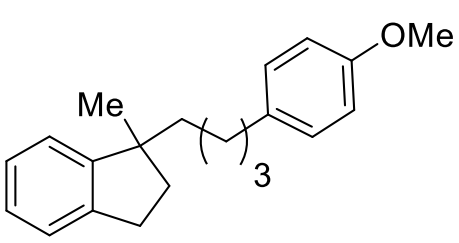

3h
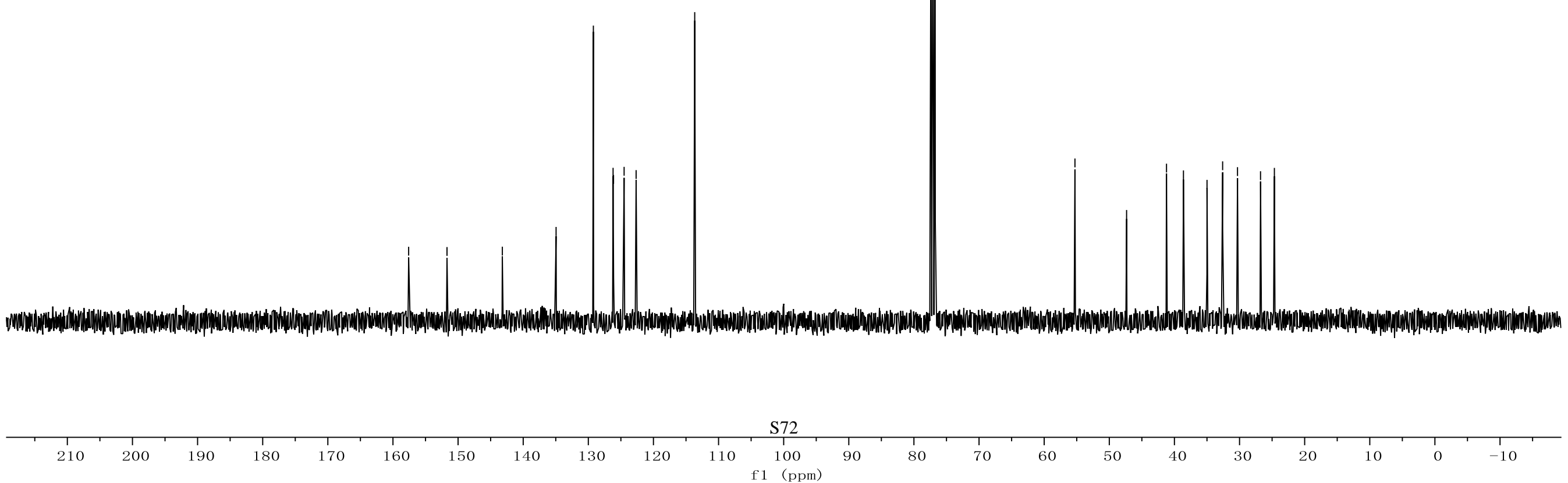


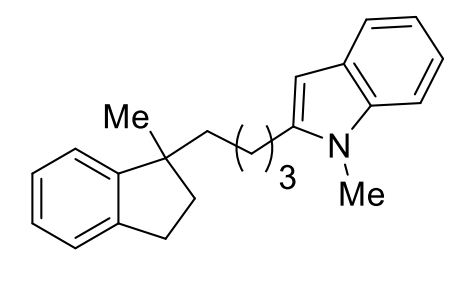

$3 i$

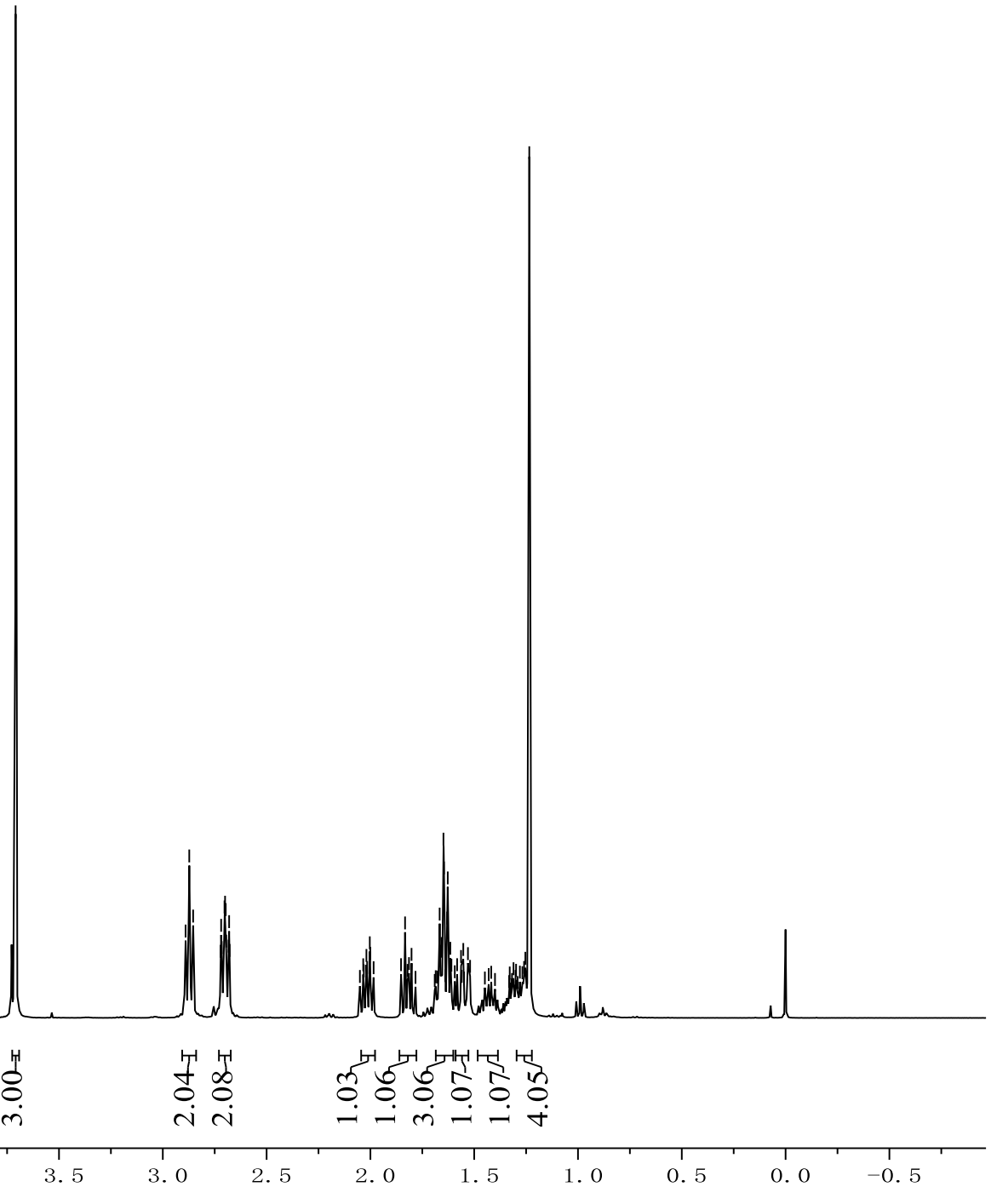




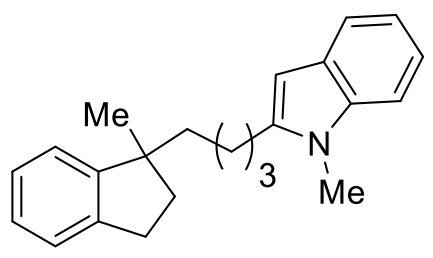

$3 \mathbf{i}$
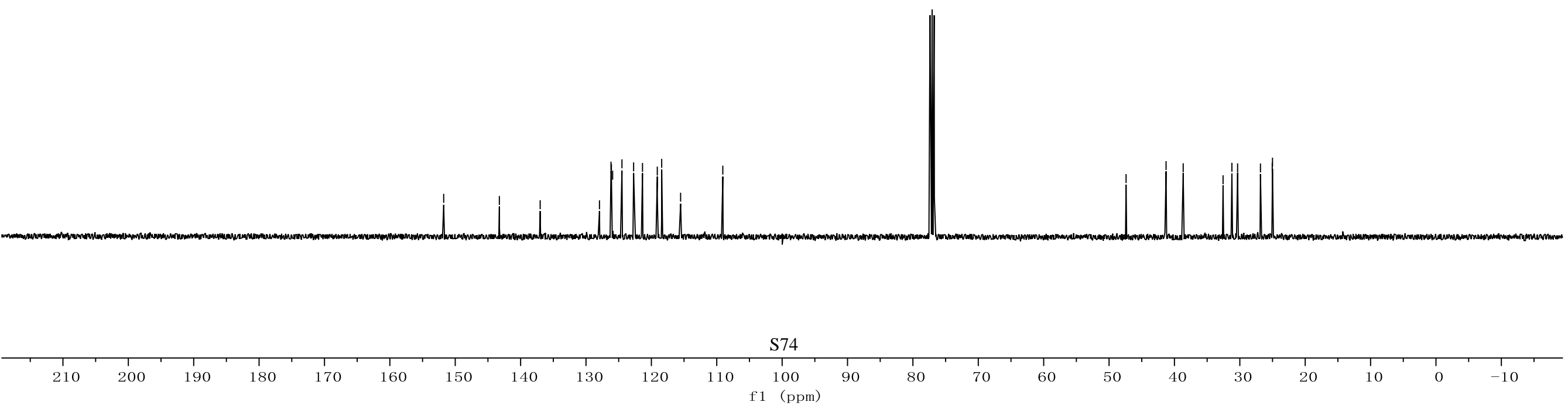
<smiles>CCOC(=O)CCCC1(C)CCc2ccccc21</smiles>

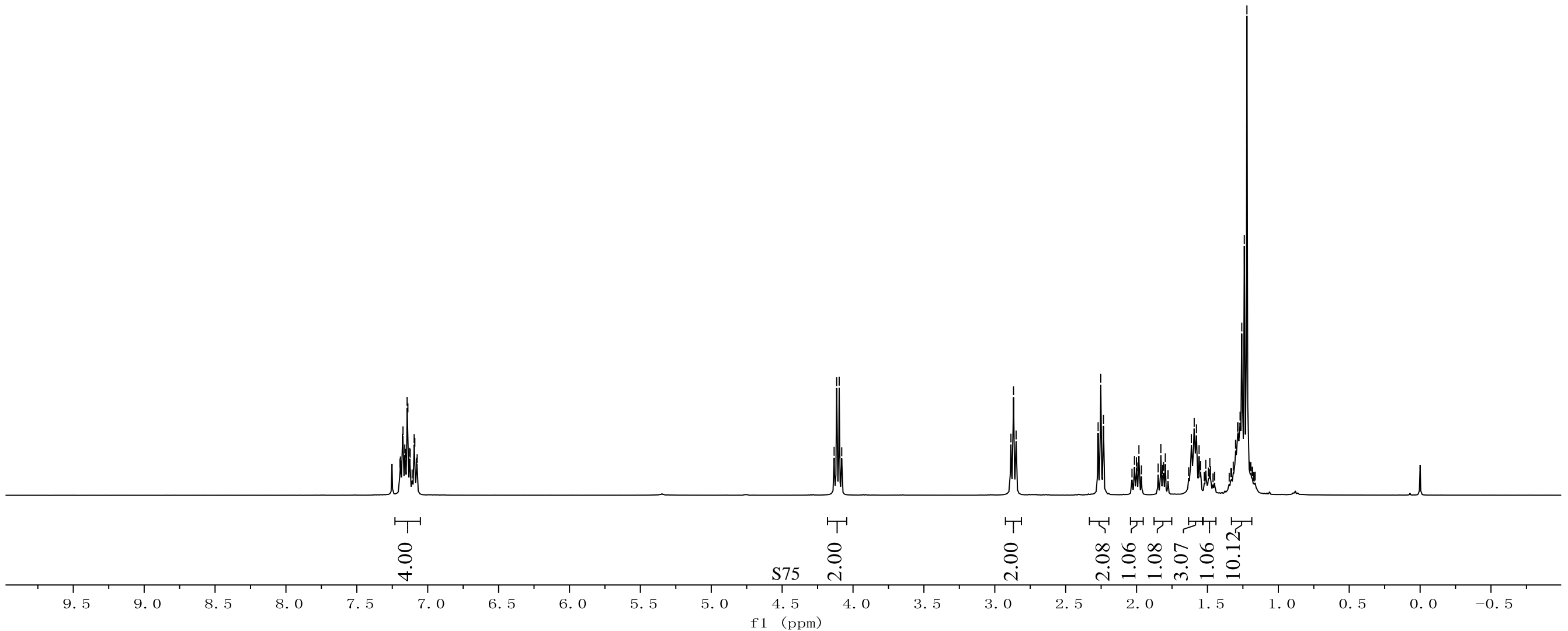




\begin{tabular}{|c|c|c|c|c|}
\hline 㟧 & 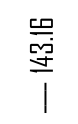 & 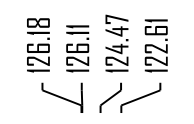 & $\stackrel{\substack{0\\
}}{\mid}$ & 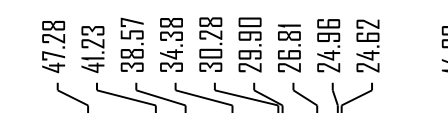 \\
\hline
\end{tabular}
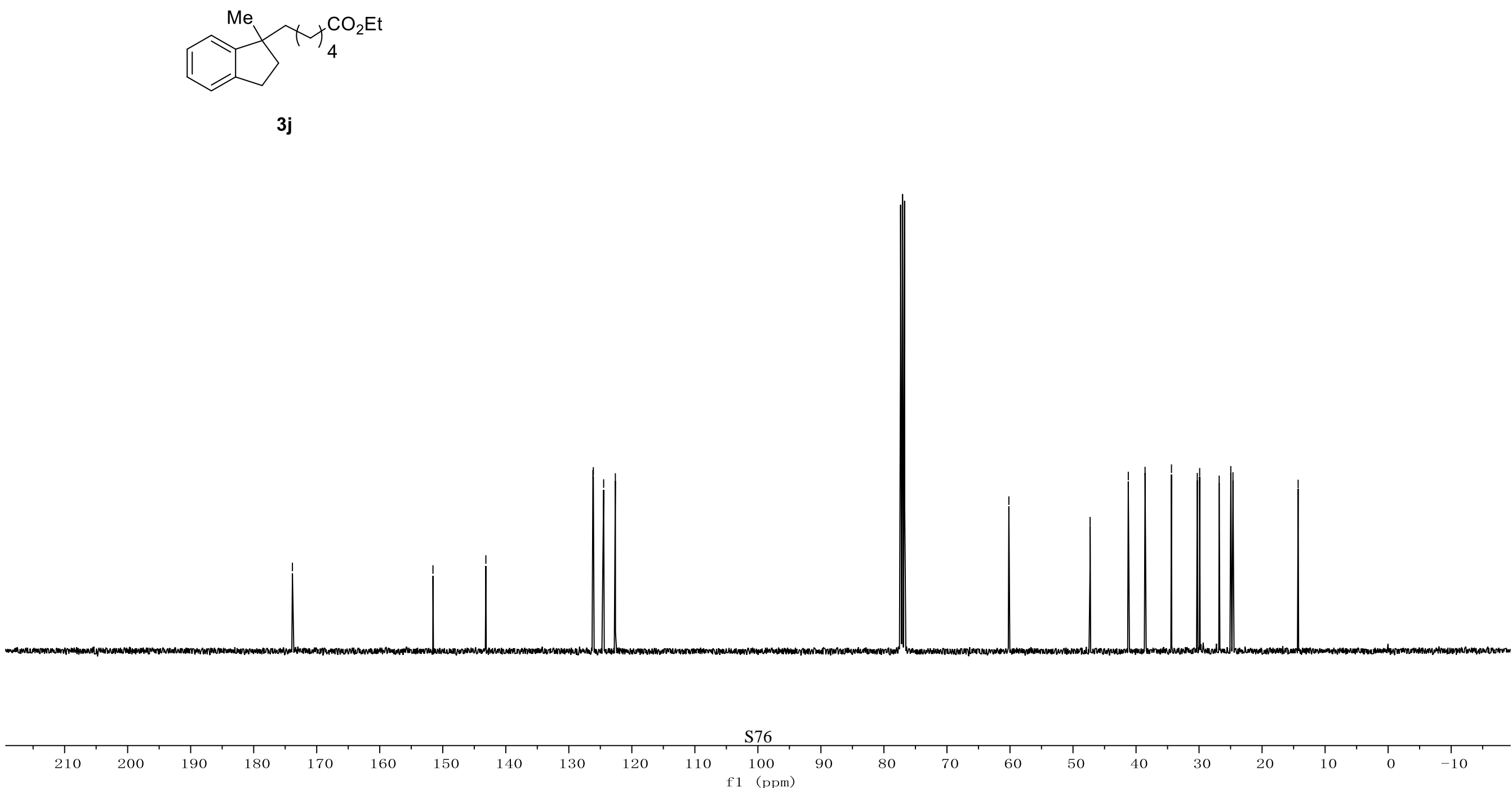

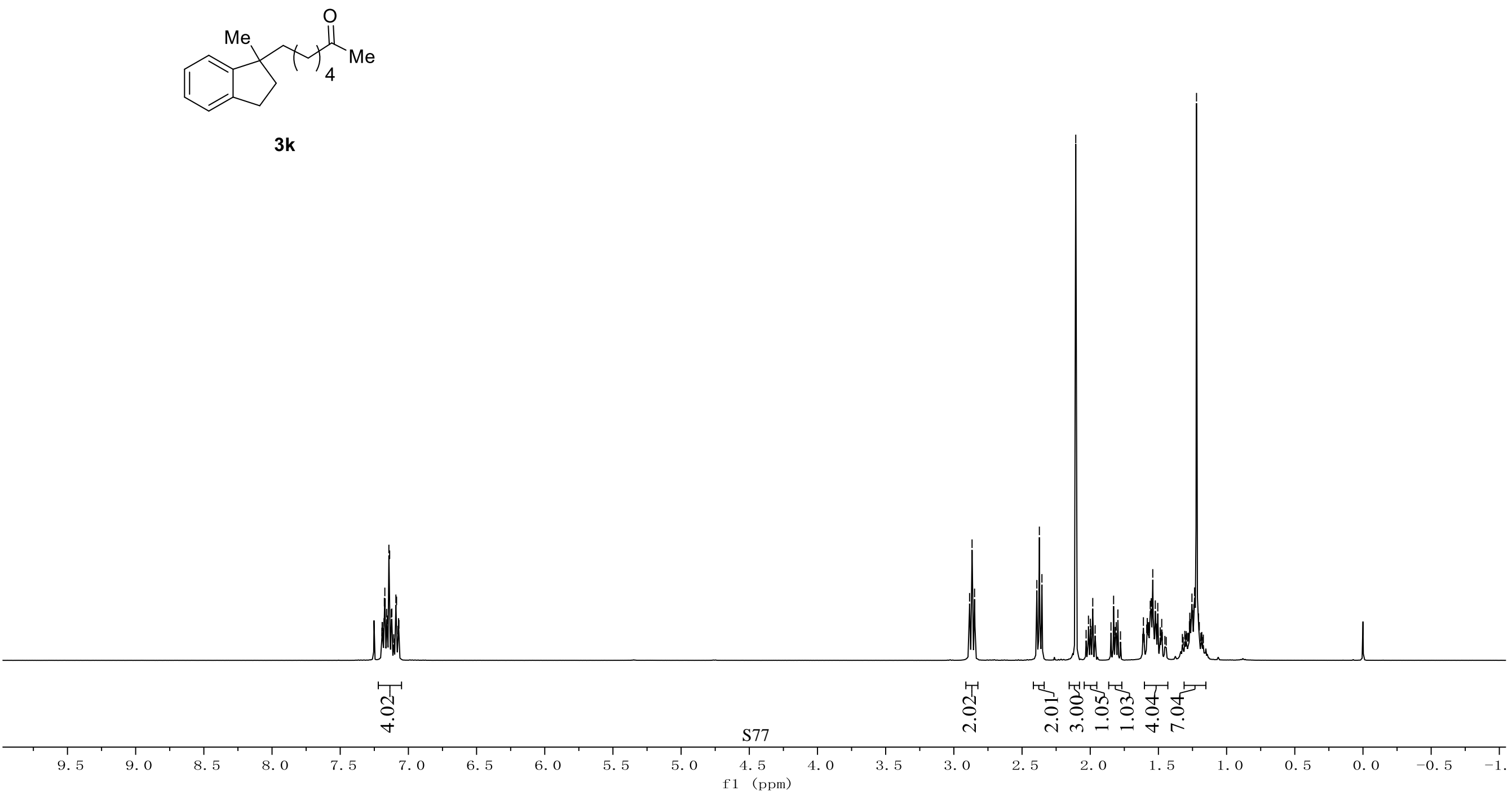


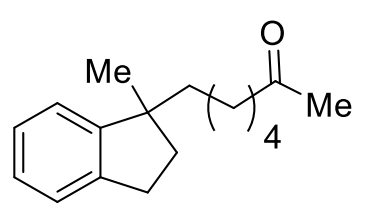

3k

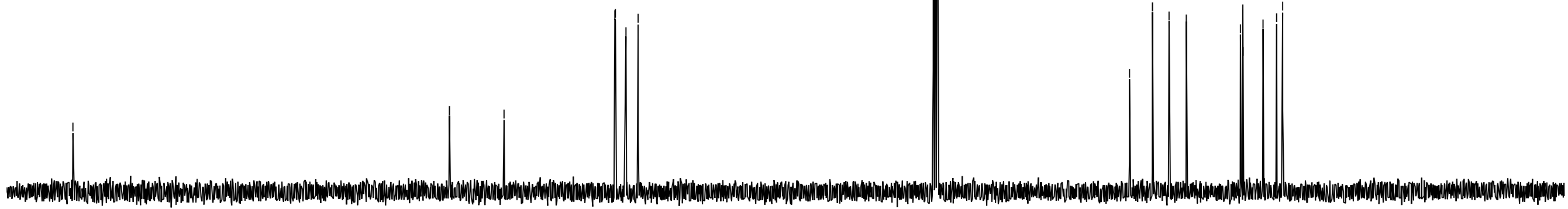

S78

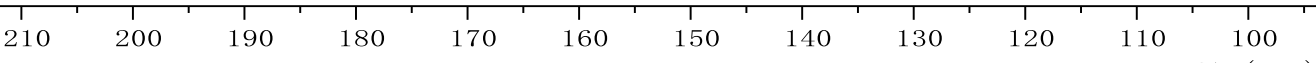

1 (ppm)
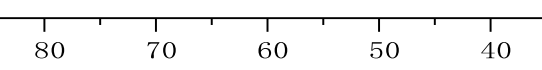


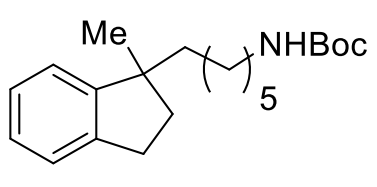

$3 \mathbf{I}$

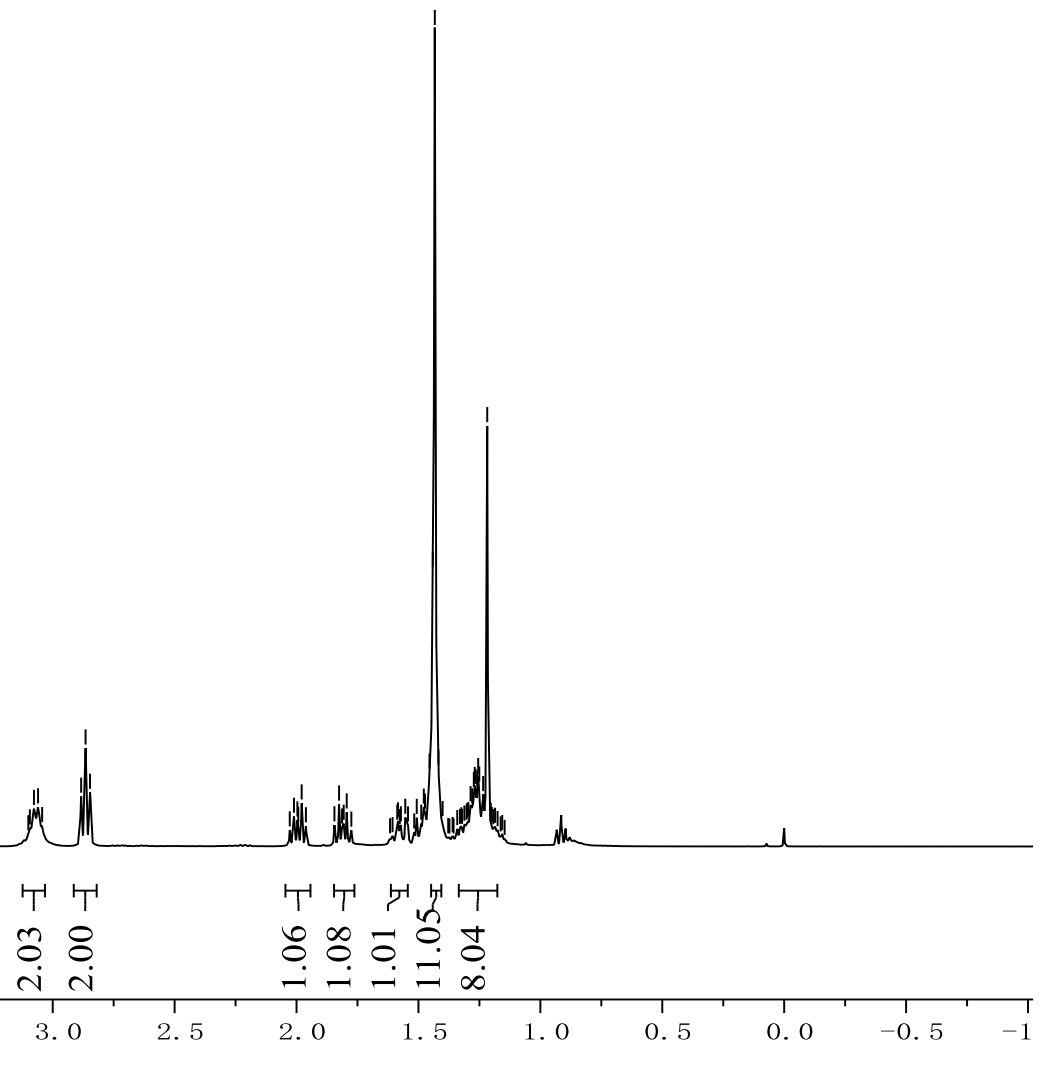




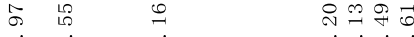

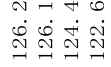

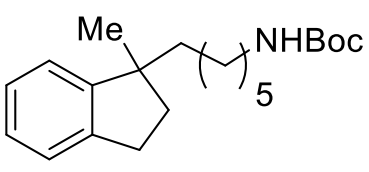

31
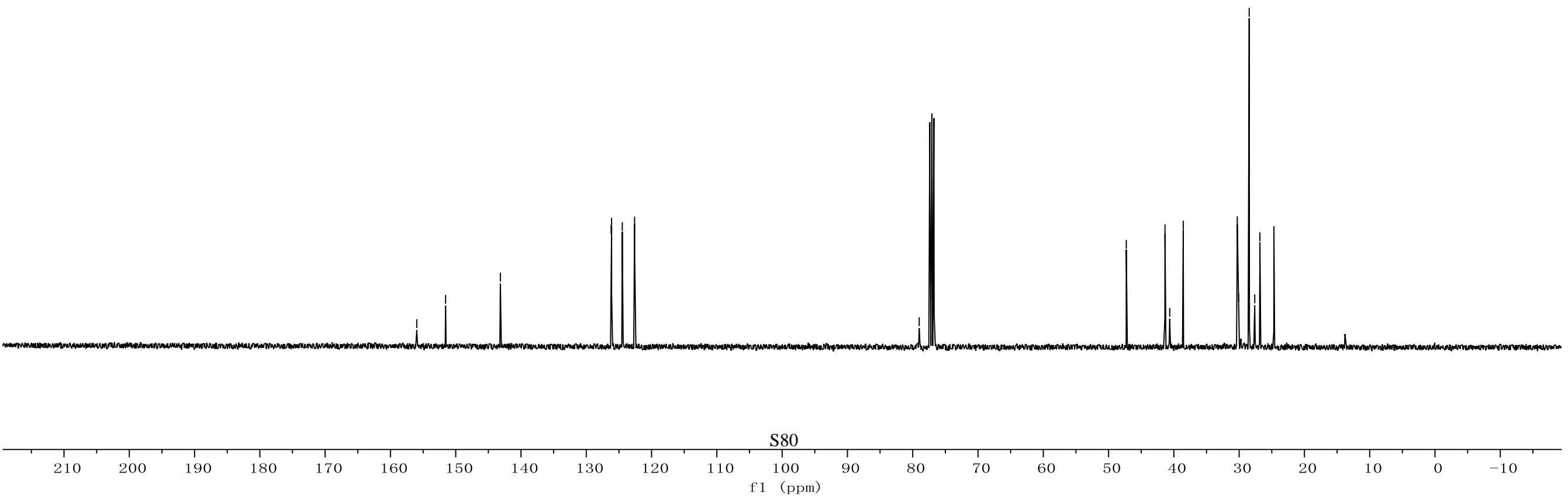

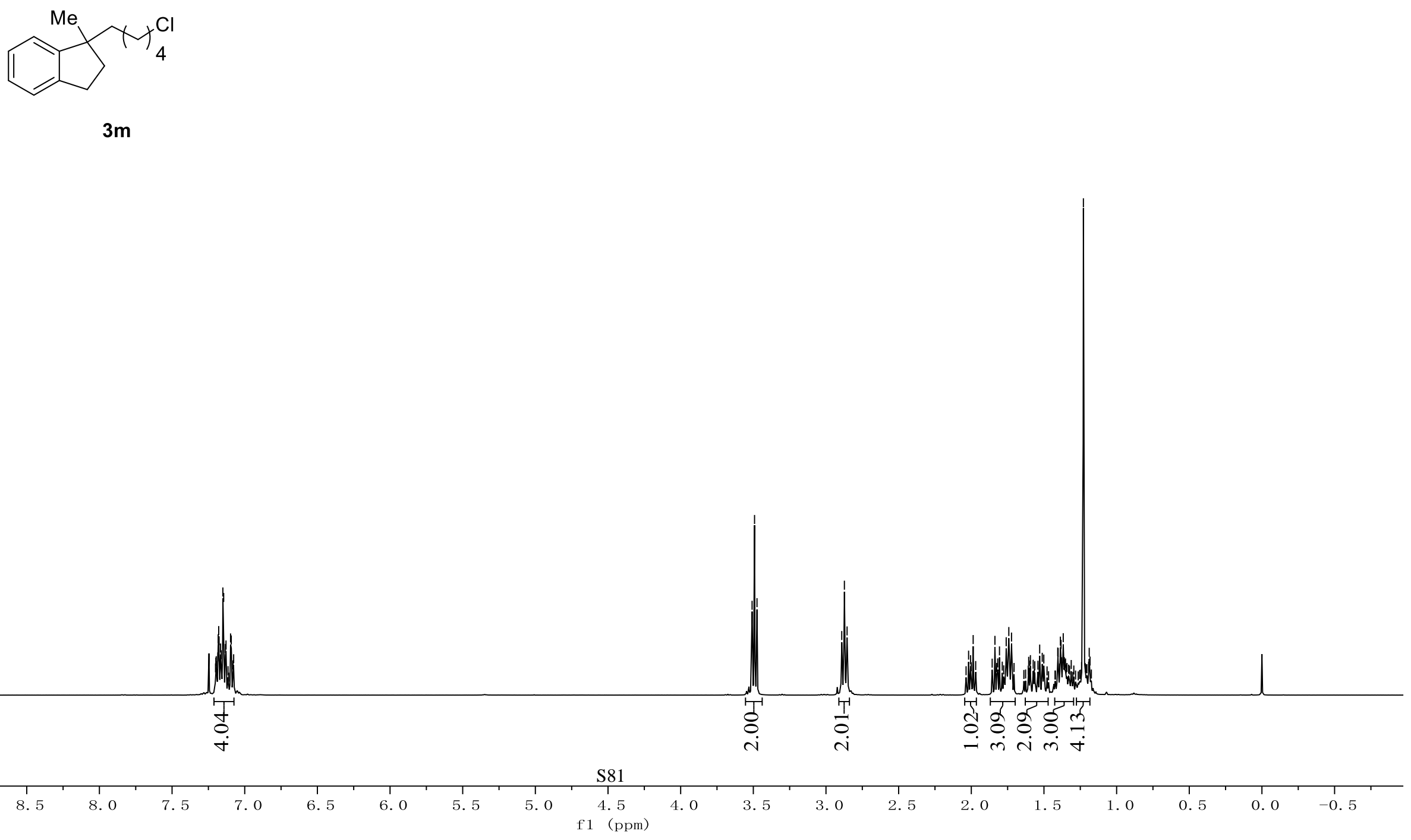


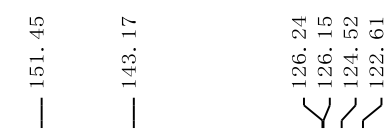

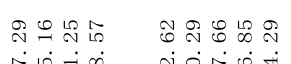

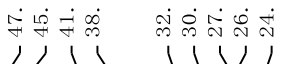

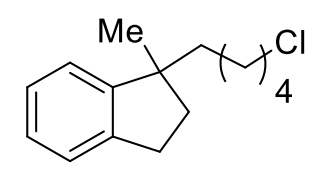

$3 m$

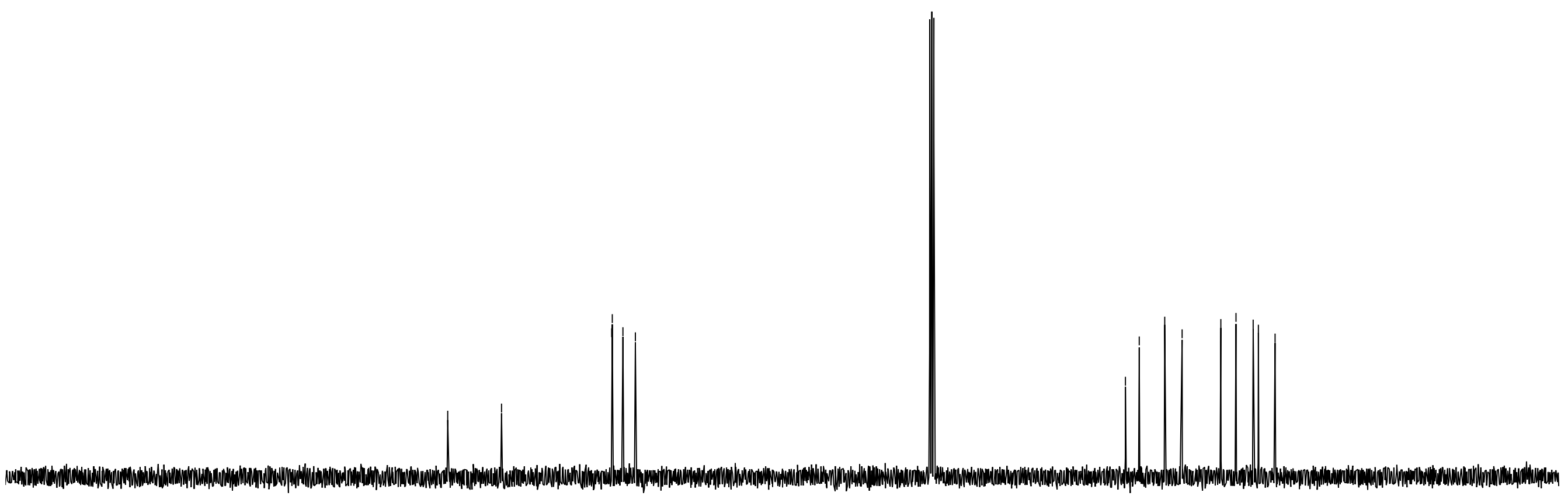




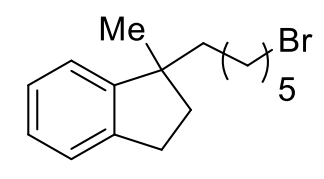

$3 n$

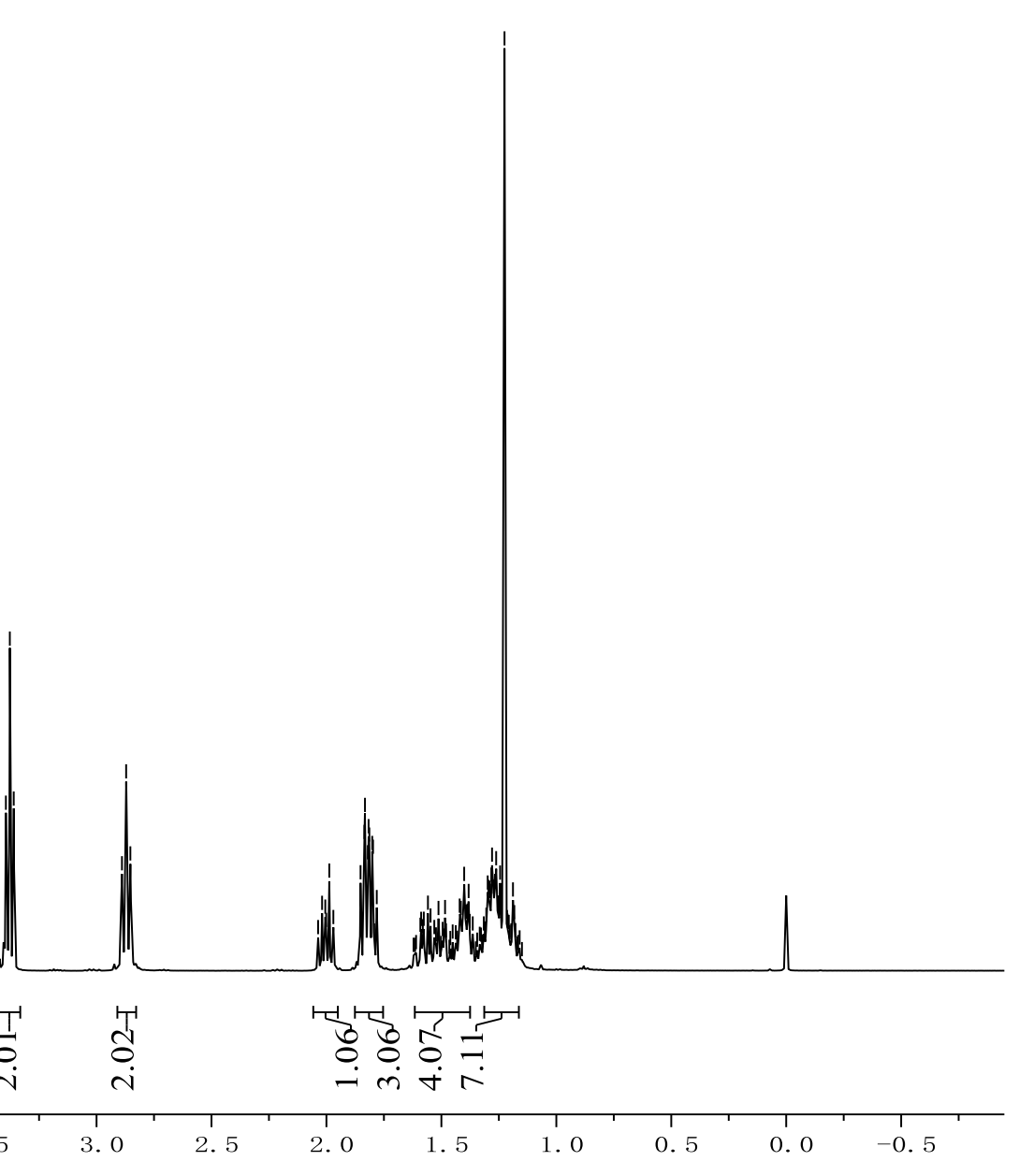




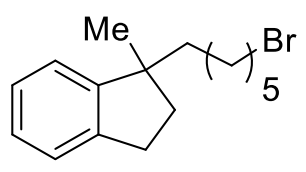

$3 n$

$110>111$

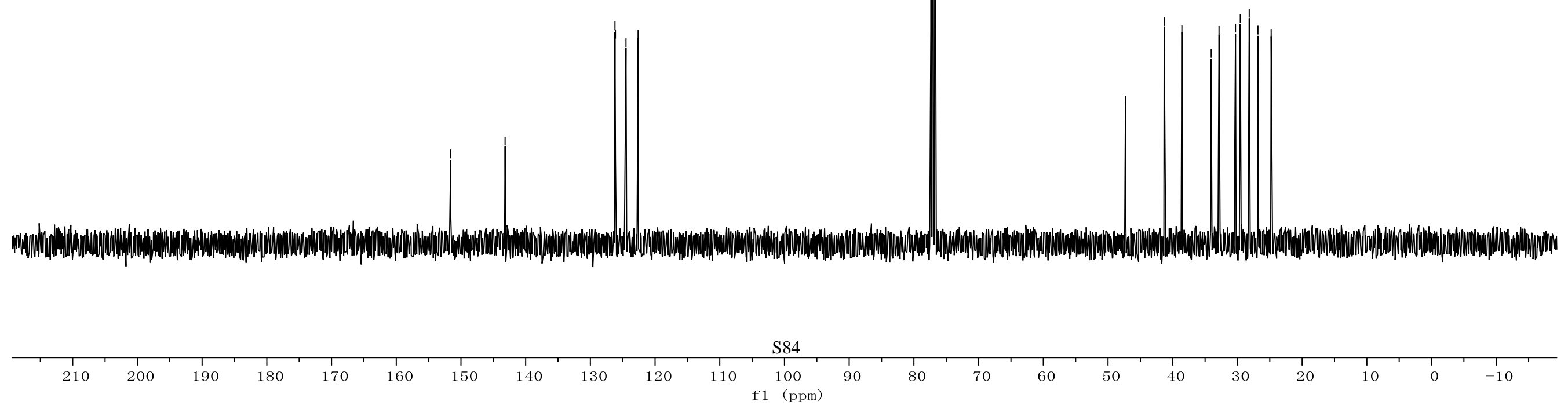




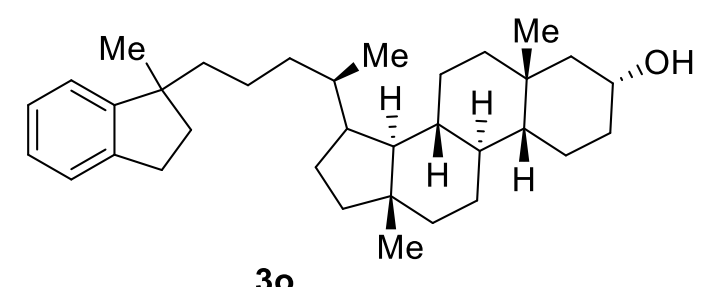

30

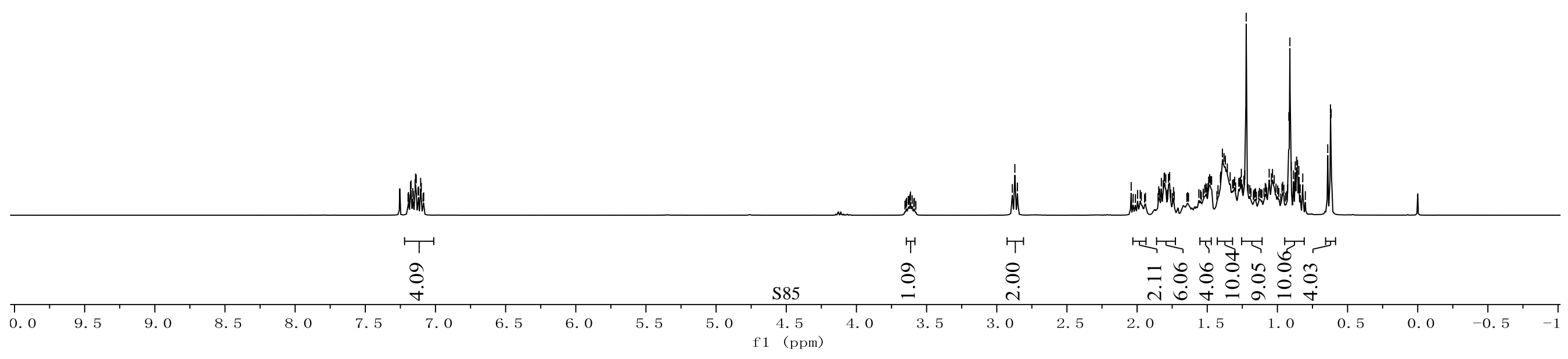




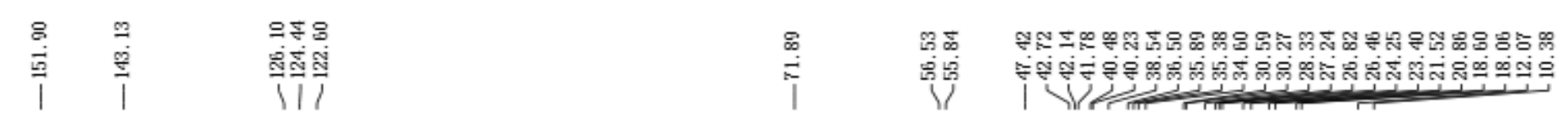

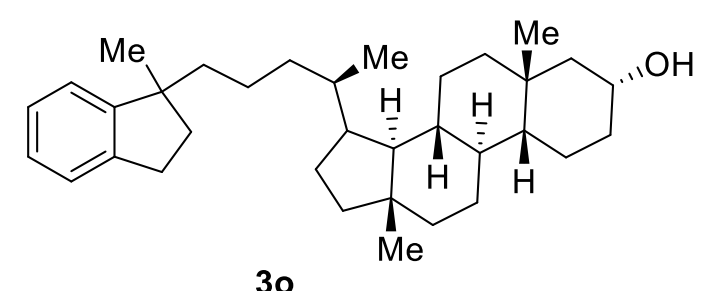

30

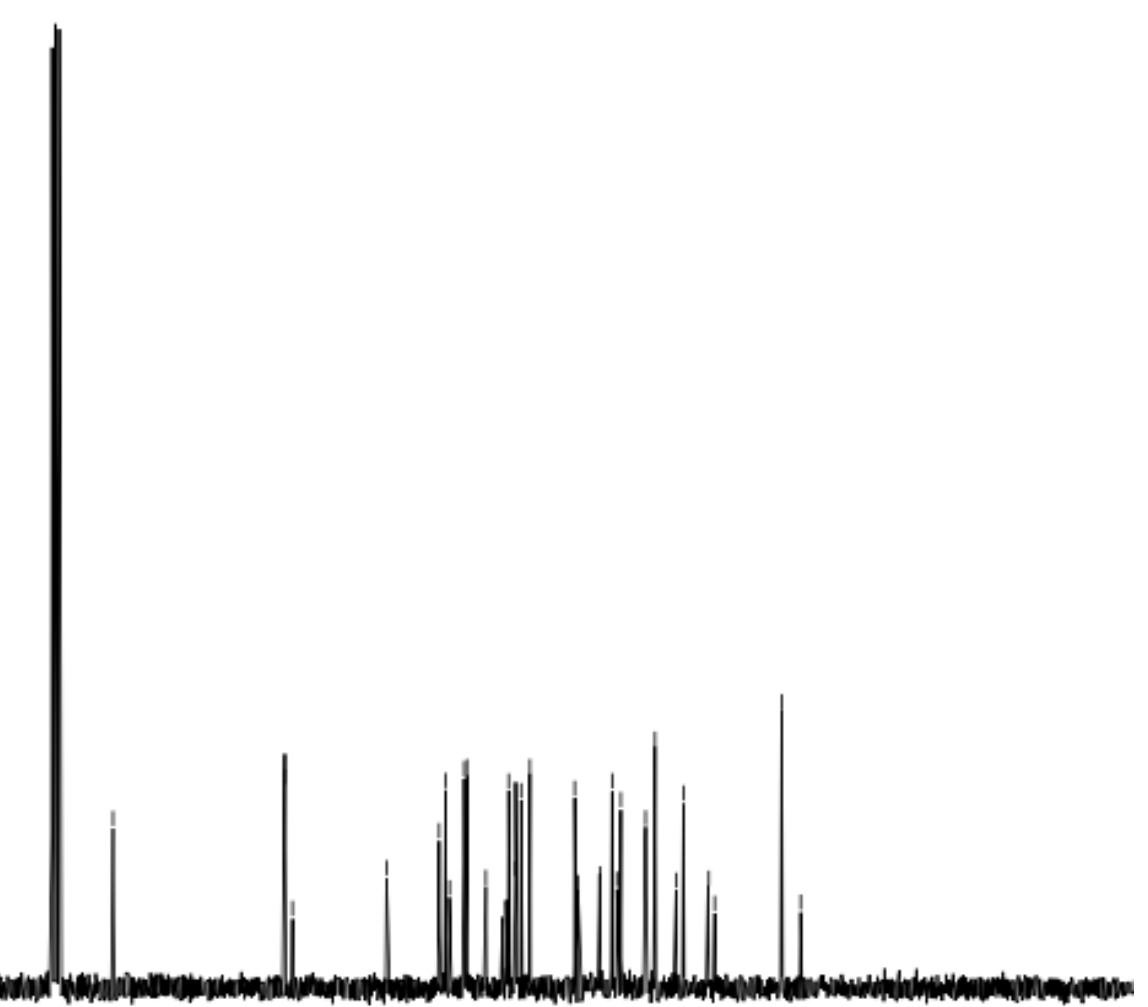




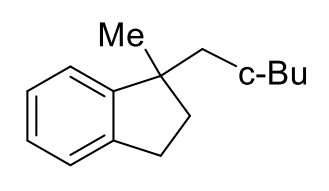

$3 p$

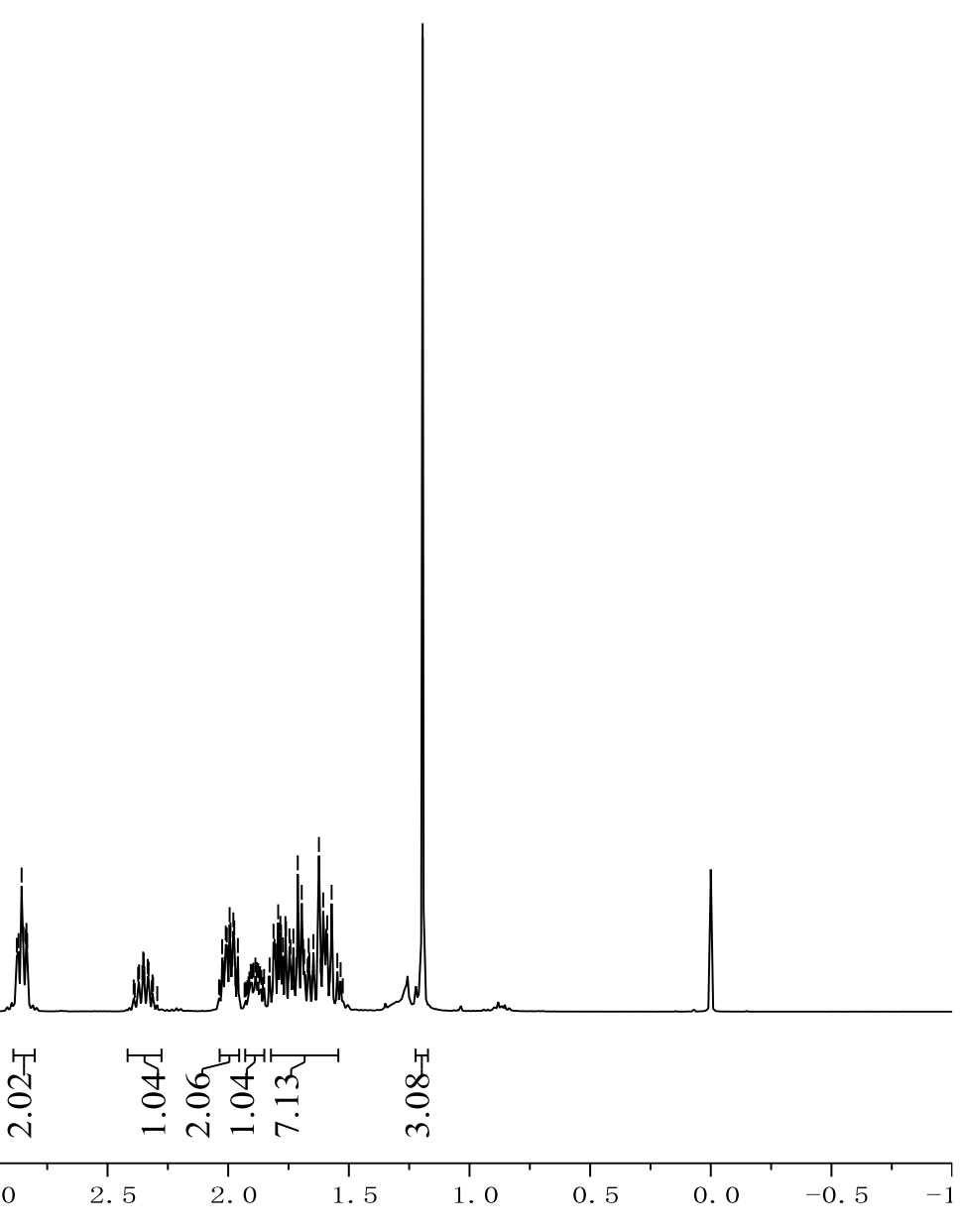




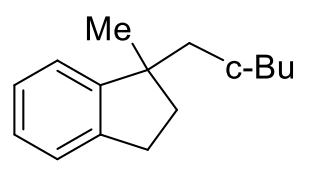

$3 p$

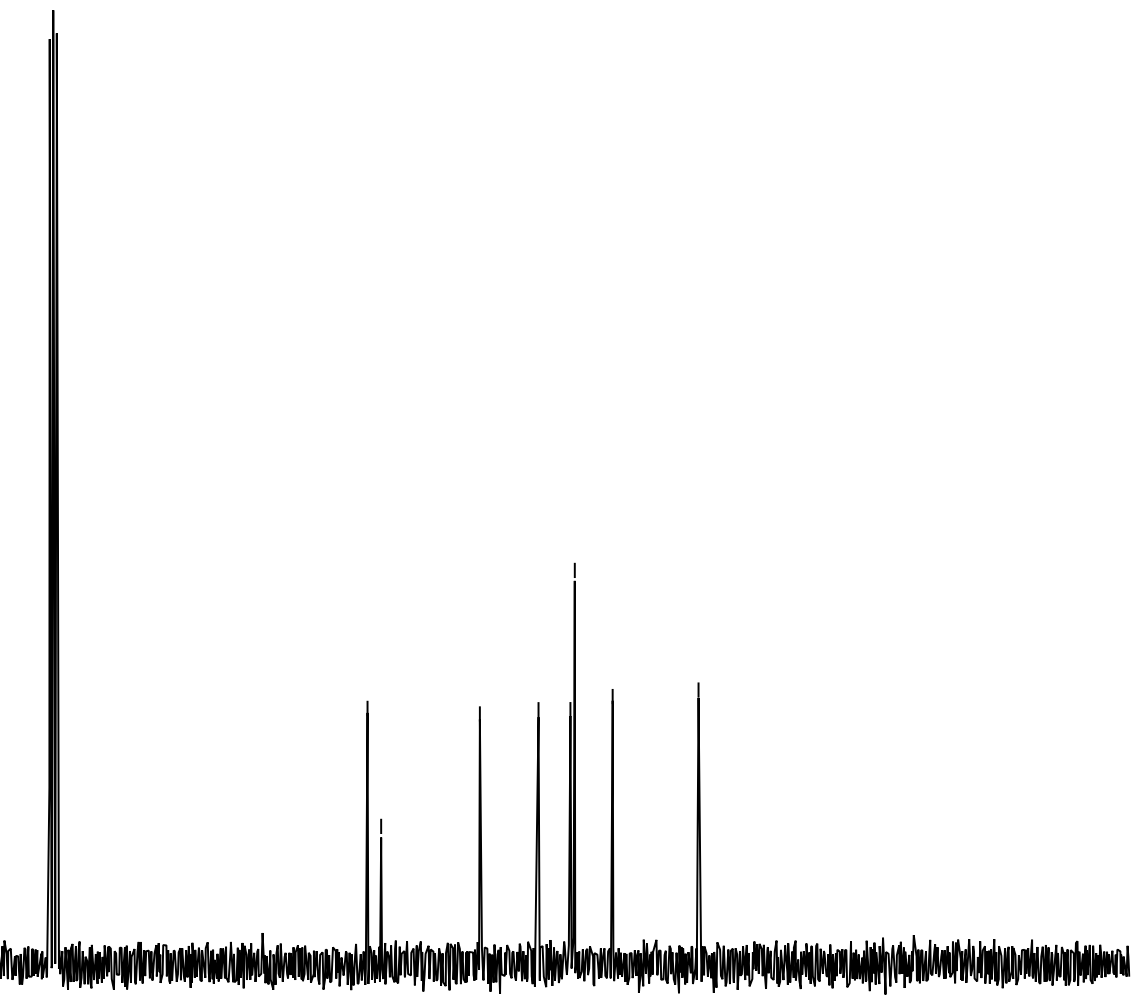




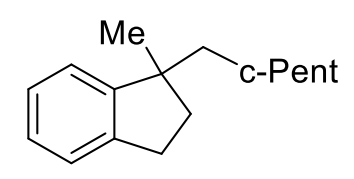

$3 q$

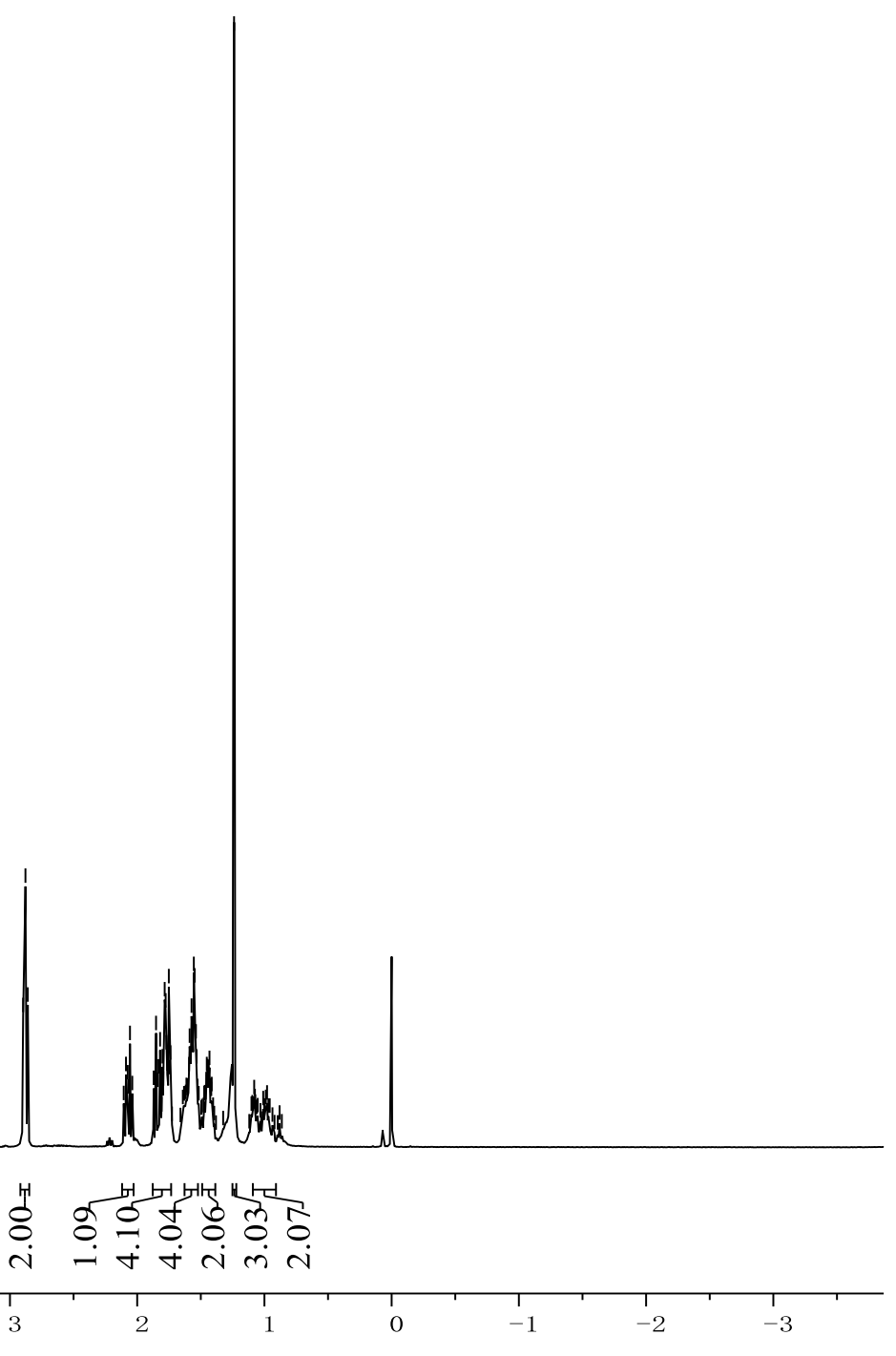




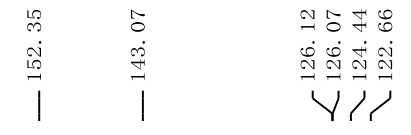

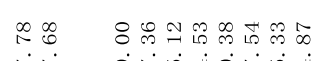
Y

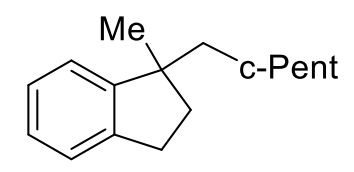

$3 q$

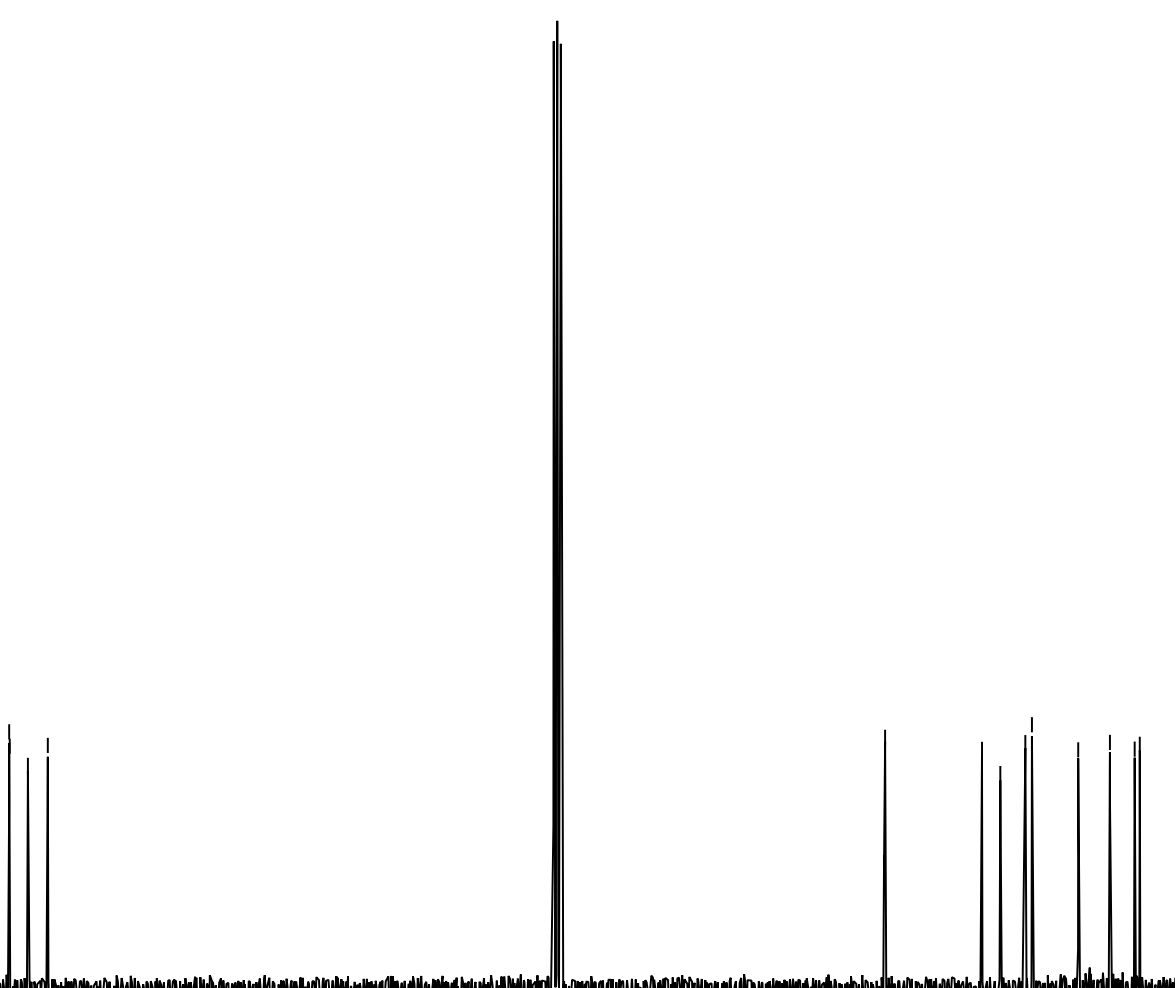

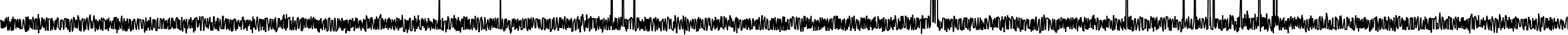

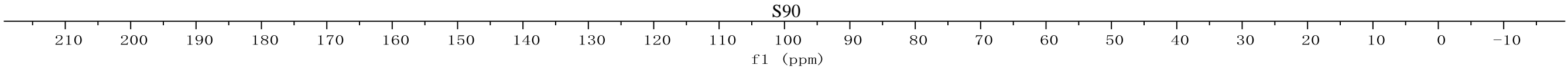



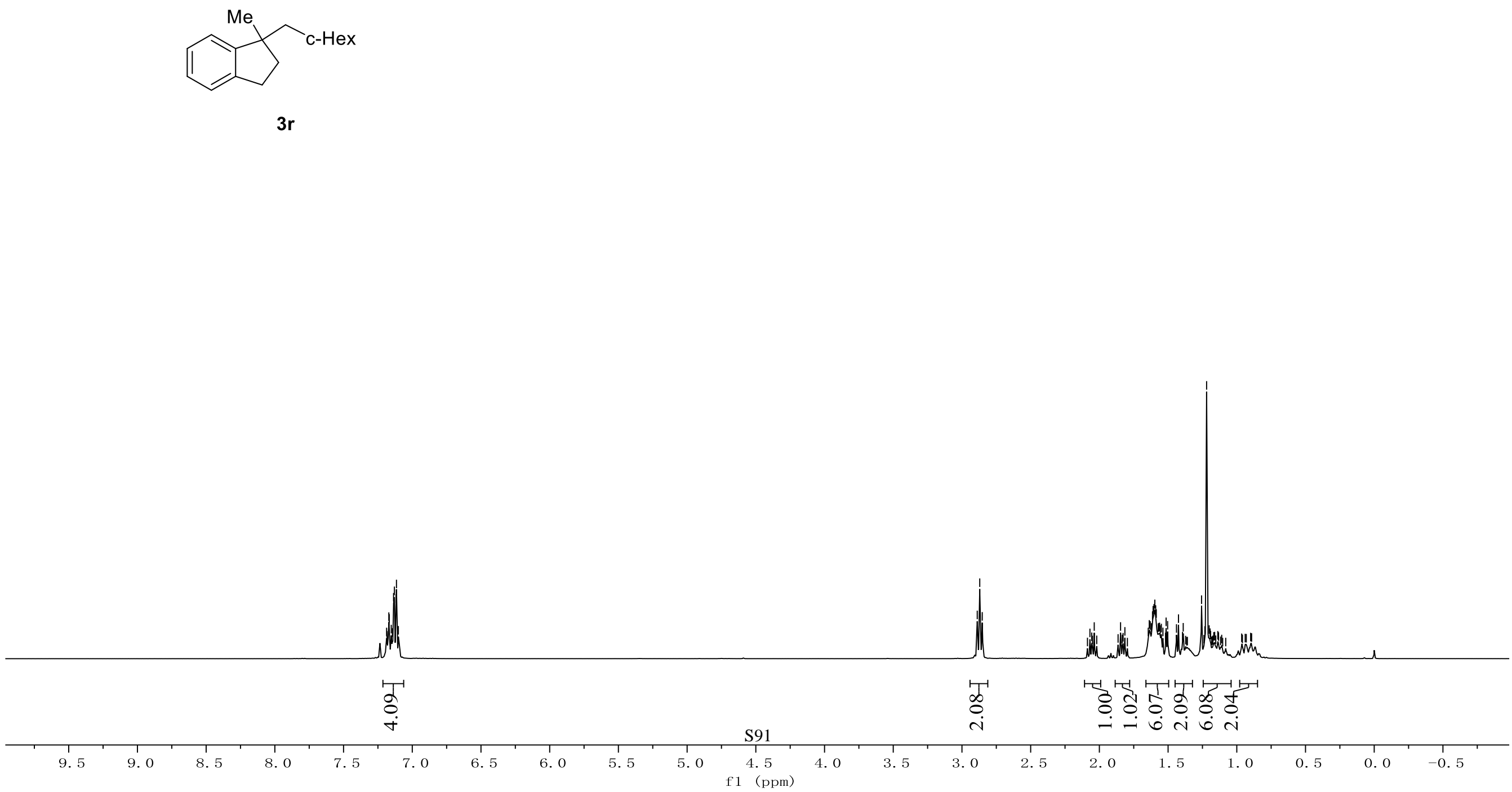

की 8 의

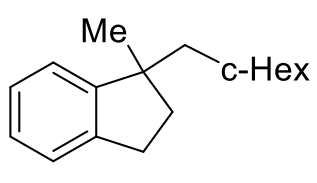

$3 r$
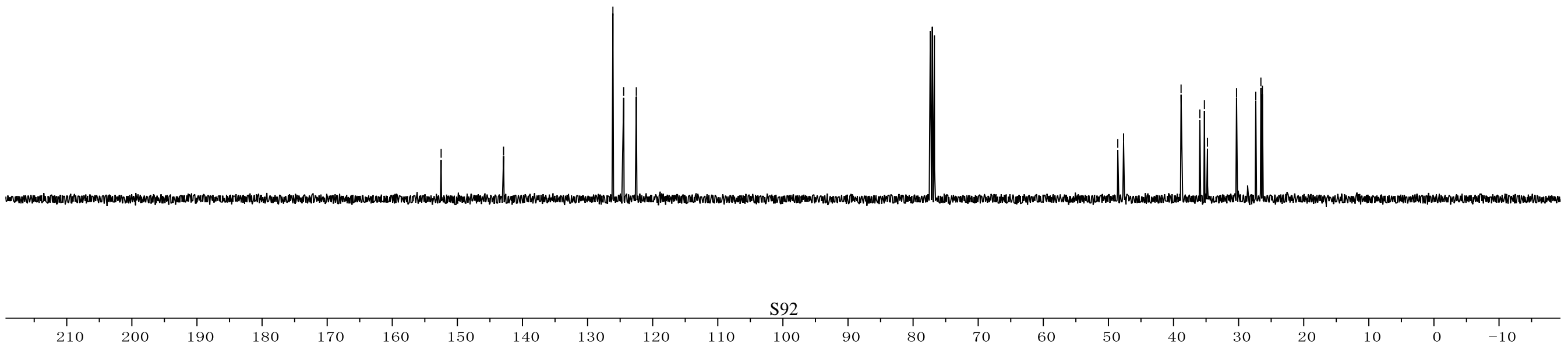


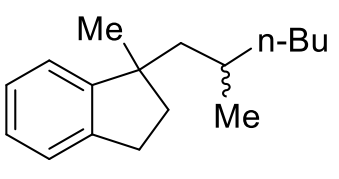

$3 s$

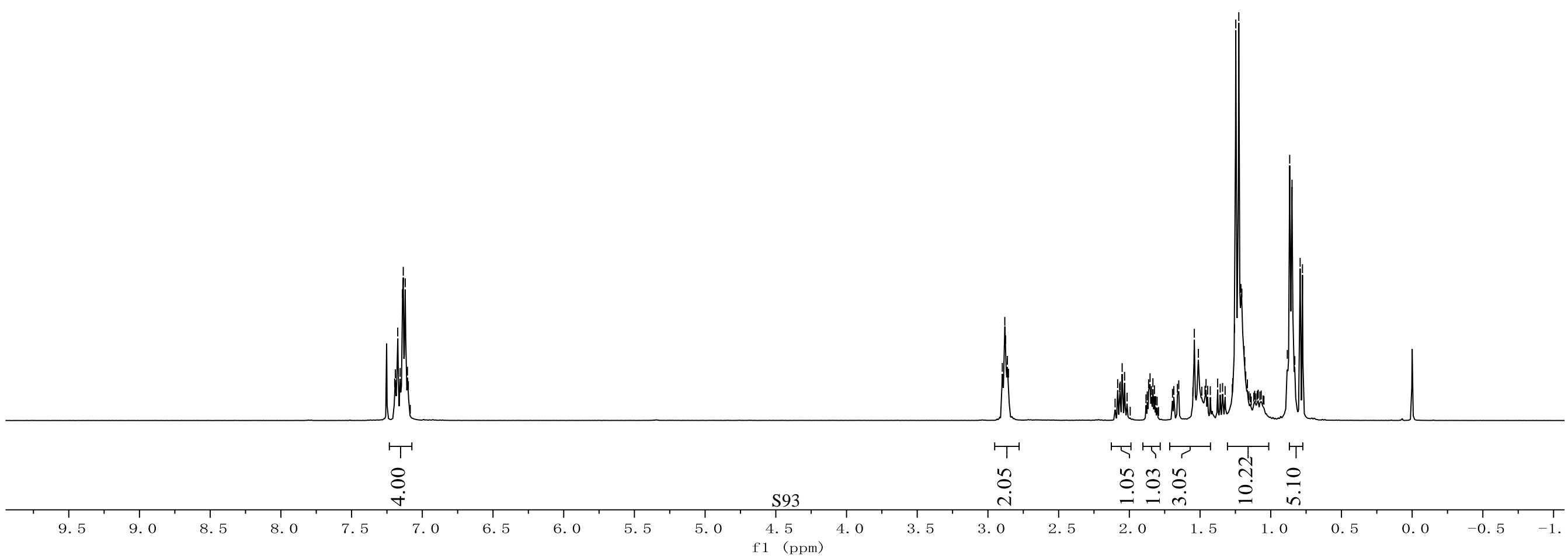




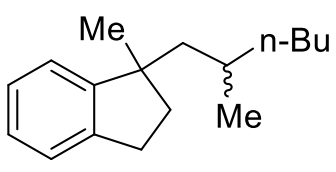

$3 s$

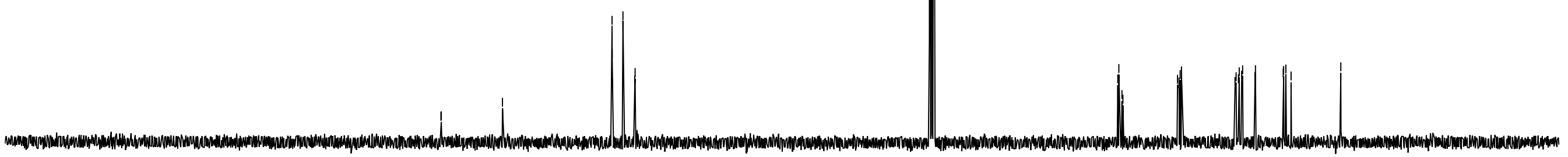

\begin{tabular}{|c|c|c|c|c|c|c|c|c|c|c|c|c|c|c|c|c|c|c|c|c|c|c|}
\hline & & & & & & & & & & & S94 & & & & & & & & & & & \\
\hline 210 & 200 & 190 & 180 & 170 & 160 & 150 & 140 & 130 & 120 & 110 & $\begin{array}{l}100 \\
1 \quad(\mathrm{ppm})\end{array}$ & 90 & 80 & 70 & 60 & 50 & 40 & 30 & 20 & 10 & 0 & -10 \\
\hline
\end{tabular}




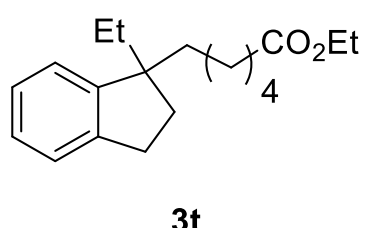

$3 t$

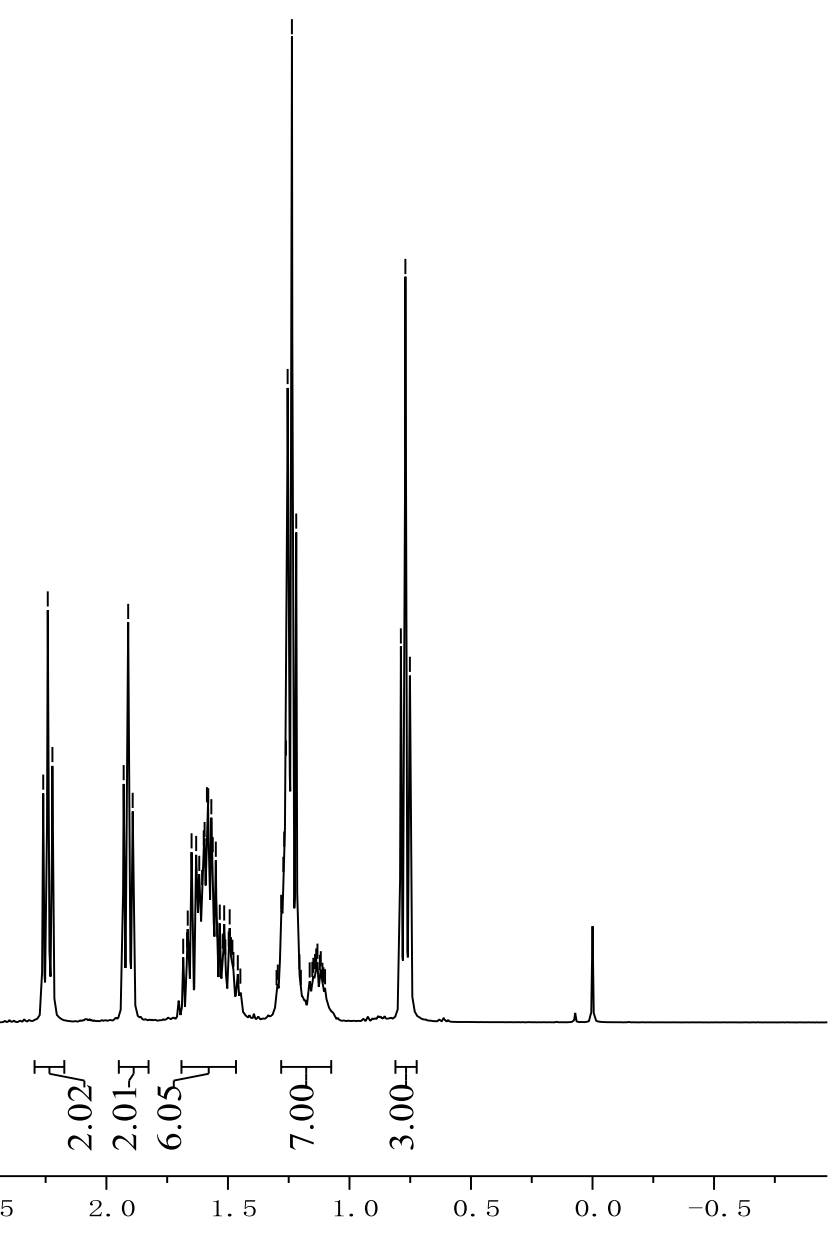




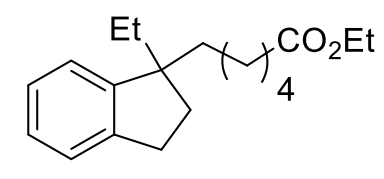

$3 t$
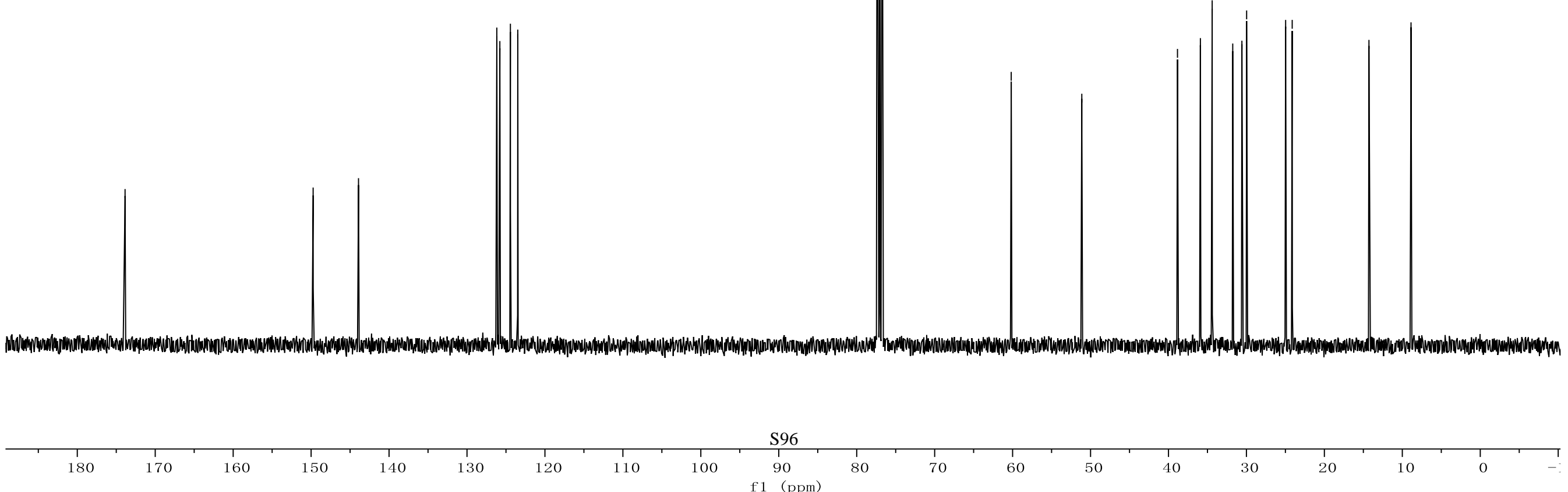


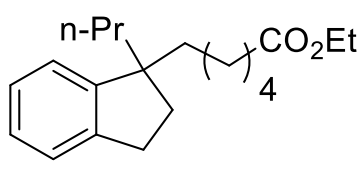

$3 u$

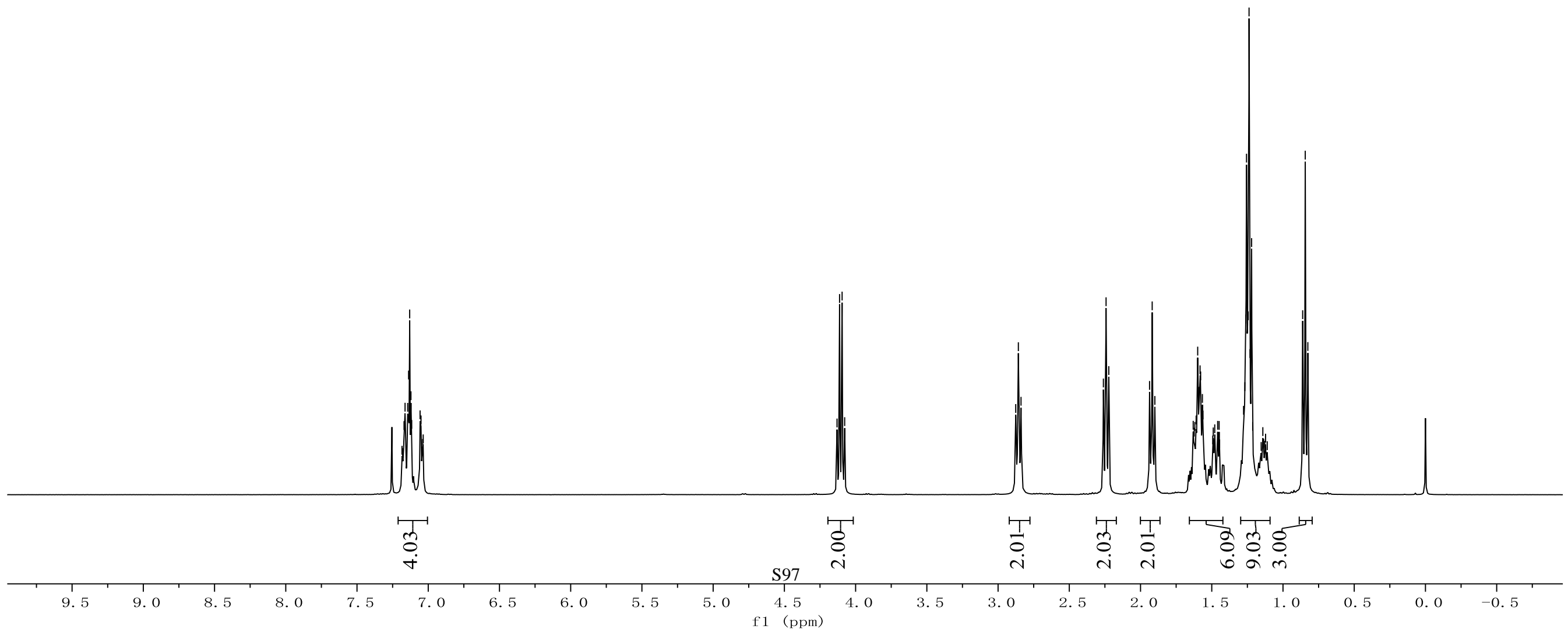



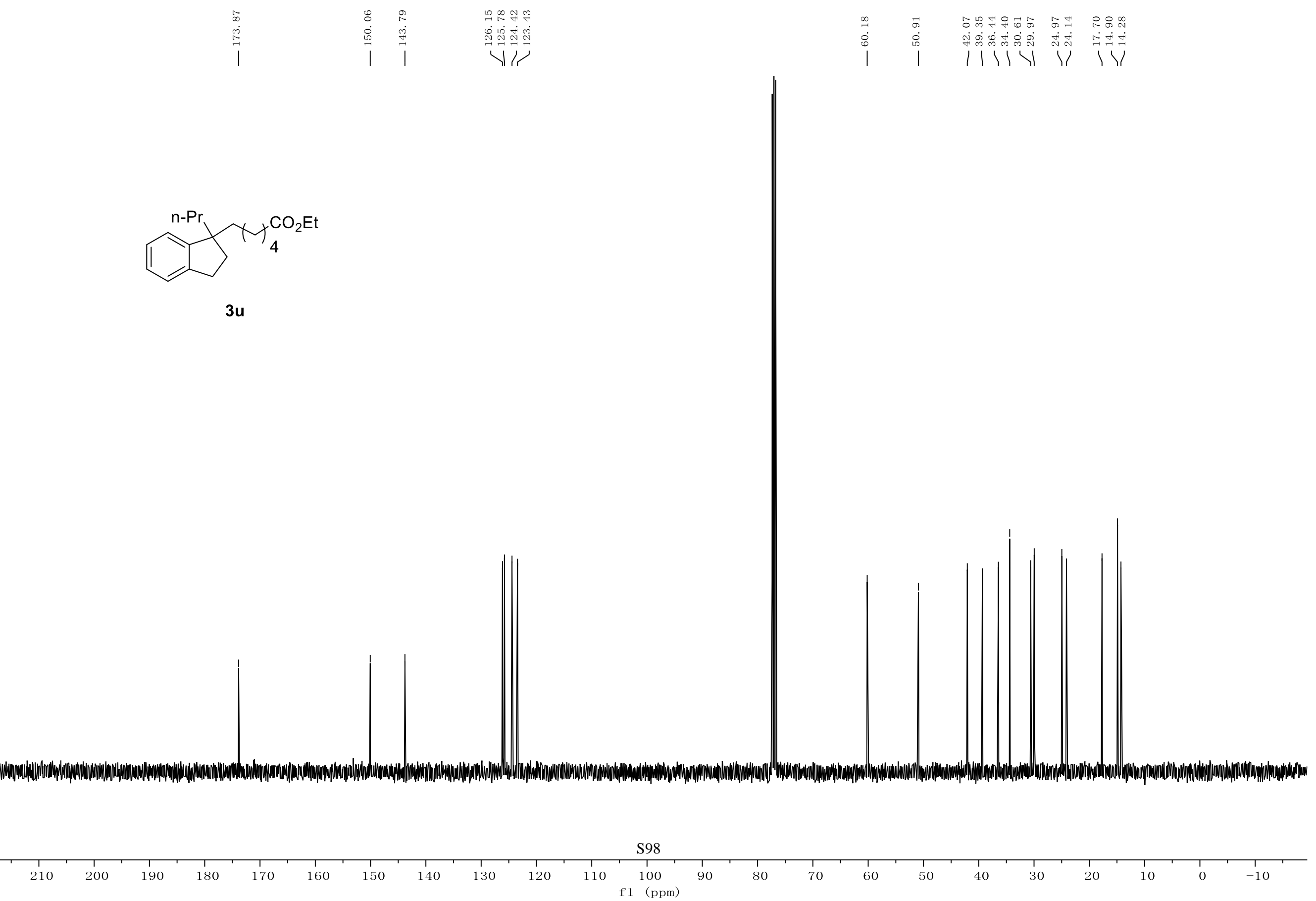


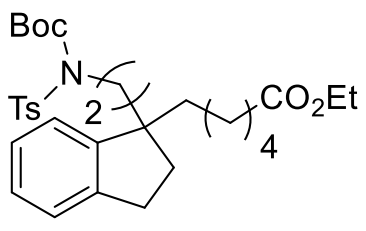

3v

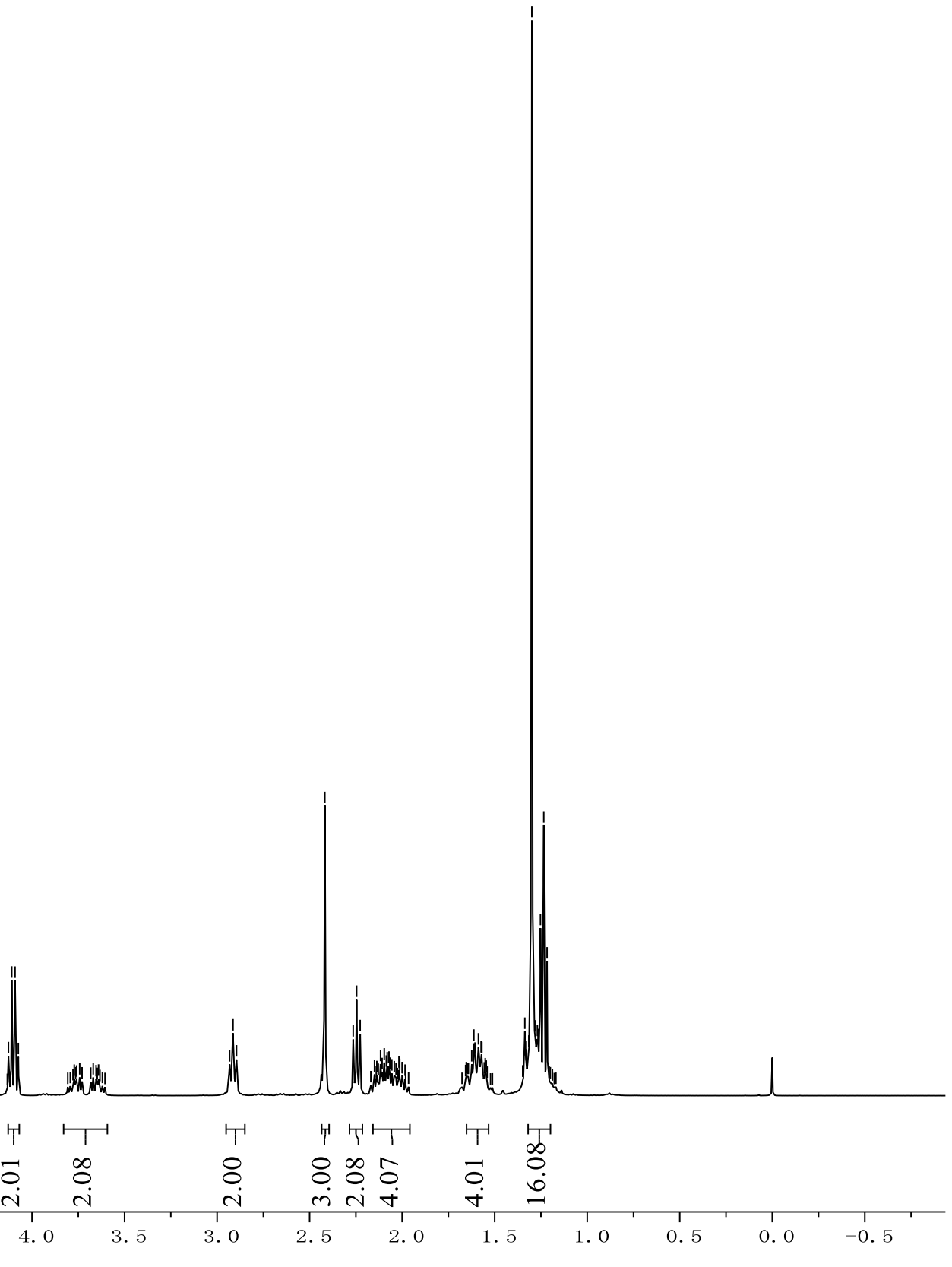




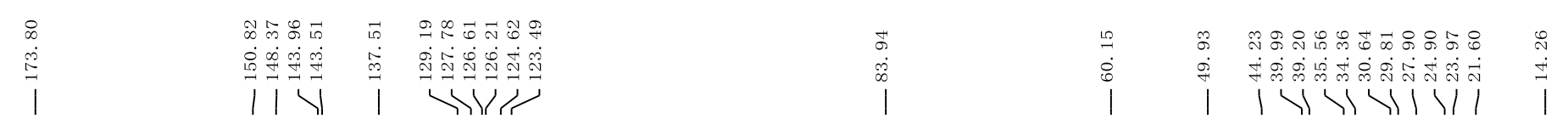

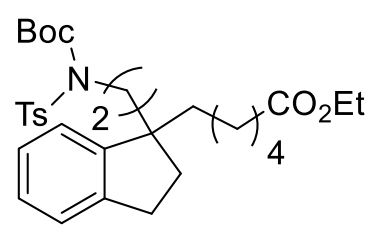

3v
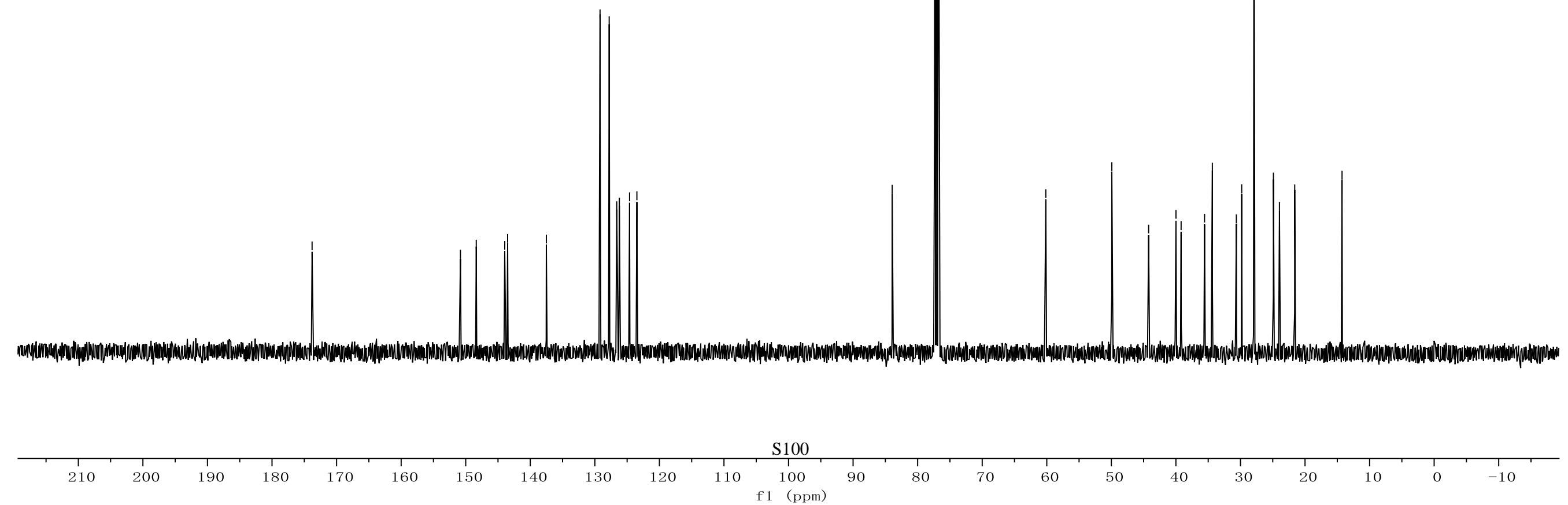

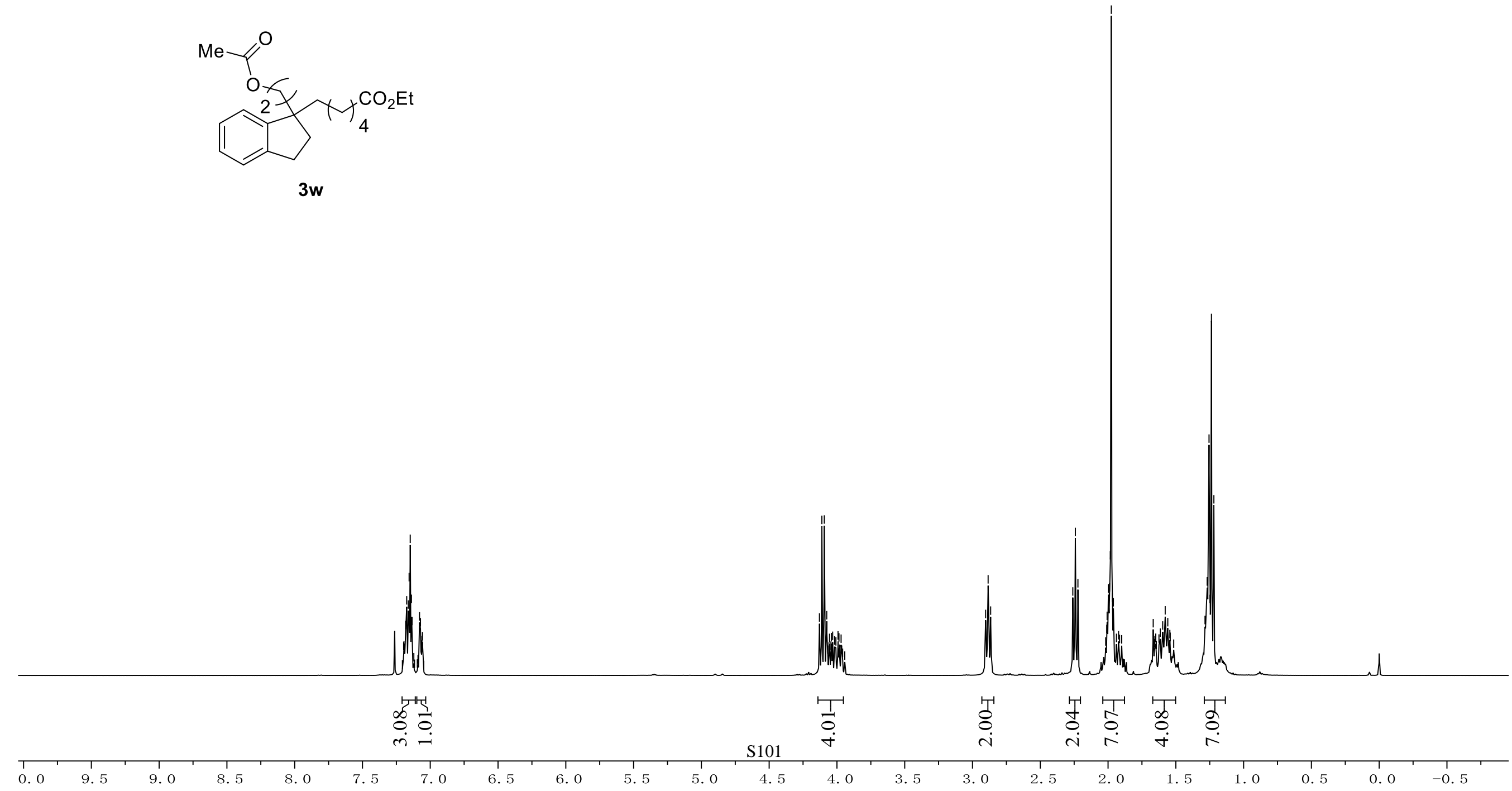


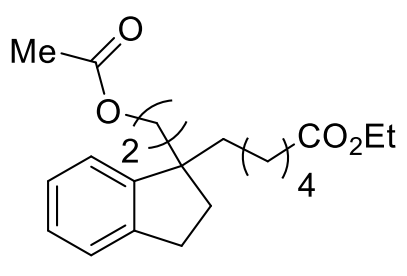

$3 \mathbf{w}$

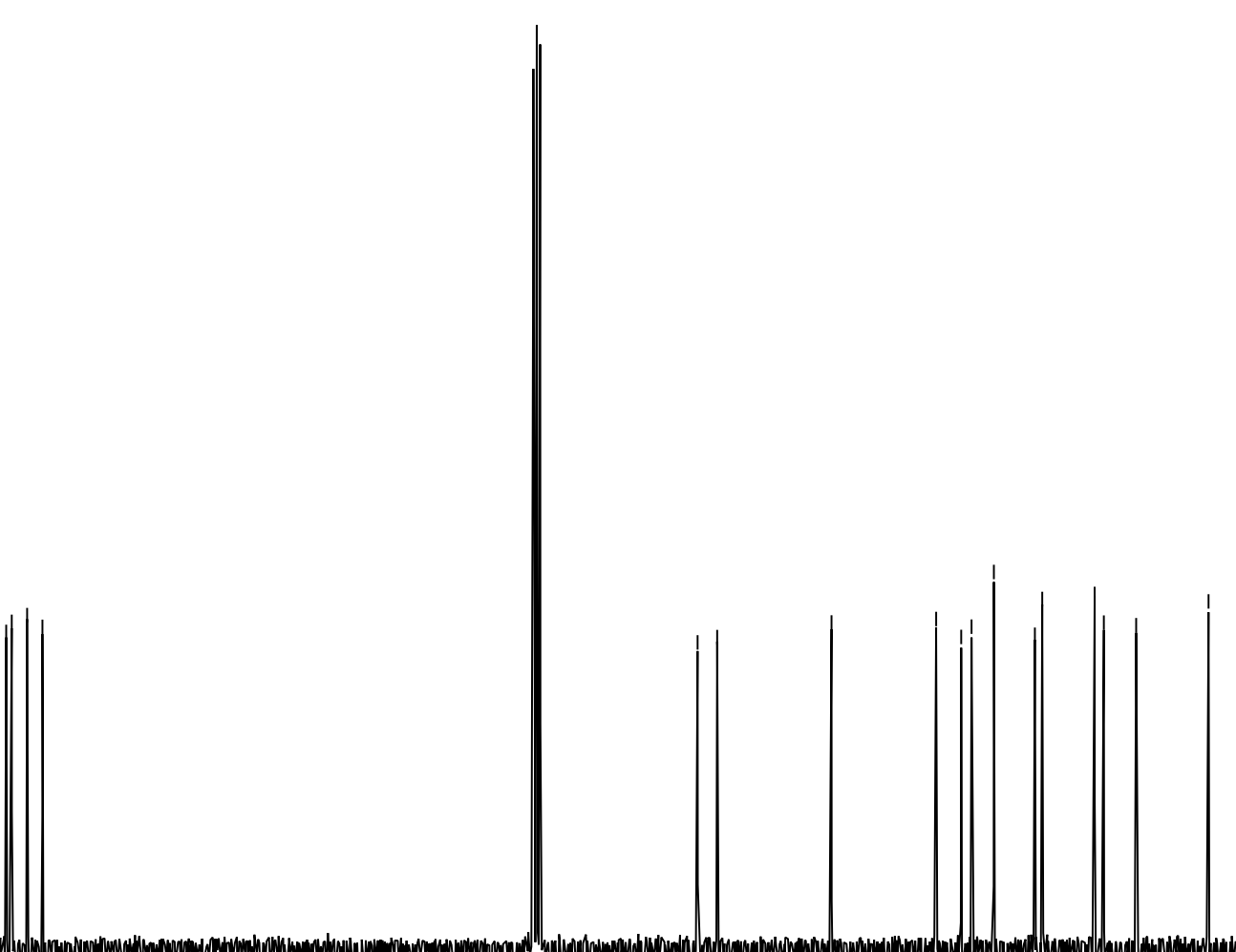

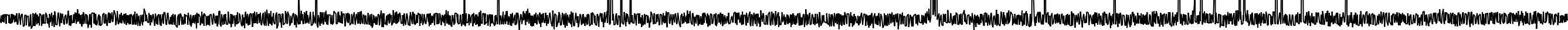

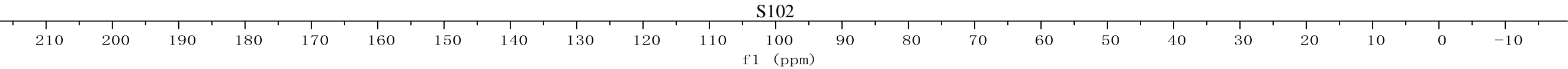



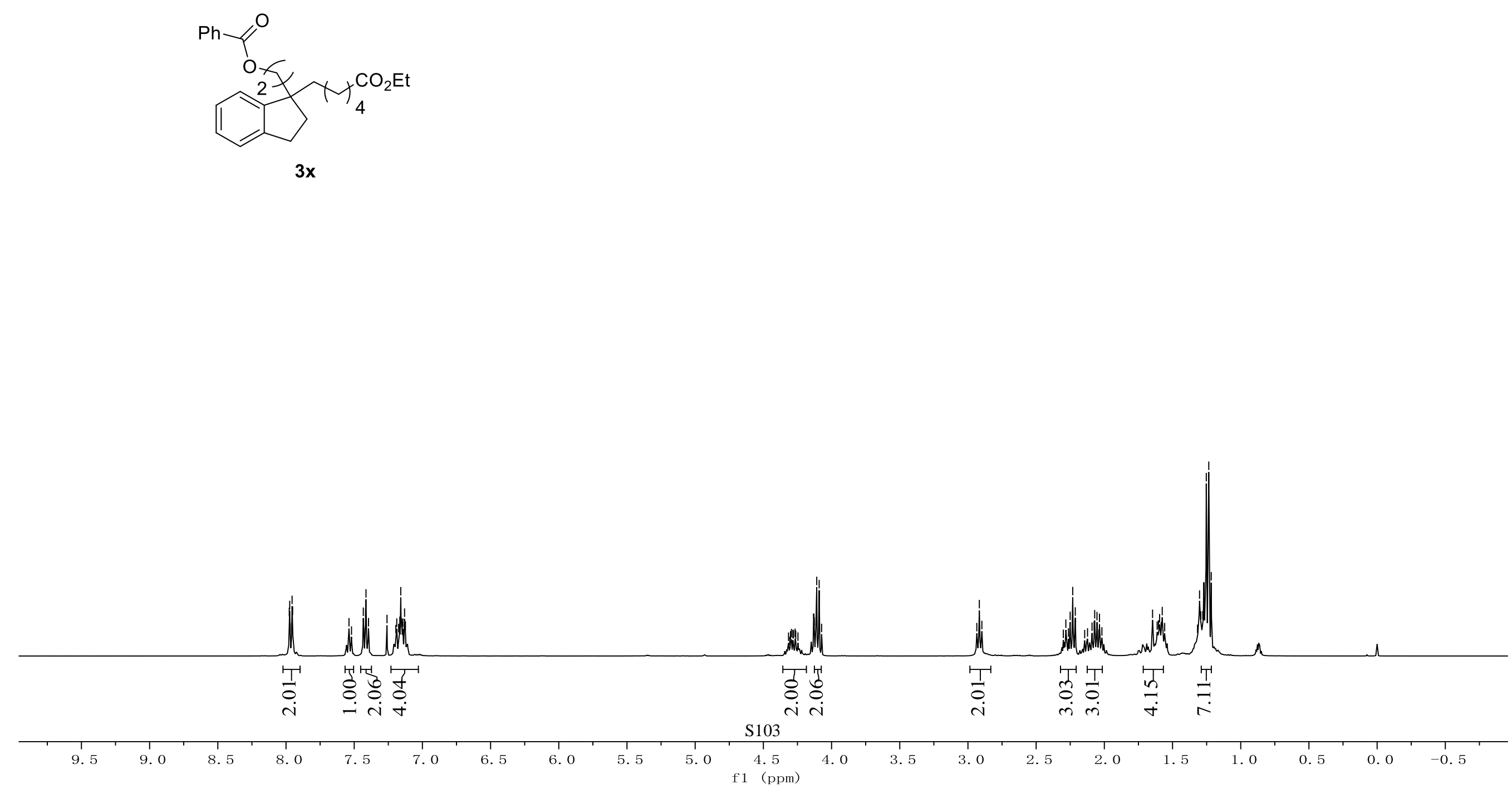


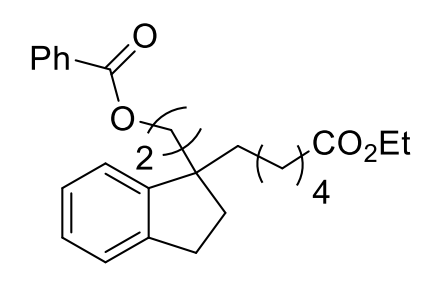

$3 x$
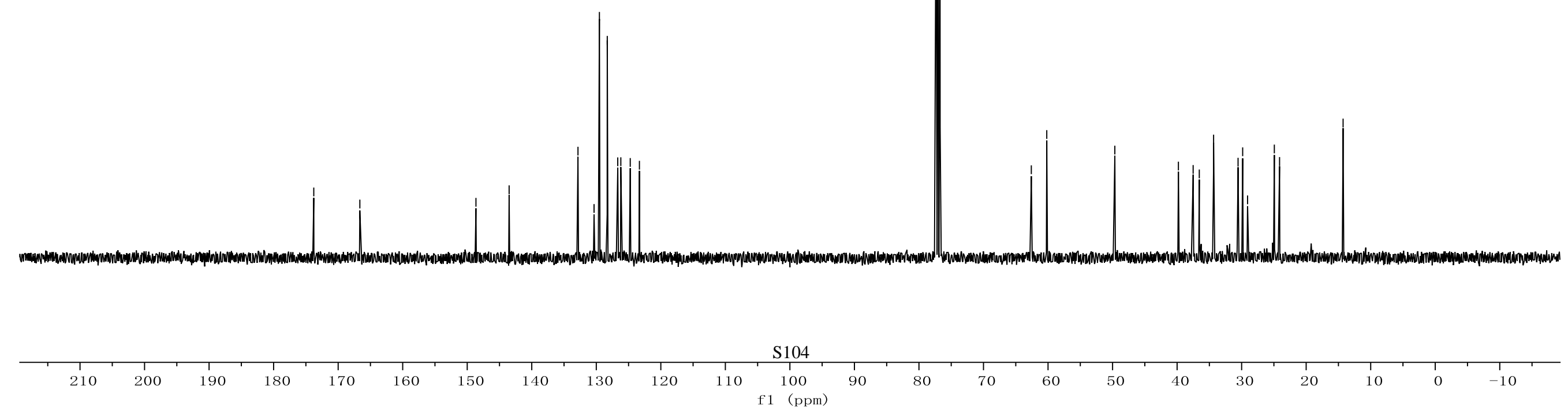

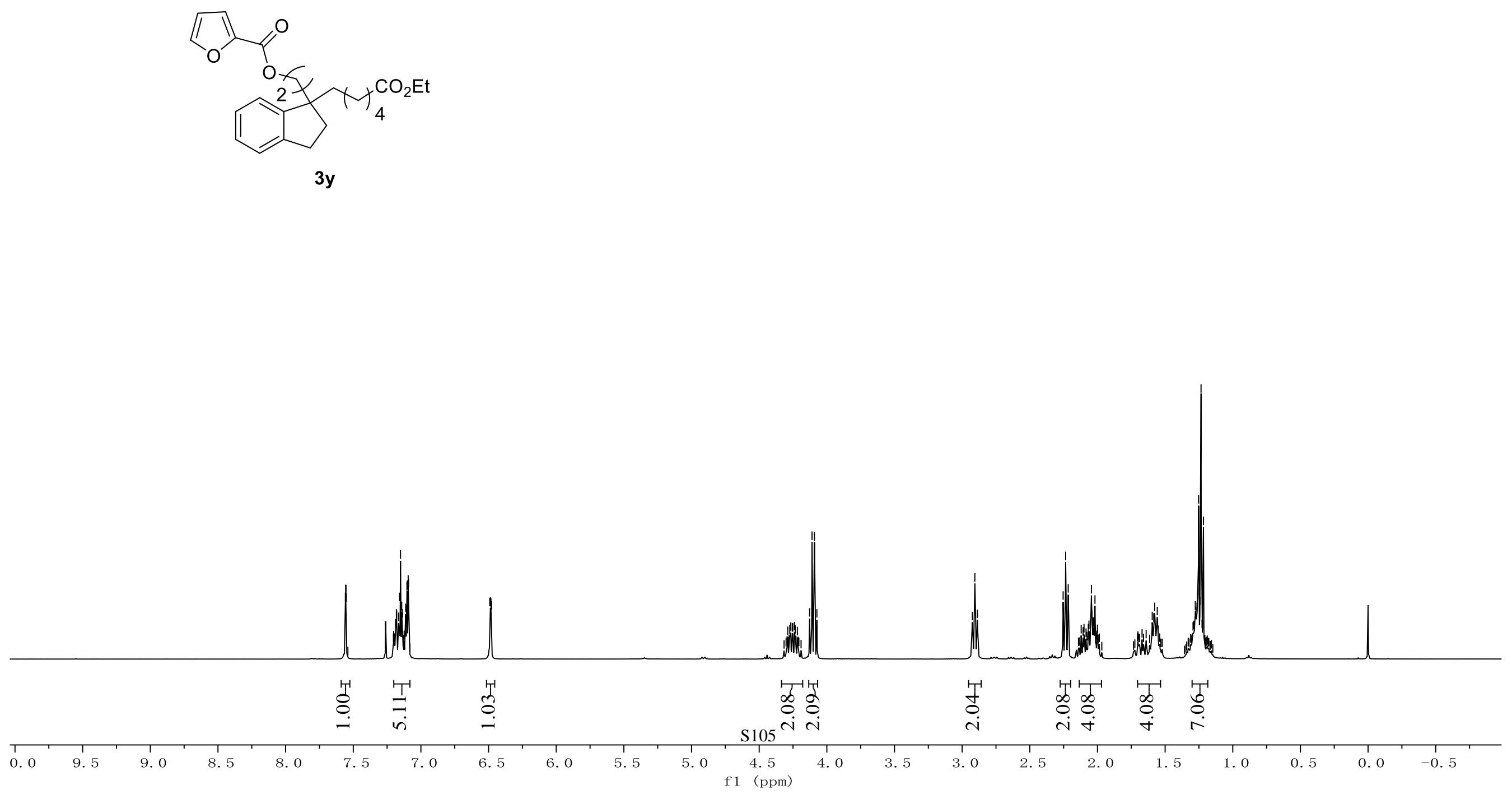


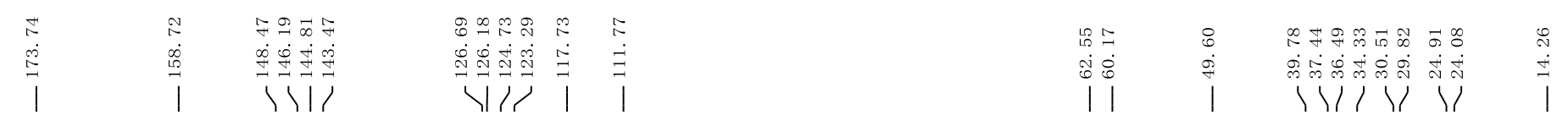

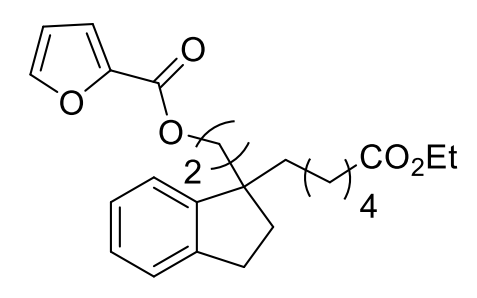

3y
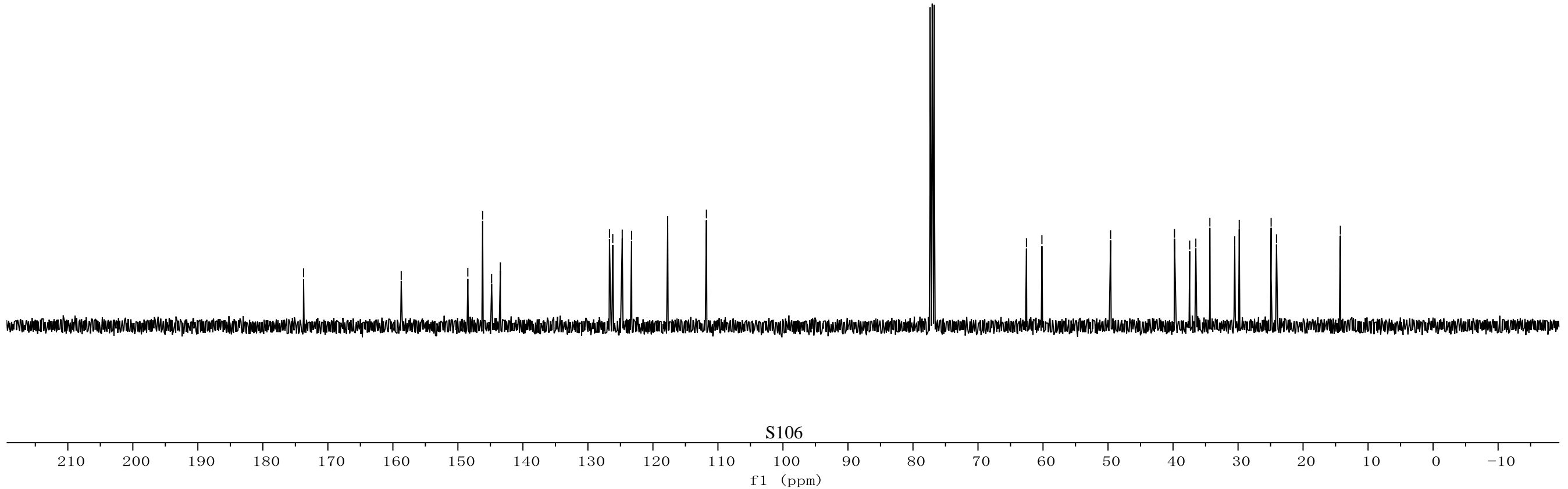

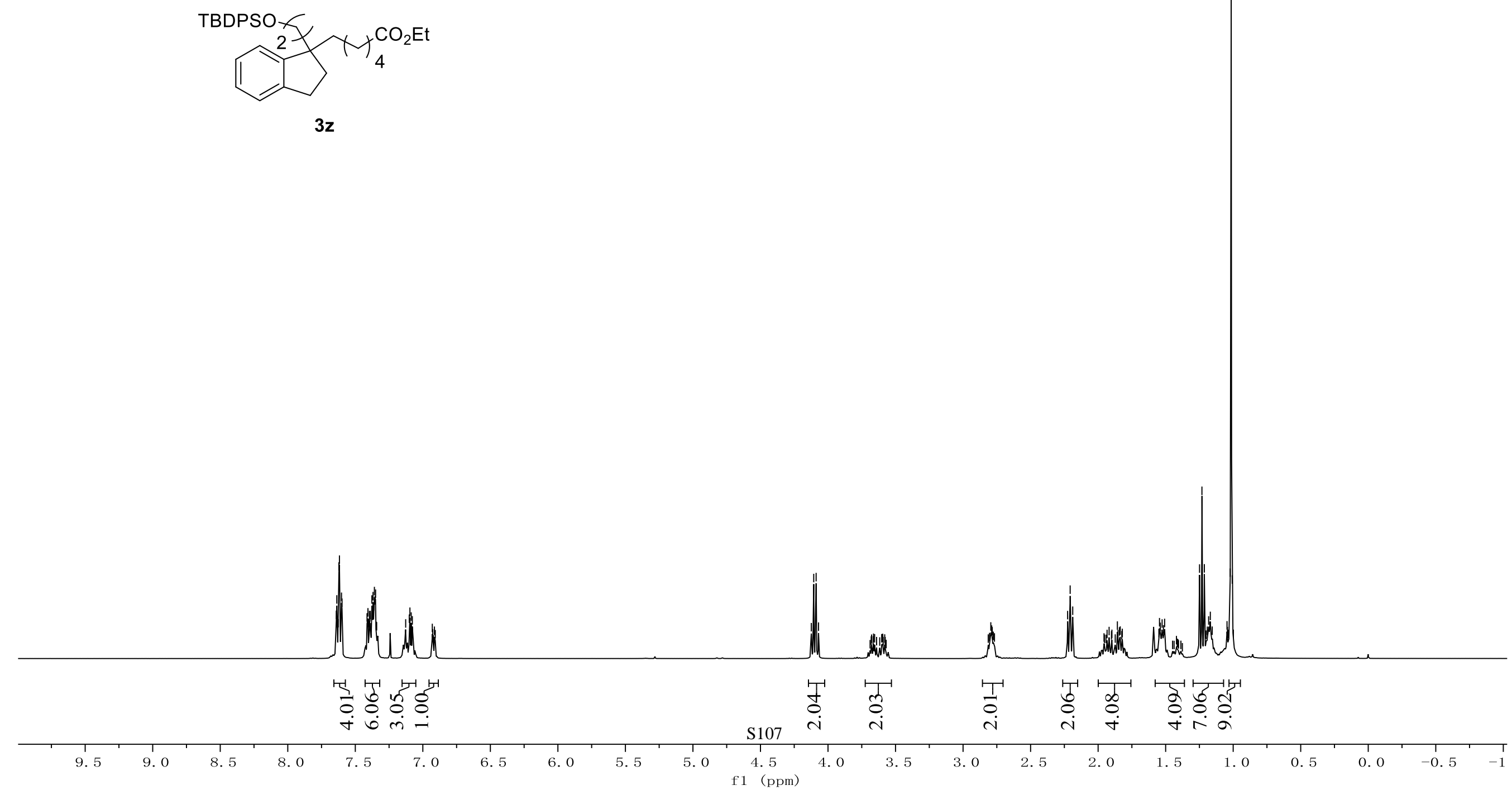

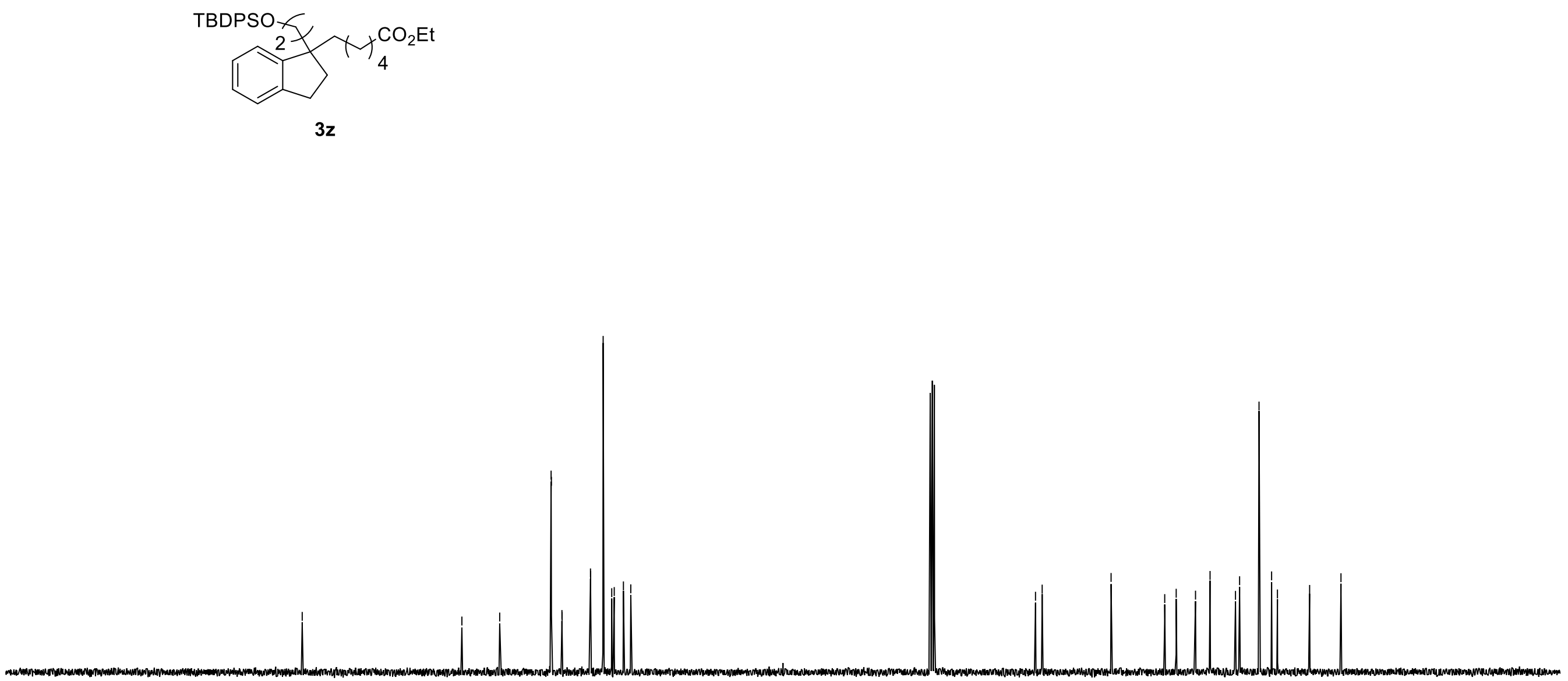

S108

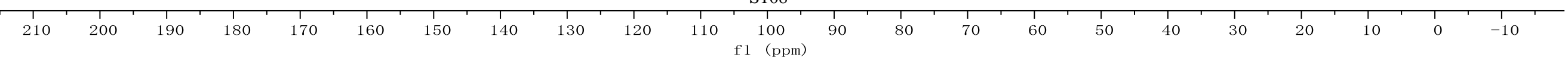




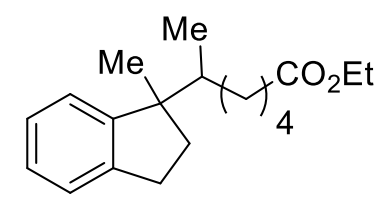

$3 a a$

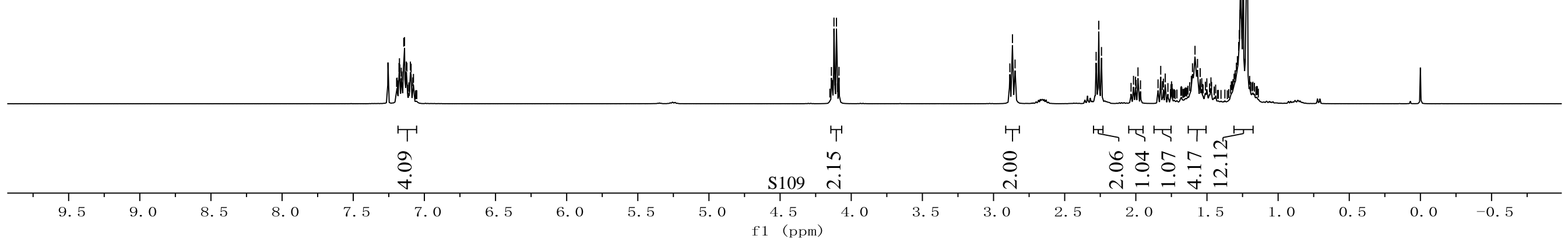




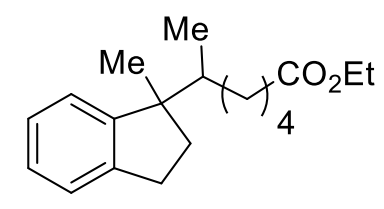

3aa

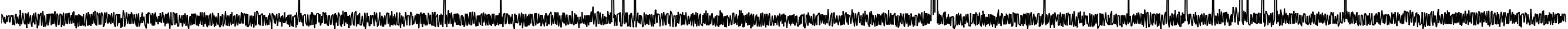




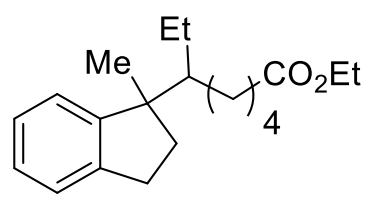

$3 a b$

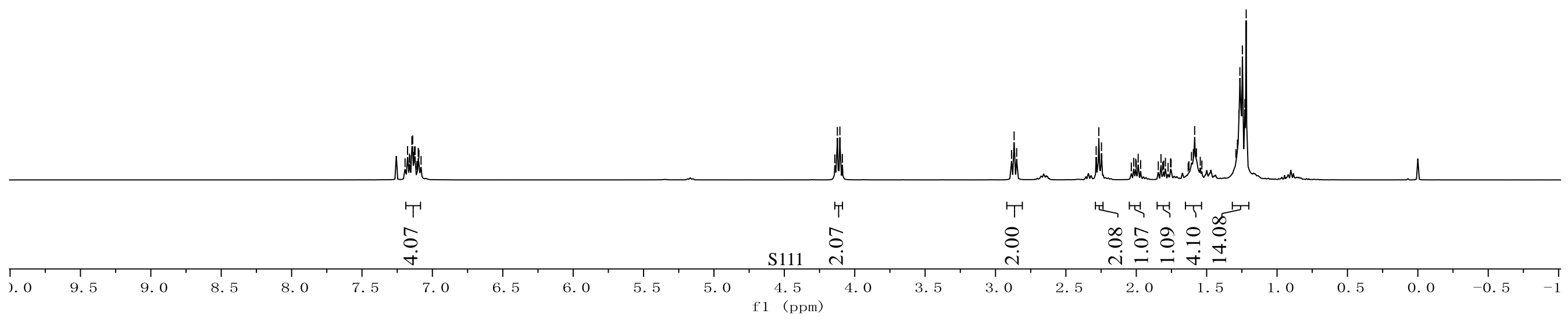




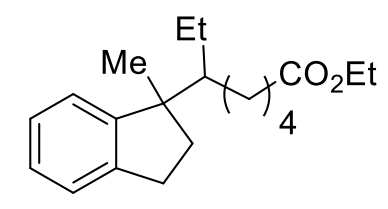

$3 a b$

I

.

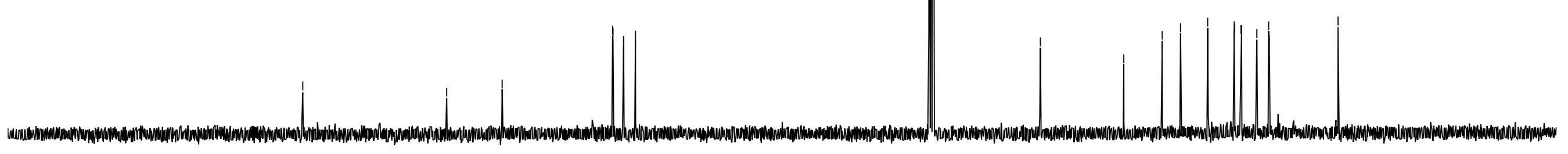
S112

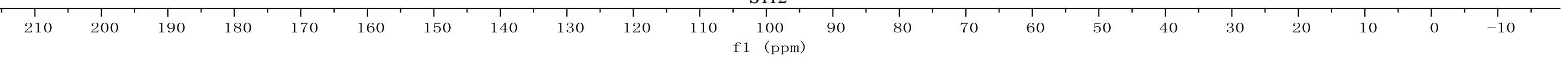




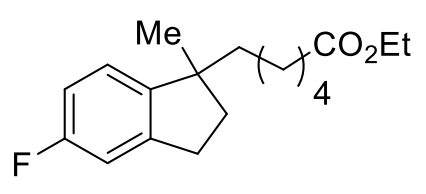

3ac

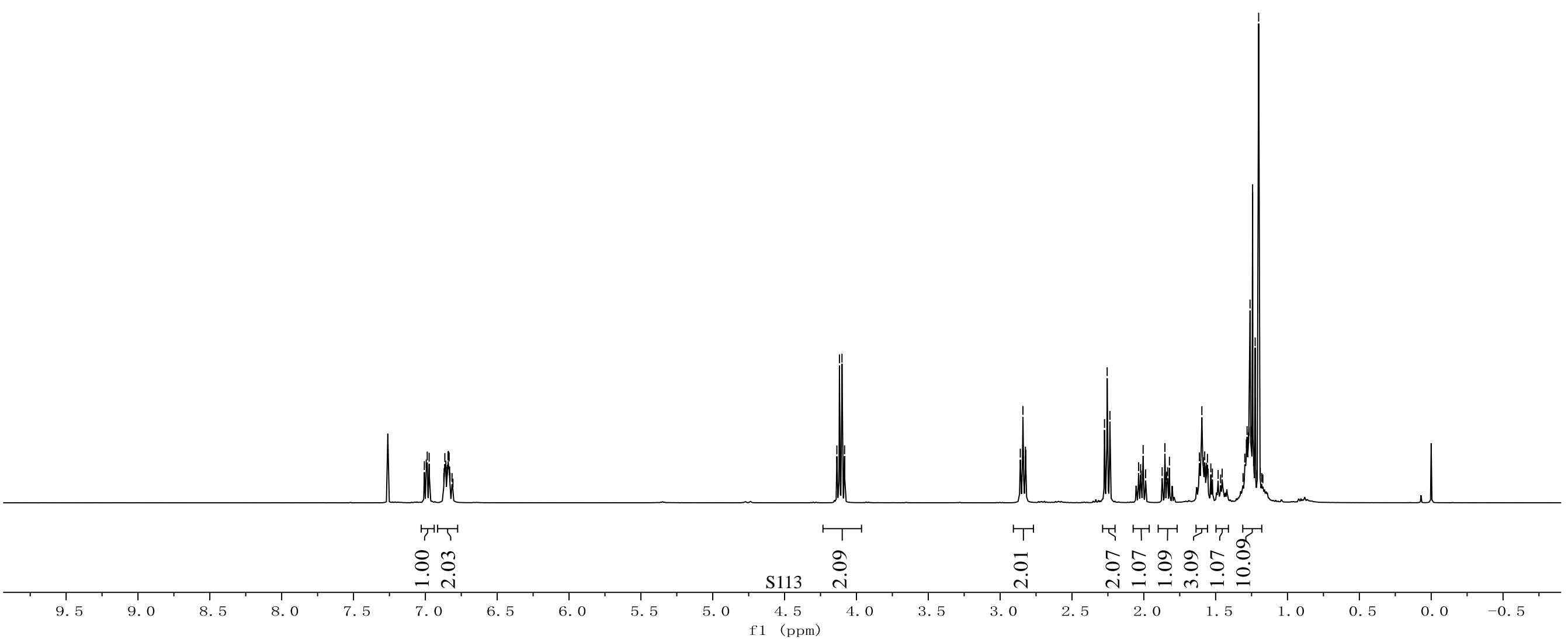




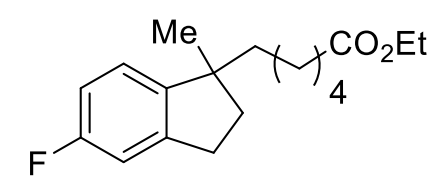

$3 a c$ 


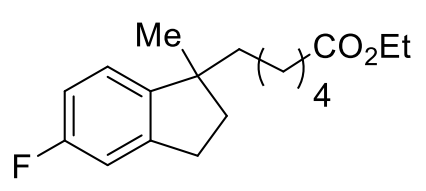

$3 a c$

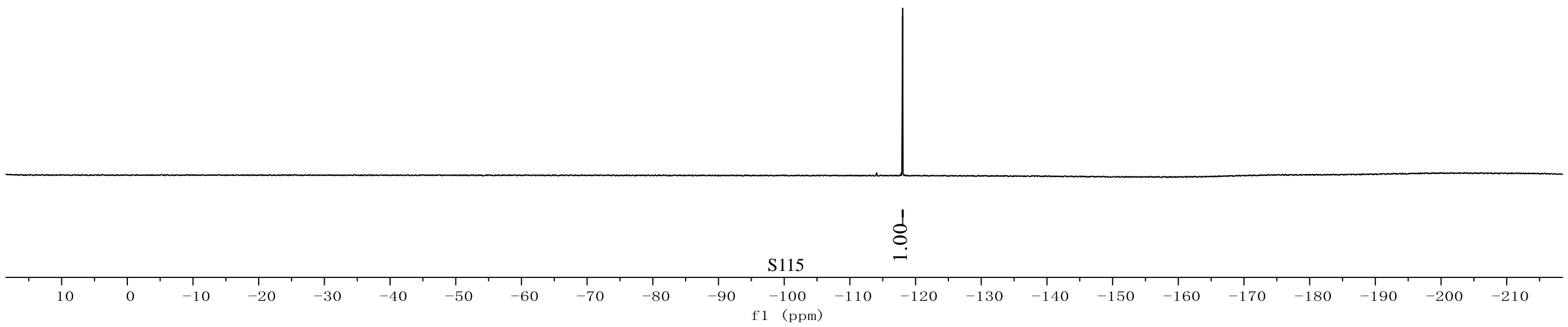




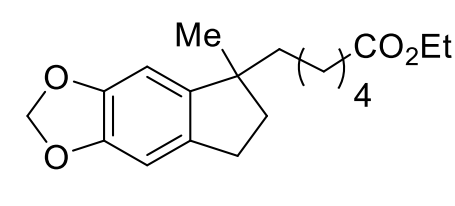

3ad

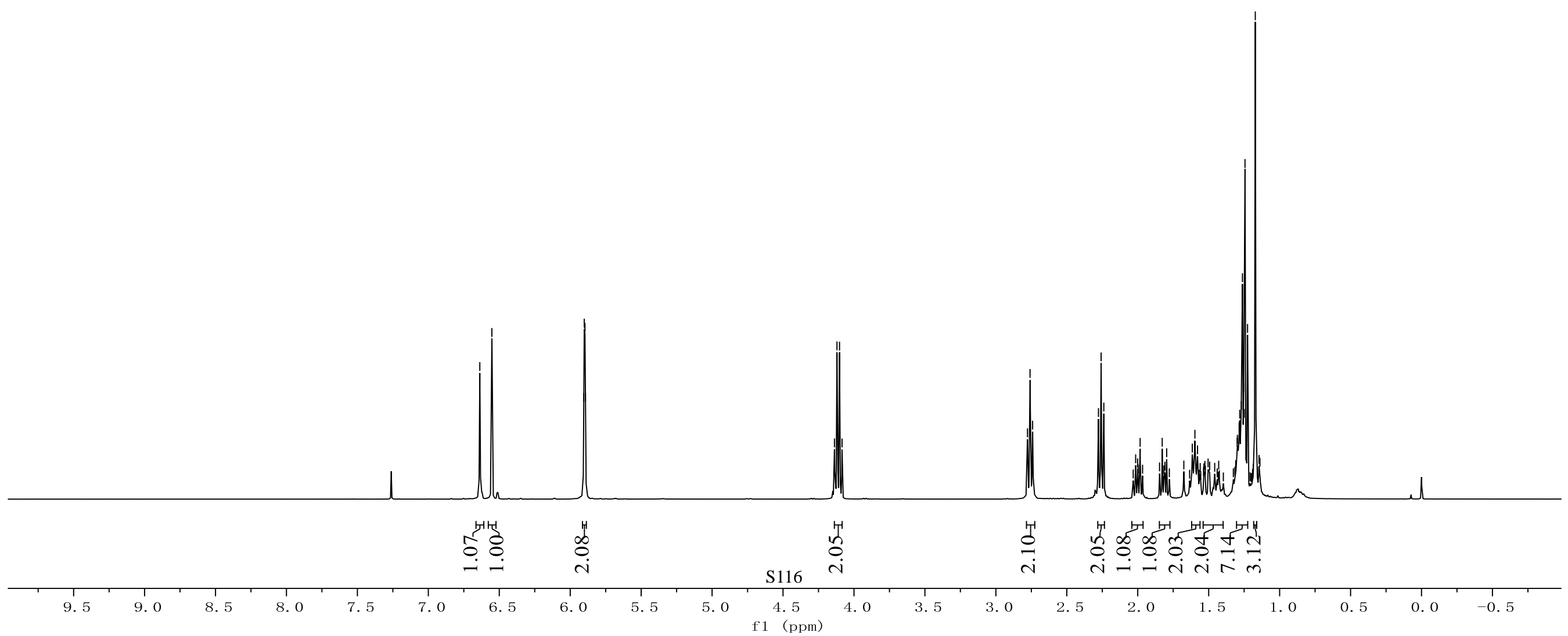




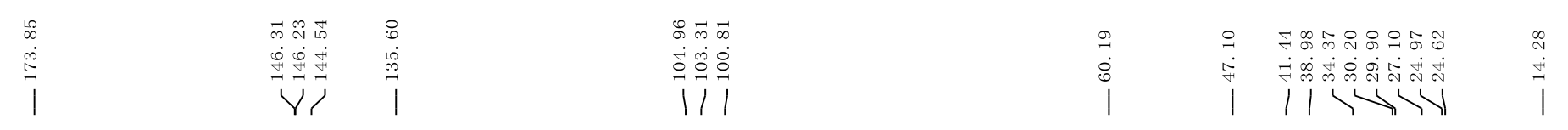
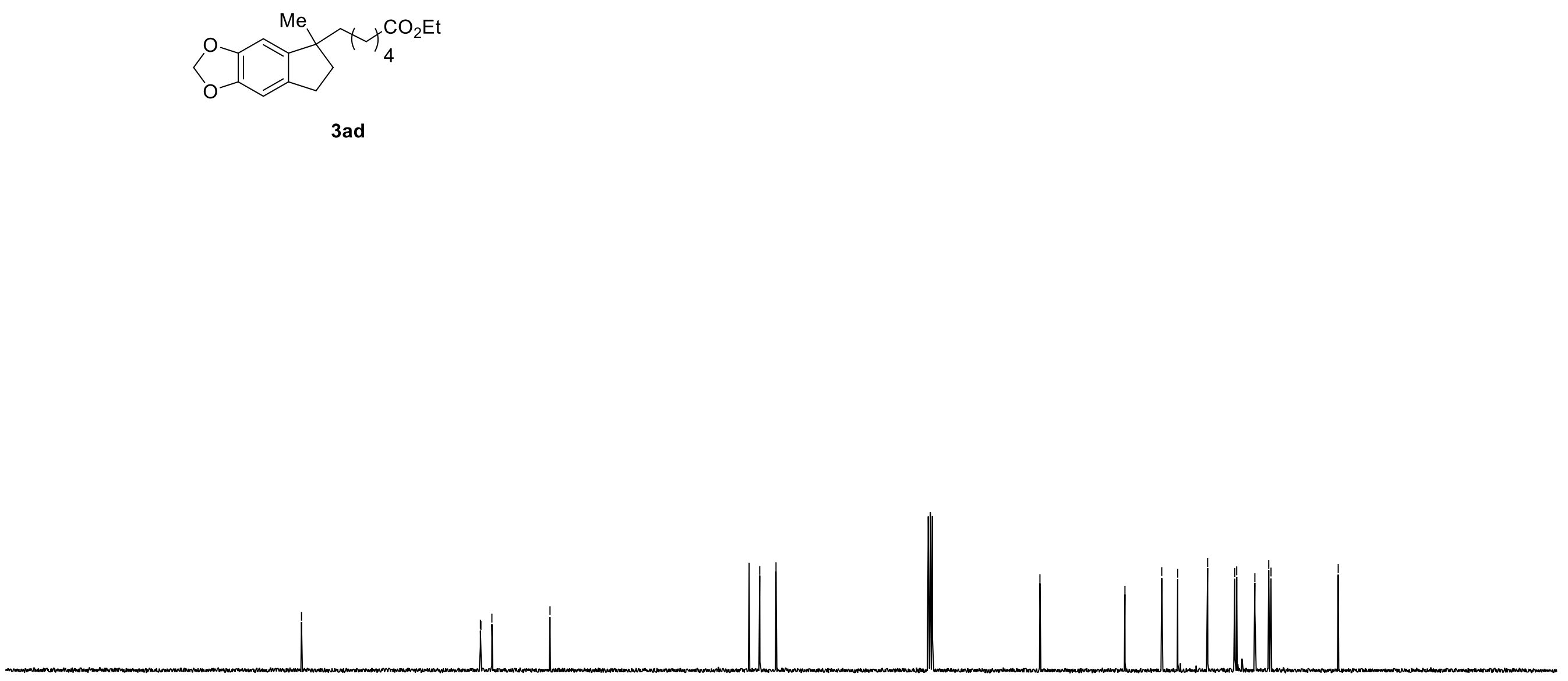

S117

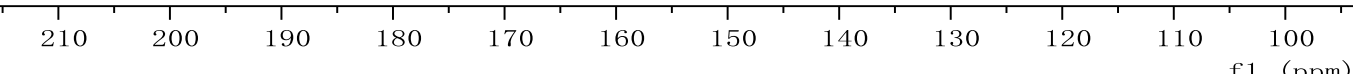

1 (ppm) 


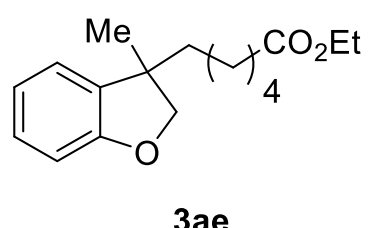

$3 a e$

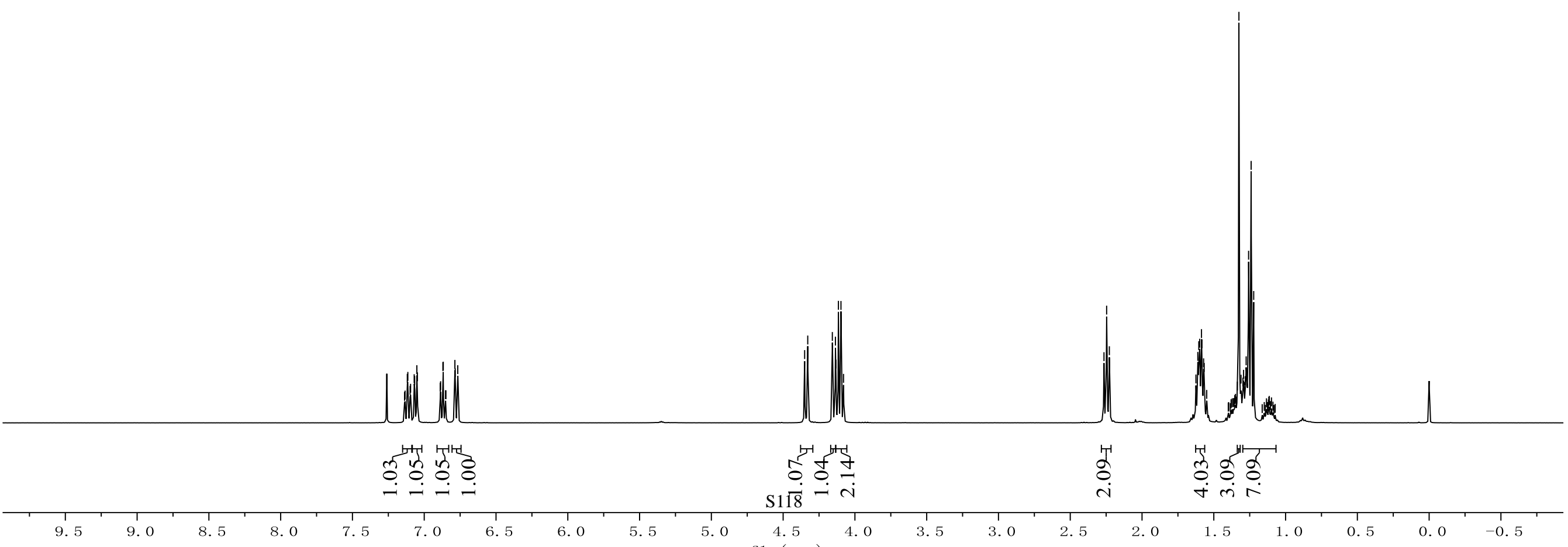




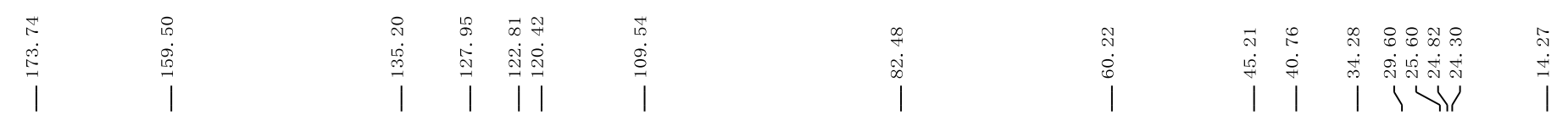

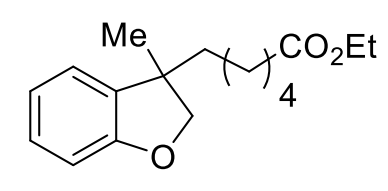

3ae

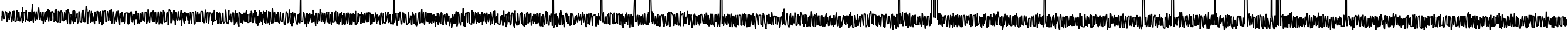

S119

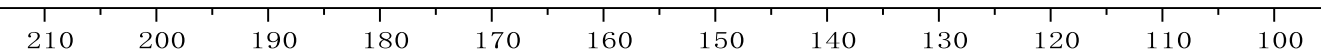

f1 (ppm) 


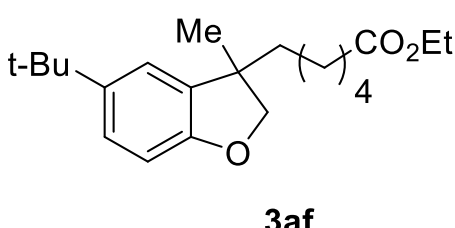

$3 a f$
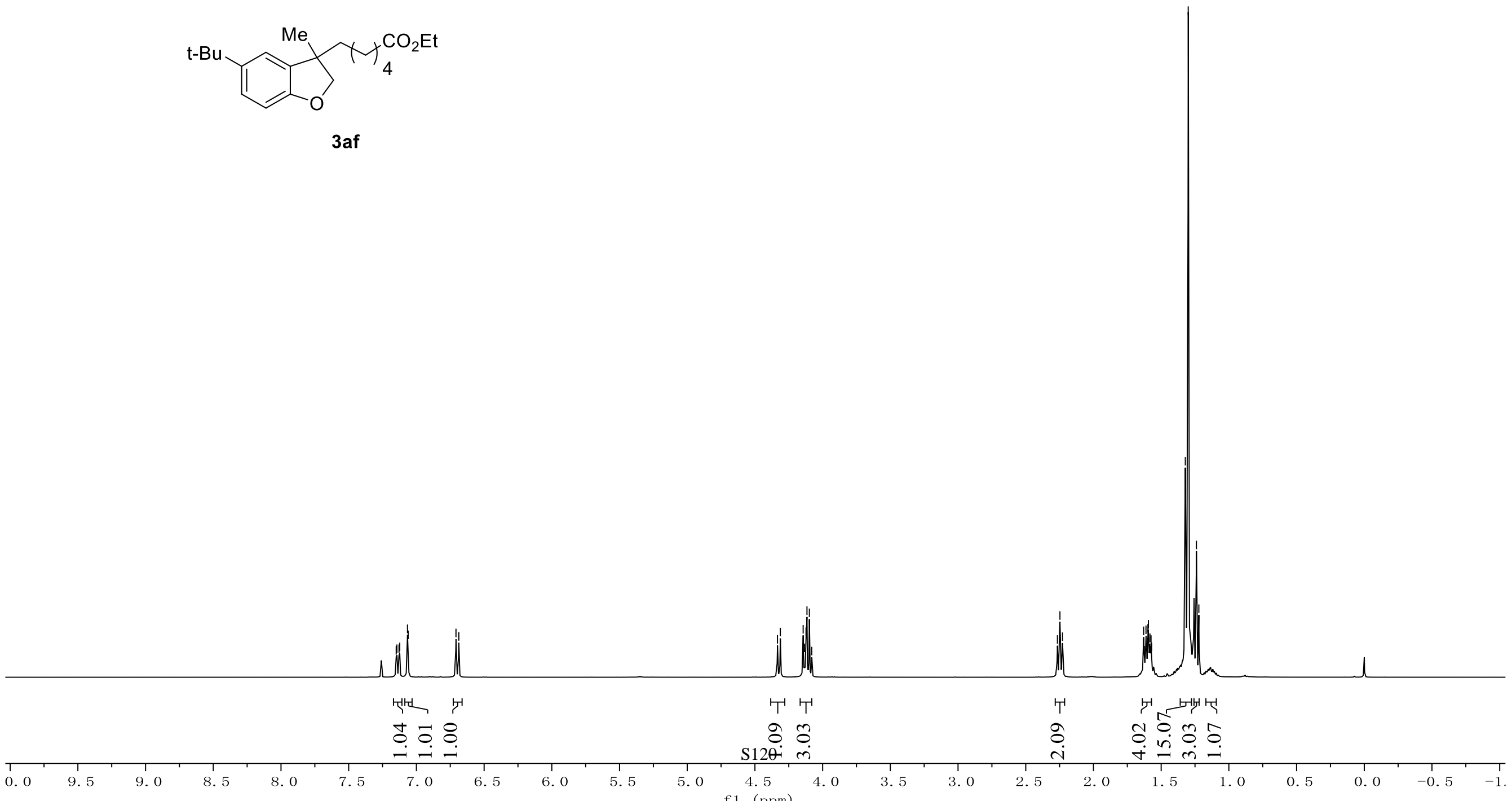


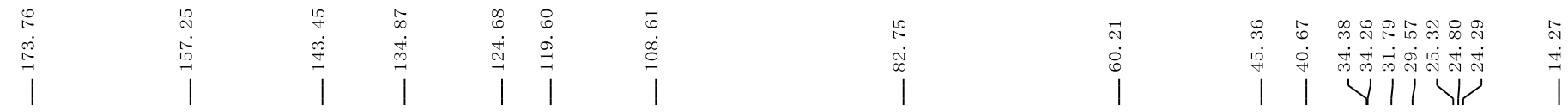

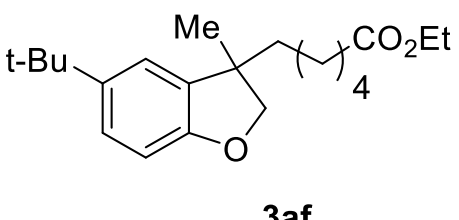

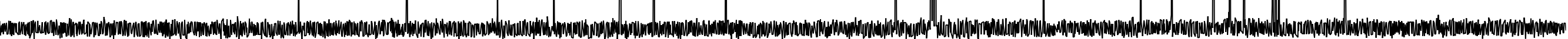

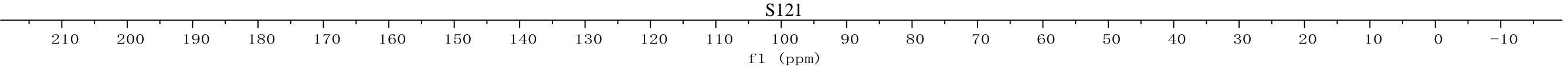


<smiles>CCOC(=O)CCCC1(C)COc2ccc(C#N)cc21</smiles>

3 ag

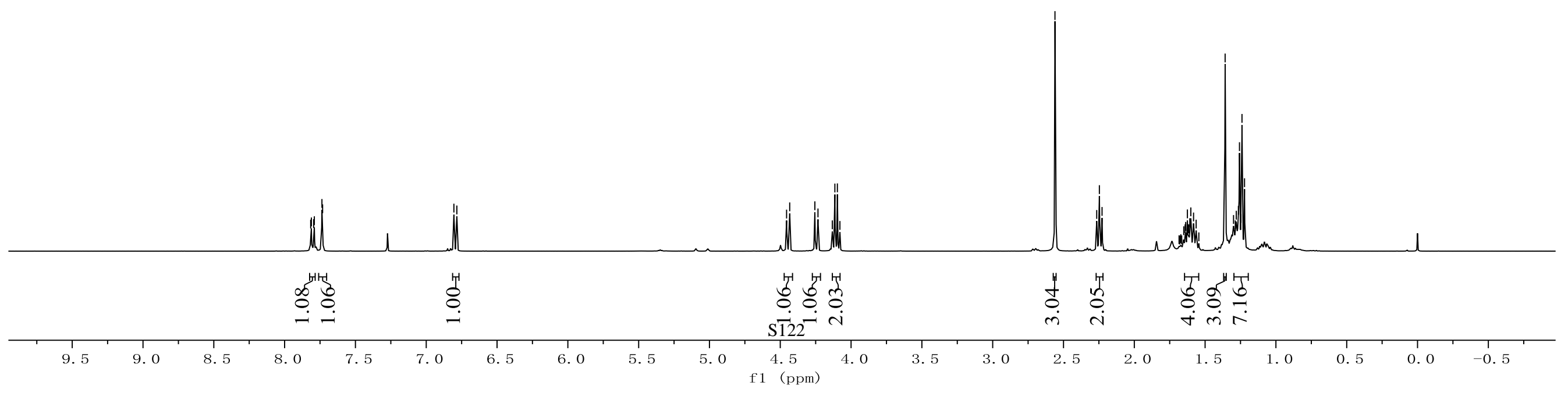




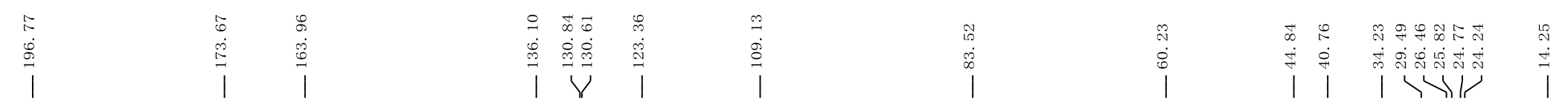
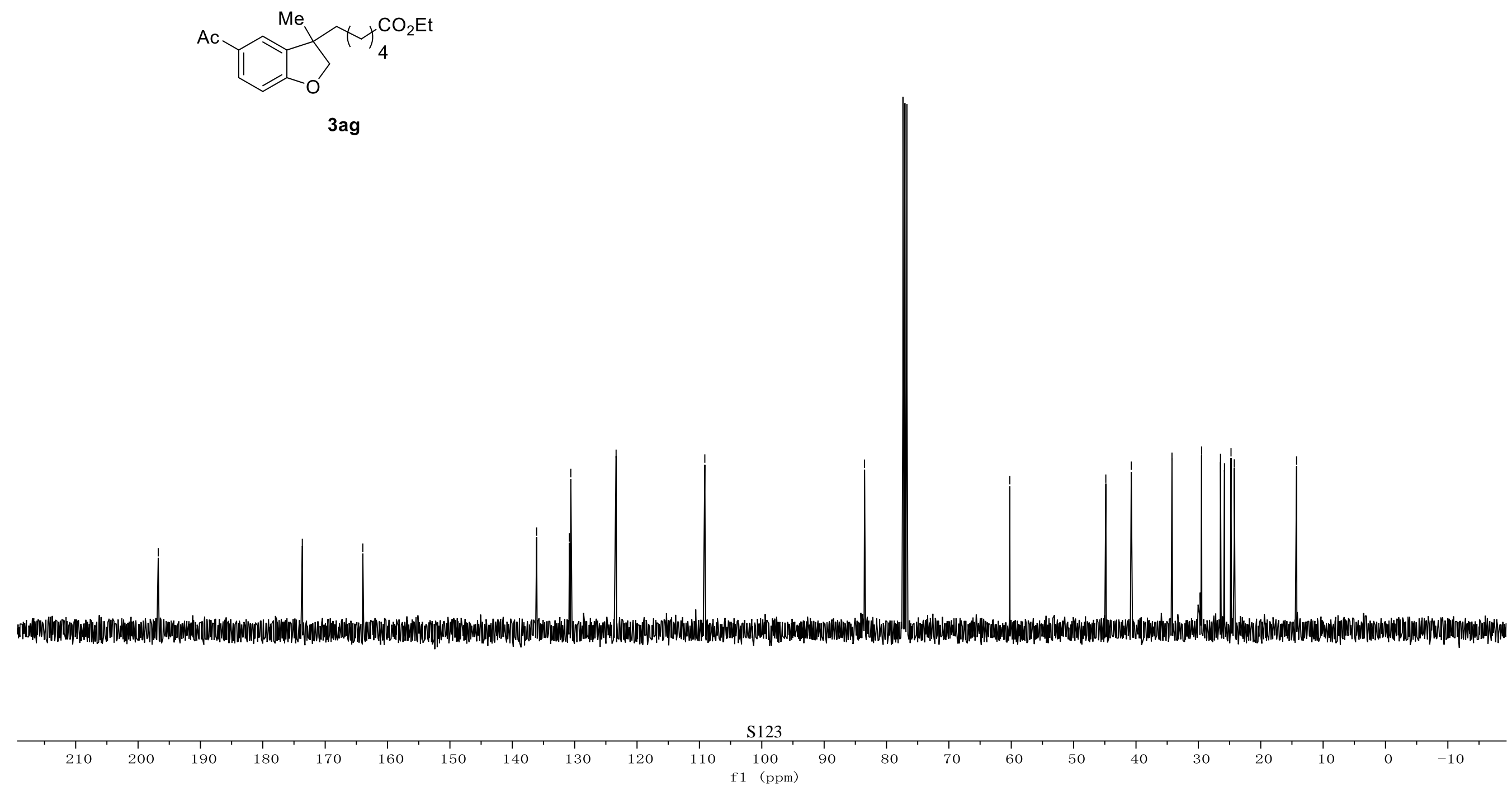


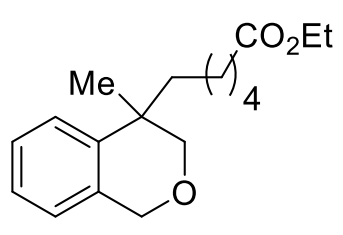

$3 a h$

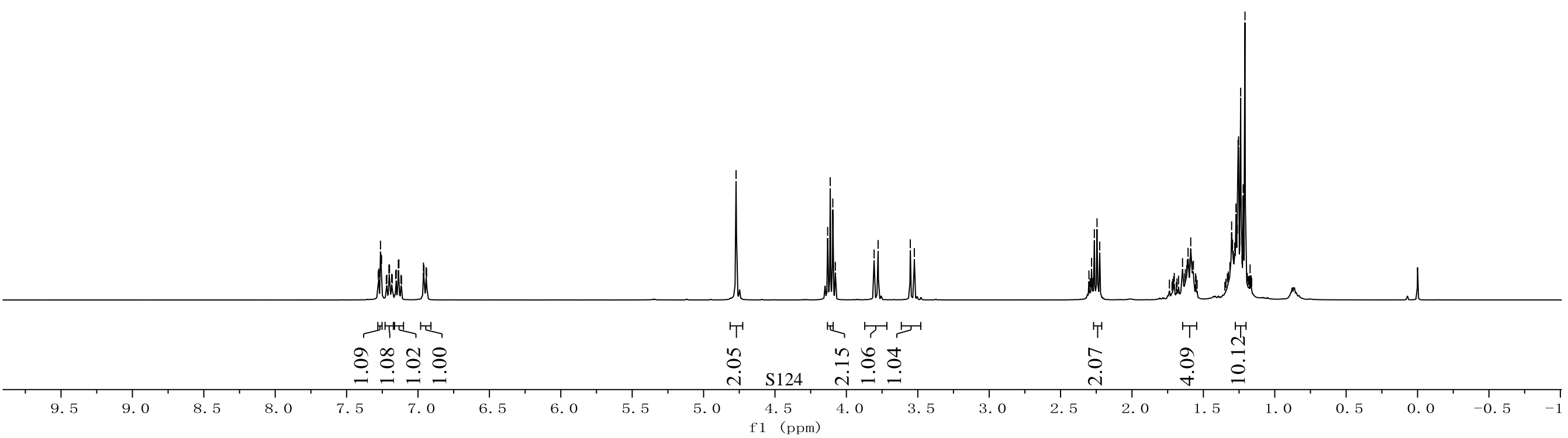




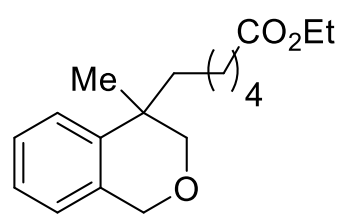

$3 a h$

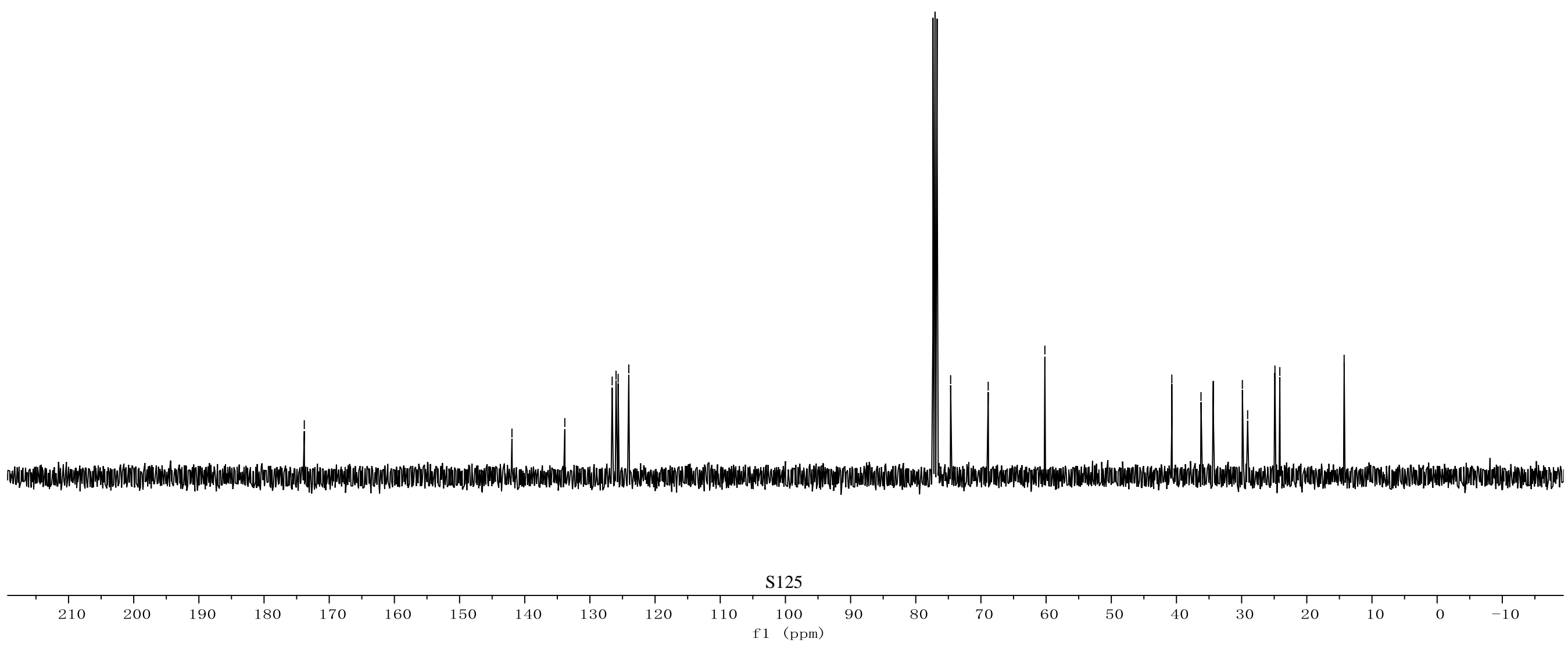



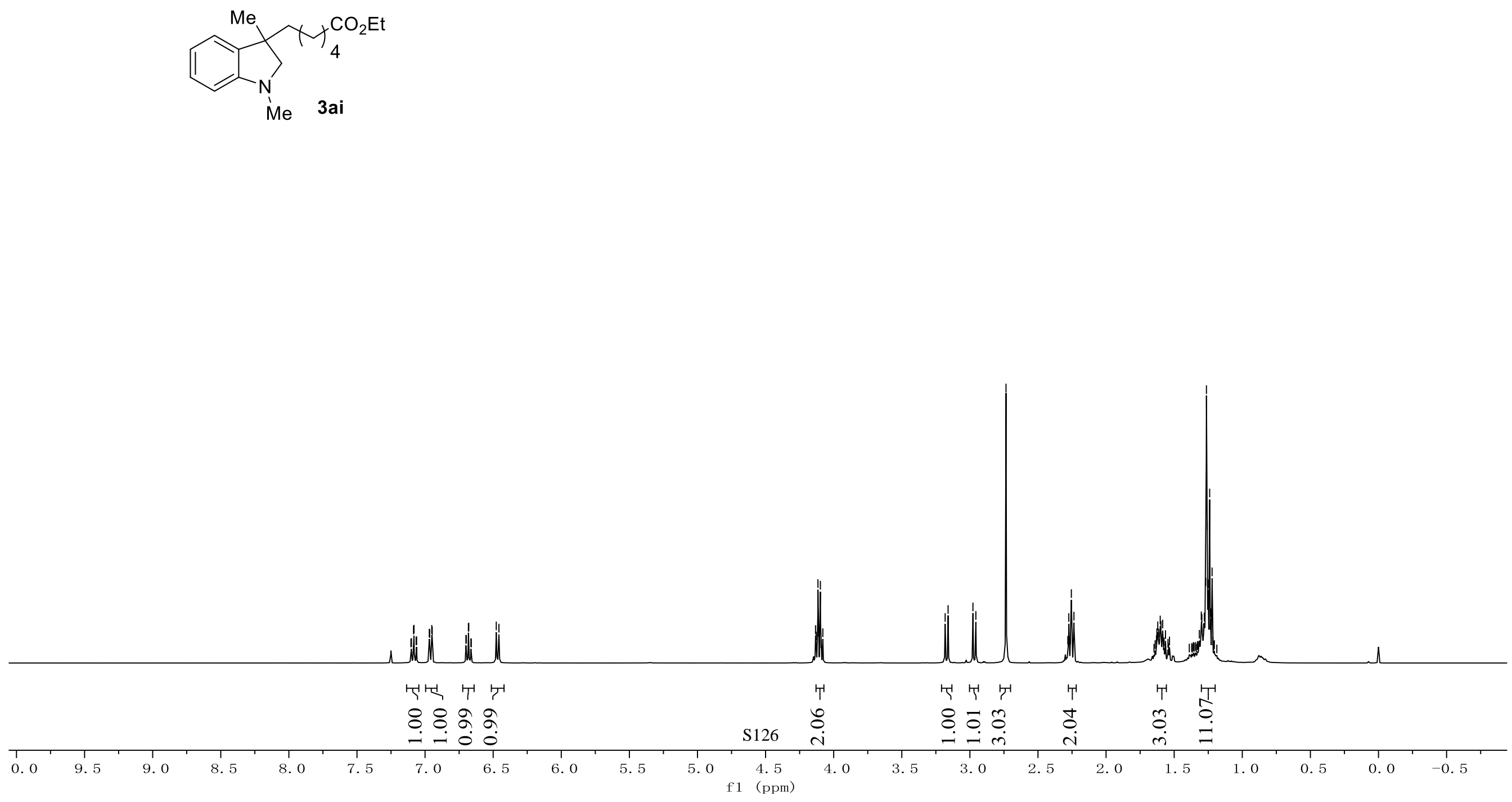


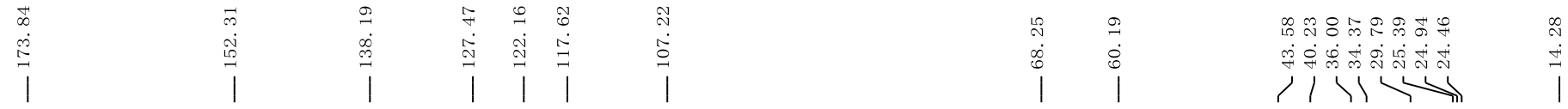
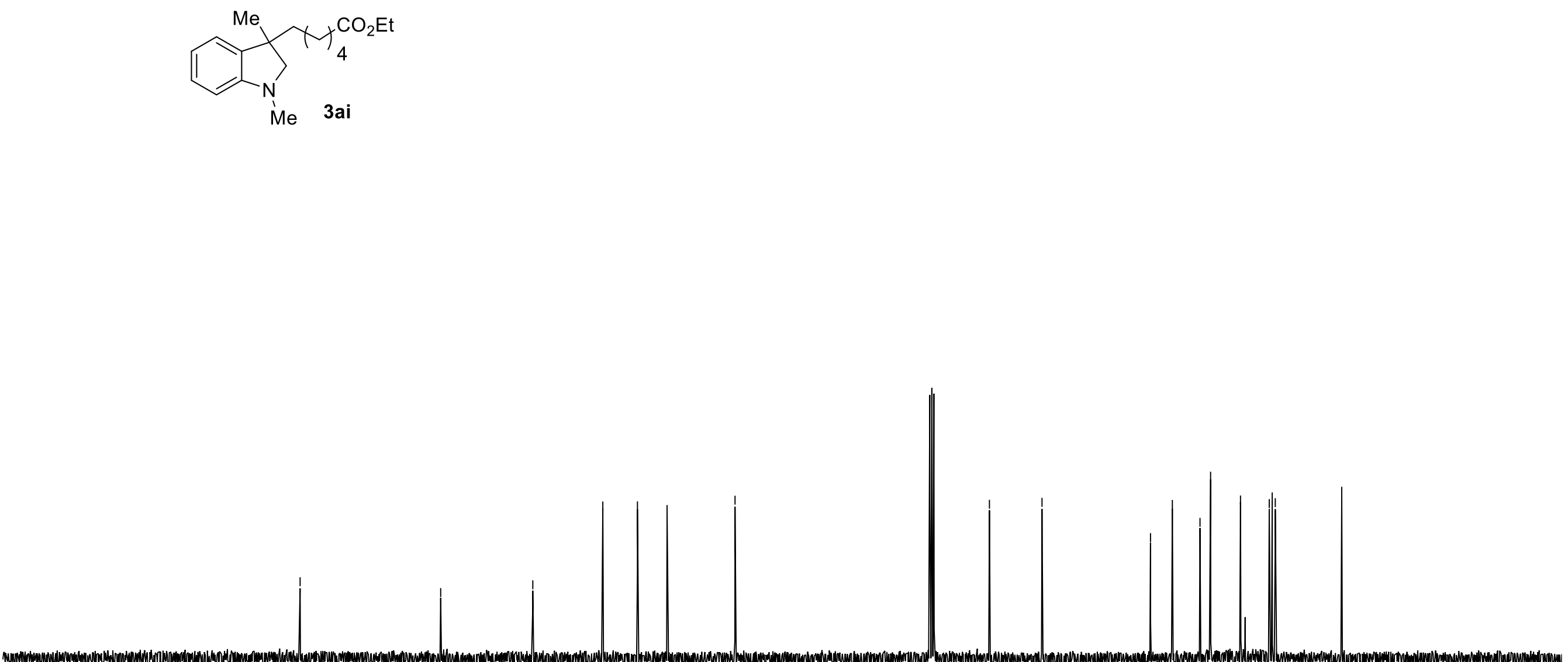

S127

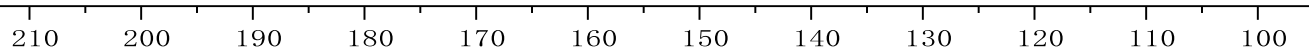

$\mathrm{f} 1 \quad$ (ppm) 

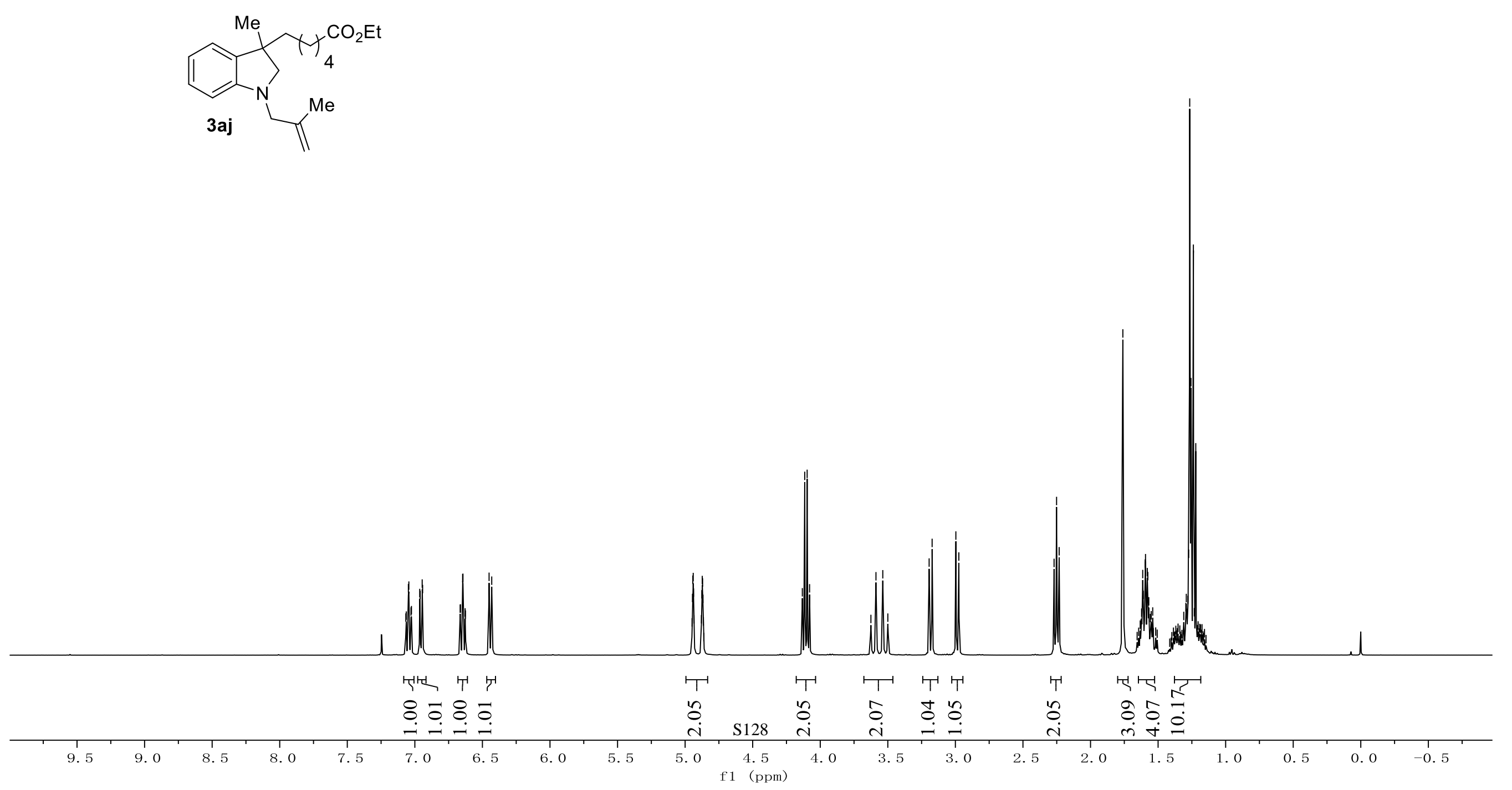


总
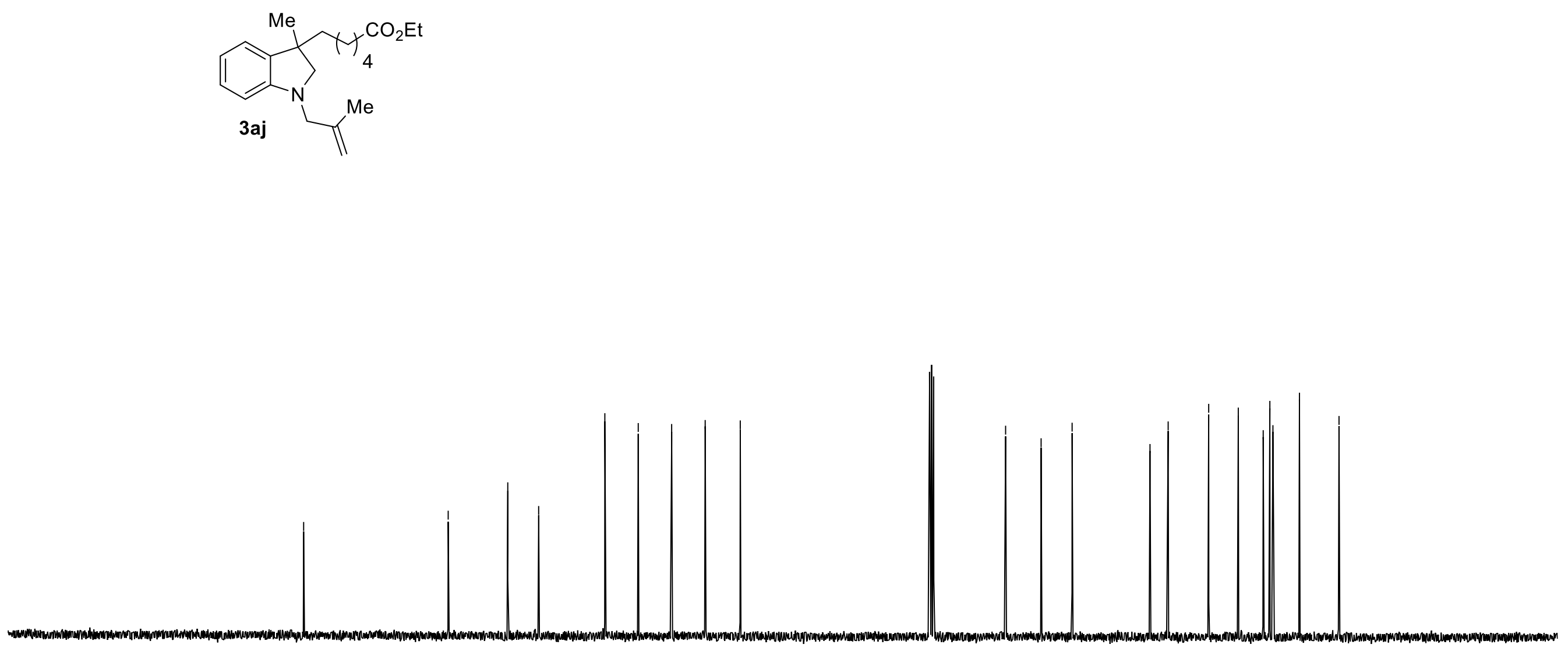

S129

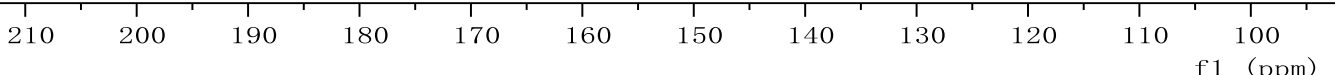




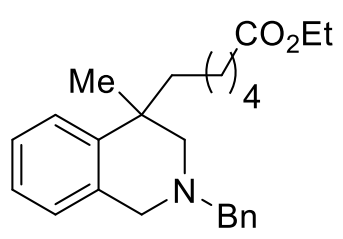

3ak

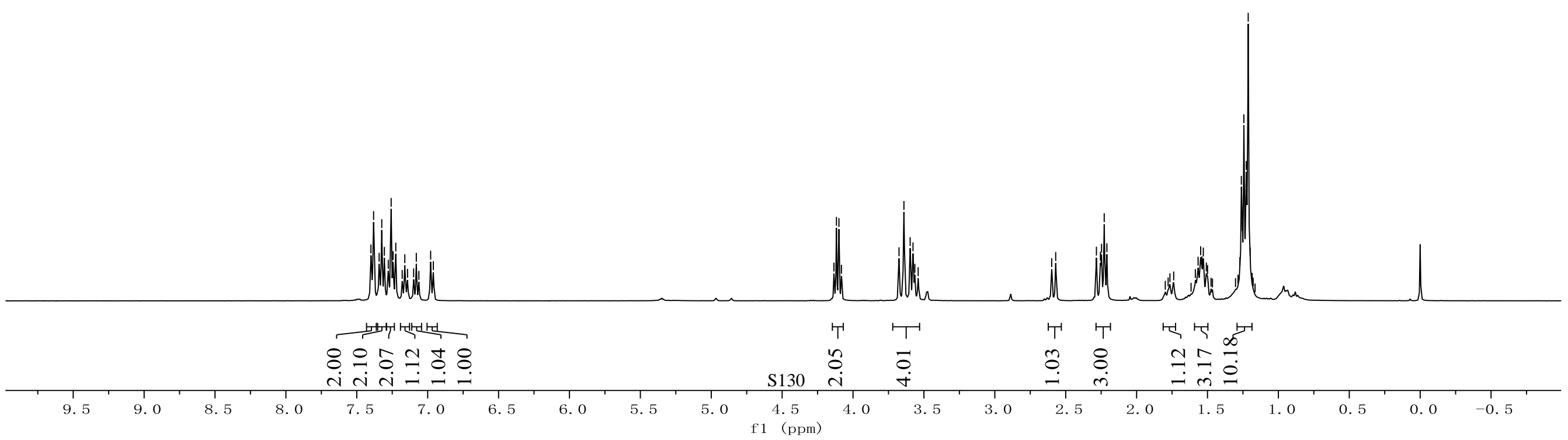




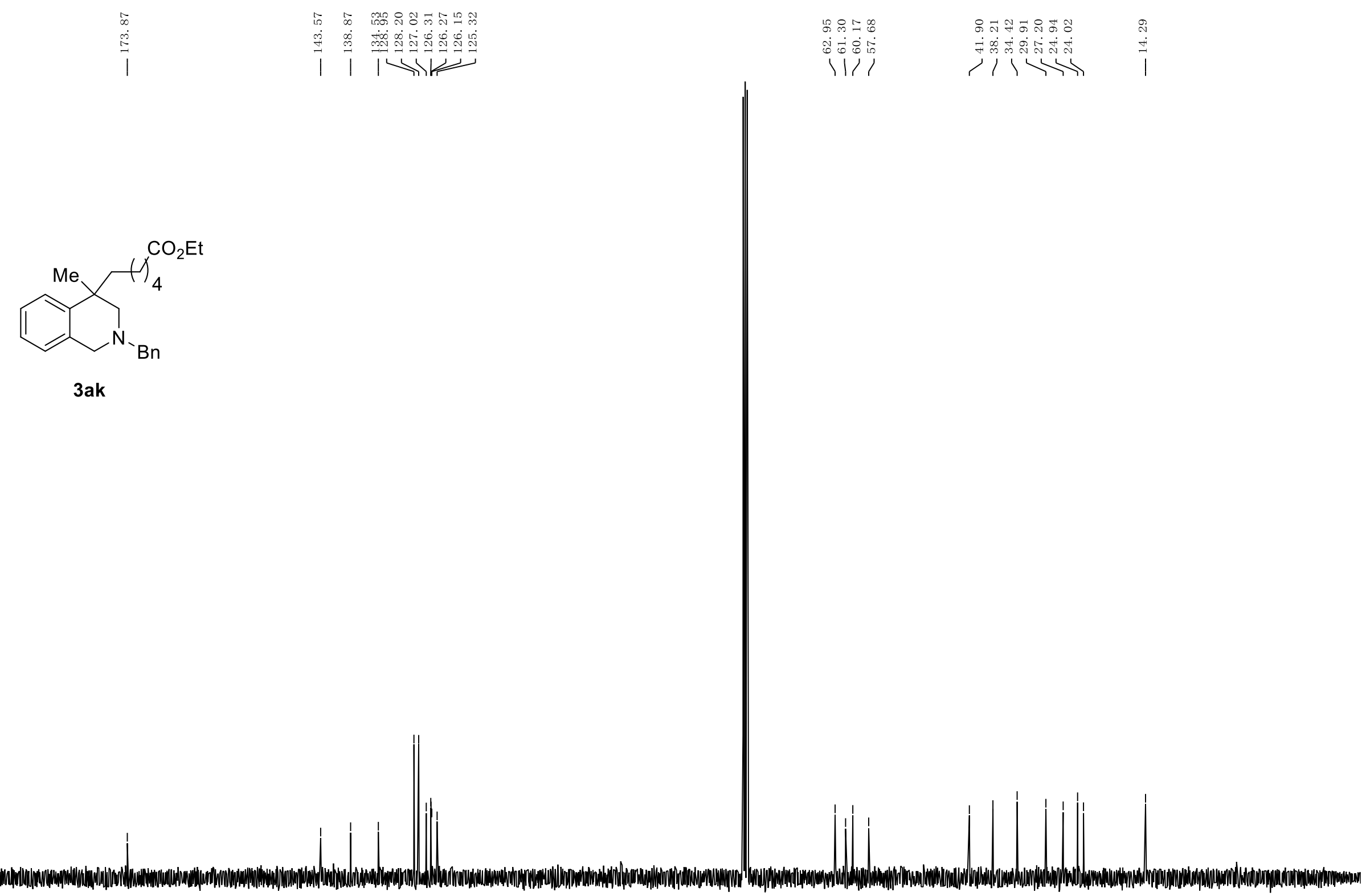

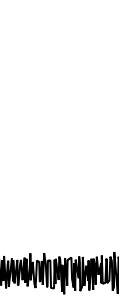

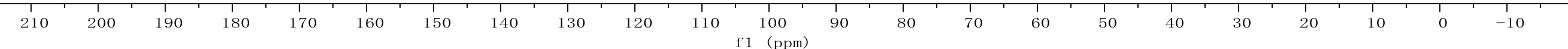




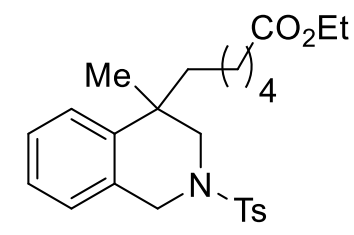

3al

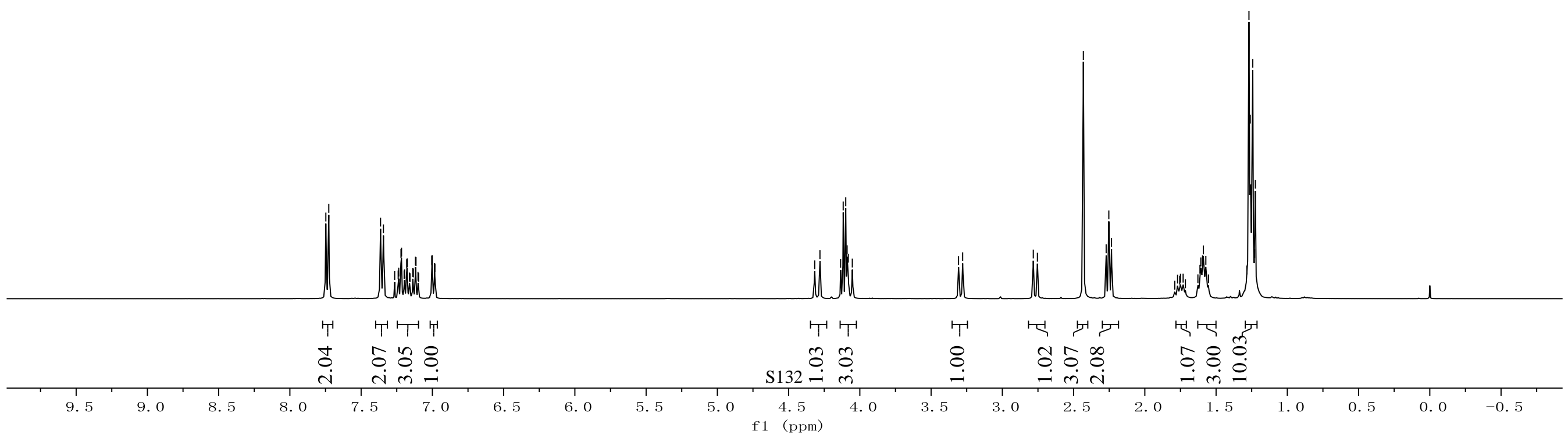




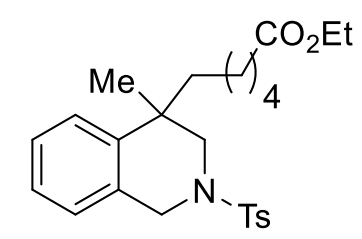

3al
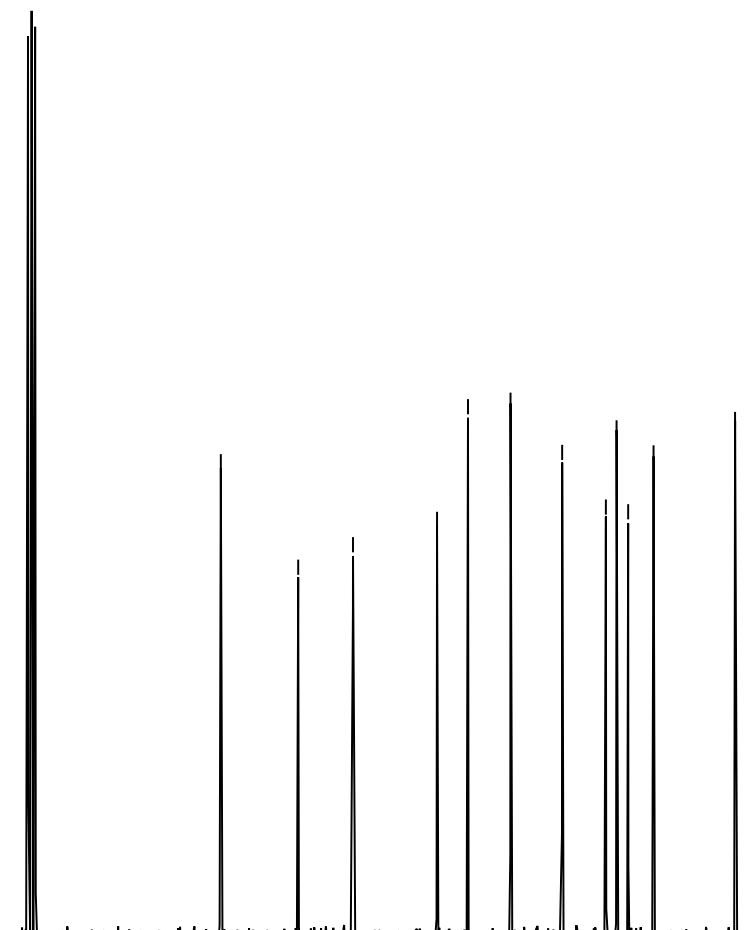

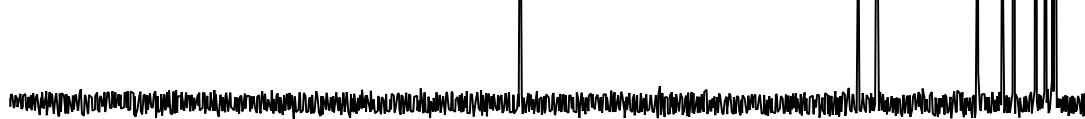

S133

$\begin{array}{lllllllll}1 & 1 & 1 & 1 & 1 & 1 & 1 & 1 & 1\end{array}$

f1 (ppm) 


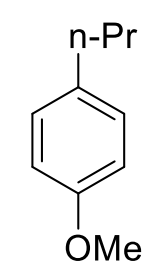

4

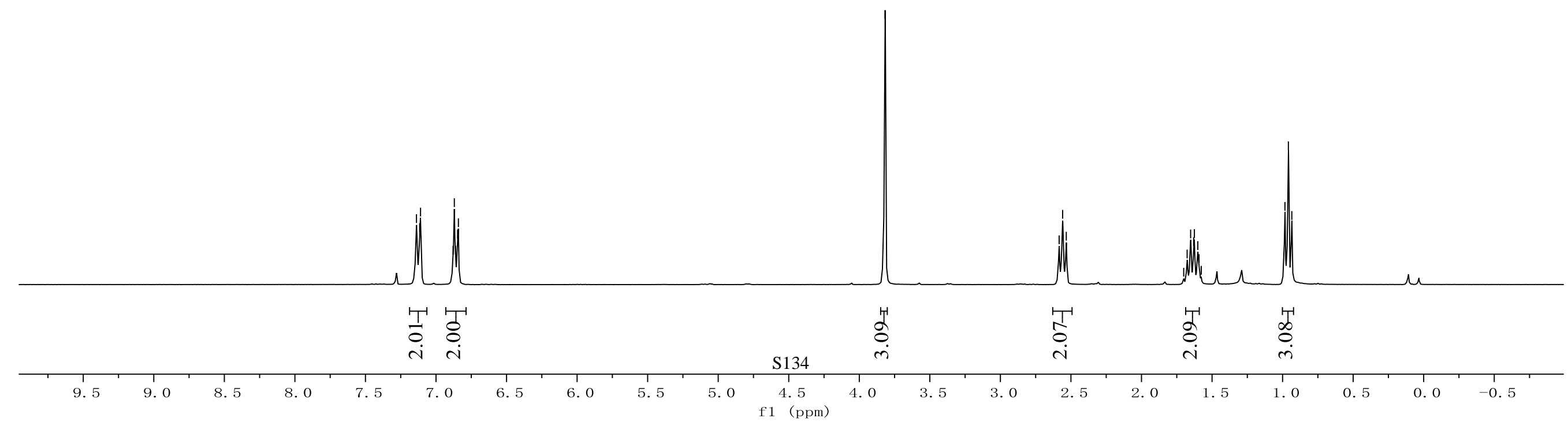




$\begin{array}{lllll}1 & 1 & 1 & 1 & 1\end{array}$
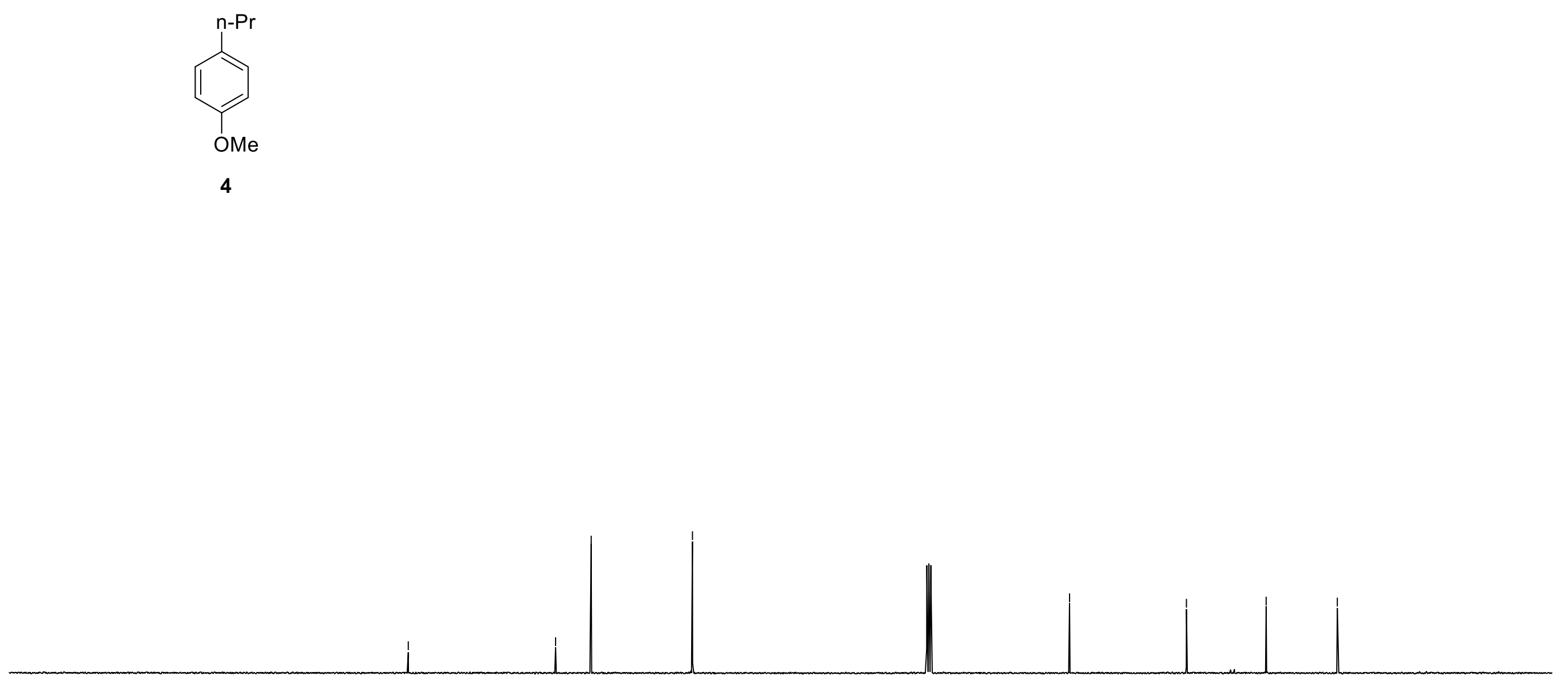

S135

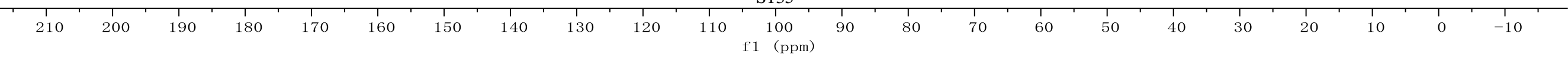



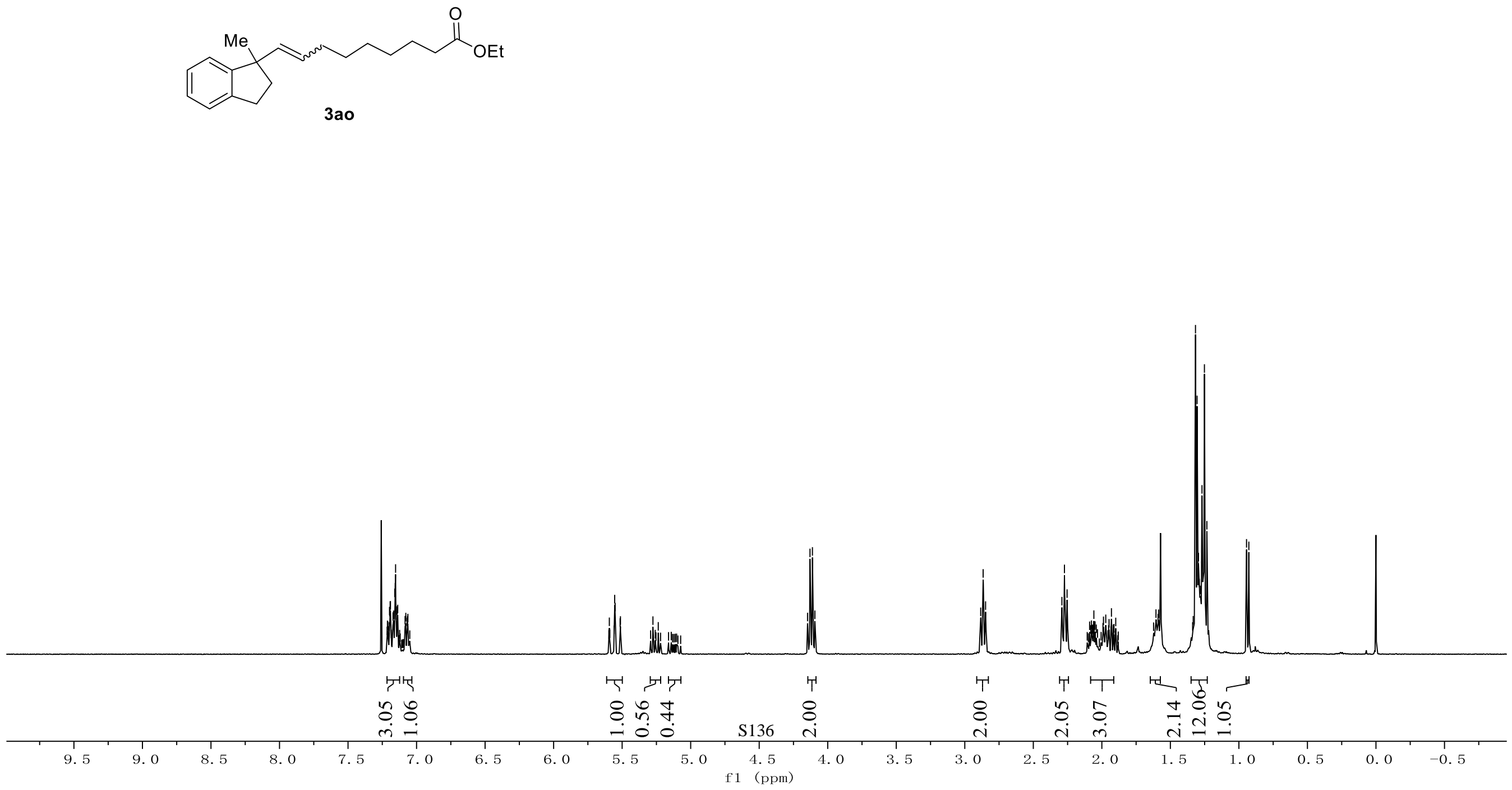


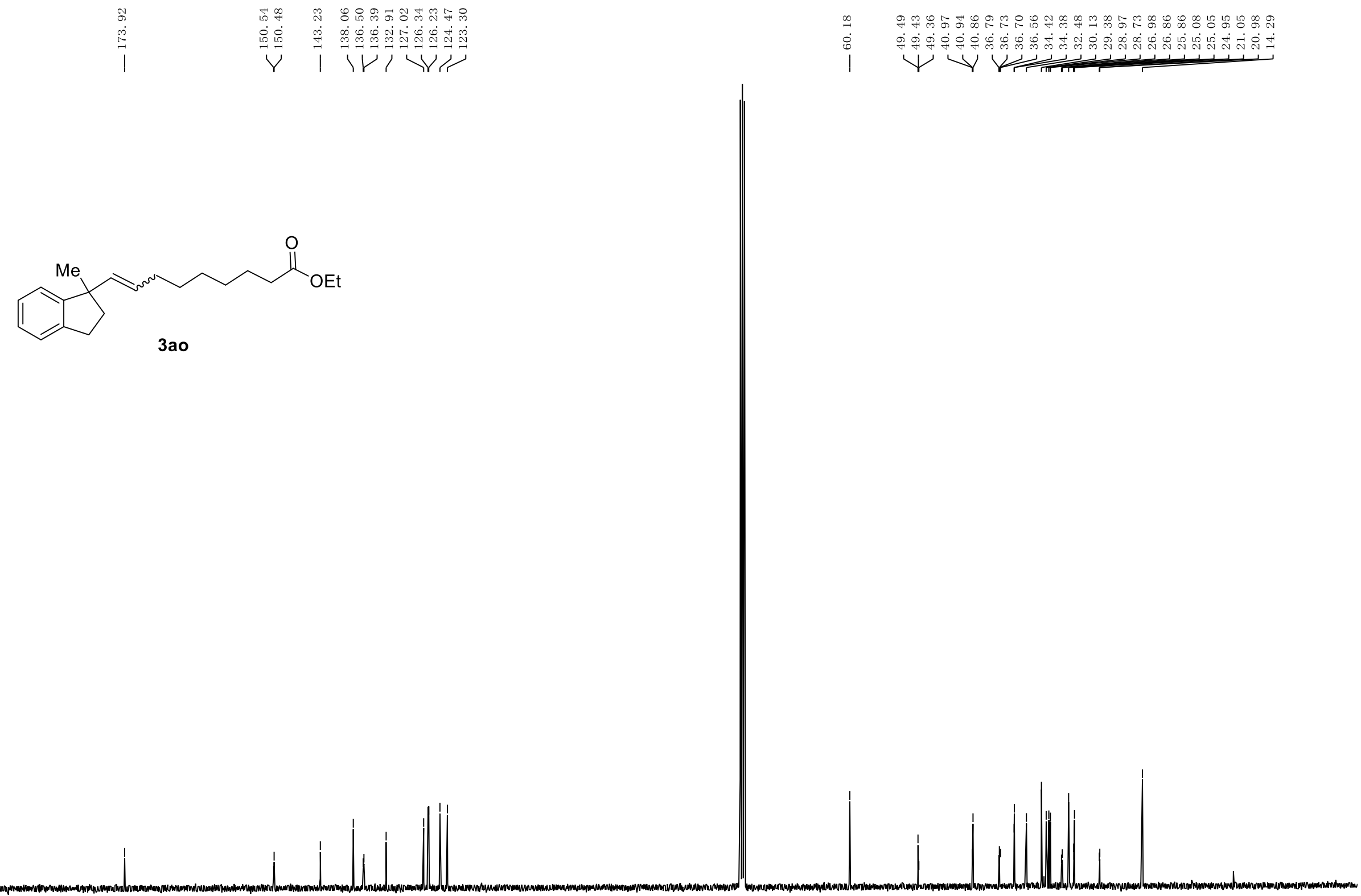

S137

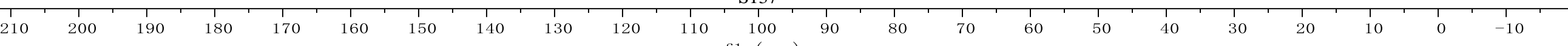

f1 (ppm) 


\section{Copies of HPLC Traces}
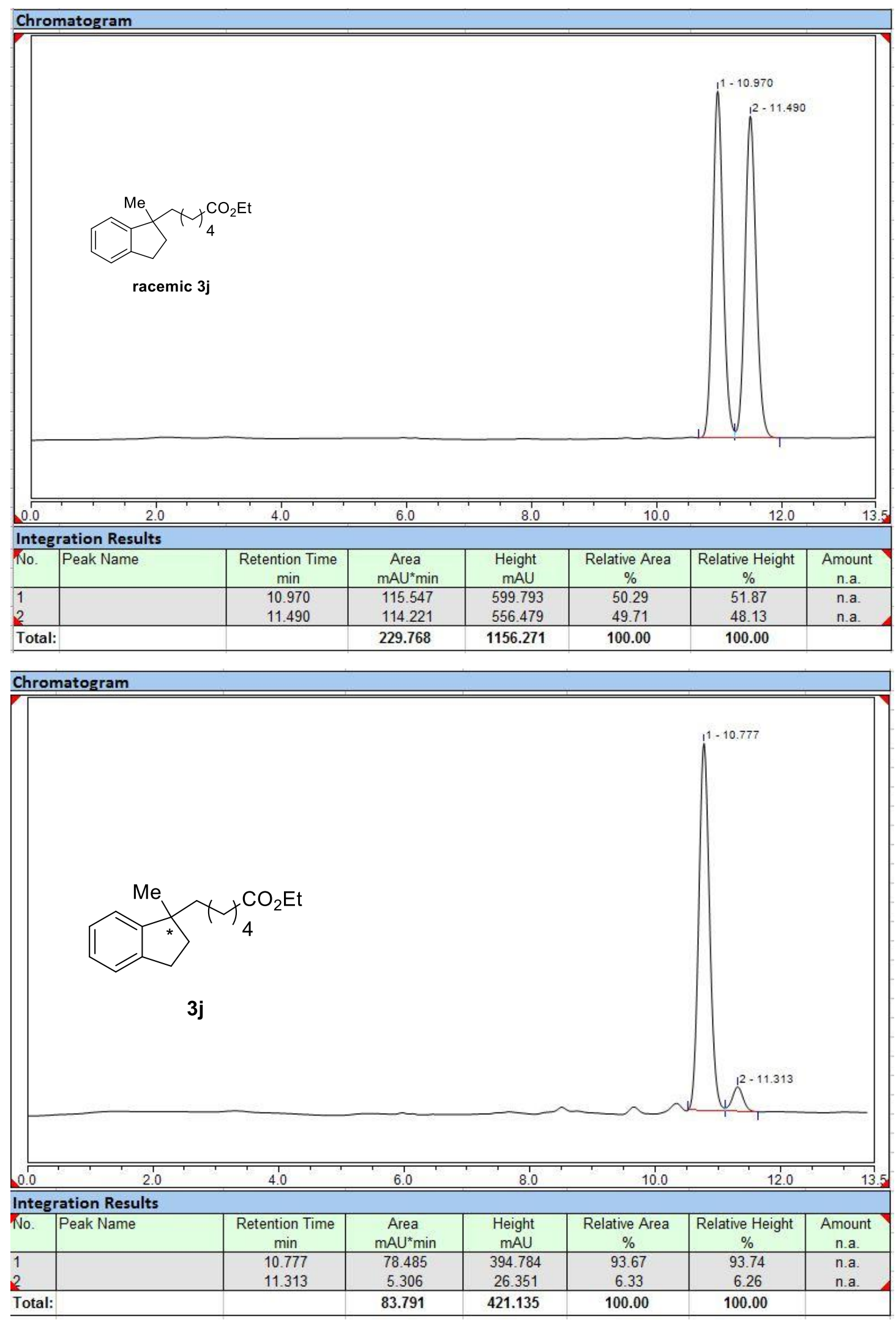

HPLC (Chiralpak OJ-H): $\mathrm{t}_{\mathrm{R}}=10.8$ (major), 11.3 (minor)

Condition: $98: 2 \mathrm{n}-\mathrm{Hexane}: i-\mathrm{PrOH}$, flow rate $0.5 \mathrm{~mL} / \mathrm{min}, 25^{\circ} \mathrm{C}$. 


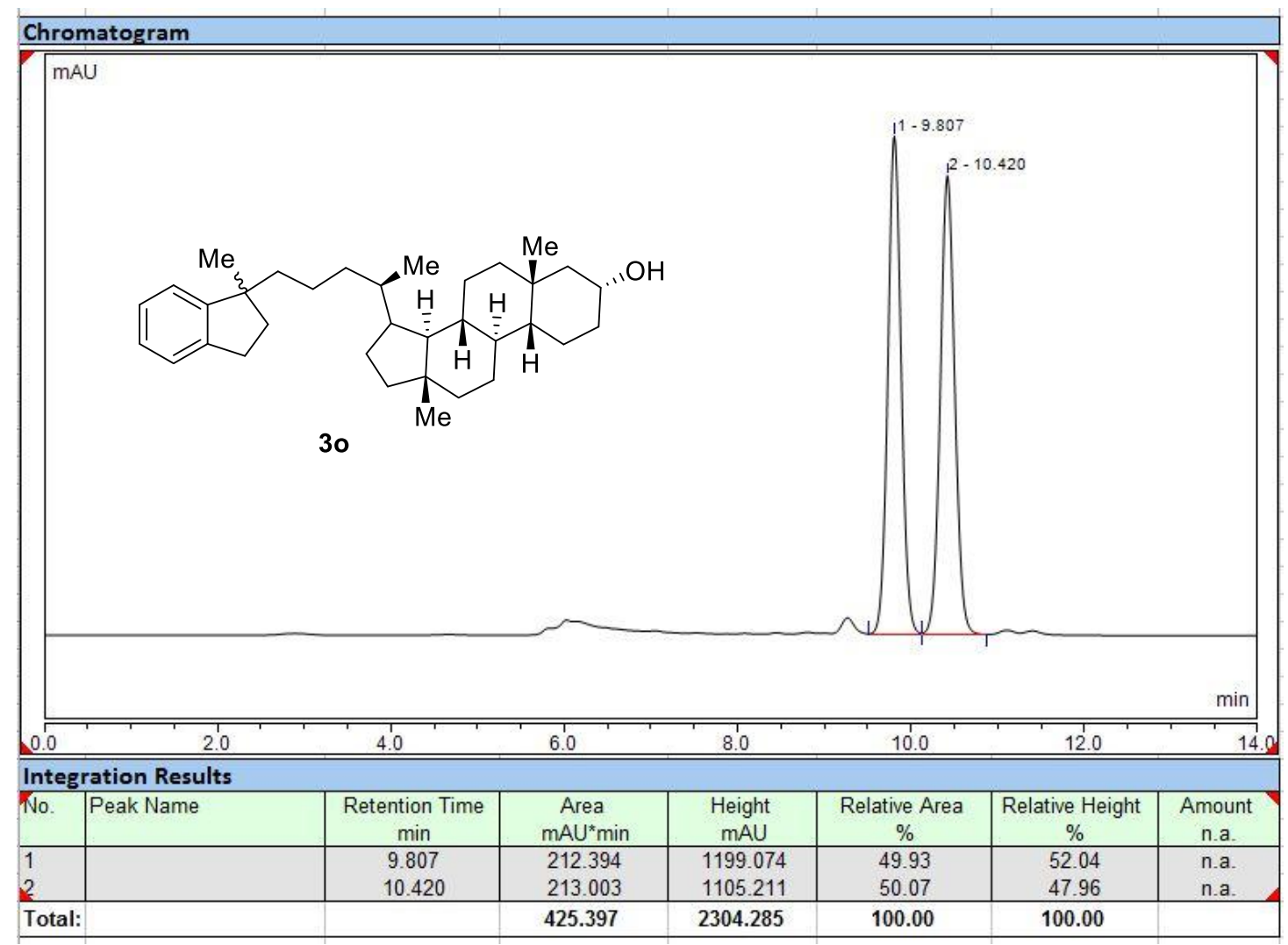

HPLC (Chiral MD): $\mathrm{t}_{\mathrm{R}}=9.8,10.4$

Condition: 80:20 n-Hexane:i-PrOH, flow rate $0.5 \mathrm{~mL} / \mathrm{min}, 25^{\circ} \mathrm{C}$. 